Portland State University

PDXScholar

$1-1-2010$

\title{
Fundamental Studies in Selective Wet Etching and Corrosion Processes for High-Performance Semiconductor Devices
}

Nabil George Mistkawi

Portland State University

Follow this and additional works at: https://pdxscholar.library.pdx.edu/open_access_etds Let us know how access to this document benefits you.

Recommended Citation

Mistkawi, Nabil George, "Fundamental Studies in Selective Wet Etching and Corrosion Processes for HighPerformance Semiconductor Devices" (2010). Dissertations and Theses. Paper 6.

https://doi.org/10.15760/etd.6

This Dissertation is brought to you for free and open access. It has been accepted for inclusion in Dissertations and Theses by an authorized administrator of PDXScholar. Please contact us if we can make this document more accessible: pdxscholar@pdx.edu. 
Fundamental Studies in Selective Wet Etching and Corrosion Processes for High-Performance Semiconductor Devices

by

Nabil George Mistkawi

A dissertation submitted in partial fulfillment of the requirements for the degree of

Doctor of Philosophy

in

Chemistry

Dissertation Committee:

Shankar B. Rananavare, Chair

Margaret Ziomek-Moroz

Makarem A. Hussein

Carl C.Wamser

Albert S. Benight

Andres H La Rosa

Robert Daasch

Portland State University

2010 


\begin{abstract}
As multistep, multilayer processing in semiconductor industry becomes more complex, the role of cleaning solutions and etching chemistries are becoming important in enhancing yield and in reducing defects. This thesis demonstrates successful formulations that exhibit copper and tungsten compatibility, and are capable of Inter Layer Dielectric (ILD) cleaning and selective Ti etching.

The corrosion behavior of electrochemically deposited copper thin films in deareated and non-dearated cleaning solution containing hydrofluoric acid (HF) has been investigated. Potentiodynamic polarization experiments were carried out to determine active, active-passive, passive, and transpassive regions. Corrosion rates were calculated from tafel slopes. ICP-MS and potentiodynamic methods yielded comparable $\mathrm{Cu}$ dissolution rates. Interestingly, the presence of hydrogen peroxide in the cleaning solution led to more than an order of magnitude suppression of copper dissolution rate. We ascribe this phenomenon to the formation of interfacial $\mathrm{CuO}$ which dissolves at slower rate in dilute HF. A kinetic scheme involving cathodic reduction of oxygen and anodic oxidation of $\mathrm{Cu}^{0}$ and $\mathrm{Cu}^{+1}$ is proposed. It was determined that the reaction order kinetics is first order with respect to both $\mathrm{HF}$ and oxygen concentrations.
\end{abstract}

The learnings from copper corrosion studies were leveraged to develop a wet etch/clean formulation for selective titanium etching. The introduction of titanium hard-mask (HM) for dual damascene patterning of copper interconnects created a 
unique application in selective wet etch chemistry. A formulation that addresses the selectivity requirements was not available and was developed during the course of this dissertation. This chemical formulation selectively strips Ti HM film and removes post plasma etch polymer/residue while suppressing the etch rate of tungsten, copper, silicon oxide, silicon carbide, silicon nitride, and carbon doped silicon oxide. Ti etching selectivity exceeding three orders of magnitude was realized. Surprisingly, it exploits the use of $\mathrm{HF}$, a chemical well known for its $\mathrm{SiO}_{2}$ etching ability, along with a silicon precursor to protect $\mathrm{SiO}_{2}$. The ability to selectively etch the Ti HM without impacting key transistor/interconnect components has enabled advanced process technology nodes of today and beyond. This environmentally friendly formulation is now employed in production of advanced high-performance microprocessors and produced in a 3000 gallon reactor. 
In the Loving Memory

$$
\text { of }
$$

My Father, "George Joseph Mistkawi”

\section{This Dissertation}

is Dedicated

to

My inspiring, loving and supportive

Wife Kari, my Son Tristan, my Daughters Serene and Amira, and my Mother Adele 


\section{Acknowledgments}

My years at Portland State will always be cherished, thanks to the many people who have enriched my life, encouraged me, and assisted my efforts throughout this journey, making it all possible and beyond worthwhile. It is difficult to list them all, but I would like to mention some who especially come to mind.

First and foremost, I would like to express my deepest gratitude towards my supervising Professor Shankar Rananavare, for he has been much more than just a great teacher and role model. He has been a great advisor and a true friend to me. Dr. Shankar has taken care of me by kindly giving me the opportunity to work with him since my first days at Portland State, from providing research guidance and looking out for my professional career to offering a caring ear when I needed it. His technical prowess, professionalism, and personal integrity remain as qualities I will keep striving to emulate. It has truly been a pleasure to be a part of his research family and I look forward to continue what I hope is just the beginning.

I'm grateful to Dr. Margaret Ziomek-Moroz of the Department of Energy Research Center (Albany, Oregon) for being such a wonderful mentor and a friend. It is a blessing to have a mentor who is an excellent listener and constant source of ideas and technical support. My involvement with Dr. Margaret was unmistakably the catalyst in my progress. I thank her for her kindness and continuous friendly support and advice.

I am also indebted to Dr. Makarem Hussein of Intel and humbly thank him for his perpetual trust in me for opening up the door of my cherished dream to pursue my 
Ph.D. Dr. Makarem has been actively involved in advising me too, without his support and collaboration with Dr. Rananavare and Dr. Ziomek-Moroz, it would have been much more difficult to reach this achievement. I thank him for his wonderful kindness and friendship.

I would like to acknowledge and thank Intel Corporation for the financial support received throughout the years.

Then, I would like to give thanks and appreciation to Professor Albert S. Benight, Professor Carl C. Wamser, Professor Andres H La Rosa, and Professor Robert Daasch for their kindness to spare their invaluable time to serve in my dissertation committee. I extend the same gratitude to Vani Thirumala, Dr. Hok-Kin Choi (Intel Corporation, Fab Materials Operations Labs, Santa Clara, CA.), Fuji Film Electronic Materials Labs, and Dr. Lourdes Dominguez (Intel Corporation, Logic Technology Development) for their help and support.

Many thanks go to my research colleagues and friends; Dr. Joo Chan, Dr. Allen Chaparadza, Hong Tran, and all the members of the Rananavare Research Group, for their intellectual help and support all through these years. I'm also grateful to my colleagues and friends Mr. Bruce Beattie, Dr. Erica Thompson, Mr. Gary Martin, and Dr. Lana Jong of Intel for their genuine kindness and support.

I also humbly remember and thank my colleagues and friends from the undergraduate years who are still close friends, Dr. Paul Sunderland, Mr. Richard Morgan, and Mr. Brian Turner for their help and support and for their friendship. 
Finally, I want to talk about and thank my family members without whom, this would have been impossible. I am deeply indebted to my family for their relentless moral, and spiritual support, and good humor during my entire time in school. To my inspiring late father George Godwill, my motivational mother Adele, my best friend, loving and supportive wife Kari Mistkawi who has made my daily life amazingly easy. To my son Tristan, and my daughters, Serene and Amira, I know I cannot make up for lost time during the busy moments, but I promise to be there for you, thank you for your patience. I owe to you everything that I am. Last but not least, to my brother Joseph, and sisters, Mary and Mandy, Thank you for your help and support. I would like to extend many thanks to my parents-in-law Douglas and Barbara Perle for their unconditional help and support for always being their to care for our children as I made progress with school and dissertation work.

I know I am missing a lot of those who have contributed greatly in my life. This is not intentional. Rather my forgetful mind is now betraying. Thank you all! Finally, and above all I give all the glory to the Almighty God who made all this possible. 


\section{TABLE OF CONTENTS}

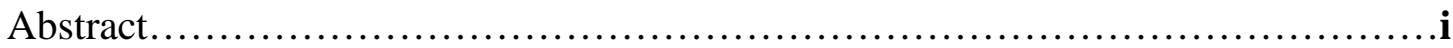

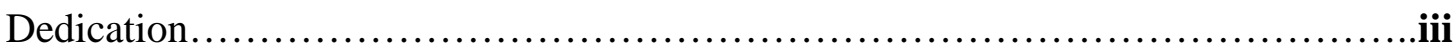

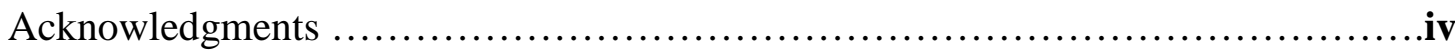

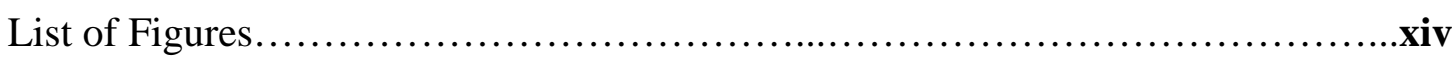

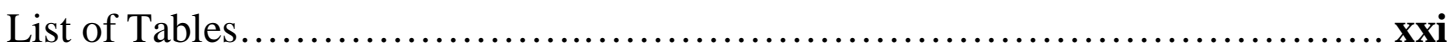

List of Abbreviations ..........................................................

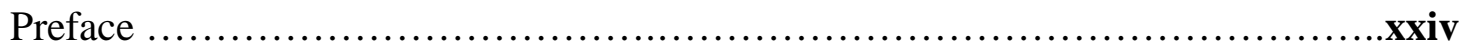

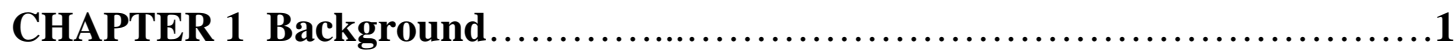

1.1 Historical perspective and Technology trends ...............................1

CHAPTER 2 Semiconductor Processing .........................................................

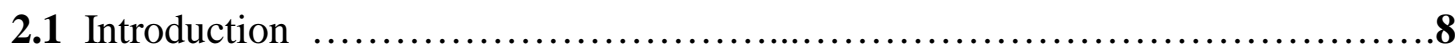

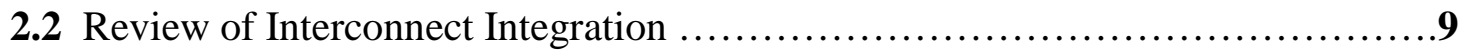

2.2.1 Conventional On-chip Wiring .............................................10

2.3 Interconnects Fabrication Process ............................................

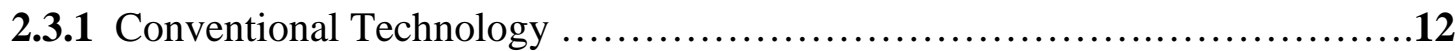

2.3.2 Fabrication of Tungsten Vias ..........................................

2.3.3 Fabrication of Aluminum Alloy Wires ...................................15

2.3.4 Dual-Damascene Copper Technology …..............................17

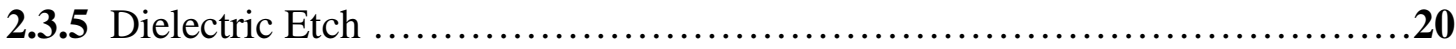

2.3.6 Metal Barrier Deposition ...............................................22

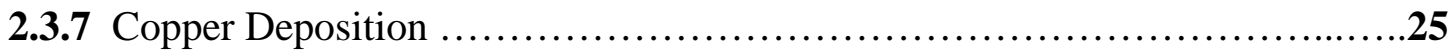


2.3.8 Chemical-Mechanical Polishing …..................................28

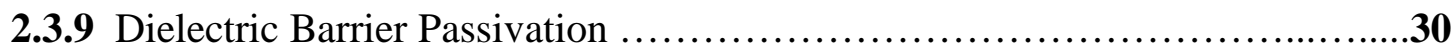

2.4 Interconnects reliability and limitations....................................31

2.4.1 Limitations with Interconnect Scaling....................................31

2.4.2 Interconnect Delays.......................................................

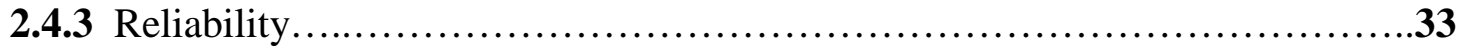

2.4.4 Capacitance Issues.......................................................

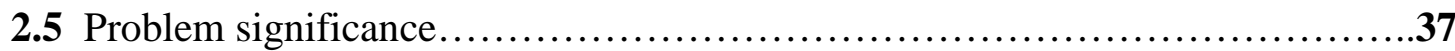

CHAPTER 3 I. Corrosion Behavior of Copper Thin Films in Organic HF

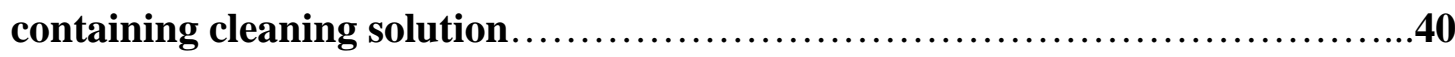

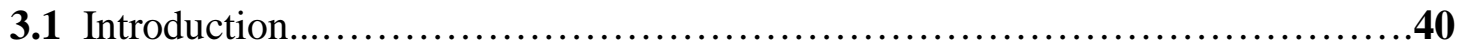

3.2 Copper Rich Residue............................................................

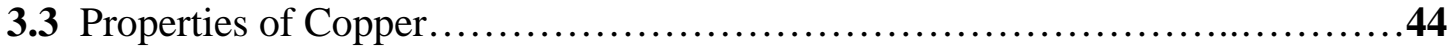

3.3 Principles of corrosion........................................................

3.3.1 Definition....................................................................

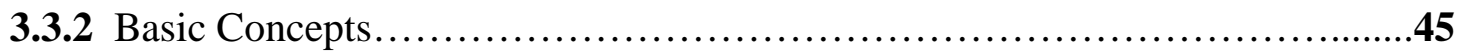

3.3.3 Pourbaix Diagram..........................................................

3.3.4 Pourbaix Diagram Limitations.........................................48

3.3.5 Corrosion Properties of Copper........................................48

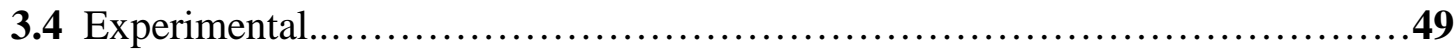

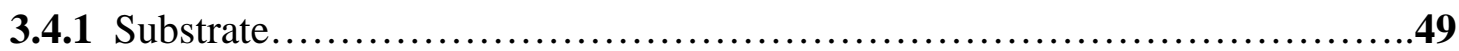

3.4.2 Cleaning solution......................................................... 
3.4.3 Surface Preparation.......................................................

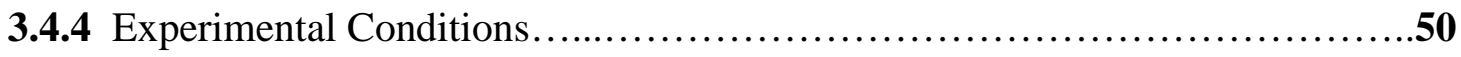

3.4.5 Electrochemical measurements...........................................51

3.4.6 Open-circuit potential experiments..........................................

3.4.7 Potintiodynamic experiments............................................52

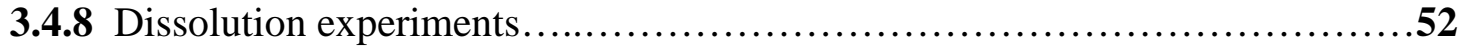

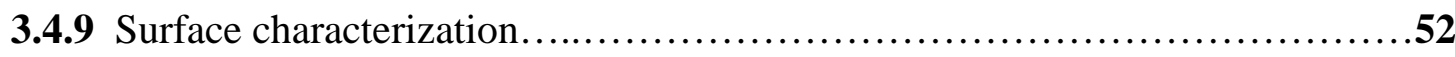

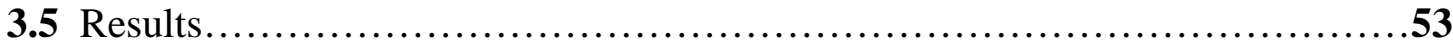

3.5.1 Electrochemical Corrosion Measurements.............................53

3.5.1.1 Open-Circuit Potential Measurements................................53

3.5.1.2 Potentiodynamic Experiments...........................................55

3.5.2 Immersion measurements..............................................56

3.5.2.1 Dissolution experiments in non-deaerated dilute EG3 ......................56

3.5.2.2 Copper Dissolution in aerated dilute EG3 ..............................56

3.5.2.3 Copper Dissolution in deareated dilute EG3 ...........................57

3.5.2.4 Copper Dissolution in non-deareated dilute EG3 in the presence of $\mathrm{H}_{2} \mathrm{O}_{2} \ldots .58$

3.5.2.5 Copper Dissolution in non- deareated dilute EG3 in the presence of BTA....58

3.5.3 Surface characterization.....................................................

3.5.3.1 Atomic Force Microscopy (AFM) .....................................59

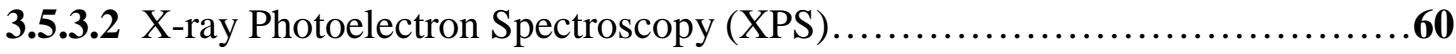

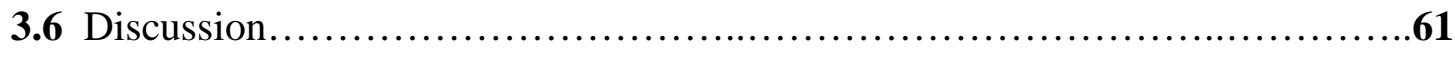

3.6.1 Factors Effect Thin Films Copper Corrosion...............................67 
3.6.2 Copper Corrosion Modes in Interconnects Structures.......................68

3.6.3 Copper Precipitation/Deposition Mechanisms..............................69

CHAPTER 3 II. A Kinetic Study of Copper Thin Film Dissolution..............72

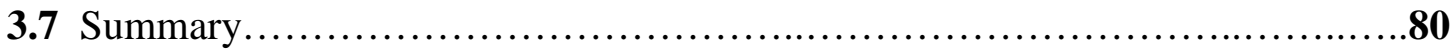

CHAPTER 4. Titanium Assisted Dual Damascene Patterning of Advanced Copper Interconnects and Selective Wet Etch Concepts.......................82

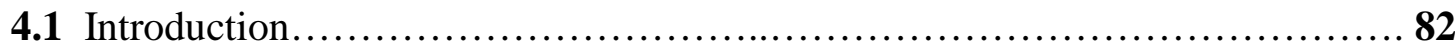

4.2 Titanium Assisted DD patterning of advanced copper interconnects.............85

4.3 Basic Wet Etching Terminology.............................................88

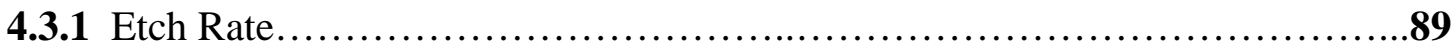

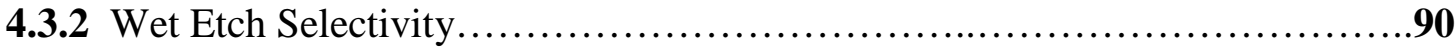

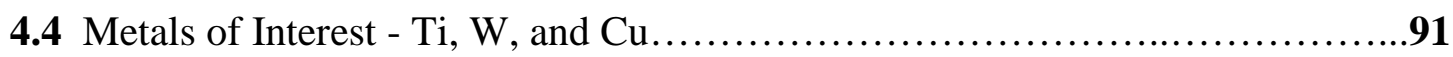

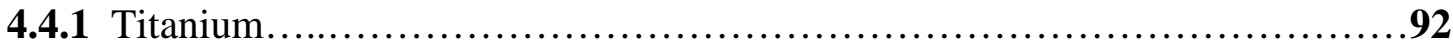

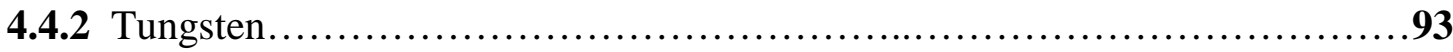

CHAPTER 5 Selective Titanium Wet Etch/Clean Chemical Formulation

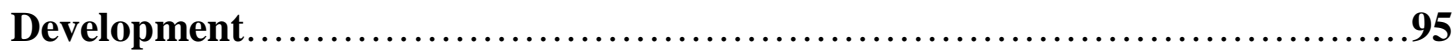

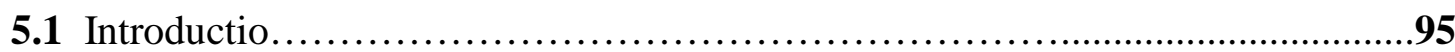

5.2 The Industry's Approach for Removing a Titanium Hard-Mask Film.............96

5.3 Formulation Creation Strategy...............................................

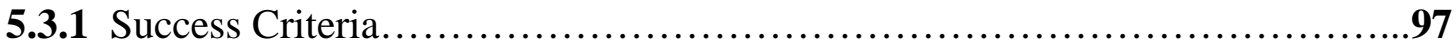

5.3.2 Formulation Design Considerations..........................................97

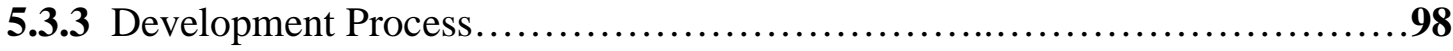


5.4 Fundamentals of Organosilicon and Sol-Gel chemistry.....................99

5.4.1 The Sol-Gel Reaction.............................................102

5.5 Experimental Design..............................................106

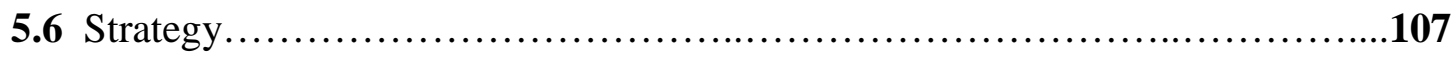

5.6.1 Identifying the Formulation Constituents...........................107

5.6.2 Safety Considerations .............................................

5.6.2.1 Experimental Details..........................................109

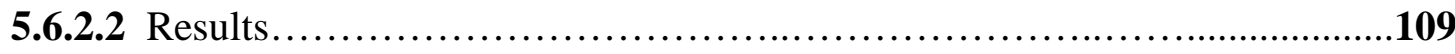

5.6.3 Formulation Synthesis Process...................................111

5.7 Synthesis Details and Reaction Characterization.........................114

5.7.1 Mixing, and Reaction Optimization................................114

5.7.2 Proposed Reaction...............................................115

5.8 Etch Rate Experiments...............................................

5.8.1 Etch Rate Targeting and Optimization.................................116

5.9 DOE analysis and Response Surface Modeling.........................118

$\mathbf{5 . 1 0}$ DOE Results.....................................................119

5.10.1 Prediction and Contour Profiler for $\mathrm{Ti}, \mathrm{SiO}_{\mathrm{x}}$, and $\mathrm{Si}_{3} \mathrm{~N}_{4}$ etch rates...........122

5.11 Enabling the Formulation for Copper...............................127

5.11.1 Copper Corrosion Results......................................128

5.12 Final Formulation Composition...................................131

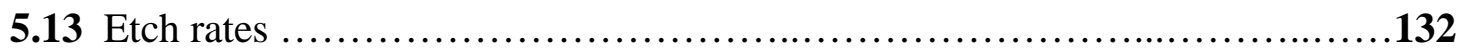

5.14 Effects of Selective Wet Etch Chemistry on DD patterned wafers.............133 


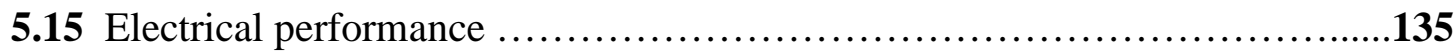

5.16 Time of Flight Secondary Ion Mass Spectrometry (TOF SIMS)...............135

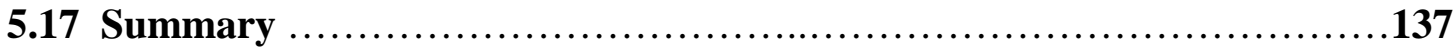

CHAPTER 6 Formulation Characterization and Reaction Kinetics.............141

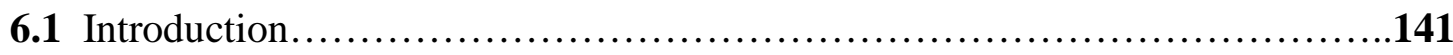

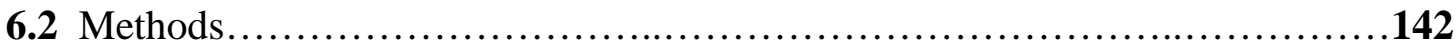

6.2.1 Analytical Methods..................................................

6.2.2 Kinetic Study of Selected Reaction Constituents............................144

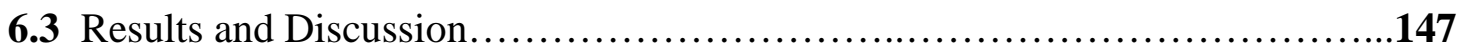

6.5 Kinetic Modeling of Formulation Synthesis...............................154

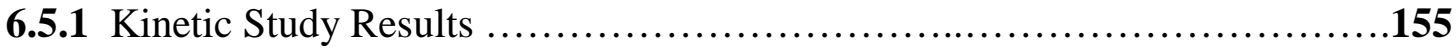

6.6 Model for Ti etch Selectivity ............................................161

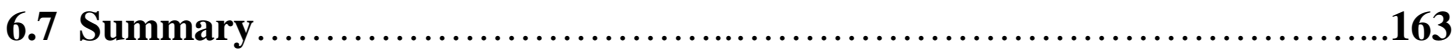

CHAPTER 7 Scalability and Process Control.................................166

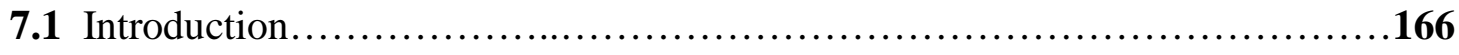

7.2 Systems Designs and Assembly .........................................168

7.2.1 Synthesis in the Bath within the Automated Wet Station.....................168

7.3 Process Control and Stability Results and Discussion .......................171

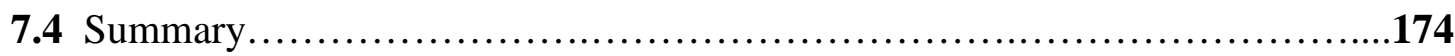

CHAPTER 8 Conclusions and Future Work .............................176

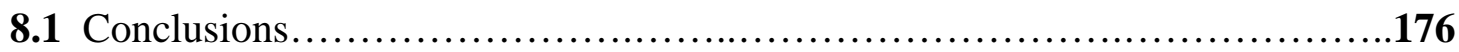

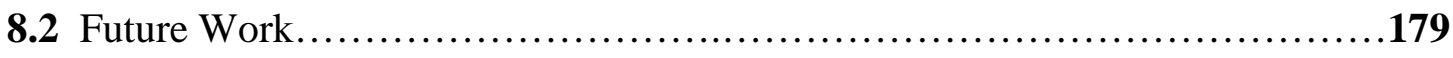




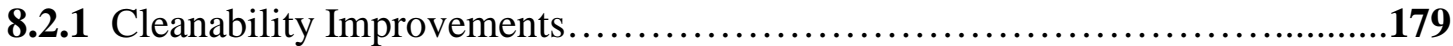

8.2.2 Further Compositional and Kinetic Characterization........................179

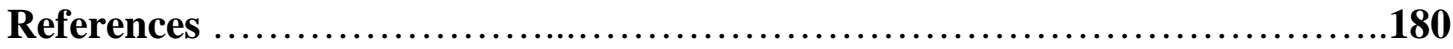

Appendix A Kinetic Analysis for Copper Dissolution..........................191

Appendix B DOE Fitted Model Analysis Outcome for Ti, SiOx, and Si3N4 Etch

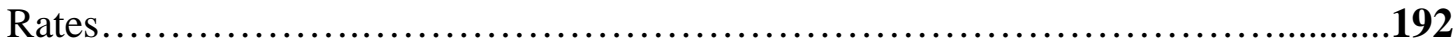

Appendix C List of Publications and Awards ................................197 


\section{List of Figures}

Figure 1.1 Schematic of a transistor and a $\mathrm{Cu}$ wire ...............................

Figure 1.2 Scanning electron micrographs of interconnect architecture with six levels of $\mathrm{Cu}$ wires/vias, W contacts/local interconnects, and $\mathrm{SiO} 2 \mathrm{ILD}$. Demonstrations by

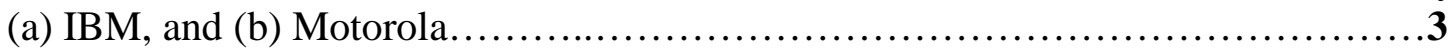

Figure 1.3 Cross section (not to scale) of two transistors, W contacts, and first layer of DD interconnect. Note the sacrificial Ti hard-mask and polymer/residue. Key abbreviations. HM: hard mask, ES: etch stop layer .7

Figure 2.1 Transistor count for Intel microprocessors as a function of year, commonly known as "Moore's Law," which predicts the doubling of transistor count every couple of years.

Figure 2.2 SEM photo of lower levels of interconnect (with dielectric material removed).....................................................................11

Figure 2.3 Sample cross-section of hierarchical wire scaling.....................12

Figure 2.4 Cross-sectional scanning electron micrograph of state-of-the-art $0.25-\mu \mathrm{m}$ CMOS multilevel interconnect technology for high-performance logic..............13

Figure 2.5 Process flow for fabrication of tungsten vias .........................14

Figure 2.6 Process flow for fabrication of aluminum alloy wires .................16

Figure 2.7 Scanning electron micrograph of manufacturable copper interconnect architecture demonstrated by IBM. ..............................................

Figure 2.8 Simplified dual-Damascene process flow for fabricating $\mathrm{Cu}$ Interconnects

Figure 2.9 Dual-Damascene variations for defining wire trenches and via holes: (a) buried etch stop (b) clustered approaches (c) partial via first, (d) full via first, and (e) line first approaches....

Figure 2.10 Comparison of ideal and typical step coverage of a metal barrier

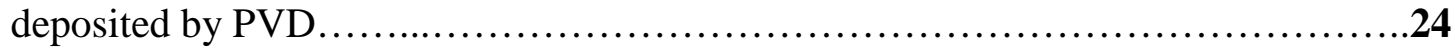

Figure 2.11 Schematic of a $\mathrm{Cu}$ electroplating system..........................27

Figure 2.12 Schematic of a typical chemical-mechanical polishing system............30 
Figure 2.13 Intrinsic gate and interconnect delays as a function of minimum feature size.

Figure 2.14 Impact of line-to-ground and line-to-line coupling on total interconnect capacitance.

Figure 2.15 Power density problem. 36

Figure 3.1 A simple two layers DD interconnect system (not to scale) showing various components such as Diffusion Barrier (DB), Etch Stop (ES) layer, and ILD. 41

Figure 3.2 (a) Effect of copper attack by the wet etch clean chemistry during DD patterning. (b) TEM reference of a functional DD structure.

Figure 3.3 $\mathrm{Cu}$ containing residue observed after the wet etch clean step. (a) optical microscope image. (b) SEM micrograph. 43

Figure 3.4 EDX histogram showing the composition of the residue on the ILD surface.

Figure 3.5 Potential-pH diagram of copper in water system .47

Figure 3.6 Figure 3. Open circuit potential measurements for electrolytic solutions used; (a) in presence of $5 \% \mathrm{H}_{2} \mathrm{O}_{2}$, (b) in an non-deareated (DO $4.5 \mathrm{mg} / \mathrm{L}$ ) and (c) deareated (DO $0.6 \mathrm{mg} / \mathrm{L})$ solutions.

Figure 3.7 Potentiodynamic curves for copper thin films on $\mathrm{Si}$ in cleaning solution containing (a) $\mathrm{H}_{2} \mathrm{O}_{2}$ and under (b) non-deaerated and deaerated conditions .55

Figure 3.8 $\mathrm{Cu}$ dissolution in non-deareated dilute EG3 as a function of time.

Dissolved oxygen concentration was $4.5 \mathrm{mg} / \mathrm{L}$.

Figure 3.9 $\mathrm{Cu}$ dissolution in aerated dilute EG3 as a function of time. Dissolved oxygen concentration was $8.9 \mathrm{mg} / \mathrm{L}$.

Figure 3.10 $\mathrm{Cu}$ dissolution in deareated dilute EG3 as a function of time. Dissolved oxygen concentration was $0.6 \mathrm{mg} / \mathrm{L}$.

Figure 3.11 $\mathrm{Cu}$ dissolution in non-deareated dilute EG3 in the presence of $\mathrm{H}_{2} \mathrm{O}_{2}$ as a function of time. 
Figure 3.12 $\mathrm{Cu}$ dissolution in non-deaerated dilute EG3 in the presence of BTA as a

function of time. 58

Figure 3.13 AFM image of the copper wafer before immersion in dilute EG3......59

Figure 3.14 AFM image of the copper wafer after immersion in dilute EG3.......59

Figure 3.15 XPS spectra of the copper surface before and after surface treatments.

Figure 3.16 XPS spectra of the copper surface before and after wet cleaning solution treatments.

Figure 3.17 Factors influencing copper interconnects corrosion 68

Figure 3.18 Modes of $\mathrm{Cu}$ corrosions that can take place in copper damascene structures.

Figure 3.19 $\mathrm{Cu}$ dissolution in aerated clean solution as a function of time. The DO concentration was $8.9 \mathrm{mg} / \mathrm{L}$. .73

Figure 3.20 $\mathrm{Cu}$ dissolution in aerated and deareated clean solution as a function of time. The DO concentrations were 8.9 and $0.6 \mathrm{mg} / \mathrm{L}$ in the aerated and deareated solutions, respectively. 73

Figure 3.21 DO Concentration in the clean solution in a sealed container as a function of time and $\mathrm{Cu}$ surface area.

Figure 3.22 A simple electrochemical model of the dissolution process showing the cathodic reduction of oxygen coupled with anodic oxidation of $\mathrm{Cu}^{0}$ or $\mathrm{Cu}^{+1}$ that lead to the formation of $\mathrm{Cu}^{2+}$ species in the cleaning solution.........................76

Figure 3.23 $\mathrm{Cu}$ dissolution order kinetics with respect to DO and HF concentrations in (a) and (b) respectively. 78

Figure 4.1 SEM micrograph of an electrically failed via. .84

Figure 4.2 Schematic cross section of an IC showing the Technology miniaturizing trend. 85

Figure 4.3 Schematic cross section of DD patterning steps involving a metal hardmask. 88

Figure 4.4 Potential-pH diagram of Ti in water system. 92 
Figure 4.5 Potential-pH diagram of $\mathrm{W}$ in water system.

Figure 5.1 In sol-gel chemistry, molecular precursors are converted to nanometersized particles, to form a colloidal suspension, or sol. Altering the sol solution conditions produces a gel network. The sol and gel can be processed by various drying methods (shown by the arrows) to develop materials with distinct properties.

101

Figure 5.2 Acid-catalyzed hydrolysis of Silicon Alkoxide.

.104

Figure 5.3 Hydrolysis rate as a function of $\mathrm{pH}$ for $\mathrm{RSi}(\mathrm{OR}) 3+\mathrm{H} 2 \mathrm{O}=\mathrm{RSi}(\mathrm{OR}) 2 \mathrm{OH}$

$+\mathrm{ROH}$ 105

Figure 5.4 Polymerization Time dependence on $\mathrm{pH}$ and $\mathrm{F}^{-}$concentration. 106

Figure 5.5 Background spectrum - prior to precursor (MTES) addition. 109

Figure 5.6 $\mathrm{SiF}_{4}$ calibration spectrum $(10 \mathrm{ppm})$ 110

Figure 5.7 FTIR spectrum after addition of silicon precursor MTES 110

Figure 5.8 Spectra showing $\mathrm{SiF}_{4}$ calibration superimposed on silicon precursor addition 110

Figure 5.9 Process cliffs affecting $\mathrm{Ti}$ selective wet etch clean formulation 113

Figure 5.10 Proposed reaction for the Ti selective etch formulation synthesis. ....116

Figure 5.11 Ti etch rate prediction expression. 120

Figure 5.12 Ti etch rate interaction profiles. HF and MTES concentrations are represented by the $\mathrm{X}$-axis. The red curve corresponds to the low concentration of the complementary component and the blue curve corresponds to the higher concentration

120

Figure 5.13 $\mathrm{SiO}_{\mathrm{x}}$ etch rate prediction expression.

120

Figure 5.14 $\mathrm{SiO}_{\mathrm{x}}$ etch rate interaction profiles. $\mathrm{HF}$ and MTES concentrations are represented by the $\mathrm{X}$-axis. The red curve corresponds to the low concentration of the complementary component and the blue curve corresponds to the higher concentration.............................................................

Figure 5.15 $\mathbf{S i}_{3} \mathbf{N}_{4}$ etch rate prediction expression. .........................121 
Figure 5.16 $\mathrm{Si}_{3} \mathbf{N}_{4}$ etch rate interaction profiles. HF and MTES concentrations are represented by the $\mathrm{X}$-axis. The red curve corresponds to the low concentration of the complementary component and the blue curve corresponds to the higher concentration

Figure 5.17 Prediction Profiler for $\mathrm{Ti}, \mathrm{SiO}_{\mathrm{x}}$, and $\mathrm{Si}_{3} \mathrm{~N}_{4}$ etch rates from the fitted model. 123

Figure 5.18 Etch rate DOE/response surface for HF, MTES, and water concentrations.

Figure 5.19 Etch rate DOE showing process target and process window...........125

Figure 5.20 Three adsorption coordination of BTA. .......................128

Figure 5.21 Equilibrium Rest Potential for two different BTA concentrations. .....129

Figure 5.22 Linear Polarization measurements on $\mathrm{Cu}$ surface indicating very low corrosion. ..............................................................

Figure 5.23 Tafel Polarization - Copper etch rate $=0.04 \AA / \mathrm{min} . \ldots \ldots \ldots \ldots \ldots . . . .131$

Figure 5.24 SEM micrograph of Copper surface before and after processing in the formulation in (a) and (b) respectively. 131

Figure 5.25 Etch rate results in the Ti selective wet etch formulation at $60{ }^{\circ} \mathrm{C}$. The maximum error in the ER determination is approximately $5 \%$. 132

Figure 5.26 SEM micrograph of DD patterned wafers after the break-through plasma etch process and before treatment with the Ti selective wet etch chemistry..... 133

Figure 5.27 SEM micrograph of DD patterned wafers after the break-through plasma etch process and after treatment with the Ti selective wet etch chemistry...........134

Figure 5.28 Resistance distribution of 65,000 vias chain for wafers cleaned with the developed Ti strip/clean chemistry. 135

Figure 5.29 Depth profile determination of titanium concentration as a function of depth. 136

Figure 5.30 Evolution of reflectance images as a function of etch time. ...........136

Figure 5.31 Reflectance images on patterned wafers. .......................137 
Figure 6.1 Calibration Curve constructed using the silicon standard solution.......146

Figure 6.2 ${ }^{1} \mathrm{H}$ NMR spectrum of wet etch/clean formulation. ...................148

Figure 6.3 ${ }^{1} \mathrm{H}$ NMR spectrum. Note multiple peaks in the methyl region...........148

Figure 6.4 ${ }^{19}$ F NMR spectrum of formulation. .............................. 149

Figure $6.5{ }^{19} \mathrm{~F}$ NMR spectrum of pure HF. ................................149

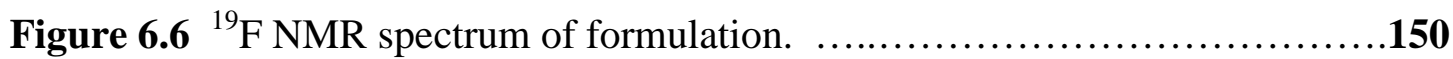

Figure 6.7 Entire ${ }^{19} \mathrm{~F}$ NMR spectrum of formulation is shown for reference........150

Figure 6.8 GC-MS Spectrum indicates a grouping of peaks that are separated by 77

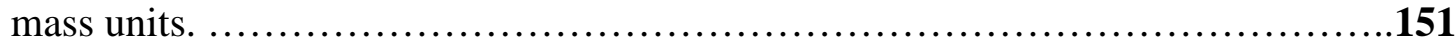

Figure 6.9 MALDI-MS spectrum for the formulation. .......................152

Figure 6.10 MALDI lower mass spectrum of the formulation..................152

Figure 6.11 UV/VIS and MS spectrums in (a) and (b) respectively, indicating the presence of BTA in the UV/VIS region. ................................

Figure 6.12 ELSD in (a) and MS spectrums in (b) confirming the presence of BTA

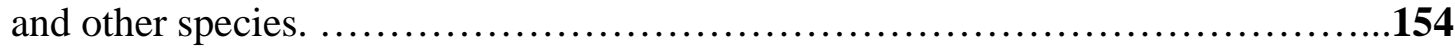

Figure 6.13 $\mathrm{SiO}_{2}$ ER Kinetic Order with Respect to HF Concentration. ...........157

Figure 6.14 HF concentration as a function of synthesis time shows exponential decay, with time constant of 29 minutes. ...................................

Figure 6.15 Ti ER Kinetic Order with Respect to HF Concentration...............160

Figure 6.16 $\mathrm{Ti} / \mathrm{SiO}_{2}$ selectivity with Respect to $\mathrm{HF}$ Concentration..............161

Figure 6.17 Schematic diagram of $\mathrm{Ti}$ dissolution in the wet etch/clean formulation. 163

Figure 7.1 Schematic of the formulation synthesis system within the automated wet station. Dilute HF bath and the MTES delivery components are shown ............168

Figure 7.2 Schematic diagram of 3000 gallon reactor and its components. ........170 
Figure 7.3 The syntheses model with respect to HF concentration and silicon oxide etch rate. ............................................................

Figure 7.4 Reaction profile with respect to HF concentration and reaction time at

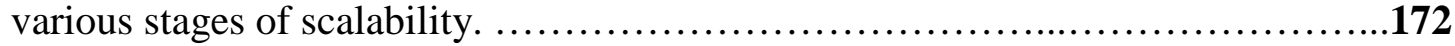

Figure 7.5 $\mathrm{SiO}_{2}$ etch rate process control charts for various batches from the

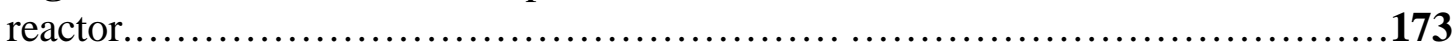

Figure 7.6 HF concentration process control charts for various batches from the

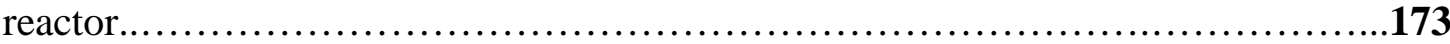

Figure 7.7 $\mathrm{F}^{-}$Concentration process control chart for a number of Batches.........174 


\section{List of Tables}

Table 2.1 Interconnect Design Rules for $0.25-\mu \mathrm{m}$ Technology ...................17

Table 2.2 Comparison of Dual-Damascene Dielectric Etch Approaches.............22

Table 2.3 Wiring pitch and aspect ratio are the geometries of interest for cleaning

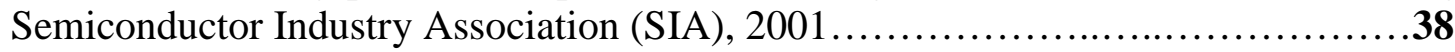

Table 3.1 Reactions showing $\mathrm{CuF}_{3}^{-}$formation and possible $\mathrm{Cu}$ deposition mechanism. ................................................................

Table 4.1 Some important characteristics of titanium and tungsten................91

Table 5.1 Etch rates as a function of formulation constituents concentrations........118

Table 5.2 Etch rates as a function of concentrations by volume and

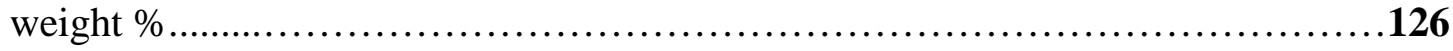

Table 5.3 Composition of the Ti selective wet etch formulation. ...................132

Table 5.4 Etch rate results of the various thin film materials in the Ti selective Wet

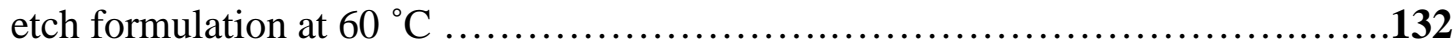

Table 6.1 $\mathrm{H}_{2} \mathrm{SiO}_{3}, \mathrm{H}_{2} \mathrm{SiF}_{6}$, and $\mathrm{HF}$ concentrations, and Etch rates of $\mathrm{SiO}_{2}$ and $\mathrm{Ti}$ determined in real time while synthesis is in progress. .......................156 


\section{List of Abbreviations}

Acronym

AFM

ARC

BEOL

BTA

BTB

CD

CDO

CMOS

CMP

CVD

DB

DCCD

DD

DOE

EB

EDX

ELSD

EP

ER

ES

FCCD
Elaboration

Atomic Force Microscopy

Anti-Reflective Coating

back-end-of-line

Benzotriazole

Bromothymol Blue

Critical Dimension

Carbon Doped Oxide

Complimentary Metal Oxide Semiconductor

Chemical-Mechanical Polishing

Chemical Vapor Deposition

Diffusion Barrier

Develop Check Critical Dimension

Dual Damascene

Design of Experiments

Etch Bias

Energy Dispersive X-ray

Evaporating Light Scattering Detector

Electro Plating

Etch Rate

Etch-Stop

Final Check Critical Dimension 


\begin{tabular}{|c|c|}
\hline FEOL & Front-End-of-Line \\
\hline FTIR & Fourier Transform Infrared Spectroscopy \\
\hline GCMS & Gas Chromatography Mass Spectrometry \\
\hline $\mathrm{HM}$ & Hard-Mask \\
\hline HVM & High Volume Manufacturing \\
\hline IC & Integrated Circuit \\
\hline ILD & Inter Layer Dielectric \\
\hline ISE & Ion Selective Electrode \\
\hline MALDI MS & Matrix-Assisted Laser Desorption Mass Spectrometry \\
\hline MHM & Metal Hard-Mask \\
\hline MTES & Methyltriethoxysilane \\
\hline PVD & Physical Vapor Deposition \\
\hline RIE & Reactive Ion Etch \\
\hline RSM & Response Surface Methodology \\
\hline SEM & Scanning Electron Microscope \\
\hline $\mathrm{SiOF}$ & Fluorine Doped Silicon Oxide \\
\hline SIV & Stress-Induced Voiding \\
\hline TEM & Transmission Electron Microscopy \\
\hline TOF SIMS & Time of Flight Secondary Ion Mass Spectroscopy \\
\hline ULSI & Ultra Large Scale Integration \\
\hline VOC & Volatile Organic Compound \\
\hline XPS & X-ray Photoelectron Spectroscopy \\
\hline
\end{tabular}




\section{Preface}

Aluminum alloys, tungsten and $\mathrm{SiO}_{2}$ insulators have been the materials of choice for interconnect systems since the dawn of the Integrated Circuit (IC) era. These materials were convenient to process using subtractive etch processes for metal line patterning. However, as ICs have relentlessly marched down the path towards smaller geometries in the pursuit of low power consumption, increased speed and high integration density, the $\mathrm{Al} / \mathrm{W} / \mathrm{SiO}_{2}$ interconnect system became a limiting factor. Today in advanced interconnect systems; Copper and Tungsten are the choice metallization for Ultra Large Scale Integration (ULSI). Tungsten is only utilized for local interconnects (first level metallization), contacts to transistor components, gate, source, and drain. Copper wiring (interconnects) is employed in all other layers and can be up to 12 metallization levels of copper wires, where one level communicates with another through conductive copper vias, See figure 1.1. The replacement of aluminum alloys by copper, as the metal of choice, mandated prominent changes in integration, metallization, and patterning process technologies. For instance, the introduction of copper in semiconductor devices has brought attention to the phenomena of corrosion that must be circumvented for optimal device performance, reliability, and longevity.

Ti hard masks have been recently adopted to enable the integration schemes of advanced interconnect technologies. The utilization of a metal HM has been a leading approach to allow for patterning smaller dimensions, preventing profile distortion, and in achieving high aspect ratio structures, which has lead to further scaling, and 
increased component density in ULSI devices. To fabricate Ti HMs on chip surface increasingly sophisticated selective etching/cleaning chemical solutions are needed. Further more these chemical formulations must be compatible towards copper, tungsten and low-k dielectrics used in advanced devices.

The work presented in this dissertation evolved from the study and characterization of the corrosion behaviors of copper thin films. Subsequently, copper corrosion work/learnings were applied to develop a novel Ti selective wet etch/clean chemical formulation that has now enabled future ULSI technologies. The developed formulation is capable of selectively striping patterned Ti HM thin films, while suppressing the etch rates of $\mathrm{W}, \mathrm{Cu}, \mathrm{SiO}_{\mathrm{x}}$, silicon carbide $(\mathrm{SiC})$, silicon nitride $\left(\mathrm{Si}_{3} \mathrm{~N}_{4}\right)$, and carbon doped silicon oxide (CDO). Furthermore, its able to remove post plasma etch polymer/residue typically generated in usual semiconductor processing.

The Following is a brief outline of the thesis. A historical perspective and technology trends appear in chapter 1 followed by an overview of semiconductor processing and a review of interconnect integration in chapter 2. Chapter 3 presents a detailed study of the corrosion behaviors of copper thin films in organic HF containing cleaning solution for semiconductor applications. Chapters 4 and 5 give a detailed description of the Selective $\mathrm{Ti} \mathrm{HM}$ chemical formulation development and performance. Chapter 6 provides a comprehensive analytical study that characterizes the formulation composition. In chapter 7, a kinetic study of the formulation synthesis is described. In concluding, Chapter 8 describes the scalability work undertaken to enable the wet etch/clean formulation for high volume manufacturing. 


\section{CHAPTER 1}

\section{Background}

\subsection{Historical perspective and Technology trends}

Some of the greatest innovations of the past half-century have involved the integrated circuit (IC) industry. The trend is likely to continue either directly from semiconductor technology or through its incorporation in the biosciences and other disciplines. Chemistry plays an important role in the continued development of ICs and related devices processing. Indeed, current limitations in existing ICs such as the microprocessor lie not in device or circuit design, but in our ability to manufacture these reliably and affordably on a large scale. At no other time in the history of the industry have more new materials been introduced/required at the same time. At the core of many of the obstacles facing the microelectronics industry are issues that can be only solved through knowledge and applying basic chemical principles, including thermodynamics, quantum mechanics, surface chemistry, and chemical kinetics. Corrosion processes and selective wet etching/cleaning understanding lie at the heart of the various challenges that must be overcome to fabricate reliable high performance microprocessors of the future.

As the number of transistors per microprocessor is approaching $1 \mathrm{billion}$, high degree of complexity has evolved in electrically connecting them. At the turn of the millennium, copper replaced aluminum as the interconnect material in ULSI devices. The introduction of copper to construct interconnect systems required the adoption of 
a process known as Dual Damascene (DD). The simplest possible system which involves only one device (transistor) is illustrated in Figure 1.1.

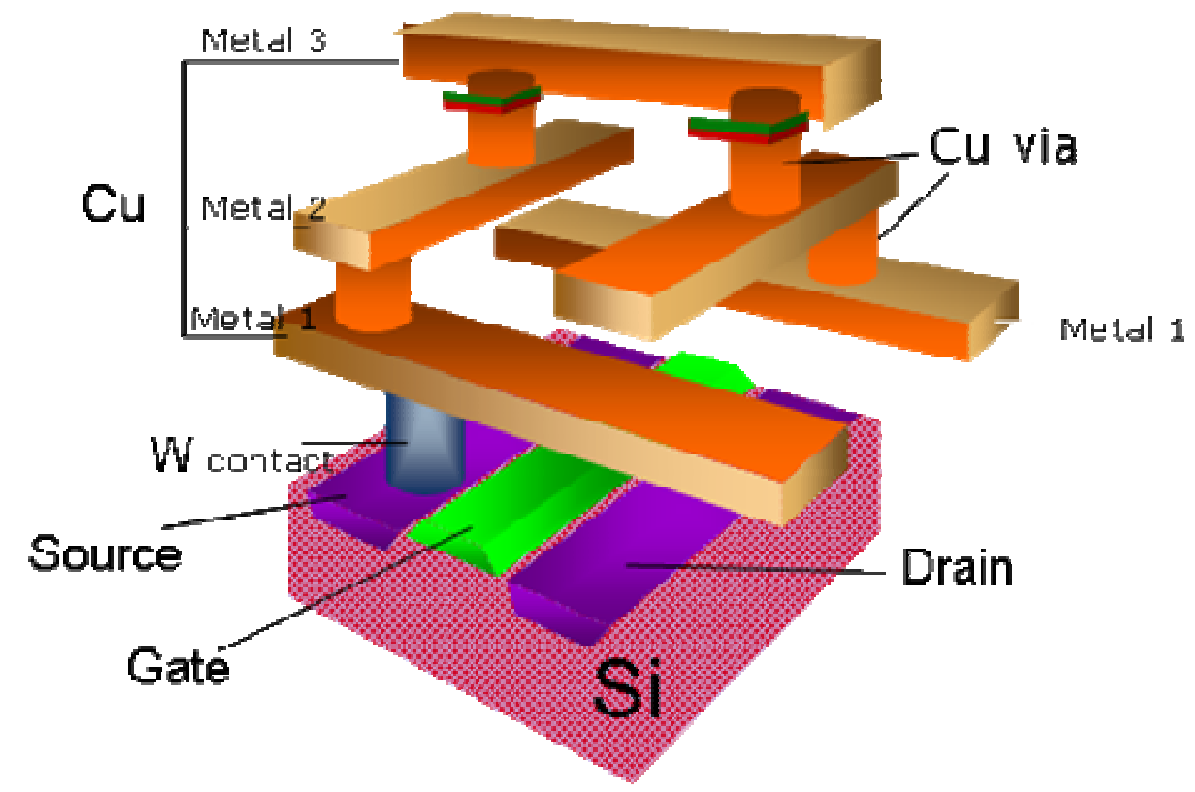

Figure 1.1 Schematic of a transistor and a $\mathrm{Cu}$ wire.

Electrical signals to the source, drain, and gate of the transistor are transported over a three dimensional superhighway layered in multilayered structures connected through vias. Modern microprocessor may have up to 12 layers of metals. In between the layers and lines is insulating materials, low k interlayer dielectric (ILD).

Due to it's high conductivity, $\mathrm{Cu}$ as interconnect material was actively explored for many years, ${ }^{1,2}$ but its feasible production technology was not demonstrated until the late 1990's. In September 1997, IBM and Motorola declared their intentions to incorporate $\mathrm{Cu}$ with oxide $\left(\mathrm{SiO}_{2}\right)$ in their next generation $\mathrm{CMOS}$ logic technologies (Figure 1.2) ${ }^{3,4}$. Shortly after, the performance advantage of $\mathrm{Cu}$ 
interconnects was demonstrated in high-speed microprocessors ${ }^{5}$. What came as a surprise is the claim that this performance improvement is achieved at a lower $\operatorname{cost}^{6}$.

(a)

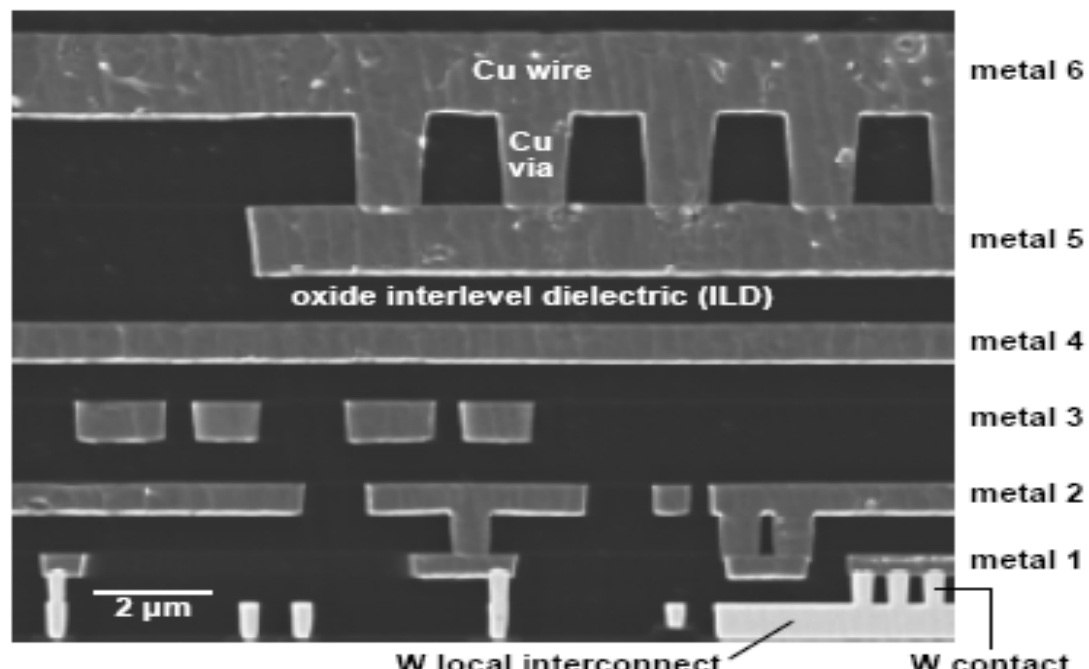

(b)

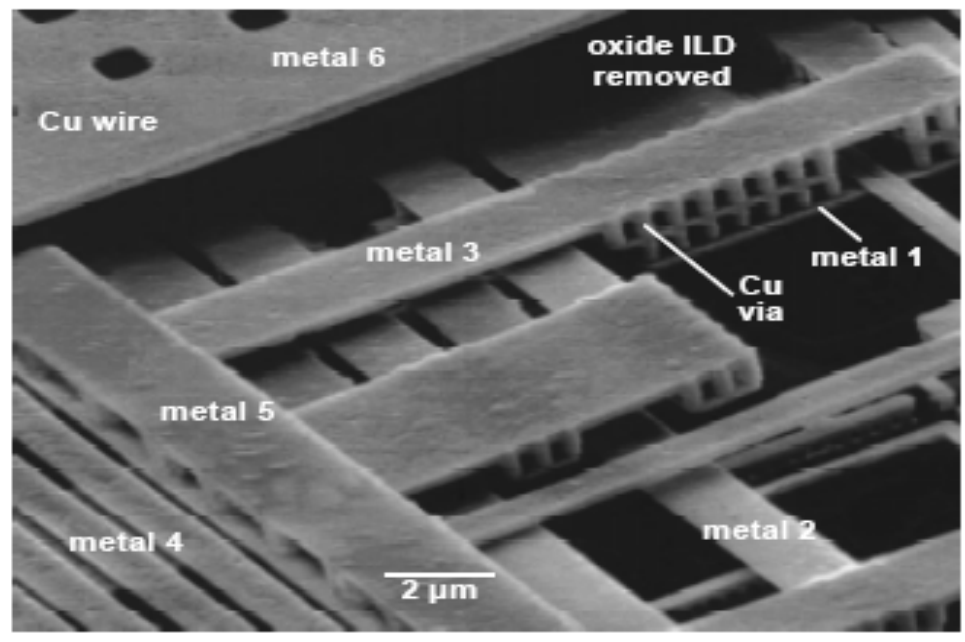

Figure 1.2 Scanning electron micrographs of interconnect architecture with six levels of $\mathrm{Cu}$ wires/vias, W contacts/local interconnects, and SiO2 ILD. Demonstrations by (a) IBM, and (b) Motorola.

The concept of dual damascene (DD) was introduced to the patterning of copper interconnects in which copper is poured into patterned trenches and vias within the ILD $^{7}$. During the metallization process, the DD structures patterned in the ILD 
are filled with copper utilizing an electroplating process which has been the leading approach for the integration of copper interconnects in advanced ULSIs. In addition, polishing technologies had to be introduced into the interconnect manufacturing process to remove excess plated copper by utilizing a chemical-mechanical polishing (CMP) process. Plasma (dry) etch process is commonly used to pattern the vias and trenches into the ILD. The plasma etch process is anisotropic in nature and has the ability to produce vertical sidewalls which is essential for successful DD integration. However, the plasma etch process causes the formation of polymer/residue resulting from the plasma gases reaction with the etched materials. In order to obtain reliable IC devices with low interconnect resistance, the polymer/residue on the sidewalls and bottom of the vias must be removed prior to the next process step. There have been many approaches explored to remove the plasma etch residues. These include the use of $\mathrm{O}_{2}, \mathrm{NF}_{3} / \mathrm{Ar}$ and $\mathrm{He} / \mathrm{H}_{2}$ plasma chemistries to clean polymeric residues, ${ }^{8,9,10,11,12}$ as well as the use of solutions such as aqueous dilute $\mathrm{HF}^{13}$ and a variety of other formulation designs.

As fabrication technology advances to length scale in sub $50 \mathrm{~nm}$, the concept of using a sacrificial hard-mask (HM) that is completely removed at the end of the DD patterning process was presented ${ }^{14}$. The use of a sacrificial HM during DD patterning has emerged as a potential method for controlling via flare ${ }^{15}$. Additional key benefits that have been realized as a result of utilizing a hard-mask are: 1) allows for a wide process window for etch rate control which enhances etch selectivity, 2) permits for significant improvement with respect to etching high aspect ratio structures with 
robust via and trench profiles, 3) critical dimension (CD) control which allows for acceptable Etch Bias (EB) of the critical dimension, where

$$
\mathrm{EB}=\mathrm{FCCD}-\mathrm{DCCD}
$$

where DCCD and FCCD are the critical dimension at the lithography develop check and etch final check steps, respectively, 4) protects the ILD material, porous ILD in particular, from damage induced by the plasma during the dry etch process, and 5) acts as an interface between the ILD and photoresist during both the via and trench patterning steps. Dry etch selectivity between photoresist and ILD is difficult to attain, especially when polymer ILDs are used. Selectivity is defined in detail in chapter 4. The etch strategy relies on consuming most, if not all, of the photoresist during the via/or trench etch process. Under such circumstances, the presence of the hard-mask prevents the via and trench etch chemistry from attacking the polymer ILD in the masked region of the wafer when the photoresist is consumed. A dual hard-mask approach, ${ }^{16,17,18}$ and triple hard-mask integration schemes ${ }^{19,20,21}$ are also proposed for patterning DD structures in polymer and hybrid ILDs.

To fully realize the aforementioned benefits of employing a hard-mask, a metallic hard-mask must be used. Metallic hard-masks are replacing conventional inorganic hard-masks such as $\mathrm{SiO}_{2}$ and $\mathrm{SiC}$ in ULSIs patterning schemes due to their chemical nature which provides significant improvements ${ }^{22}$ with respect to plasma etch selectivity. It is anticipated that conventional inorganic hard-masks $\left(\mathrm{SiO}_{2}\right.$ and SiC) cannot be implemented in future DD integration schemes of advanced interconnect systems due to their low selectivity and severe faceting generated during 
the dielectric etch processes. New metallic hard-masks have been introduced as potential candidates to replace the conventional $\mathrm{SiO}_{2}$ and $\mathrm{SiC}$ inorganic hard masks. Metallic and composite materials such as $\mathrm{Ti}, \mathrm{TiN}, \mathrm{TaN}$, etc... have a different chemical nature than dielectric material and therefore exhibit better hard mask capabilities ${ }^{23}$ (higher selectivity and less faceting) than conventional hard masks.

Figure 1.3 illustrates the integration of a sacrificial $\mathrm{Ti}$ hard-mask in the patterning scheme of advanced DD interconnect structures. The Figure shows the polymer/residue that results from the plasma etch process after removing the etch-stop (ES) protective layer to expose tungsten. The various elements of a simple device containing two transistors and two layers of interconnects are also illustrated. SiN or $\mathrm{SiC}$ are typically used as ES layers in the integration schemes of interconnect systems. The primary function of this layer is to act as a diffusion barrier that inhibits copper from diffusing into the ILD. Most of the material specification stems from this key functionality. In addition, the ES layer protects the underlying metal from wet and dry chemicals used during the patterning process. The polymer/residue can be composed of a complex inorganic, organic, or organo-metallic compounds that encompass mixtures of elements such as $\mathrm{W}_{\mathrm{x}} \mathrm{F}_{\mathrm{y}} \mathrm{N}_{\mathrm{z}} \mathrm{C}_{\mathrm{n}} \mathrm{Ti}_{\mathrm{m}} \mathrm{O}_{\mathrm{j}}$ at the first level interconnects (local wiring), and $\mathrm{Cu}_{\mathrm{x}} \mathrm{F}_{\mathrm{y}} \mathrm{N}_{\mathrm{z}} \mathrm{C}_{\mathrm{n}} \mathrm{Ti}_{\mathrm{m}} \mathrm{O}_{\mathrm{j}}$ is expected for the upper layers interconnects where only copper metallization is used. To complicate matters further, the $\mathrm{Ti}_{\mathrm{x}} \mathrm{F}_{\mathrm{y}} \mathrm{C}_{\mathrm{z}}$ residues seem to become larger and denser as a function of plasma etch time ${ }^{24}$. Therefore, novel cleaning approaches are needed to selectively remove the metal hard-mask and heavy etch polymer/residues after patterning. 


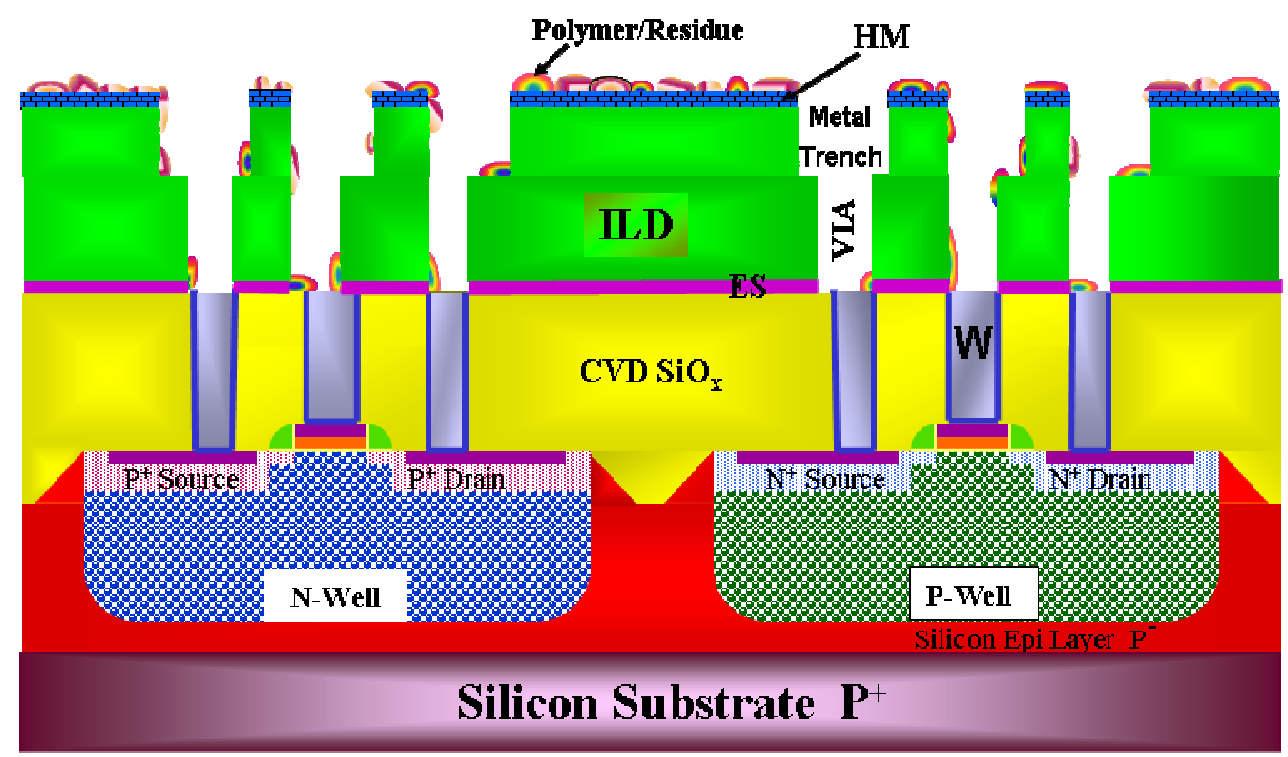

Figure 1.3 Cross section (not to scale) of two transistors, W contacts, and first layer of DD interconnect. Note the sacrificial Ti hard-mask and polymer/residue. Key abbreviations. HM: hard mask, ES: etch stop layer.

When the etch-stop layer is removed, $\mathrm{W}$ at the local interconnects level and $\mathrm{Cu}$ for all other interconnect levels become exposed to the wet etch chemical solution in the subsequent process step. Therefore, it is imperative that $\mathrm{Cu}, \mathrm{W}$, and ILD compatible wet etch chemical formulations are employed. In addition, the presence of $\mathrm{Cu}$ and $\mathrm{W}$ in the chemical solution resulting from the metal corrosion/dissolution during the etch/clean process must be minimized or prevented. $\mathrm{Cu}$ and/or $\mathrm{W}$ in the chemical solution present reliability and yield risk to IC devices since they can deposit/precipitate onto the wafer surface. The work presented in this dissertation explored the corrosion behaviors of copper thin films in an organic HF containing solution. Furthermore, the invention of a selective $\mathrm{Ti}$ wet etch/clean formulation is revealed. 


\section{CHAPTER 2}

\section{Semiconductor Processing}

\subsection{Introduction}

The manufacturing of ICs today typically occurs by means of more than 400 processing steps ${ }^{25}$ such as ion implantation, deposition, lithography, and etching. Precise control of these operations is critical for high-yield production of defect-free products. Contamination is a major source of these defects, currently accounting for $50 \%$ or more of yield losses in IC manufacturing. Accordingly, among the most numerous processing steps are cleaning operations, often numbering more than 100 . Cleaning, in general, aims at selectively removing an unwanted thin layer to yield a clean surface having desirable properties. These unwanted substances may include organic films such as photoresist, inorganic materials such as metals, metal oxides or metal salts, or residues and particulates such as those originating from plasma reactors. Semiconductor cleaning specifically encompasses surface preparation and modification, residue removal, thin film stripping, particle removal, rinsing, and drying.

Since the first development of manufacturing processes for solid-state devices and ICs, cleaning has been performed primarily using liquid phase processes. In the semiconductor industry, there are two main regimes; they are designated as FrontEnd-of-Line (FEOL) or Back-End-of-Line (BEOL). In FEOL transistors are fabricated through selective doping of the silicon substrate. The standard clean for 
decades for FEOL has been the RCA clean ${ }^{26}$ or modifications thereof. The RCA clean is a multistep process comprised of an $\mathrm{NH}_{4} \mathrm{OH} / \mathrm{H}_{2} \mathrm{O}_{2} / \mathrm{DIW}$ (deionized water) known as Standard Clean 1 (SC-1), $\mathrm{HCl} / \mathrm{H}_{2} \mathrm{O}_{2} / \mathrm{DIW}$ known as Standard Clean 2 (SC-2), dilute HF dip and DIW rinse steps. Other FEOL formulations that have been used include Piranha clean (sulfuric acid/ $\left.\mathrm{H}_{2} \mathrm{O}_{2} / \mathrm{DIW}\right)^{27}$ and similar formulations.

BEOL refers to all processing beyond the first deposition of metal and includes fabrication of the multiple levels of interconnect wiring and insulators. Interconnects are generally fabricated by blanket deposition of a material followed by selective plasma etching. Etching by means of plasmas is highly anisotropic, and is more precise. Therefore, plasma-etching processes are used in critical steps of fabrication. Plasma processes are not without their shortcomings, Substrate modification/damage, contamination, and, polymer/residue are common.

\subsection{Review of Interconnect Integration}

The investigations to be presented in subsequent chapters assume a basic familiarity of interconnect process technologies. This section of chapter 2, provides an overview of interconnect integration, emphasizing key features in both conventional $\mathrm{Al}$ and Damascene $\mathrm{Cu}$ technologies.

In conventional silicon ULSI technologies, the interconnects are formed after front end processing. The increase in transistor density (Figure 2.1) is a challenge considering that each device must communicate with other devices, each circuit block needs to talk to other circuit blocks, and so on up the hierarchy. The interconnections required to support this communication grow increasingly more complex. 
Furthermore, due to the higher clock frequencies, these interconnections need to support higher bandwidths.

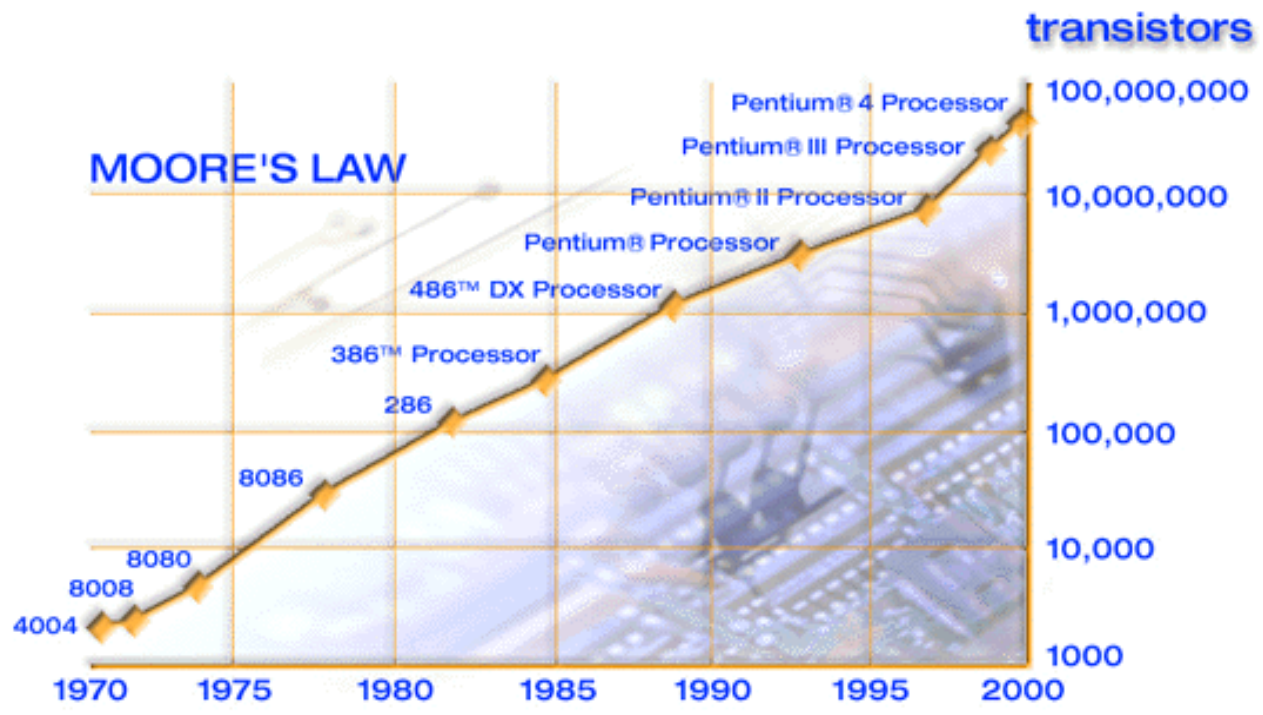

Figure 2.1 Transistor count for Intel microprocessors as a function of year, commonly known as "Moore's Law," which predicts the doubling of transistor count every couple of years ${ }^{28}$.

It is easy to imagine what problems may occur if this congestion is not addressed. The problem is as familiar as the rush-hour traffic encountered in daily commuting. The obvious solutions of building more highways and widening lanes are prohibitively expensive. Similarly, adding more metal levels and increasing chip size to accommodate more interconnects can significantly add to the overall cost. Interconnect technology designs for high-performance digital systems is a growing problem that needs to be addressed early on in a design flow ${ }^{29}$.

\subsubsection{Conventional On-chip Wiring}

On-chip wires have always been necessary to connect circuits together. While the metal and dielectric materials are undergoing changes, the wires largely have 
remained the same in function and usage. One significant difference is in their growing number. Figure 2.2 shows a Scanning Electron Microscope (SEM) image of local interconnect with the dielectric material removed ${ }^{30}$. These on-chip wires are thin and narrow with cross-sectional dimensions typically less than half of a micron. On the local level, they connect transistors that are a few microns apart. On the global level, they must span across an entire chip.

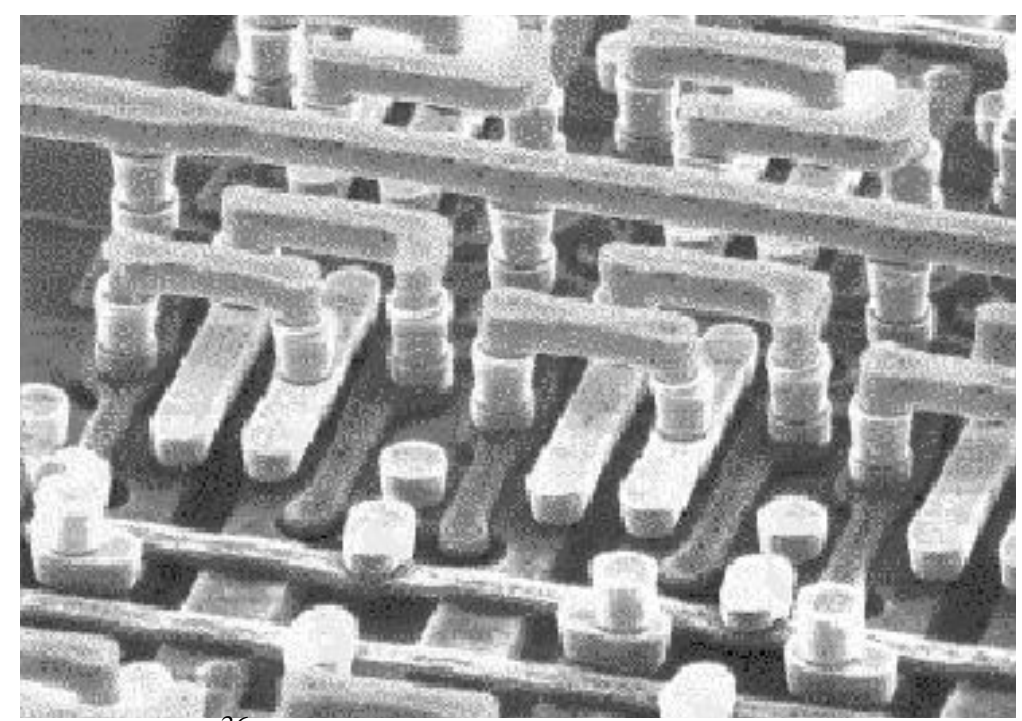

Figure 2.2 SEM photo ${ }^{36}$ of lower levels of interconnect (with dielectric material removed).

A typical wiring hierarchy is shown in Figure 2.3, which shows a cross-section of an advanced hierarchical wiring scheme using copper metallization ${ }^{31}$. The lower levels of interconnect are thinner and are used in local routing. Intermediate layers are of medium thickness and used for semi-global routing. Finally, the top layers are the thickest and are used for global routing. Alternating levels of interconnect are customarily laid out in orthogonal directions to minimize cross-talk between adjacent levels. Furthermore, this convention helps to simplify routing. 


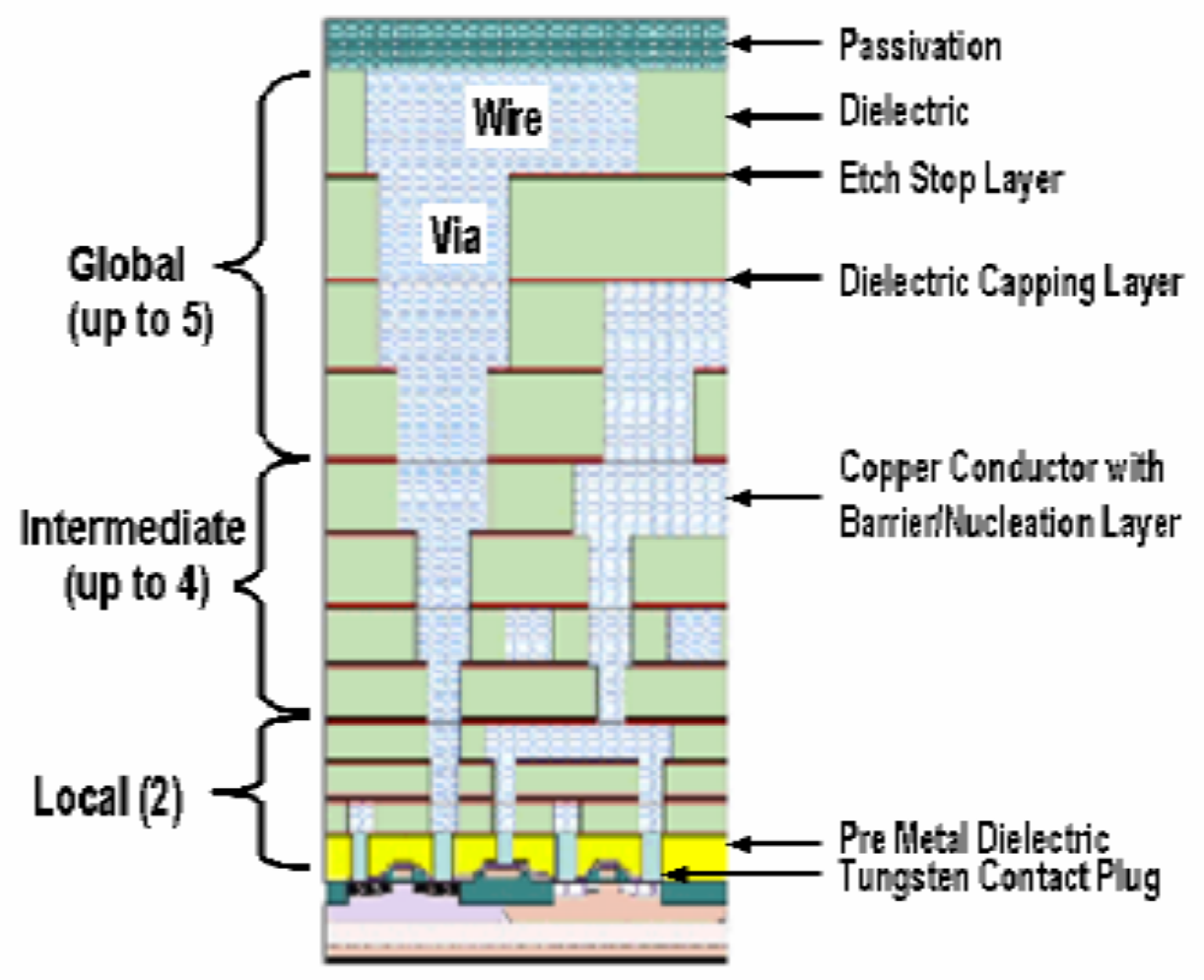

Figure 2.3 Sample cross-section of hierarchical wire scaling ${ }^{37}$.

\subsection{Interconnects Fabrication Process}

\subsubsection{Conventional Technology}

The state-of-the-art $0.25-\mu \mathrm{m}$ interconnect system, shown in Figure 2.4, illustrates the integration of conventional $\mathrm{Al}$ metallization. The example consists of five levels of aluminum alloy wires and tungsten vias also called plugs or studs embedded in oxide $\left(\mathrm{SiO}_{2}\right)$. Integration success is largely attributed to the processes that maintain excellent planarity after fabricating each and every via and wire level. The absence of topography helps to mitigate the fundamental depth-of-focus limitation of high-resolution lithography and avoid reliability problems such as metal line breaks over dielectric steps. 


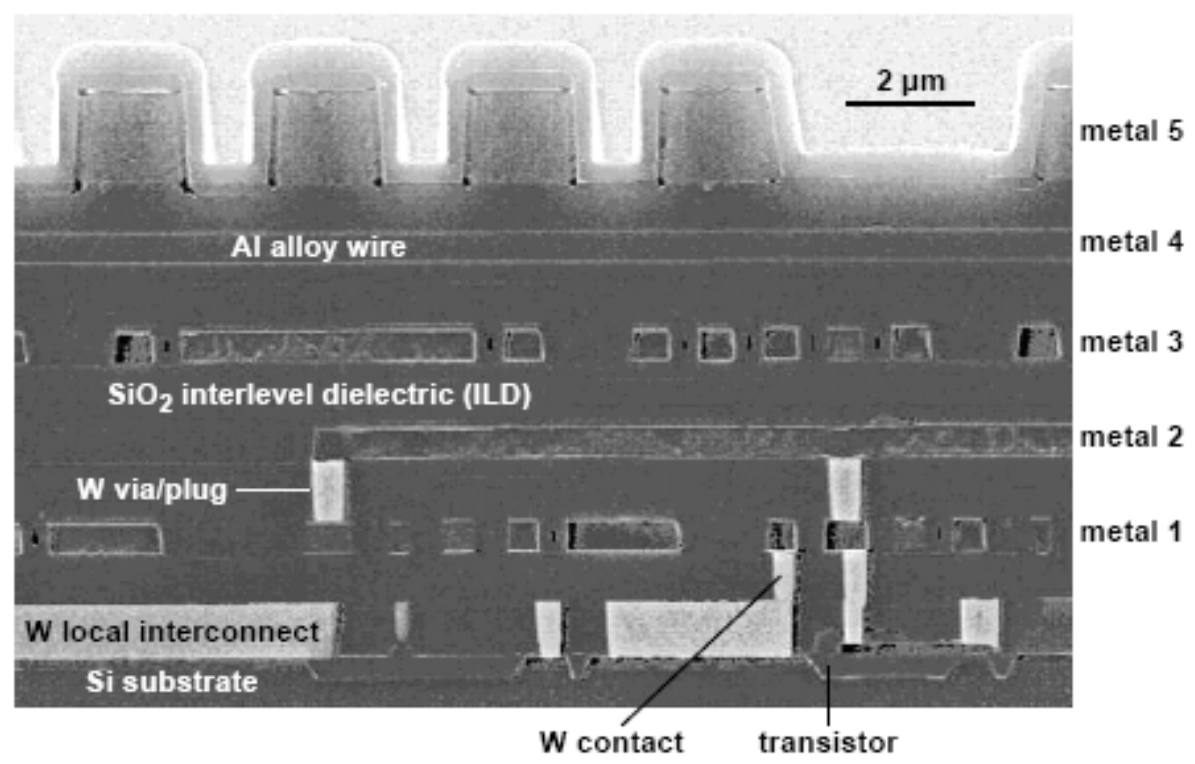

Figure 2.4 Cross-sectional scanning electron micrograph of state-of-the-art $0.25-\mu \mathrm{m}$ CMOS multilevel interconnect technology for high-performance logic ${ }^{32}$.

\subsubsection{Fabrication of Tungsten Vias}

The process sequence for forming tungsten vias is summarized in Figure $2.5^{33}$. First, a thick blanket oxide film of $\mathrm{SiO}_{2}$ is deposited on a planar surface, typically by PECVD (plasma-enhanced chemical vapor deposition) with a TEOS (Tetraethylorthosilicate) precursor at $350-400^{\circ} \mathrm{C}$. The oxide ILD is patterned by photoresist and then etched to expose the underlying metal layer typically a metal silicide. After the resist is stripped, the via opening is cleaned and then lined with a thin physical vapor deposition (PVD) of Ti layer. In modern CMOS technologies, PVD exclusively refers to sputtering. The Ti film serves as an adhesion layer and also decreases contact resistance to underlying conductors by reducing interfacial oxides. Titanium nitride is subsequently deposited in situ, either by sputtering or by CVD. Following that, the remaining part of the hole is conformally filled void-free with 
CVD tungsten by $\mathrm{SiH}_{4}$ reduction of $\mathrm{WF}_{6}$. The excess $\mathrm{W}, \mathrm{TiN}$, and $\mathrm{Ti}$ in the field regions are finally removed by chemical-mechanical polishing (CMP), This method of embedding metal structures in dielectrics is known as damascene process, paying tribute to an ancient art that originated in Damascus ${ }^{34}$.

Tungsten via technology has matured to the point where void-free and untapered vias with aggressive aspect ratios exceeding 3:1 are routinely formed, thus enabling increases in wiring density and reducing parasitic capacitance from under- and overlying wires. Advances in lithography alignment have also enabled borderless vias to be formed, thereby permitting even further improvements in wiring density. Damascene tungsten has been adapted as planar local interconnects for strapping source/drain and gate contacts ${ }^{35}$.

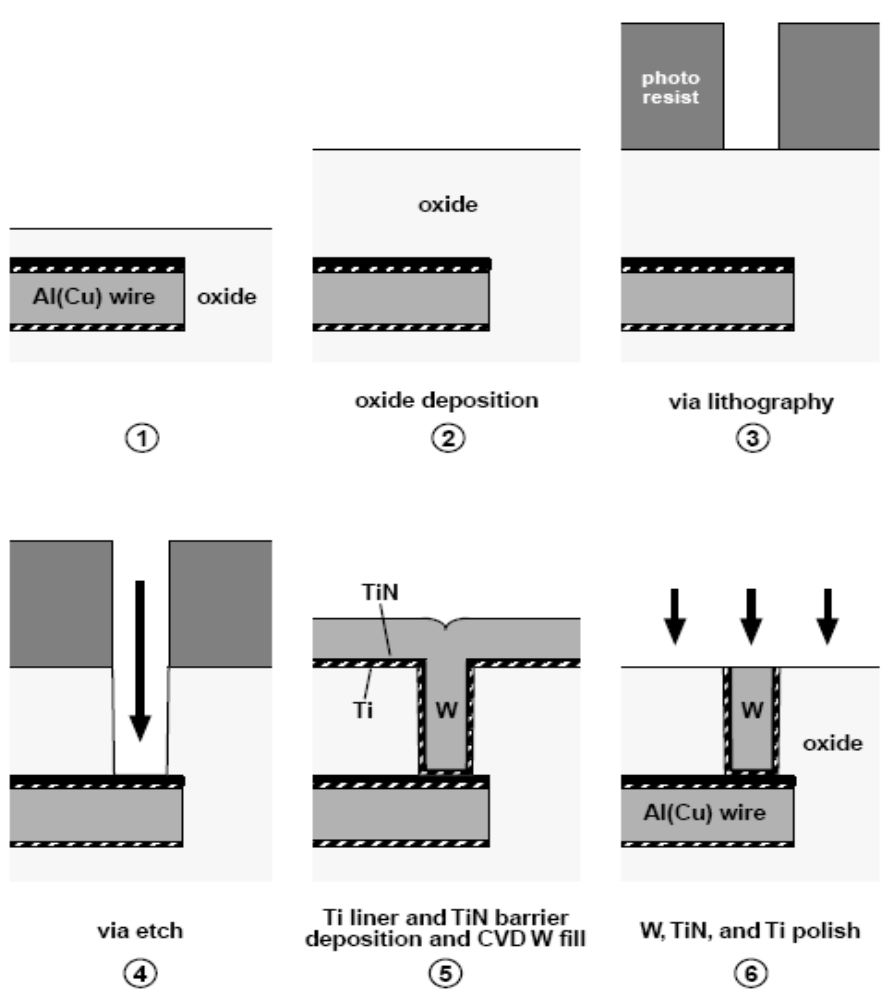

Figure 2.5 Process flow for fabrication of tungsten vias ${ }^{38}$. 
The main drawback of tungsten via technology is cost. Furthermore, processing of tungsten, a brittle refractory metal, is notorious for introducing particles and defects on the wafer which compromises yield. Therefore the development and employment of suitable and selective wet clean formulations to remove these defects is essential.

\subsubsection{Fabrication of Aluminum Alloy Wires}

The conventional process for forming Al alloy wires is summarized in Figure $2.6^{36}$. After via or contact CMP, metal is sputtered over a planarized surface. The metal deposition typically consists of a sequence of $\mathrm{Ti}, \mathrm{Al} / \mathrm{Cu}, \mathrm{Ti}$, and $\mathrm{TiN}$ depositions without breaking vacuum. The $\mathrm{Al}$ layer is alloyed with $0.5 \% \mathrm{Cu}$ which segregates to the $\mathrm{Al}$ grain boundaries for improved electromigration resistance ${ }^{37}$.

This “Ti-over-and-under” wiring uses Ti as a base layer for good adhesion, low contact resistance to underlying vias, and a seed for (111)-textured $\mathrm{Al} / \mathrm{Cu}$ grains which have better electromigration resistance. The $\mathrm{Al} / \mathrm{Cu}$ layer is sandwiched by thin $\mathrm{Ti}$ layers because subsequent thermal treatment forms $\mathrm{TiAl}_{3}$, a hard refractory intermetallic compound that further improves electromigration reliability as well as mechanical stability against stress-induced void and hillock formation. Finally, the reactively sputtered TiN film caps the metal stack to minimize the reflectivity of the stack and thus facilitate photolithographic fabrication of fine features. The process flow continues with the metal lithography and etch. After the metal is patterned, the photoresist is removed and the metal spaces are subsequently filled by a conformal oxide deposition. Void-free dielectric gap-fill remains an integration challenge, but 
can be achieved with high-density plasma (HDP) CVD oxide processes for $0.18-\mu \mathrm{m}$ technologies. The residual oxide topography is removed with oxide CMP, leaving a planar oxide surface as the starting point for fabricating the next level of vias.

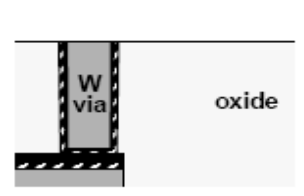

(1)

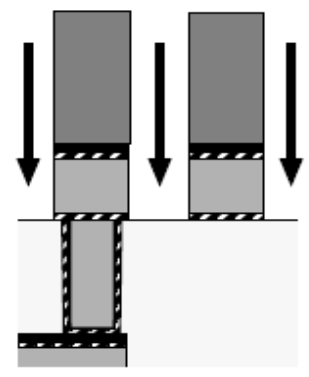

wire etch

(4)

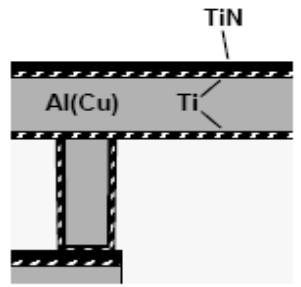

metal stack deposition

(2)

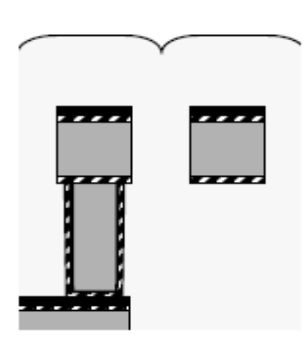

oxide gapfill deposition

(5)

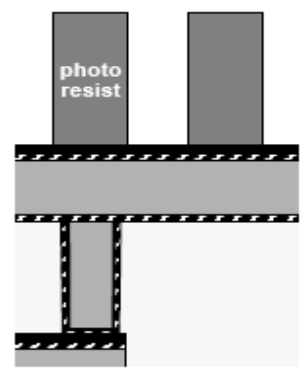

wire lithography

(3)

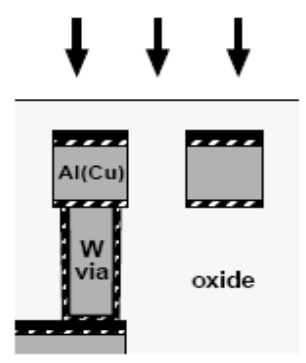

oxide polish

(6)

Figure 2.6 Process flow for fabrication of aluminum alloy wires ${ }^{41}$.

The interconnects in advanced logic IC's typically obey a hierarchical wiring scheme. The metal pitch (sum of line width and spacing) and thickness become progressively larger for interconnects further away from the transistors as shown in Table 2.1. The lower layers of interconnects are designed for maximum wiring density, while the uppermost layer(s) of thick interconnects are intended for long connections as well as power and ground distribution. 
Table 2.1 Interconnect Design Rules for $0.25-\mu \mathrm{m}$ Technology ${ }^{38}$

\begin{tabular}{|c|c|c|c|}
\hline Layer & Minimum Pitch & Thickness & Wire Aspect Ratio \\
\hline \hline metal 1 & $0.48 \mu \mathrm{m}$ & $0.48 \mu \mathrm{m}$ & $1.5: 1$ \\
\hline metal 2 & $0.93 \mu \mathrm{m}$ & $0.90 \mu \mathrm{m}$ & $1.9: 1$ \\
\hline metal 3 & $0.93 \mu \mathrm{m}$ & $0.90 \mu \mathrm{m}$ & $1.9: 1$ \\
\hline metal 4 & $1.60 \mu \mathrm{m}$ & $1.33 \mu \mathrm{m}$ & $1.7: 1$ \\
\hline metal 5 & $2.56 \mu \mathrm{m}$ & $1.90 \mu \mathrm{m}$ & $1.5: 1$ \\
\hline
\end{tabular}

\subsubsection{Dual-Damascene Copper Technology}

The cross-section of a manufacturable copper interconnect technology is shown in Figure 2.7. In this example, W local interconnects and contacts are fabricated first using the Damascene process described in Section 2.4.2. Then, six levels of $\mathrm{Cu}$ wiring are integrated with $\mathrm{Cu}$ vias between successive metal layers. Oxide is both the via- and wire-level dielectric. As mentioned in Chapter 1, the main technical issues with $\mathrm{Cu}$ integration are $\mathrm{Cu}$ line patterning and potential device contamination. Deep submicron copper interconnects cannot be formed using the conventional approach used in $\mathrm{Al}$ metallization. $\mathrm{Cu}$ halide compounds, e.g., chlorides and fluorides, that form during plasma etching are hardly volatile at low temperatures ${ }^{39}$, rendering the etch prohibitively slow. Unfortunately, photoresist cannot withstand the temperatures required for practical $\mathrm{Cu}$ etch rates $\left(>200^{\circ} \mathrm{C}\right)$. Dielectrics such as polyimide, oxide, and nitride have been explored as alternative masking materials but they complicate the lithography process. Wet etching and liftoff approaches have also been attempted ${ }^{40}$. However, line width control of deep submicron features is essentially impossible with these techniques. $\mathrm{Cu}$ is known to be 
a fast diffuser in silicon where it can act as a deep level acceptor in the silicon bandgap $^{41}$. Deep level states degrade minority carrier lifetime, causing high junction leakage in transistors and short retention times in DRAM's requiring faster refreshing which of course leads to increased power use. $\mathrm{Cu}$ also diffuses through silicon dioxide, especially under electrical bias ${ }^{42}$. These facts have raised serious concerns about device contamination should $\mathrm{Cu}$ be introduced into the backend of line processing. Successful implementation of $\mathrm{Cu}$ interconnects must consequently prevent any trace amounts of $\mathrm{Cu}$ from migrating to the Si substrate. This will not only involve added process complexity but also influence wafer handling and tool designs.

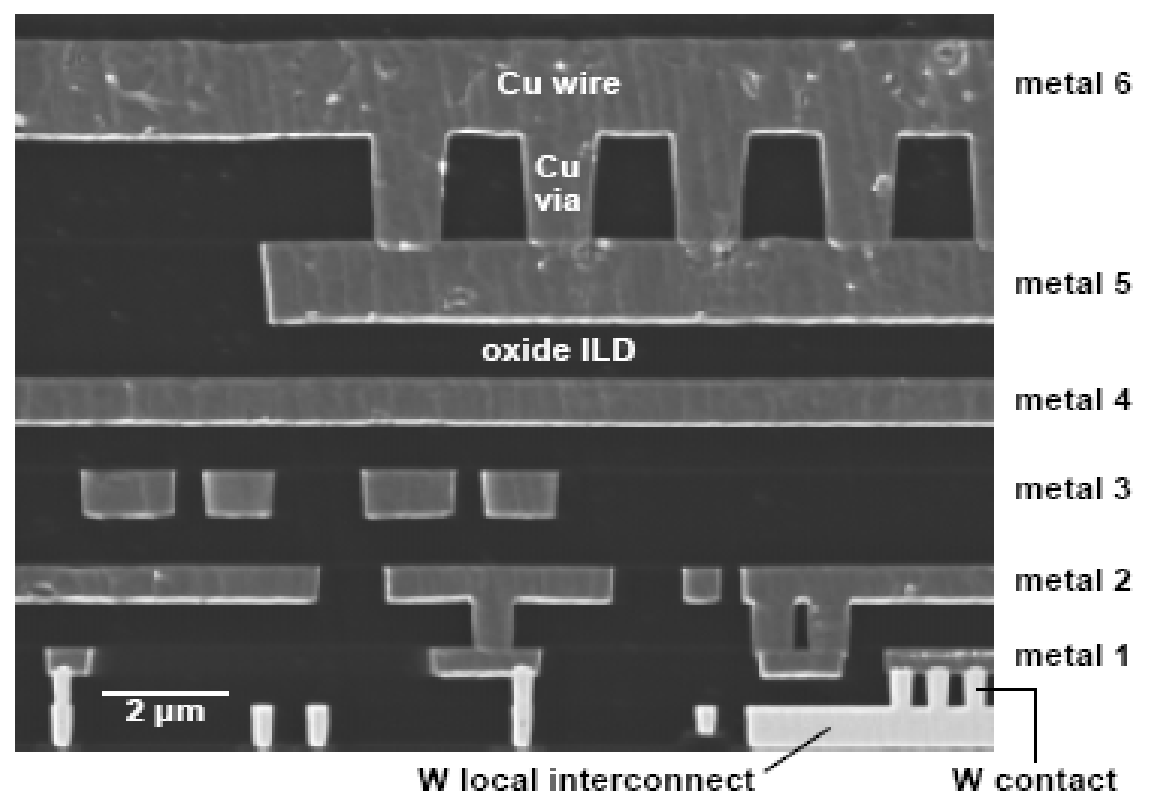

Figure 2.7 Scanning electron micrograph of manufacturable copper interconnect architecture demonstrated by $\mathrm{IBM}^{43}$.

The preceding obstacles are overcome by the dual-Damascene process developed at IBM with diffusion barriers surrounding the $\mathrm{Cu}$ interconnects ${ }^{44}$. Illustrated in Figure 2.8, dual-Damascene is a modified single-Damascene process 
where incorporating a second lithography step defines both wire trenches and via holes before they are backfilled with $\mathrm{Cu}$. Thus, $\mathrm{Cu}$ wires and vias are formed with only one metal fill and one CMP step. Otherwise, two complete single-Damascene flows will be required: one for the vias and the second for the overlying wires. This process simplification results in reduced cost and improved manufacturability. The $\mathrm{Cu}$ interconnects are isolated from the surrounding oxide by metal barrier materials on the interconnect side and bottom interfaces, and by a dielectric barrier above the interconnects. The individual steps in the dual-Damascene flow and the complex considerations in choosing barrier materials are elaborated in the subsections to follow.

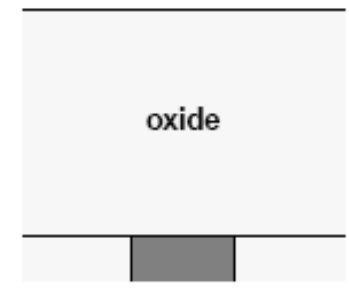

oxide deposition

(1)

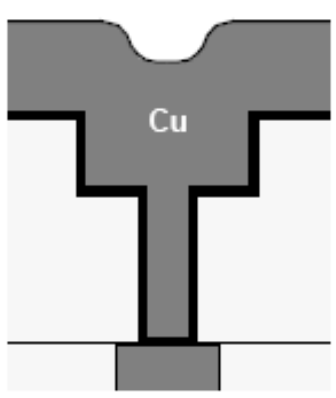

Cu fill

(4)

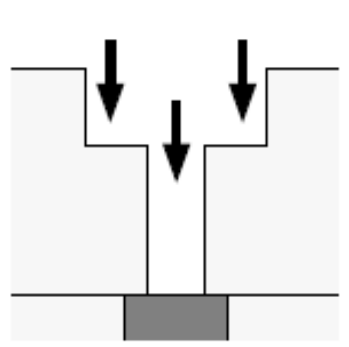

line and via etch

(2)

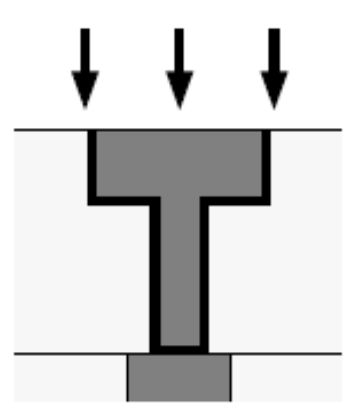

$\mathrm{Cu}$ and barrier polish

(5)

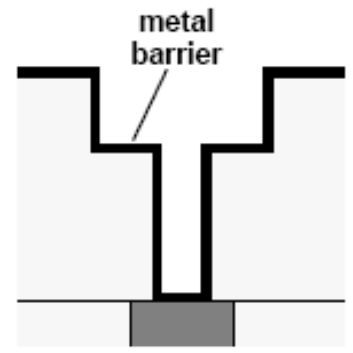

metal barrier deposition

(3)

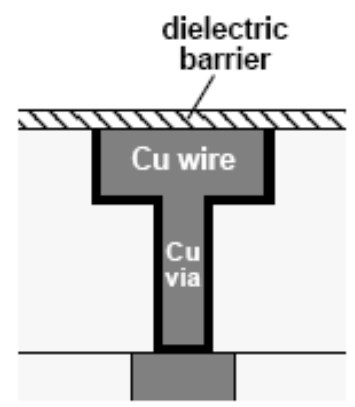

dielectric barrier passivation

(6)

Figure 2.8 Simplified dual-Damascene process flow for fabricating $\mathrm{Cu}$ interconnects ${ }^{49}$. 


\subsubsection{Dielectric Etch}

In a dual-Damascene flow, there are various methods of forming wire trenches and via holes to the underlying conductor ${ }^{45}$. Five approaches are summarized in Figure $2.9^{46,47,48,49,50}$. Their merits and issues are listed in Table 2.2. The specific flow that is ultimately implemented in manufacturing will vary from company to company and depends on the process strengths in lithography and etch within a corporation. 
(a) buried etch stop approach

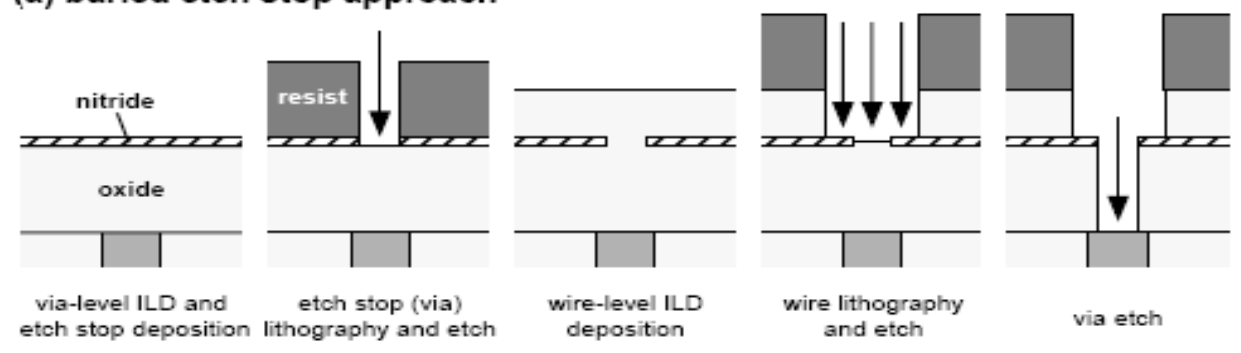

(b) clustered approach

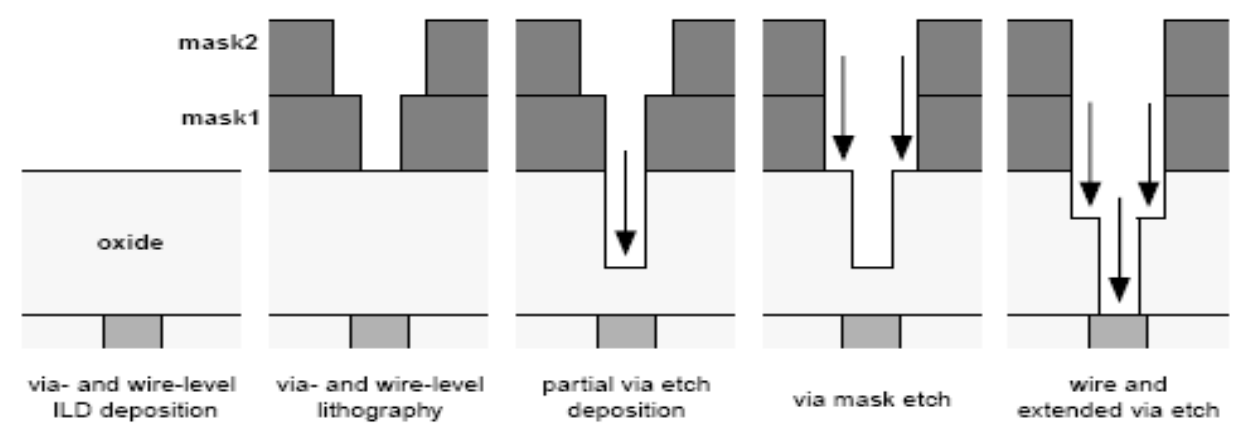

(c) partial via first approach

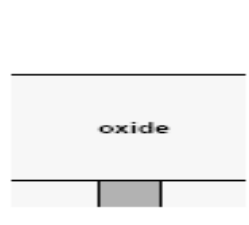

via-and wire-level

L deposition

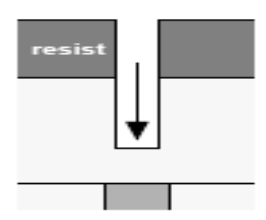

via lithography
and partial etch
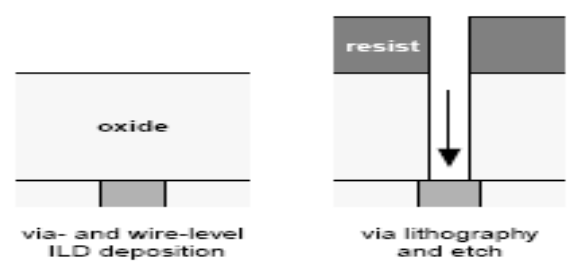

(e) line first approach

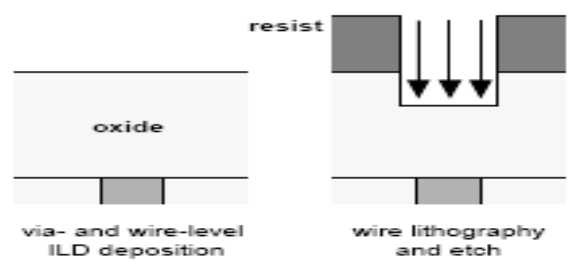

(d) full via first approach

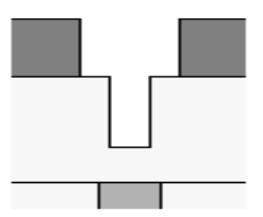

wire lithography

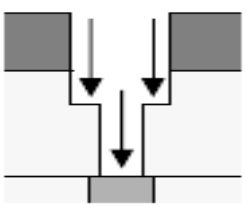
wire and
extended via etch

Figure 2.9 Dual-Damascene variations for defining wire trenches and via holes: (a) buried etch stop (b) clustered approaches (c) partial via first, (d) full via first, and (e) line first approaches. 
Table 2.2 Comparison of Dual-Damascene Dielectric Etch Approaches ${ }^{51}$.

\begin{tabular}{|c|c|c|}
\hline $\begin{array}{l}\text { Process } \\
\text { Flow }\end{array}$ & Advantages & Disadvantages \\
\hline $\begin{array}{l}\text { buried } \\
\text { etch stop }\end{array}$ & topography minimized & $\begin{array}{l}\text { etch process selectivity } \\
\text { and control are critical }\end{array}$ \\
\hline Clustered & process types grouped & $\begin{array}{l}\text { resist adhesion, } \\
\text { pattern transfer }\end{array}$ \\
\hline $\begin{array}{l}\text { partial } \\
\text { via first }\end{array}$ & $\begin{array}{l}\text { cleaner structure, } \\
\text { less critical etching }\end{array}$ & $\begin{array}{c}\text { lithography process difficulty } \\
\text { increased }\end{array}$ \\
\hline $\begin{array}{l}\text { full via } \\
\text { first }\end{array}$ & $\begin{array}{c}\text { lithography and etch processes } \\
\text { slightly easier; stacked via } \\
\text { trivial }\end{array}$ & $\begin{array}{l}\text { lithography rework and resist } \\
\text { cleaning process difficult }\end{array}$ \\
\hline line first & $\begin{array}{l}\text { easier etch process, } \\
\text { less topography for lithography }\end{array}$ & resist cleaning process critical \\
\hline
\end{tabular}

\subsubsection{Metal Barrier Deposition}

Barrier encapsulation of $\mathrm{Cu}$ interconnects is required to ensure that even trace levels of $\mathrm{Cu}$ do not diffuse through the surrounding dielectrics into the Si substrate. As illustrated in Figure 2.8, both metal and dielectric barriers will be needed for dualDamascene integration of $\mathrm{Cu}$ with oxide. Following the dielectric etch, the wire trenches and via holes must be lined with a conductive barrier material to clad the side and bottom boundaries of the $\mathrm{Cu}$ interconnects. Since barrier materials are generally very resistive compared to $\mathrm{Cu}$, barrier thickness must be kept to a minimum in order to preserve the effective conductivity advantage of $\mathrm{Cu}$ over $\mathrm{Al}$ alloys. Minimum barrier thicknesses in the 20-30 $\mathrm{nm}$ range are expected for $0.18-\mu \mathrm{m}$ technologies ${ }^{52}$. Besides possessing superior barrier property, metal barriers should additionally exhibit low contact resistance to $\mathrm{Cu}$. This requires an effective clean of the via holes following the dielectric etch. Since the via etch will expose underlying $\mathrm{Cu}$ wires, the cleaning 
solution must not corrode the copper, and consequently redeposit $\mathrm{Cu}$ from the solution onto the ILD surface and via hole sidewalls ${ }^{53}$ which is part of the focus of the strategies developed in this dissertation, chapter 3. The barrier layers should also have low stress and good adhesion to oxide. In addition, barriers play an important role in determining the microstructure of $\mathrm{Cu}$ films that are subsequently deposited. Similar to that of $\mathrm{Al}$ alloys, the electro-migration reliability of $\mathrm{Cu}$ interconnects depends on $\mathrm{Cu}$ film texture ${ }^{54}$. The texture and roughness of the barrier layer are only two factors affecting the texture that develops in $\mathrm{Cu}$ film deposition by electrochemical means ${ }^{55}$. Finally, for integration feasibility, it is important that metal barriers be deposited conformally into high aspect-ratio holes with low particle counts and must be easy to planarize $^{56}$

The above requirements have generated much interest to evaluate the barrier properties of refractory metals, primarily $\mathrm{Ti}, \mathrm{W}$, tantalum (Ta), and their nitrides ${ }^{57}$. With the wealth of experience gained from W via technology, the industry would ideally like to extend the use of $\mathrm{Ti} / \mathrm{TiN}$ liners in Damascene $\mathrm{Cu}$ integration. However, it appears that TiN may be inadequate as a barrier against $\mathrm{Cu}$ diffusion. Ta and $\mathrm{TaN}$ have shown great promise.

Amorphous materials are also being considered. Ternary films of TaSiN as thin as 5 nm have been shown to exhibit excellent barrier properties, presumably by removing fast $\mathrm{Cu}$ diffusion paths along the grain boundaries present in polycrystalline films ${ }^{58}$. These advanced barrier materials will draw more attention as barrier thickness scales with interconnect dimension. There exists a strong concurrent effort to develop 
deposition technologies capable of providing conformal coverage of barrier materials in very high aspect-ratio holes. Conformal coverage of the dielectric openings is essential because failure is expected to occur where the barrier is thinnest, usually at the lower corners and sidewalls of a via. Conventional dc magnetron sputtering cannot meet the stringent conformality requirements because the large angular distribution of sputtered atomic flux will result in more deposition along the top corners of the trenches before there is adequate barrier coverage along the via bottom and sidewalls. This casing will also increase the difficulty of the subsequent $\mathrm{Cu}$ fill. Figure 2.10 depicts Sputtering technology being modified to improve vertical flux directionality. Long-throw, collimated, and ionized metal plasma (IMP) sputtering technologies provide better but not completely conformal step coverage. Inherently a conformal process, CVD also has been actively investigated but is an expensive technology. The potential of CVD will depend on the extendibility of cheaper sputtering technologies for more aggressive geometries ${ }^{59}$.

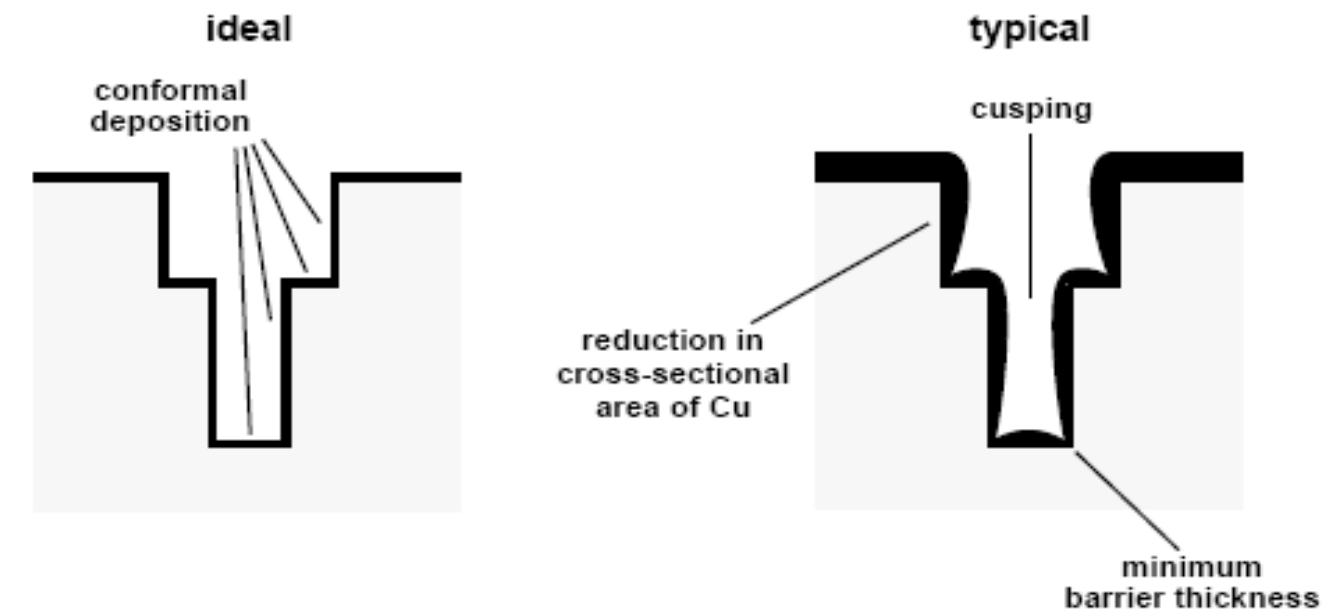

Figure 2.10 Comparison of ideal and typical step coverage of a metal barrier deposited by PVD $^{60}$. 
The development of a metal barrier technology is key to successful integration of $\mathrm{Cu}$. Although many implementation details remain undisclosed by companies involved, there is growing consensus in the industry to employ Ta liners and TaN barriers deposited by IMP sputtering.

\subsubsection{Copper Deposition}

After metal barrier deposition, the trenches and vias are filled with $\mathrm{Cu}$. Many technologies have been explored to identify a cost-effective solution capable of high aspect ratio and void-free $\mathrm{Cu}$ fill. Four are described in this discussion: PVD, CVD, electroless plating, and electroplating. PVD techniques, even with improved flux directionality, are incapable of achieving void-free $\mathrm{Cu}$ fill. Revisiting Figure 2.10, the cusping that develops during sputtering will eventually pinch off the $\mathrm{Cu}$ film near the top of the trench and form a keyhole. However, good trench filling has been

demonstrated through reflow after sputtering ${ }^{61,62}$. First, the trench is partially filled by sputtering. In a subsequent in situ heat treatment, typically at $450^{\circ} \mathrm{C}$ for 30 minutes, the metal atoms redistribute from the field region into the trench, thereby completing the fill. The reflow process is thermodynamically driven by surface diffusion which minimizes the surface energy of the $\mathrm{Cu}$ film. It is very sensitive to the purity of the ambient gas during anneal, microstructural inhomogeneities in the $\mathrm{Cu}$ film, the wetability of the barrier underlayers, and the density of trench features. Moreover, the relatively high thermal budget incurred by the reflow anneal may unnecessarily impose stricter barrier requirements. The limited process latitude renders sputter reflow inadequate for manufacturing. 
Due to its superior step coverage over PVD, CVD has naturally received much attention. CVD Cu films are deposited by thermal decomposition of organometallic (OMCVD) precursors at 150 to $200^{\circ} \mathrm{C}^{63}$. The most extensively investigated precursor is $\mathrm{Cu}(\mathrm{hfac})(\mathrm{tmvs})$, abbreviated for copper (I) hexafluoroacetylacetonate trimethylvinylsilane. Although excellent trench fill in aggressive geometries have been demonstrated, the main bottleneck preventing widespread use of CVD Cu is cost. The price of the $\mathrm{Cu}$ precursor will remain prohibitively high until cheaper alternative fill technologies can no longer accommodate the fill requirements as interconnects continue to scale.

Electroless plating, a cheap and simple means of selectively depositing thin $\mathrm{Cu}$ films, was also considered ${ }^{64}$. Wafers are immersed in a heated bath of aqueous $\mathrm{Cu}^{2+}$ ions. $\mathrm{Cu}$ atoms are then supplied to the wafer surface by catalytic reduction of the $\mathrm{Cu}^{2+}$ ions, but only at exposed conductive surfaces of the wafer. Electroless plating was a serious contender during the early stages of $\mathrm{Cu}$ process development. Unfortunately, its primary drawback is lack of process control during deposition. Deposition will proceed spontaneously and depend primarily on the plating solution chemistry and the seed layer. Moreover, the microstructure of electroless $\mathrm{Cu}$ films generally consists of very fine grains, implying poor electromigration reliability. For these reasons, electroless $\mathrm{Cu}$ is not considered feasible for production.

Electroplating emerged as the most promising and cost-effective $\mathrm{Cu}$ deposition technology, ${ }^{65}$ having already been demonstrated for manufacturability ${ }^{66}$. 
In electrochemical deposition of copper, the wafer is coated with a thin seed layer of $\mathrm{Cu}$, typically by sputtering, and immersed in a solution containing $\mathrm{Cu}^{2+}$ ions. Although the wafer will have already been lined with a conductive barrier and a thin $\mathrm{Cu}$ seed layer, the $\mathrm{Cu}$ seed layer is needed because electroplating may not occur on some barriers. Electrical contact is made to the seed layer which serves as the cathode. An electrical current is supplied to the cathode to reduce $\mathrm{Cu}$ ions at the wafer, thereby depositing atomic $\mathrm{Cu}$ on the $\mathrm{Cu}$ seed. As $\mathrm{Cu}$ ions are plated out of the solution onto the wafer, the $\mathrm{Cu}$ anode simultaneously undergoes oxidation to replenish the supply of $\mathrm{Cu}$ ions in the solution. See Figure 2.11.

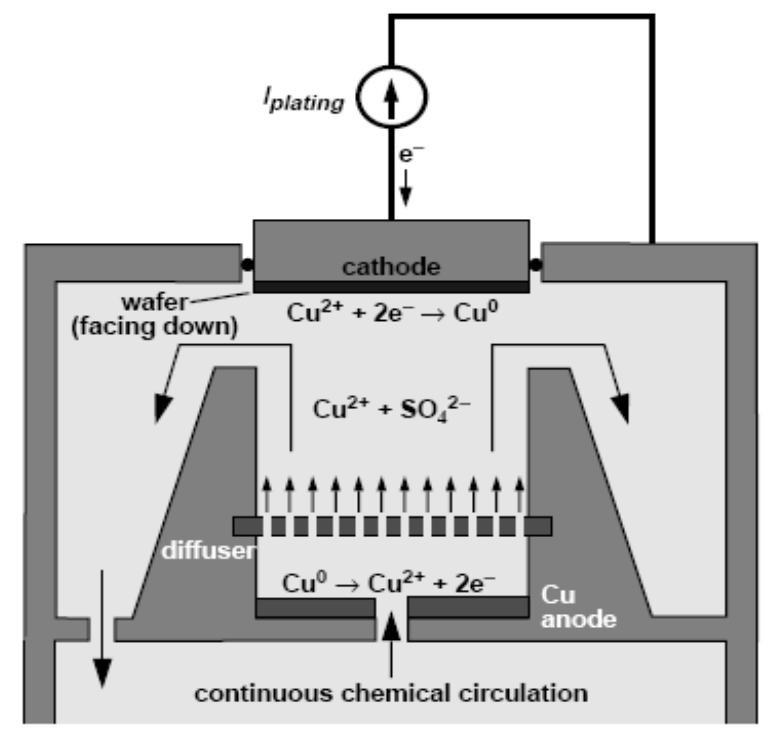

Figure 2.11 Schematic of a $\mathrm{Cu}$ electroplating system $)^{71}$.

In principle a relatively simple technology, $\mathrm{Cu}$ electroplating in practice is fairly complex. A manufacturable process must demonstrate good fill capability, step coverage, film morphology, across-wafer and wafer-to-wafer uniformities, and practical deposition rates. To achieve void-free fill, the contents of the electroplating solution and the way in which the electrical current is applied must be optimized. 
Otherwise, keyholes may form in the trench since the plating rate is higher at the trench shoulders, where the current density is highest, than at the trench bottom. The plating bath primarily consists of aqueous copper sulfate $\left(\mathrm{CuSO}_{4}\right)$ and sulfuric acid $\left(\mathrm{H}_{2} \mathrm{SO}_{4}\right)$ but also contains trace quantities of organic additives (e.g., thiourea, disulfides, and polyamines). These additives improve the quality of the deposited $\mathrm{Cu}$ film by, for example, enhancing deposition at the bottom of trenches, serving as wetting agents for good film nucleation, and relieving deposited film stress ${ }^{67}$. The trench and via filling capability of electroplating is also improved by modulating the magnitude and direction of the electrical current. Reversing the polarity of the applied current causes oxidation or etching of $\mathrm{Cu}$ to occur at the wafer surface. Since the etching rate is also a direct function of current density, a deposition/etch sequence is employed to remove copper from the trench shoulder more quickly than from the trench bottom during the etch cycle, resulting in more conformal coverage. With both pulsed plating waveform and bath chemistry optimization, high aspect-ratio trenches and vias can be successfully filled ${ }^{68}$. Given the appropriate barrier and $\mathrm{Cu}$ seed layers and microstructures, plated $\mathrm{Cu}$ films with large grain sizes and a near-bamboo microstructure can be obtained. These factors are believed to be responsible for the good electromigration resistance of plated $\mathrm{Cu}^{69}$.

\subsubsection{Chemical-Mechanical Polishing}

After the trenches and vias are filled with $\mathrm{Cu}$, the excess $\mathrm{Cu}$ in the field region is removed by chemical-mechanical polishing (CMP). Pioneered by IBM, CMP is unquestionably the key enabling technology in Damascene integration ${ }^{70}$. Figure 2.12 
illustrates a typical CMP system. Both chemical reactivity and mechanical abrasion play important roles in the selective removal of a film from the wafer surface. Chemicals in the slurry react with the film surface, typically forming a thin oxidized layer. This layer is subsequently removed by mechanical abrasion due to fine particles in the slurry under the pressure of the polishing pad. The wafer surface becomes progressively planar with polishing time since the removal or polishing rate increases with local pad pressure.

In metal CMP, a good balance must exist between chemical and mechanical components to achieve optimum planarization. If the mechanical component is too dominant, surface scratches and nonuniform polishing may result. On the other hand, if the chemical component is too dominant, overpolishing can result in severe surface topography due to the selectivity of the slurry chemistry against dielectric removal. Mechanical abrasion depends on the size and concentration of slurry particles, hardness and surface roughness of the pad, pad pressure, and the rotational speeds of the pad and wafer. The chemical component is controlled by the chemistry, concentration, and $\mathrm{pH}$ of the slurry. The CMP process must also minimize pattern density and feature size effects in order to avoid dielectric erosion and metal dishing. 


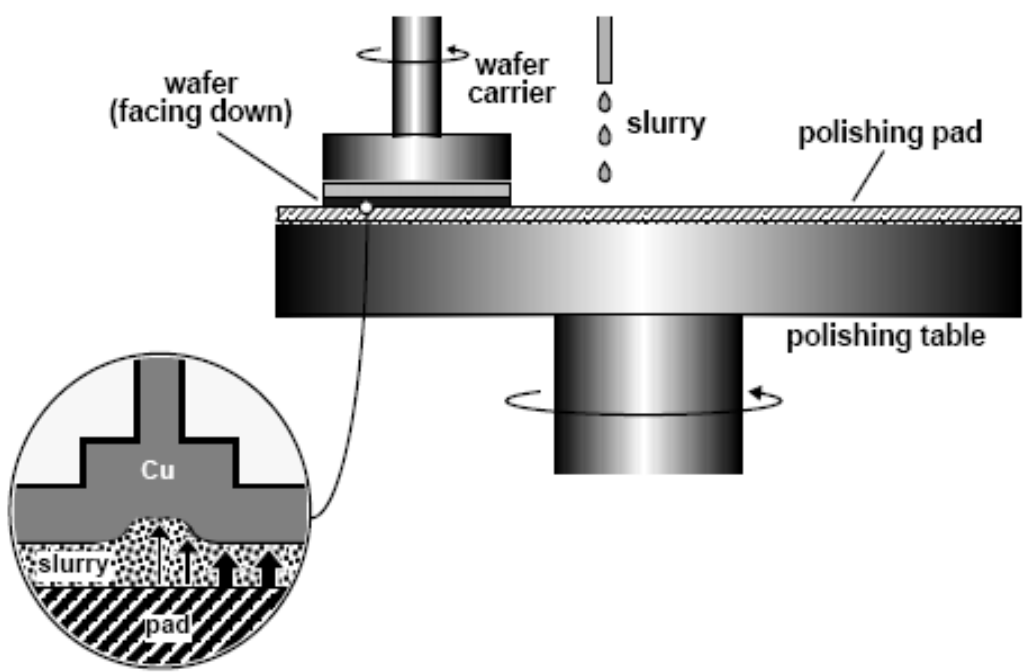

Figure 2.12 Schematic of a typical chemical-mechanical polishing system ${ }^{76}$.

Compared to W CMP, there are several additional challenges unique to $\mathrm{Cu}$ $\mathrm{CMP}^{71}$. Unlike $\mathrm{W}, \mathrm{Cu}$ is a relatively soft metal which is easily corroded and is prone to scratches and embedded particles. In addition, $\mathrm{Cu} \mathrm{CMP}$ is complicated by the underlying conductive barrier layers which must also be removed. Like any CMP process, the post CMP clean is critical in removing traces of slurry from the polished surface. However, since $\mathrm{Cu}$ CMP is inherently a wet process that will liberate $\mathrm{Cu}^{2+}$ by-products, the post-CMP cleaning solution has the additional burden of removing these ions from the wafer surface in order to minimize the potential of device contamination.

\subsubsection{Dielectric Barrier Passivation}

Following $\mathrm{Cu} \mathrm{CMP}$, the $\mathrm{Cu}$ interconnects must be capped by a dielectric barrier (also known as Etch Stop (ES) layer) such as PECVD silicon nitride or silicon carbide. The nitride passivation completes the fabrication of one level of $\mathrm{Cu}$ wires and vias. 


\subsection{Interconnects reliability and limitations}

\subsubsection{Limitations with Interconnect Scaling}

IC performance is dictated by the delays arising from propagation of electrical signals through the circuit. These delays have traditionally been associated with transistor switching. Performance improvements have thus been achieved primarily through reductions in the size of transistor geometries, most notably the gate length in MOS technology ${ }^{72}$. With this paradigm, the industry has enjoyed continual improvements in IC performance until only recent years. The scaling of transistors has necessitated an increase in wiring density to accommodate increasing transistor

densities over larger chip areas ${ }^{73}$. This has been made possible by advances in interconnect technology enabling continued miniaturization of interconnects as well as stacking of additional levels of metallization.

\subsubsection{Interconnect Delays}

Although signals propagate faster through transistors as dimensions are scaled down, propagation through interconnects unfortunately becomes slower. In fact, in submicron logic technologies, interconnects have become the performance limiter (Figure 2.13) ${ }^{74}$. Moreover, the increasing complexity of multilevel wiring architectures has escalated the cost of interconnect manufacturing to comprise over half of the total wafer processing $\operatorname{cost}^{75}$. 


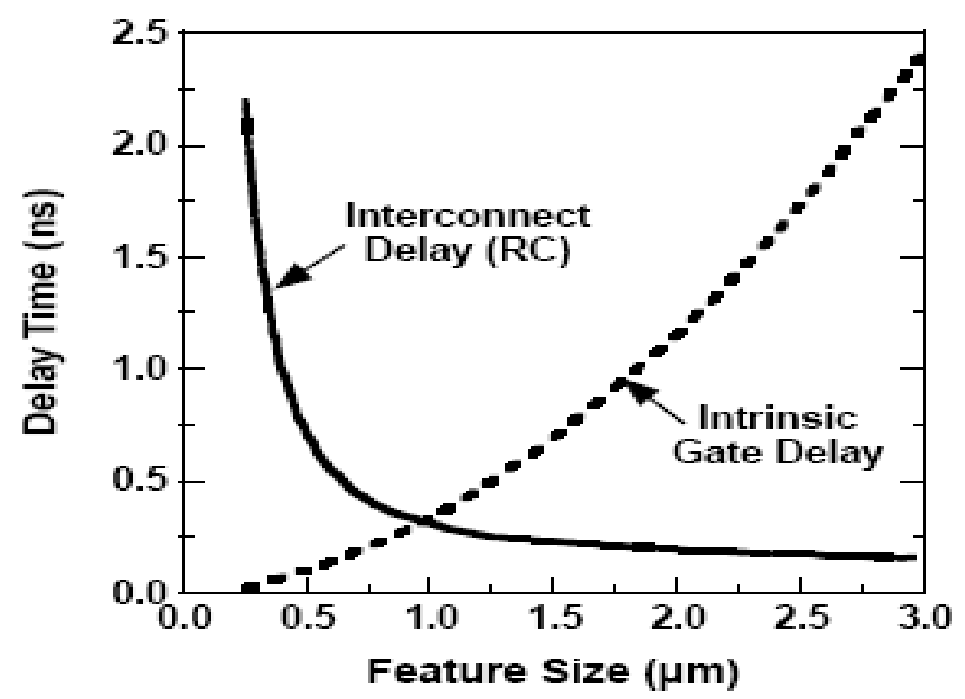

Figure 2.13 Intrinsic gate and interconnect delays as a function of minimum feature $\operatorname{size}^{80}$.

The interconnect delay can be estimated by the $R C$ delay - the product of interconnect line resistance, $R$, and the parasitic capacitance coupling the interconnect to adjacent lines and underlying Si substrate. The RC delay is given by

$$
R C \approx \rho \varepsilon \frac{L^{2}}{t_{M} t_{I L D}}
$$

Where $\rho, L$, and $t_{M}$, are respectively the resistivity, length, and thickness of the interconnect, while $\varepsilon$ and $t_{I L D}$ are the permittivity and thickness of the ILD respectively. For convenience, $\varepsilon$ is usually cited in terms of the dielectric constant, $\kappa$, defined as $\varepsilon / \varepsilon_{0}$ where $\varepsilon_{0}$ is the permittivity of free space.

Various options exist to reduce $R C$ delays. In some cases, the length of an interconnect in a critical path can be reduced if additional levels of wiring were available $^{76}$. However, this solution provides little benefit to designs already optimized 
for maximum wiring and transistor densities. Moreover, extending any process flow will invariably increase cost and reduce chip yield. The $R C$ delay can also be reduced by dividing long interconnects into shorter segments and inserting repeaters (inverter stages) between consecutive segments ${ }^{77}$. Repeaters sharpen slowly rising and falling transitions of a logic stage output, hence shortening the time required to trigger the input of subsequent logic stages. Repeaters are effective provided they can significantly reduce the effective $R C$ load driven by the preceding stage. The penalty though is increased power consumption, chip area, and design cost. Yet another attempt to minimize interconnect delay is to lower the IC operating temperature where both interconnect propagation and device switching are faster ${ }^{78}$. Unfortunately, the added complexity and cost of cooling the chip can only be justified in specialized applications where performance is the only objective. The technological solution for $R C$ delay reduction is to replace the existing conductors and insulators that comprise the interconnect system with lower $\rho$ and $\kappa$ materials. The conventional architecture consisted of aluminum (Al) alloy conductors $(\rho \approx 3.0-3.3 \mu \Omega$-cm) isolated by silicon dioxide $(\mathrm{SiO} 2)$ or oxide $(\kappa=4.0-4.5)$. The different layers are connected by tungsten (W) vias $(\rho \approx 5.7 \mu \Omega$-cm).

\subsubsection{Reliability}

Interconnect reliability is also compromised by scaling ${ }^{79,80,81}$. Copper interconnects suffer from failure modes associated with the manufacturing process and integration schemes of DD Cu structures. A common failure mechanism is linked to 
the challenge of providing void free lines and vias completely encapsulated with continuous liners which are common places for early reliability failures.

In this section, two key reliability areas, electromigration and stress-induced voiding (SIV) are briefly discussed. Electromigration is one of the primary reliability failure mechanisms in interconnects. Since supply voltages are not reduced as aggressively as the interconnect dimensions, interconnects must support higher current densities and are consequently prone to earlier electromigration failure. Electromigration is thermally activated atomic diffusion induced by an electron current. At current densities as high as those used in IC's, there is enough transfer of momentum from the electrons to the atoms of the conductor to cause a net atomic selfdiffusion in the direction of electron transport. This mass movement changes the atomic density along the interconnect and consequently builds up mechanical stresses. Tensile stresses in the line eventually lead to formation of voids that ultimately cause open-circuit failures while compressive stresses lead to formation of hillocks that protrude through the encapsulating ILD and cause short-circuit failures with adjacent interconnects or interconnects in a different level. There are two competing forces governing electromigration which can be summarized by he following drift equation ${ }^{82}$ :

$$
J=\left(\frac{D_{\mathrm{eff}} C}{k T}\right)\left(Z_{\mathrm{eff}}^{*} e \rho j-\Omega \frac{\partial \sigma}{\partial x}\right)
$$

The electron wind force is proportional to the effective charge number $Z^{*}$ eff, the electronic charge e , metal resistivity $\rho$, and the current density $j$. The second term in (2-2) is the back stress gradient generated by the surrounding dielectrics and barrier 
layers $^{83}$ to counter balance the electron wind much like the confinement of a glass tube to a liquid column under gravity. Here, $\Omega$ is the atomic volume and $(\partial \sigma / \partial \chi)$ is the stress gradient along the interconnect line. The effective diffusivity $\mathrm{D}_{\text {eff }}$ includes contributions from all diffusion paths and each could have a different $Z^{*}$ because the wind force varies according to the local environment surrounding a given atom ${ }^{84}$.

Pure aluminum interconnects exhibit poor resistance against electromigration. To alleviate this deficiency, the industry has invested exhaustive efforts to improve the reliability of aluminum metallization, incorporating optimized microstructures, alloying with impurities, and cladding the interconnect with hard liner materials ${ }^{85}$. These sophistications have markedly prolonged the lifetime of Al interconnects to the point that failures are concentrated to the intermetallic interfaces between the $\mathrm{Al}$ wires and $\mathrm{W}$ vias.

\subsubsection{Capacitance Issues}

With minimum interconnect feature sizes in the $100 \mathrm{~nm}$ regime, interconnect capacitance increases from continued scaling because of dominating line-to-line coupling between adjacent wires of the same metal level as shown in Figure 2.14. Besides the RC delay, capacitive parasitics also present other limitations on IC performance, dynamic power dissipation and crosstalk noise ${ }^{86,87,88,89}$. Continued scaling of interconnects will only intensify these issues. 

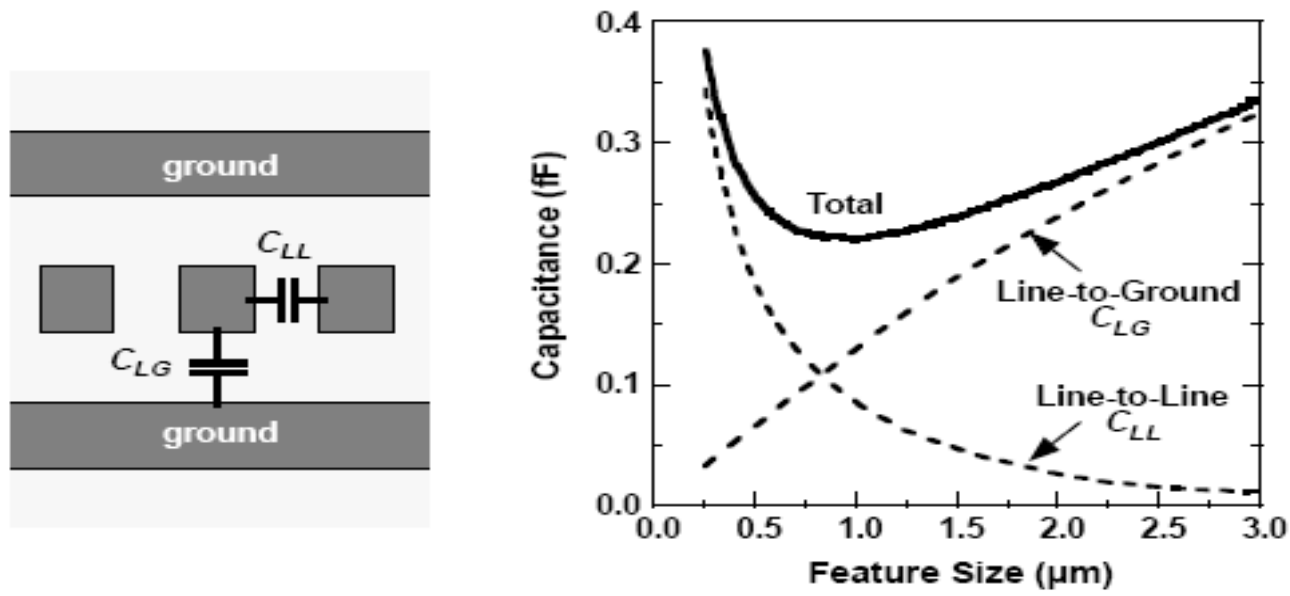

Figure 2.14 Impact of line-to-ground and line-to-line coupling on total interconnect capacitance $^{90}$.

With increasing device densities and operating frequencies, the density of power that is generated on-chip also increases. See Figure 2.15. Chips today operate at $85-120^{\circ} \mathrm{C}$ due to device and interconnect heating. This issue imposes increasing demands on already expensive packaging solutions to transport heat away from the chip in order to prevent quiescent temperatures from escalating even further. Otherwise, chip performance and reliability will degrade.

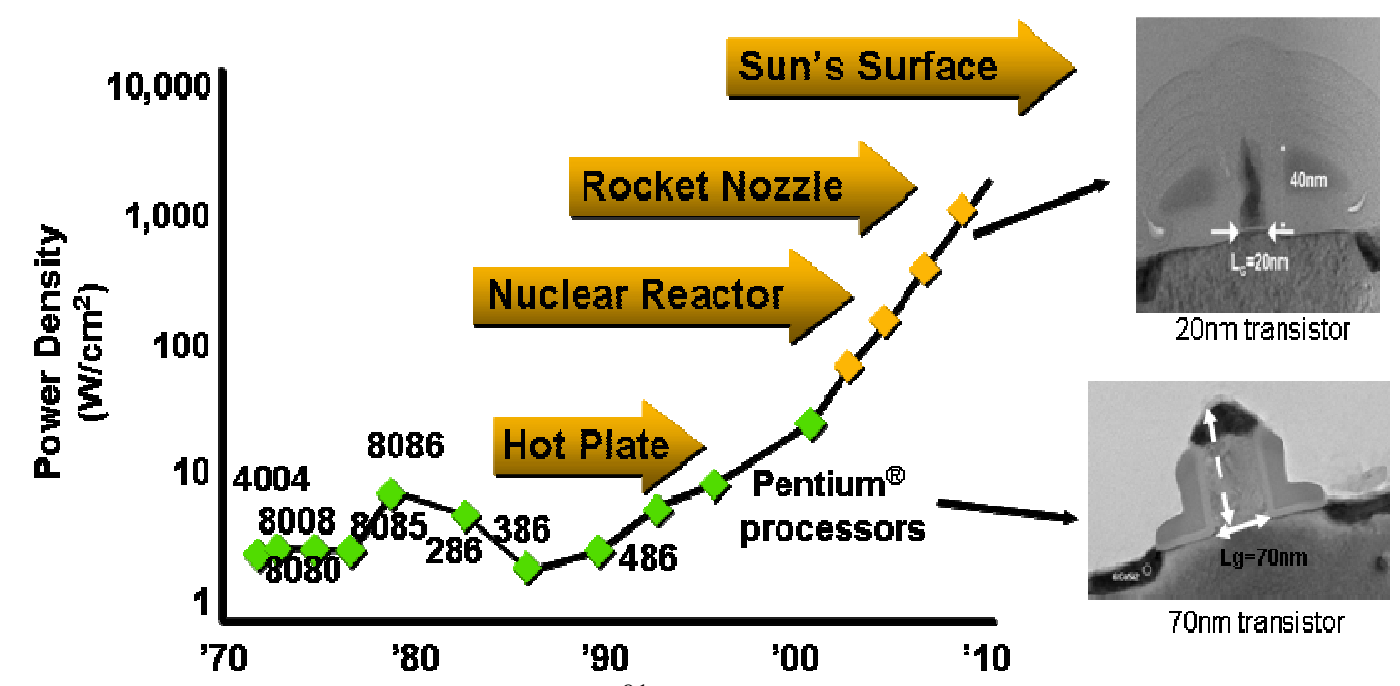

Figure 2.15 Power density problem ${ }^{91}$. 
On-chip power dissipation consists of both static and dynamic components. In MOS technology, static power dissipation is primarily due to transistor junction leakage. Dynamic power is dissipated during the switching transients in digital circuits and is estimated by

$$
P_{\text {dynamic }}=1 / 2 \alpha C V^{2} f
$$

Where $C$ is the capacitive load at a circuit node, $V$ is the power supply voltage, $f$ is the clock frequency, and $\alpha$ represents the probability that the node transitions in a given clock cycle. This shows that lower $\kappa$ ILD's have a direct impact on dynamic power reduction. Crosstalk noise is another undesirable consequence of interconnect scaling, most seriously affecting wires of minimum spacing. A propagating voltage transient in an interconnect will capacitively couple voltage transients into adjacent interconnects; the smaller the metal line separation, the larger the crosstalk peak voltage that is induced ${ }^{92}$. Therefore, for circuits to be resilient to crosstalk, supply voltages cannot be lowered too aggressively in order to maintain adequate noise margins. Lateral coupling can be minimized by manipulating the geometries (aspect ratios) of the lines and spaces, but altering aspect ratios only trades line-to-line for line-to-ground capacitance ${ }^{93}$.

\subsection{Problem significance}

More new materials are being introduced today than at any other time in the history of ULSI fabrication (Braun, 2001); In BEOL new low dielectric constant materials are replacing $\mathrm{SiO}_{2}$ as the insulator and copper as discussed before has 
replaced aluminum as the conductor. The dual damascene technique is the primary means for building interconnects using copper ${ }^{94}$.

A specific challenge is the selective wet etching of sacrificial thin layers and the cleaning of post dry etch residues, especially in the vias, which are small holes patterned in an interlayer dielectric to connect two metal layers. These features may prove most difficult to clean due to the high-aspect ratio and reduced feature size (sub$100 \mathrm{~nm}$ ) demanded in future generations of devices, as defined in the International Technology Roadmap for Semiconductors, 2001 (Table 2.3). The structures that remain after etching vias are patterned layers that may contain numerous post-etch residues. The mechanisms by which the residues form include: (1) The formation of nonvolatile products in secondary reactions between gas phase neutrals and the film to be etched; (2) Sputtering of the etch mask resulting in redeposition on via sidewalls and bottom, and (3) Back-sputtering of the underlying films once exposed during over etch.

Table 2.3 Wiring pitch and aspect ratio are the geometries of interest for cleaning Semiconductor Industry Association (SIA), 2001.

\begin{tabular}{ccccc}
\hline \hline & $\mathbf{1 9 9 9}$ & $\mathbf{2 0 0 2}$ & $\mathbf{2 0 0 5}$ & $\mathbf{2 0 0 8}$ \\
\hline $\begin{array}{c}\text { Local wiring pitch (nm) } \\
\text { Local wiring aspect } \\
\quad \text { ratio }\end{array}$ & 500 & 365 & 265 & 185 \\
\hline
\end{tabular}

Residues at the bottom, sidewalls, and top of these features can cause poor contact upon subsequent depositions, may alter the properties of low-k dielectric films, and may lower the conductance of metal lines. Inadequate cleans show 1000 times greater dielectric leakage current and a wider capacitance range ${ }^{95}$. Therefore, these 
structures must be free of essentially all residues. The residue that remains after an etching process is dependent upon the dielectric material, the etching gas, and all other exposed materials. Some of the etch residues that have been characterized and reported ${ }^{96}$ are, $\mathrm{CF}_{\mathrm{x}}-\mathrm{Cu}, \mathrm{CH}_{\mathrm{x}} \mathrm{F}_{\mathrm{y}}, \mathrm{CuF}_{\mathrm{x}}, \mathrm{CuO}_{\mathrm{x}}$ for $\mathrm{SiO}_{2}$ based dielectrics with or without dopants such as $\mathrm{C}, \mathrm{F}, \mathrm{N}$, and $\mathrm{CuO}_{\mathrm{x}}, \mathrm{CH}_{\mathrm{x}}$ for Carbon based low-k dielectric polymers.

The primary chemical formulations for BEOL cleaning involve corrosive solvents such a hydroxylamine ${ }^{97}$ and other corrosives/carcinogens that are not always compatible with $\mathrm{W}, \mathrm{Cu}$, and dielectric materials. Non-corrosive materials such as fluoride-based chemistries have been suggested as alternatives ${ }^{98}$. Yet these often result in etching of exposed silicon dioxide. The hydroxylamine- and fluoride-based formulations often contain a mixture of organic solvents and water. Current commercial formulations have proven to be non-compatible, non selective to stripping metallic HMs such as Ti, and ineffective in removing dry etch residues ${ }^{99}$. Unless improved and highly selective wet etch/clean solutions are developed, high-yield manufacturing will not be possible for next generation devices. 


\section{CHAPTER 3}

\section{Corrosion Behavior of Copper Thin Films in Organic \\ HF containing cleaning solution}

\subsection{Introduction}

In advanced interconnect systems of today, copper is the choice of metallization for Ultra Large Scale Integration (ULSI). Copper wiring is now employed in all interconnects layers with up to 12 metallization levels in advanced microprocessors. In principle, interconnects are electrical paths or charge carriers made out of metal lines and are separated by insulating interlayer dielectric (ILD) material. The replacement of aluminum alloys by copper mandated prominent changes in integration, metallization, and patterning process technologies. For instance, the introduction of copper in semiconductor devices has brought attention to the phenomena of thin film corrosion that must be circumvented for optimal device performance, reliability, and longevity. This has also mandated the adoption of wet etch clean chemistries that are $\mathrm{Cu}$-compatible for the integration of dual damascene (DD) patterning of copper interconnects ${ }^{100}$. A simple two layer DD interconnect system is illustrated in Figure 3.1. In such a system, the electrical signal that allows transistors to communicate with one another and with the outside world is transmitted through the metal lines within any given metallization level and through copper filled vias from one metallization level to another. In DD integration, the via resistance is mainly determined by the via diameter and thickness, resistivity of the copper 
diffusion barrier being used and by the overall interfacial resistance with the underlying metal layer. Etch residues resulting from plasma etch during patterning process steps may give rise to high via resistance or even create opens.

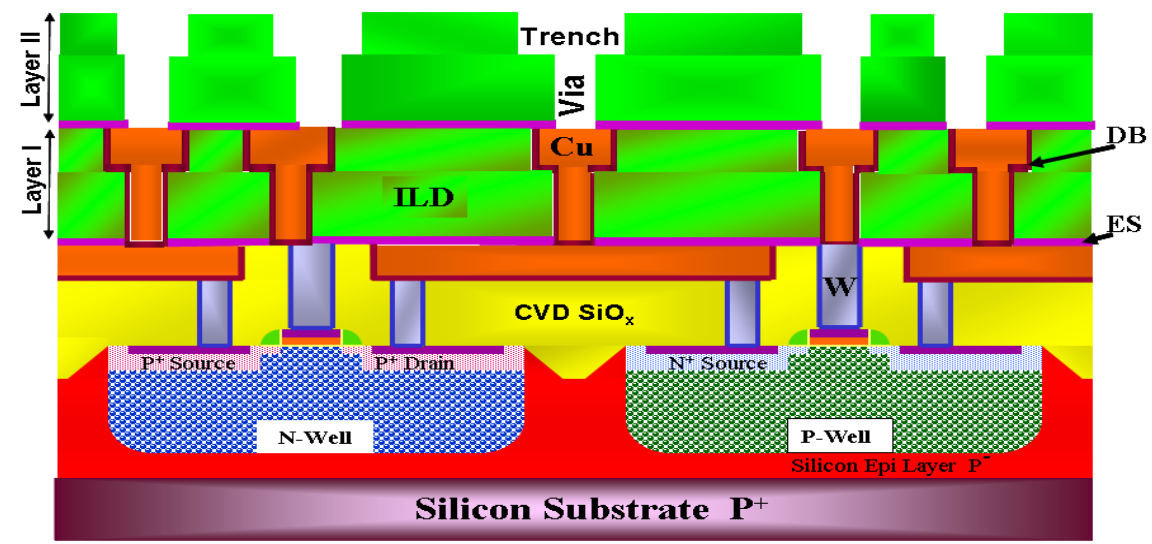

Figure 3.1 A simple two layers DD interconnect system (not to scale) showing various components such as Diffusion Barrier (DB), Etch Stop (ES) layer, and ILD.

There have been many approaches explored to remove the plasma etch residues. These include the use of $\mathrm{O}_{2}, \mathrm{NF}_{3} / \mathrm{Ar}$ and $\mathrm{He} / \mathrm{H}_{2}$ plasma chemistries to clean polymeric residues ${ }^{101,102,103,104,105}$, as well as the use of wet etch chemistries such as aqueous dilute $\mathrm{HF}^{106}$ and a variety of aqueous ${ }^{107}$, aqueous containing organic compounds $^{108}$, and non-aqueous ${ }^{109}$ formulation designs. It is most crucial for a wet etch clean $\operatorname{step}(\mathrm{s})$ to be robust with respect to polymer, residue and, metallic and non metallic particles removal, as well as exhibit high degree of compatibility to exposed substrate materials during the wet etch cleaning process.

The use of copper-compatible clean chemistries to remove these residues and provide ultra clean surface for the next processing step is a key for better device performance and reliability. If the cleaning solution attacks the copper line, the subsequent barrier and seed deposition processes may exhibit poor coverage over the 
excessively etched area, resulting in an incomplete via fill as shown in figure $3.2^{110}$. Therefore, understanding molecular mechanisms behind cleaning solutions is essential for developing successful Back-End-of-Line (BEOL) process.

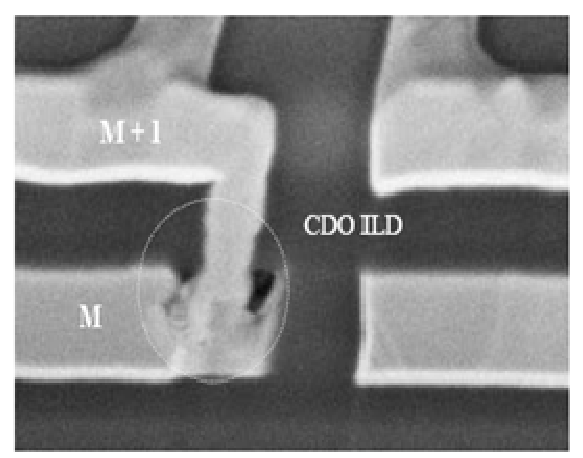

(a)

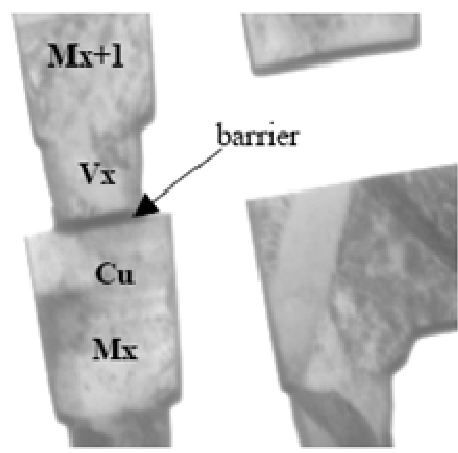

(b)

Figure 3.2 (a) Effect of copper attack by the wet etch clean chemistry during DD patterning. (b) TEM reference of a functional DD structure.

\subsection{Copper Rich Residue}

The presence of copper rich residue on the wafer surface after wet clean steps using non-copper compatible chemistry was detected. Examples of such residues are shown in Figure 3.3 and have been the driving force behind this study. The Energy Dispersive X-ray (EDX) histogram analysis of the residues/particles indicating the presence of $\mathrm{Cu}$ is shown in Figure 3.4. Copper corrosion/dissolution during cleaning process leads to the re-deposition/precipitation of copper and/or copper rich precipitants onto the wafer surface. The presence of copper on the ILD surface presents a serious yield and reliability problems to the performance of ULSI devices ${ }^{111}$, $112,113,114$. In order to obtain reliable devices with low via resistance, the residue on the sidewalls and bottom of the vias must be removed prior to the next process step. 
Similarly, copper rich residue may connect metal lines leading to undesired short circuits.

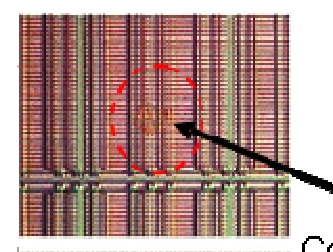

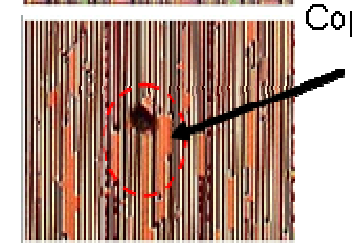

(a)

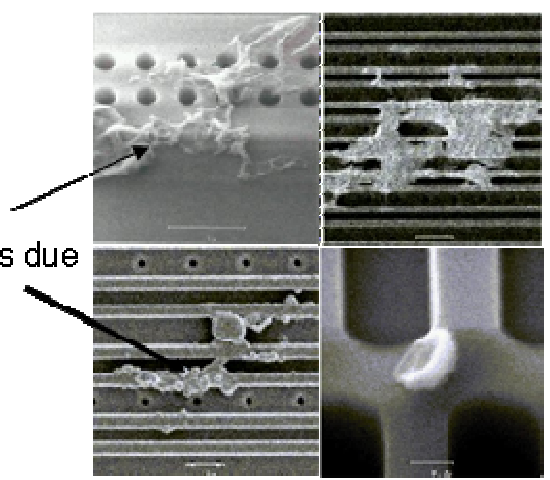

(b)

Figure 3.3 $\mathrm{Cu}$ containing residue observed after the wet etch clean step. (a) optical microscope image. (b) SEM micrograph ${ }^{115}$.

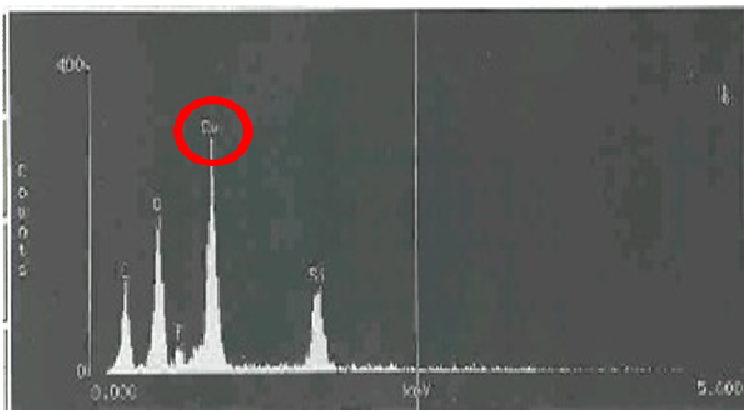

Figure 3.4 EDX histogram showing the composition of the residue on the ILD surface.

The objective of the study reported in this chapter is to investigate copper thin films corrosion/dissolution in aqueous solution containing HF, organic compounds, and $\mathrm{H}_{2} \mathrm{O}_{2}$ and its relevance to semiconductor DD patterning of copper interconnects. Such solutions are commonly used in the semiconductor industry for post plasma etch cleaning. Furthermore, we focus on the influence of hydrogen peroxide on copper corrosion from a mechanistic and kinetic stand point. Hydrogen peroxide is a common component in many cleaning formulations used for particle and residue 
removal in the fabrication of IC devices ${ }^{116,117,118,119,120,121,122}$. The chapter begins with a brief overview of principles and basic concepts of corrosion, $\mathrm{Cu}$ in particular. The discussion in subsequent sections focuses on methods used to quantify corrosion, corrosion prevention techniques, and copper dissolution kinetics and reaction mechanisms. Several plausible models to explain the observed undesired copper deposition onto the ILD surface are also discussed.

\subsection{Properties of Copper}

Copper has played a significant part in the history of humankind, which has used the easily accessible uncompounded metal for thousands of years. Copper is the only metal found in its metallic state ready for use, and it's relatively easy to shape. Copper softness is due to its face centered cubic atomic structure. Copper can be alloyed with other metals to achieve hardness. For example, bronze is an alloy of copper and tin, and brass is an alloy of copper and zinc. Copper usage is second only to $\operatorname{iron}^{123,124}$.

Copper has been one of the most important metals used in advancement of technology. This is primarily due to its high electrical and thermal conductivity, second only to silver among pure metals at room temperature ${ }^{125}$. Copper is a reddishcolored metal; it has its characteristic color because of its band structure. In its liquefied state, a pure copper surface without ambient light appears somewhat greenish, a characteristic shared with gold. When liquid copper is in bright ambient light, it retains some of its pinkish luster. 
Copper is the first element of group IB of the periodic table, has atomic

number 29 and occurs in two isotopes, ${ }^{63} \mathrm{Cu}$ and ${ }^{65} \mathrm{Cu}$. Copper occupies the same family of the periodic table as silver and gold, since they each have one s-orbital electron on top of a filled electron shell. This similarity in electron structure makes them similar in many characteristics. All have very high thermal and electrical conductivity, and all are malleable metals. The presence of any impurities lowers copper's conductivity. Copper oxides are unstable relative to other metals due to low free energy of formation.

Copper has immense commercial importance. Millions of tons are used annually. Copper is used in many applications such as wires, coinage, tools, electrical conductor testing and casting alloys. A principle use is in the electrical and electronic industries for interconnects applications ${ }^{96}$.

\subsection{Principles of corrosion:}

\subsubsection{Definition:}

Corrosion of metallic materials is the destructive result of chemical reaction between a metal or metal alloy and its environment.

\subsubsection{Basic Concepts:}

At present, insufficient knowledge is available to predict with any certainty how a particular metal or alloy will behave in a specific environment. The mechanical and physical properties can be expressed in terms of constants; the chemical properties of a given metal are dependent entirely on the precise environmental conditions during processing. The relative importance of mechanical, physical and chemical properties 
will depend in any given case on the application of the metal. For example, for ULSI interconnect systems, electrical conductivity, adhesion, electromigration resistance, and corrosion resistance are of major importance.

One can use thermodynamics, e.g., Pourbaix or E-pH diagrams, to evaluate the theoretical activity of a given metal or alloy provided the chemical makeup of the environment is known.

\subsubsection{Pourbaix Diagram}

E-pH or Pourbaix diagrams are a convenient way of summarizing much thermodynamic data and provide a useful means of summarizing the thermodynamic behavior of a metal and associated species in given environmental conditions. E-pH diagrams are typically plotted for various equilibria on normal Cartesian coordinates with potential $(E)$ as the ordinate $(y$ axis $)$ and $\mathrm{pH}$ as the abscissa $(x \text { axis })^{126}$. For corrosion in aqueous media, two fundamental variables, namely corrosion potential and $\mathrm{pH}$, are deemed to be particularly important. Changes in other variables, such as the oxygen concentration, tend to be reflected by changes in the corrosion potential. The $E$-pH diagrams is simply designed for a metal/water system that shows the condition of oxidizing power versus the acidity or alkalinity $(\mathrm{pH})^{127,128,129}$. The Pourbaix diagram describes the reaction of a metal in a water system to indicate regions of immunity, corrosion/dissolution, and passivation. The diagram is constructed based on the Nernst potential of a metal versus hydrogen electrode and is plotted against $\mathrm{pH}$ of an electrolyte. All possible reactions are shown in the diagram to indicate where each phase/species of a metal is present. Figure 3.5 shows a diagram 
for the copper and water system. There are three main regions within the Pourbaix diagram: corrosion, where metal in the form of cations and anions is present, passivity or passivation region where a stable metal oxide generally forms on the metal and finally, the immunity region in which the metal itself (reduced form) is present and no reaction occurs. Within the Pourbaix diagram, two lines are also drawn where stability of hydrogen and oxygen gases and water is indicted to assist in potential reactions that may be expected. The regions that allow for thermodynamically stable compound are referred to as the passive region.

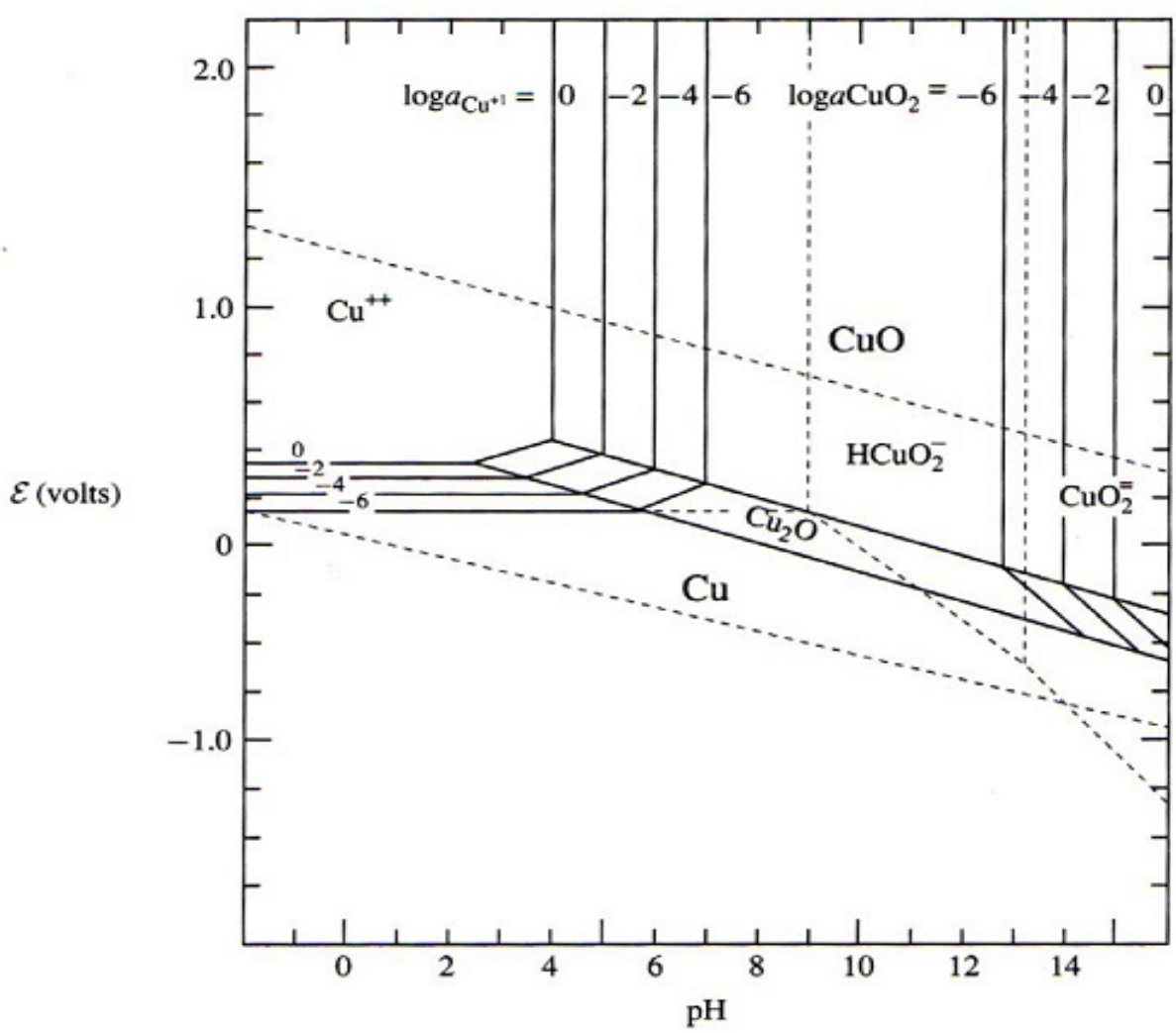

Figure 3.5 Potential-pH diagram of copper in water system ${ }^{130}$. 


\subsubsection{Pourbaix Diagram Limitations}

For the usual conditions in areas on the Pourbaix diagram where corrosion is possible, no information on corrosion kinetics is provided by these thermodynamically derived diagrams. The diagrams are derived for specific temperature and pressure conditions and selected concentrations of ionic species. Most diagrams consider pure substances only; the presence of other species can form complexes with the metal. Therefore, additional computations must be made if other species are involved. In areas where a Pourbaix diagram shows oxides to be thermodynamically stable, these

oxides are not necessarily of a protective (passivating) nature ${ }^{131,132}$. Lastly, the Pourbaix diagrams as the one shown in Figure 3.5 have been established based on the behaviors of bulk copper and not copper thin films which are the focus of this investigation.

\subsubsection{Corrosion Properties of Copper:}

Copper naturally occurs in the environment and it is obtained by reduction of its compounds. It is not very reactive chemically based on the electrochemical series $^{133}$, and oxidizes slowly in atmosphere at room temperature. It has a relatively noble potential based on the EMF series and galvanic series ${ }^{134}$. Very small amounts of copper added to a solution may cause considerable corrosion of more anodic metals elsewhere in the system ${ }^{105}$. Copper alloys are more corrosion resistant than copper itself due to more protective and stable film formation.

A thermodynamic warning of the likelihood of corrosion is obtained by comparing the standard potentials of the copper reduction, such as 


$$
\begin{array}{ll}
\mathrm{Cu}^{2+}(a q)+2 e^{-} \rightarrow \mathrm{Cu}(s) & E^{\varnothing}=+0.342 \mathrm{~V} \\
2 \mathrm{H}^{+}(a q)+2 e^{-} \rightarrow \mathrm{H}_{2}(g) & E^{\varnothing}=0 \mathrm{~V} \\
4 \mathrm{H}^{+}(a q)+\mathrm{O}_{2}(g)+4 e^{-} \rightarrow 2 \mathrm{H}_{2} \mathrm{O}(l) & E^{\varnothing}=+1.23 \mathrm{~V}
\end{array}
$$

Because equation (c) redox standard potential is more positive than $E^{\varnothing}\left(C u^{2+} / C u\right)$, it can drive its oxidation.

$$
\begin{aligned}
& E(\mathrm{a})=E^{\varnothing}(\mathrm{a})+\frac{R T}{F} \ln a\left(H^{+}\right)=-0.059 \mathrm{~V} \times p H \\
& E(\mathrm{~b})=E^{\varnothing}(\mathrm{b})+\frac{R T}{F} \ln a\left(H^{+}\right)=1.23 \mathrm{~V}-0.059 \mathrm{~V} \times p H
\end{aligned}
$$

The above expression provides an indication at what $\mathrm{pH}$ the copper will have a tendency to oxidize.

\subsection{Experimental}

\subsubsection{Substrate}

The experiments were performed using copper on blanket silicon wafers $(\mathrm{Cu} / \mathrm{Si})$ substrate. The copper film was uniformly deposited using typical industry electroplating (EP) equipment ${ }^{135,136}$ followed by a chemical mechanical planarization (CMP) step to closely mimic the actual $\mathrm{Cu}$ surface during the DD process. The thickness of the $\mathrm{Cu}$ film is approximately $400 \mathrm{~nm}$ as determined by 4 point probe method (Surface resistivity meter, Gaurdian Manufacturing Inc., Model SRM-232) and cross-section SEM.

\subsubsection{Cleaning solution}

The electrochemical and dissolution experiments were performed utilizing a semiconductor cleaning chemical solution supplied by Ashland Chemicals, which was 
composed of $93 \mathrm{wt} \%$ ethylene glycol, $4 \mathrm{wt} \%$ ammonium fluoride, $0.033 \mathrm{wt} \%$ hydrofluoric acid (HF), and $\sim 3 \mathrm{wt} \%$ water, known as Dilute EG3. All experiments were carried out at room temperature $\left(21^{\circ} \mathrm{C}\right)$.

\subsubsection{Surface Preparation}

Before proceeding with electrochemical or immersion experiments, a surface treatment with $0.49 \mathrm{wt} \% \mathrm{HF}$ was carried out to ensure a clean surface. A set of screening experiments to investigate optimal dip time in HF solution for surface cleaning were conducted. A one minute HF treatment was found to be appropriate. This pretreatment renders a clean surface on which the native copper oxide is partially removed to expose a clean elemental copper surface. Longer exposure $(\approx 5$ minutes $)$ of the wafer to HF did not alter the X-ray Photoelectron Spectroscopy (XPS) spectra, which exhibited peak due to $\mathrm{Cu}(\mathrm{I})$ and $\mathrm{Cu}(0)$. See Figure 3.16 (red line).

\subsubsection{Experimental Conditions}

In the electrochemical experiments, copper corrosion was investigated under deareated, partially-deareated and non-deareated solution conditions. In the immersion experiments several factors were investigated. These included: Nondeareated, deareated, aerated, addition of hydrogen peroxide $\left(\mathrm{H}_{2} \mathrm{O}_{2}\right)$, and in the presence of organic corrosion inhibitors. Deaeration was achieved by bubbling nitrogen through the solution, and aeration was accomplished by bubbling air into the solution. Dissolved oxygen (DO) concentration measurements were performed using a digital oxygen meter (Traceable ${ }^{\circledR}$ Dissolved Oxygen Meter, Control Company, 
Model RS-232). In the dissolution experiments, copper concentration was monitored using ICP-MS.

\subsubsection{Electrochemical measurements}

Open-circuit potential and potentiodynamic experiments were carried out on the $\mathrm{Cu} / \mathrm{Si}$ wafers. The electrochemical experiments were conducted in a threeelectrode electrochemical flat cell. Platinum mesh was used as the counter electrode. All potentials were measured versus a saturated calomel electrode (SCE) which represents the reference electrode. $\mathrm{Cu}$ wafer with an area of $0.3 \mathrm{~cm}^{2}$ defined by a viton O-ring was used as the working electrode. A Princeton Applied Research Model 273 potentiostat/galvanostat interfaced with an IBM compatible computer was used for data collection. Corrware/corrview software (Scribner Associates) was used to conduct the experiments and the data analysis. Experiments were performed in triplicates to ensure reproducibility. For the deareated and partially-deareated experiments, nitrogen was bubbled into the solution in a round bottom flask and then transferred into the electrochemical cell. The concentration of DO in the deareated, partially-deareated and non-deareated solutions is $0.6,2.1$, and $4.5 \mathrm{mg} / 1$, respectively.

\subsubsection{Open-circuit potential experiments}

In these experiments, open-circuit potential (OCP) values or free corrosion potentials were determined for $\mathrm{Cu}$ by immersing the $\mathrm{Cu} / \mathrm{Si}$ sample in to the cleaning solution for at least ten minutes. 


\subsubsection{Potentiodynamic experiments}

Polarization curves were obtained in order to determine the influence of the cleaning solution on the corrosion behavior of the investigated wafers. In each experiment, the potential was scanned at a rate of $10 \mathrm{mV} / \mathrm{min}$ in the anodic direction, starting from a potential level of $100 \mathrm{mV}$ more negative than the open-circuit potential.

\subsubsection{Dissolution experiments}

Dissolution experiments were carried out using $16 \mathrm{~cm}^{2}(4 \mathrm{~cm} \mathrm{x} 4 \mathrm{~cm})$ copper/silicon wafer coupons in a $300 \mathrm{ml}$ volume of cleaning solution. Aliquots from the cleaning solution containing the copper wafer were collected for inductively coupled plasma mass spectrometric (ICP-MS) analysis at specific intervals to determine copper concentrations. Experiments were performed in 3 replicates to ensure reproducibility. ICP-MS measurements were performed using the Agilent $7500 \mathrm{CS}$

The influence of a strong oxidant was investigated by the addition of $\mathrm{H}_{2} \mathrm{O}_{2}$ (J.T. Baker, Inc.) to the cleaning solution. The final $\mathrm{H}_{2} \mathrm{O}_{2}$ concentration in the solution was 5\%. The effect of a commercial corrosion inhibitor, benzotriazole (BTA), was also investigated. For such studies the cleaning solution contained BTA typically at $0.5 \%$ concentration level to suppress the copper dissolution in cleaning solution.

\subsubsection{Surface characterization}

SEM images and Energy Dispersive X-ray (EDX) analysis were performed using the Hitachi S-4700. Atomic Force Microscopy (AFM) images were produced using Atomic Force Profiler (Dimension, Model $\mathrm{V}_{\mathrm{x}} 330$ ). The X-ray Photoelectron 
Spectroscopy (XPS) survey spectra were obtained using a VG Scientific Theta Probe 300.

\subsection{Results}

\subsubsection{Electrochemical Corrosion Measurements}

\subsubsection{Open-Circuit Potential Measurements}

The open-circuit potential (OCP) measurements for $\mathrm{H}_{2} \mathrm{O}_{2}$ containing, nondeareated and partially deareated cleaning solution with $\mathrm{Cu}$ film as a working electrode are shown in Figures 3.6, a, b and c, respectively. In all cases, the OCP decays exponentially to final equilibrium value suggesting that the copper thin film is becoming more active in these solutions. In the case of cleaning solution containing strong oxidizer, $\mathrm{H}_{2} \mathrm{O}_{2}$, the $\mathrm{OCP}$ value settles at $0.291 \mathrm{~V}$ starting from $0.301 \mathrm{~V}$, i.e. about $10 \mathrm{mV}$ change over 5 minutes.

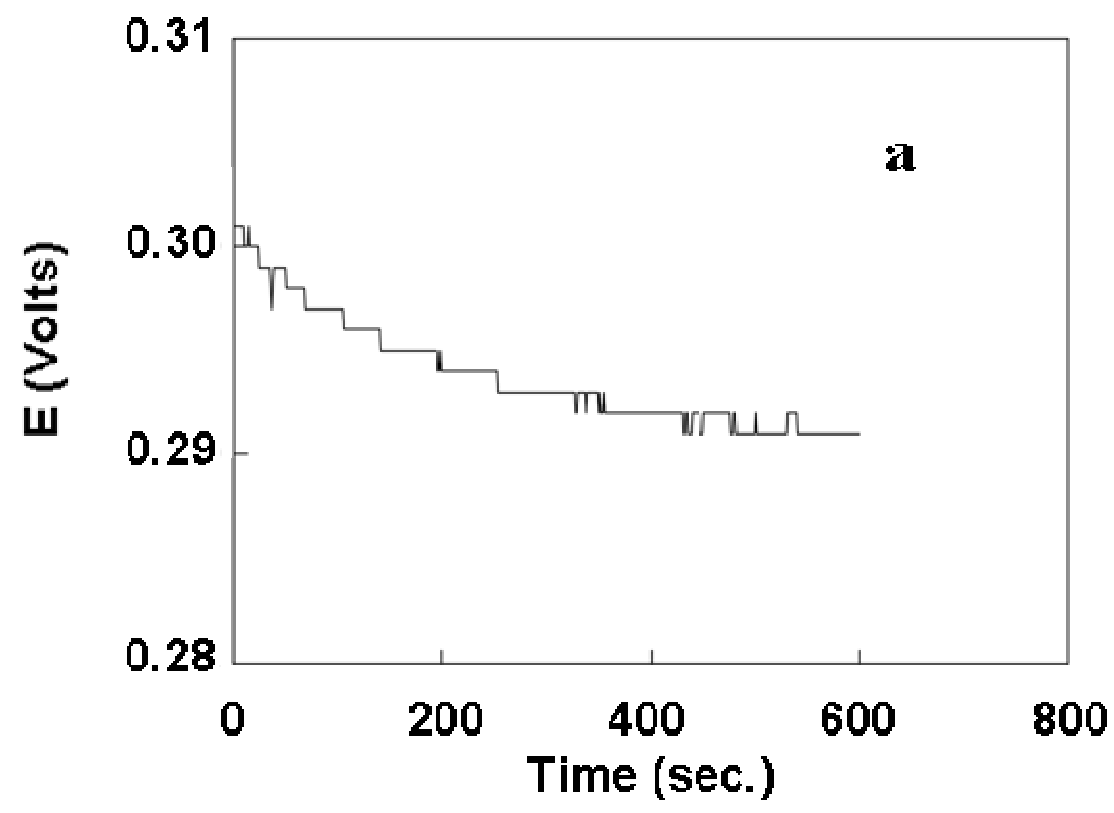



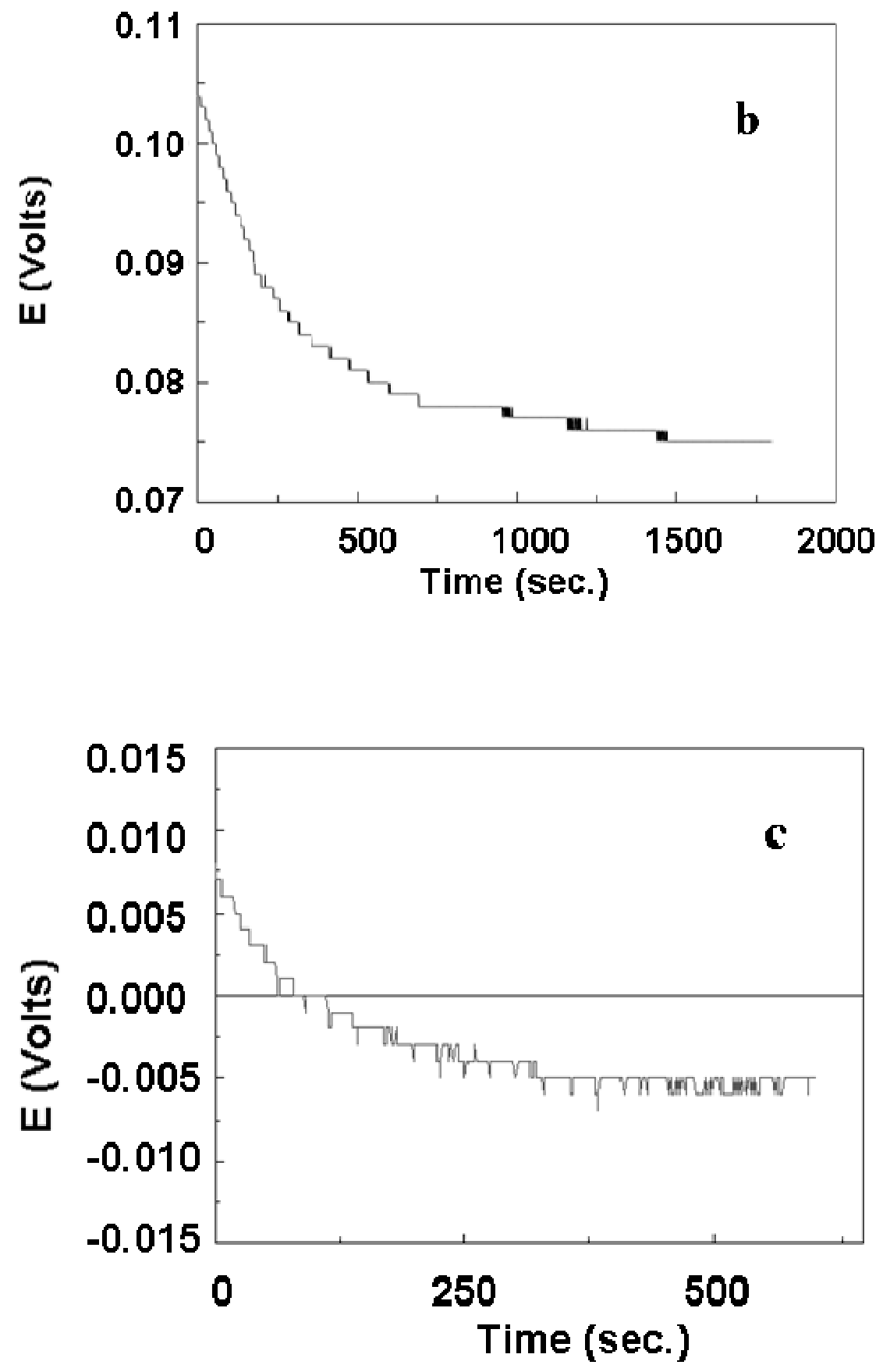

Figure 3.6 Figure 3. Open circuit potential measurements for electrolytic solutions used; (a) in presence of $5 \% \mathrm{H}_{2} \mathrm{O}_{2}$, (b) in an non-deareated (DO $4.5 \mathrm{mg} / \mathrm{L}$ ) and (c)deareated (DO $0.6 \mathrm{mg} / \mathrm{L}$ ) solutions. The corresponding OCPS are $0.29,0.08$ and 0.005 Volts respectively. Note the equilbriation period is longer in non deareated solutions. 


\subsubsection{Potentiodynamic Experiments}

The results of the potentiodynamic experiments, namely: anodic polarization curves for copper obtained in the hydrogen peroxide containing, and partially-deareated and non-deareated cleaning solutions are shown in Figure 3.7 a and b, respectively.
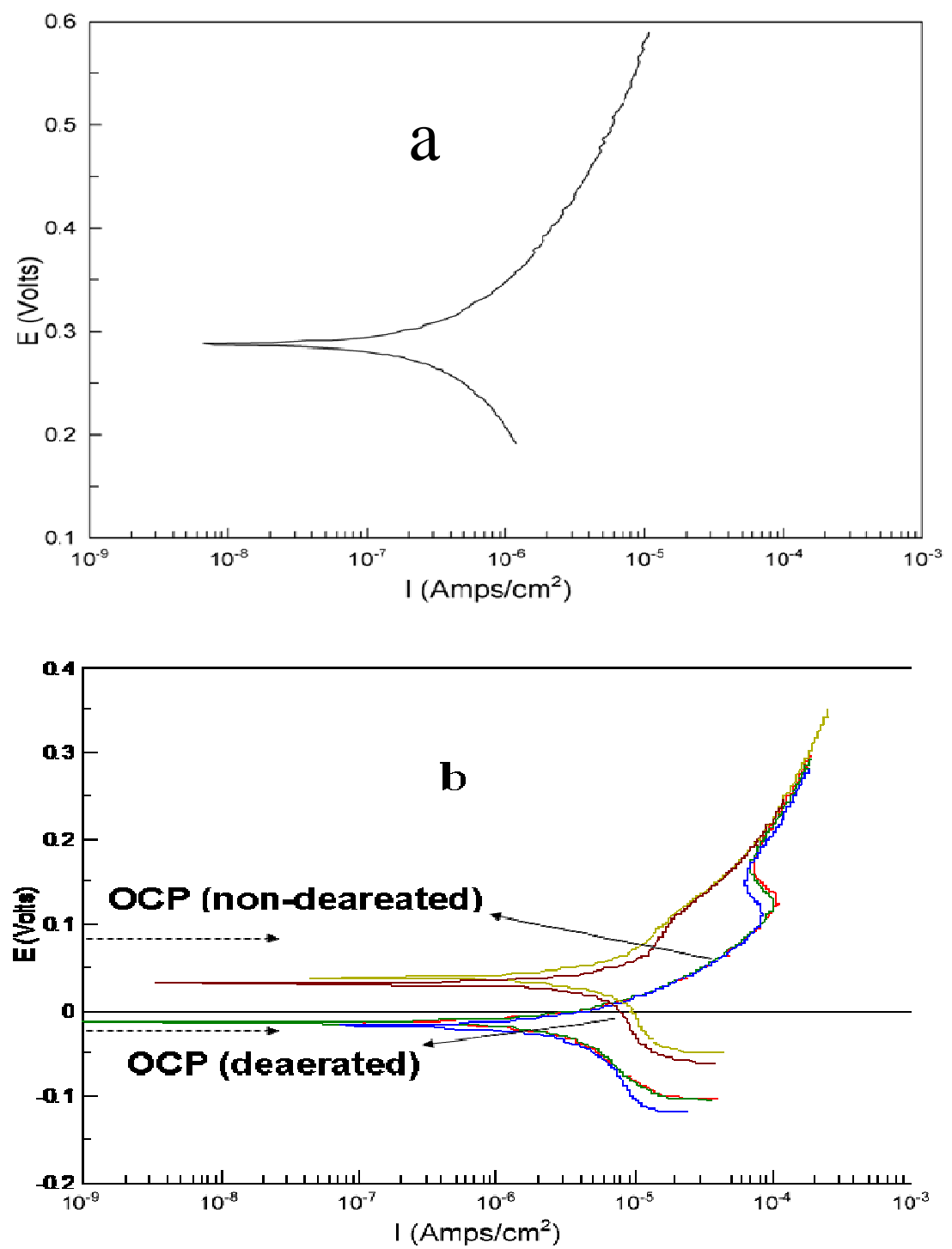

Figure 3.7 Potentiodynamic curves for copper thin films on $\mathrm{Si}$ in cleaning solution containing (a) $\mathrm{H}_{2} \mathrm{O}_{2}$ and under (b) non-deareated and deareated conditions. The dashed arrows indicate positions of OCPs while the full arrows indicate the corresponding potentiodynamic polarization curves. 


\subsubsection{Immersion measurements}

From a thermodynamic point of view, the electrochemical experiments indicated active dissolution of copper in the dilute EG3 solution. Therefore, to complement the electrochemical work, $\mathrm{Cu}$ dissolution experiments employing ICPMS techniques were carried out to determine the dissolution rate and reaction kinetics of copper in dilute EG3.

\subsubsection{Dissolution experiments in non-deareated dilute EG3}

The experimental results for $\mathrm{Cu}$ dissolution in non-deareated dilute EG3 are shown in Figure 3.8.

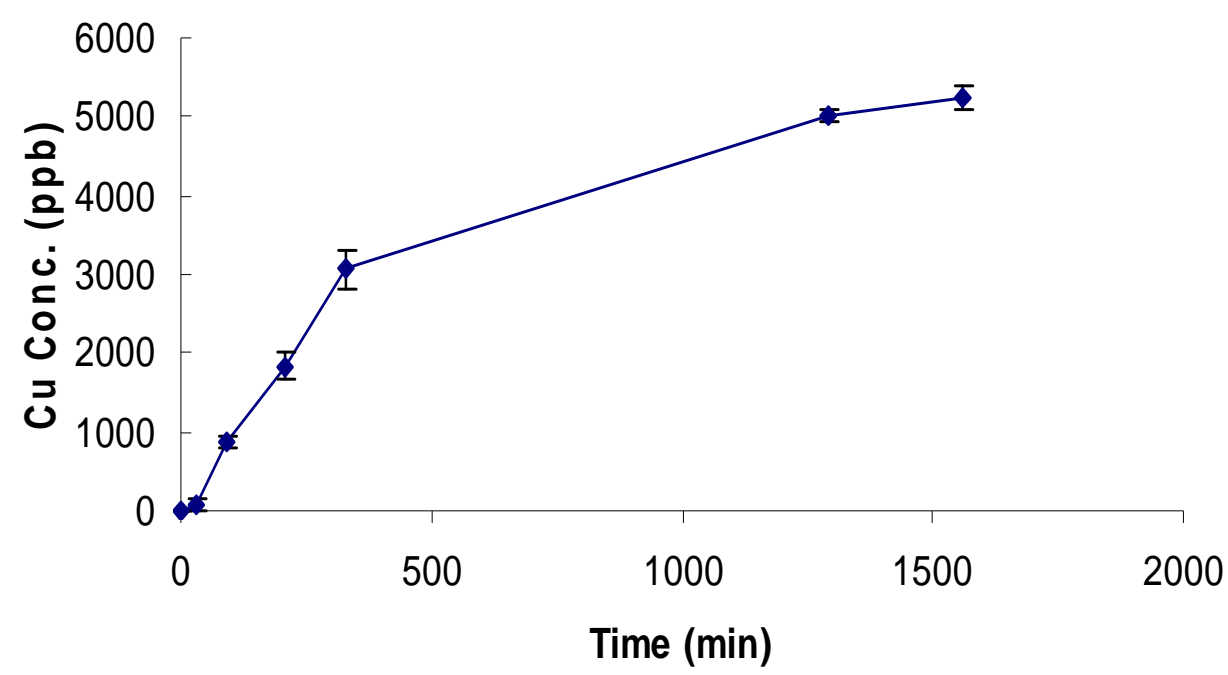

Figure 3.8 $\mathrm{Cu}$ dissolution in non-deareated dilute EG3 as a function of time. Dissolved oxygen concentration was $4.5 \mathrm{mg} / \mathrm{L}$.

\subsubsection{Copper Dissolution in aerated dilute EG3}

The experimental results for copper dissolution in aerated dilute EG3 are shown in Figure 3.9. 


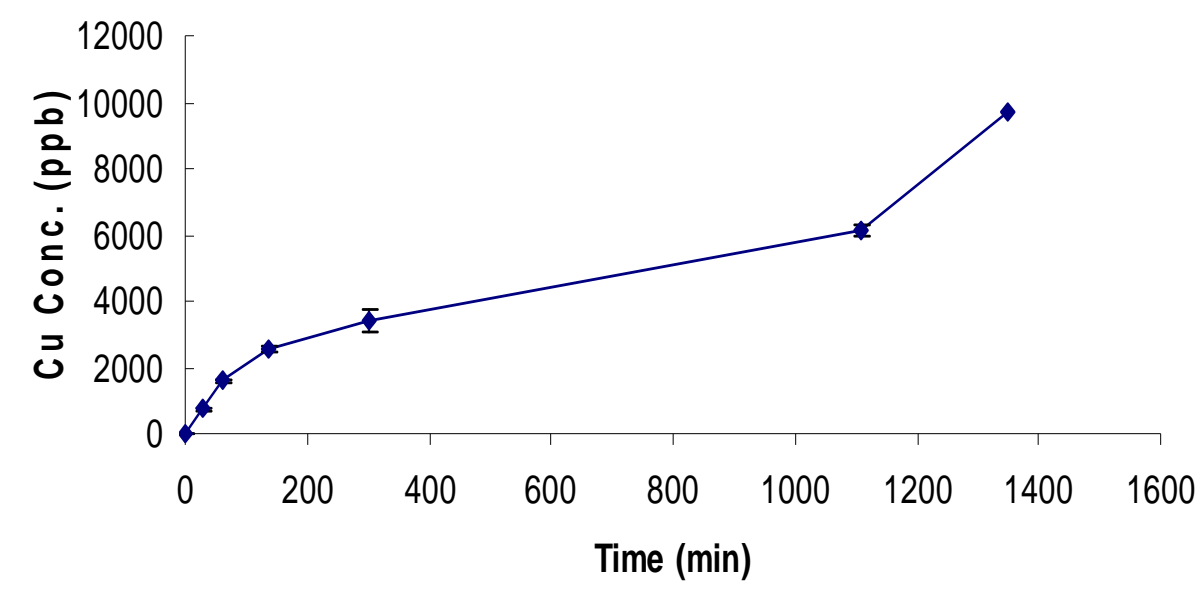

Figure 3.9 $\mathrm{Cu}$ dissolution in aerated dilute EG3 as a function of time. Dissolved oxygen concentration was $8.9 \mathrm{mg} / \mathrm{L}$.

\subsubsection{Copper Dissolution in deareated dilute EG3}

The experimental results for $\mathrm{Cu}$ dissolution in deareated dilute EG3 are shown in Figure 3.10.

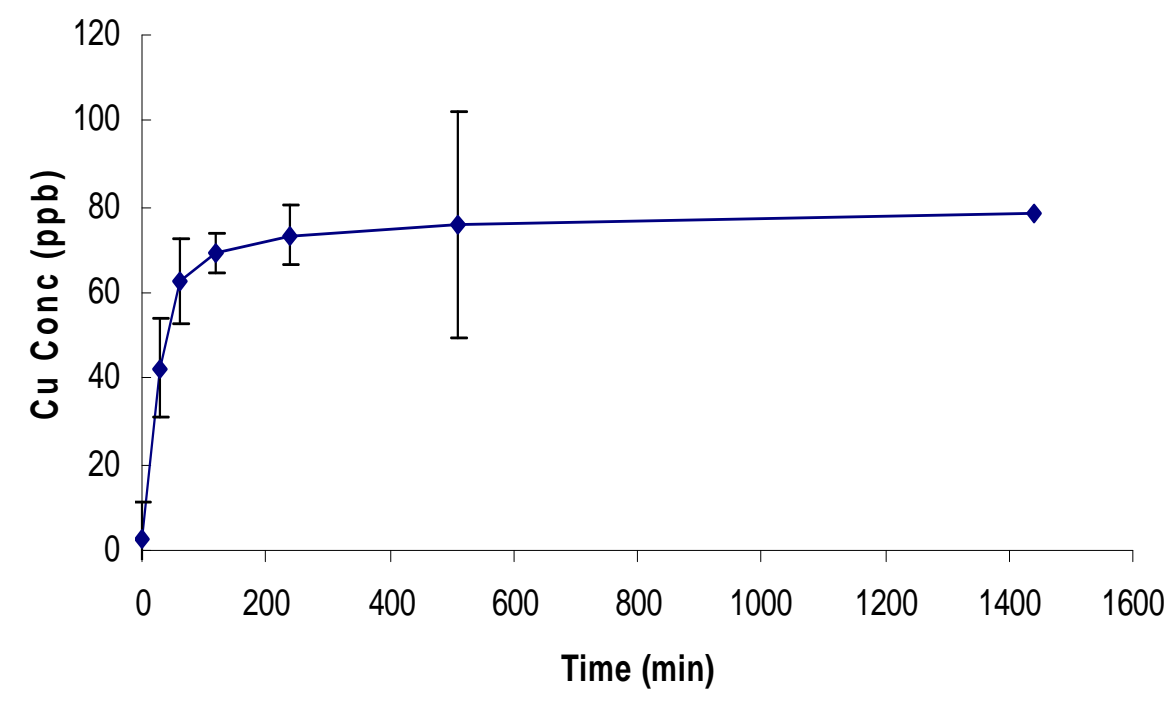

Figure 3.10 $\mathrm{Cu}$ dissolution in deareated dilute EG3 as a function of time. Dissolved oxygen concentration was $0.6 \mathrm{mg} / \mathrm{L}$. 


\subsubsection{Copper Dissolution in non-deareated dilute EG3 in the presence of $\mathrm{H}_{2} \mathrm{O}_{2}$}

Copper dissolution results for the non-deareated dilute EG3 and in the presence of $\mathrm{H}_{2} \mathrm{O}_{2}$ is shown in Figure 3.11.

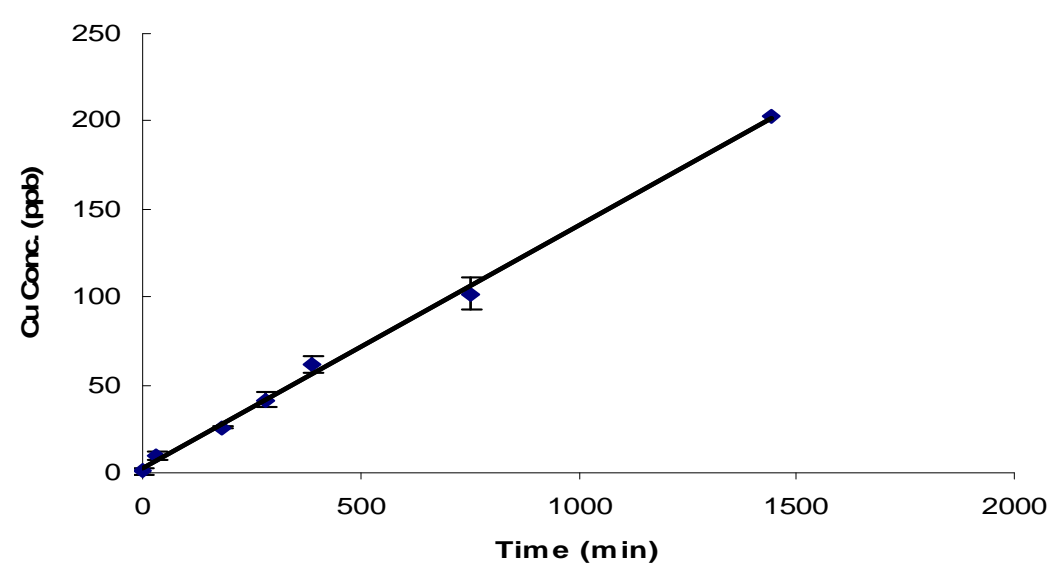

Figure 3.11 $\mathrm{Cu}$ dissolution in non-deareated dilute EG3 in the presence of $\mathrm{H}_{2} \mathrm{O}_{2}$ as a function of time.

\subsubsection{Copper Dissolution in non-deareated dilute EG3 in the presence of BTA}

Copper dissolution results for non-deareated dilute EG3 in the presence of an organic corrosion inhibitor benzotriazole (BTA) are shown in Figure 3.12.

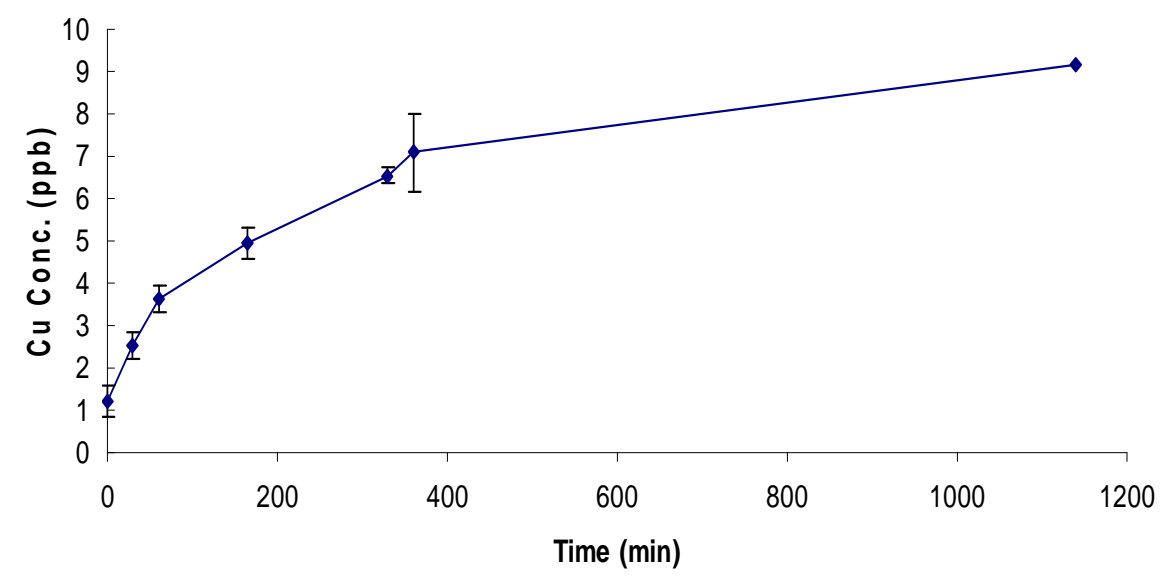

Figure 3.12 $\mathrm{Cu}$ dissolution in non-deareated dilute EG3 in the presence of BTA as a function of time. 


\subsubsection{Surface characterization}

Since copper undergoes active dissolution in dilute EG3, it is important to study the surface characteristics of the copper thin film after the cleaning process.

\subsubsection{Atomic Force Microscopy (AFM)}

Atomic force microscopy investigation was carried out to complement the SEM work to determine if the dilute EG3 solution has any impact on the copper surface and confirm the type of corrosion, namely: uniform corrosion. Localized corrosion, such as pitting corrosion is not observed in the atomic force microscopic images given in Figure 3.13 and 3.14 before and after immersion respectively, where the mean surface roughness before and after exposure to cleaning solution is $3.8 \pm$ $0.1 \mathrm{~nm}$, and $0.82 \pm 0.04 \mathrm{~nm}$ respectively.

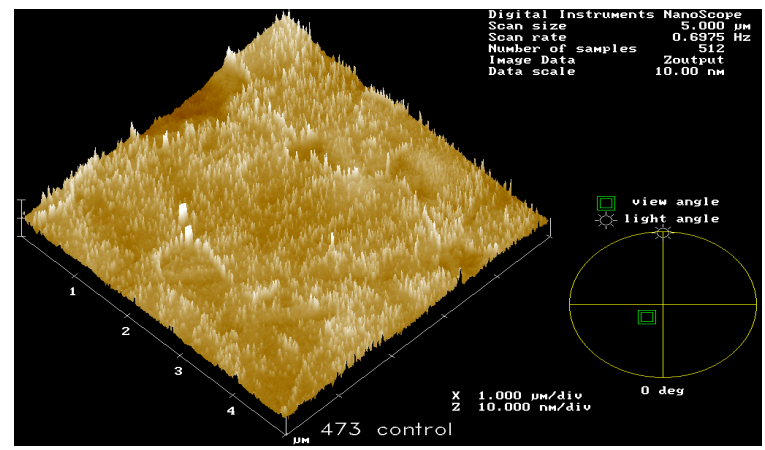

Figure 3.13 AFM image of the copper wafer before immersion in dilute EG3.

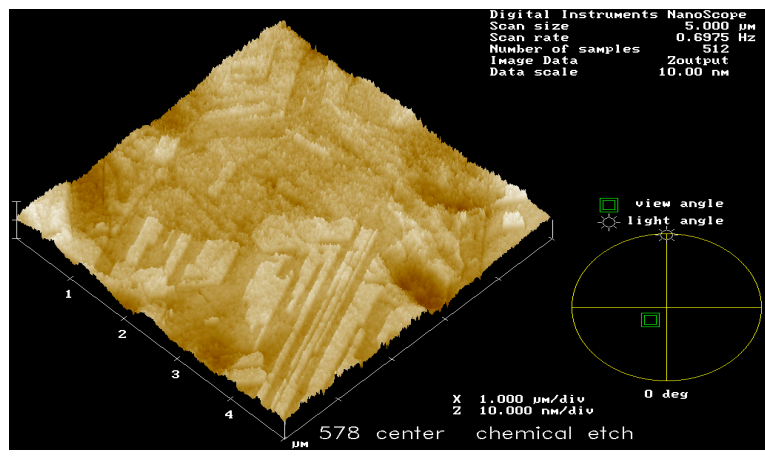

Figure 3.14 AFM image of the copper wafer after immersion in dilute EG3. 


\subsubsection{X-ray Photoelectron Spectroscopy (XPS)}

The XPS spectra, Figure 3.15, show the surface compositional changes that take place before and after the cleaning treatment. The vertical lines indicate positions for the various oxidation states of copper. Virgin copper wafers show $\mathrm{Cu}_{2} \mathrm{O}$ while cleaned wafers indicate the presence of both $\mathrm{Cu}_{2} \mathrm{O}$ and $\mathrm{Cu}^{0}$. The interface between copper and the cleaning solution differs in the presence of $\mathrm{H}_{2} \mathrm{O}_{2}$. The XPS results shown in Figure 3.16 reveal a replacement of $\mathrm{Cu}_{2} \mathrm{O}$ with $\mathrm{CuO}$ on the copper surface, perhaps due to oxidation by $\mathrm{H}_{2} \mathrm{O}_{2}$.

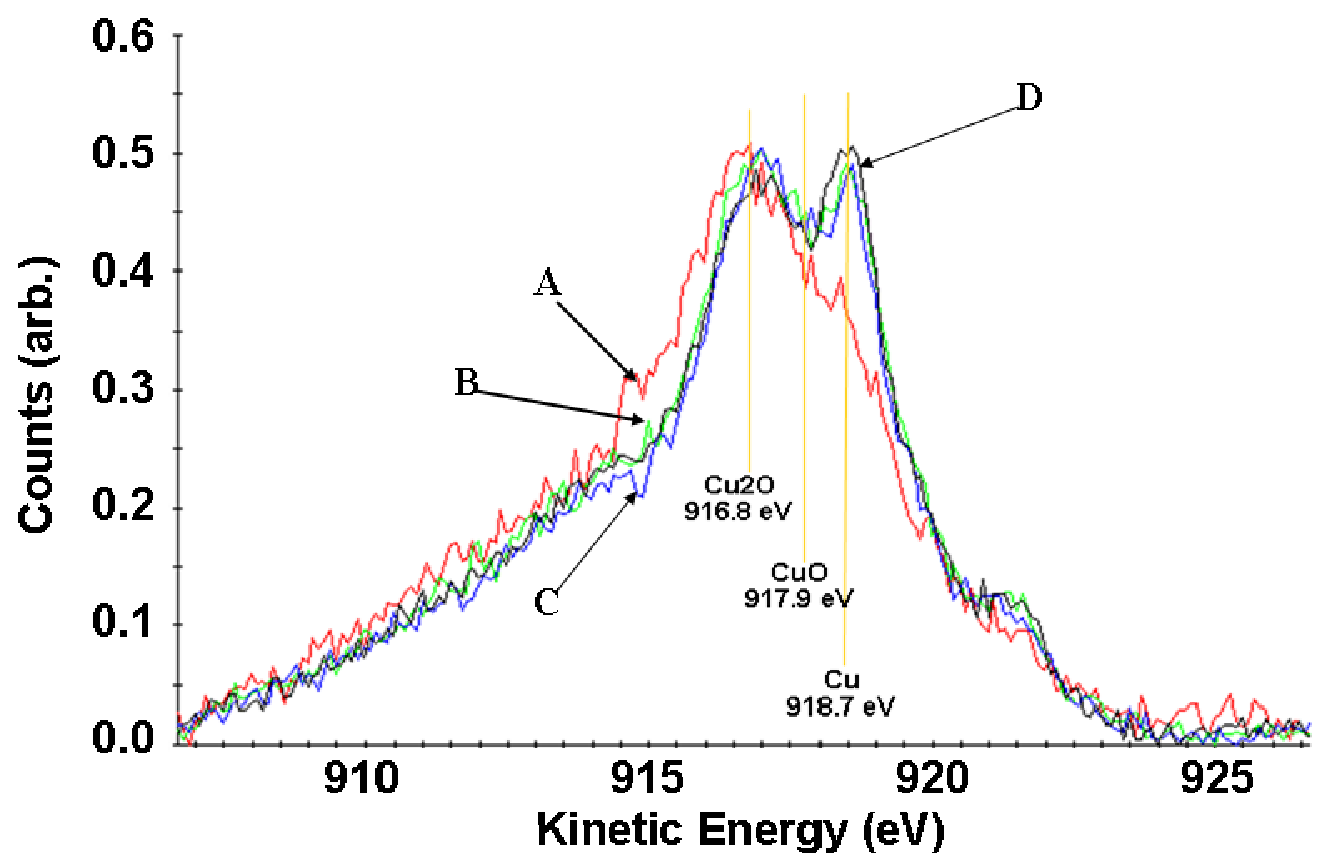

Figure 3.15 XPS spectra of the copper surface before and after surface treatments. A line corresponds to wafer as received (control), B line is the XPS spectrum after surface treatment with $0.49 \% \mathrm{HF}$, the $\mathbf{C}$ and $\mathbf{D}$ lines correspond to wafers that were immersed in the cleaning solution for 6 and 12 hours, respectively. 


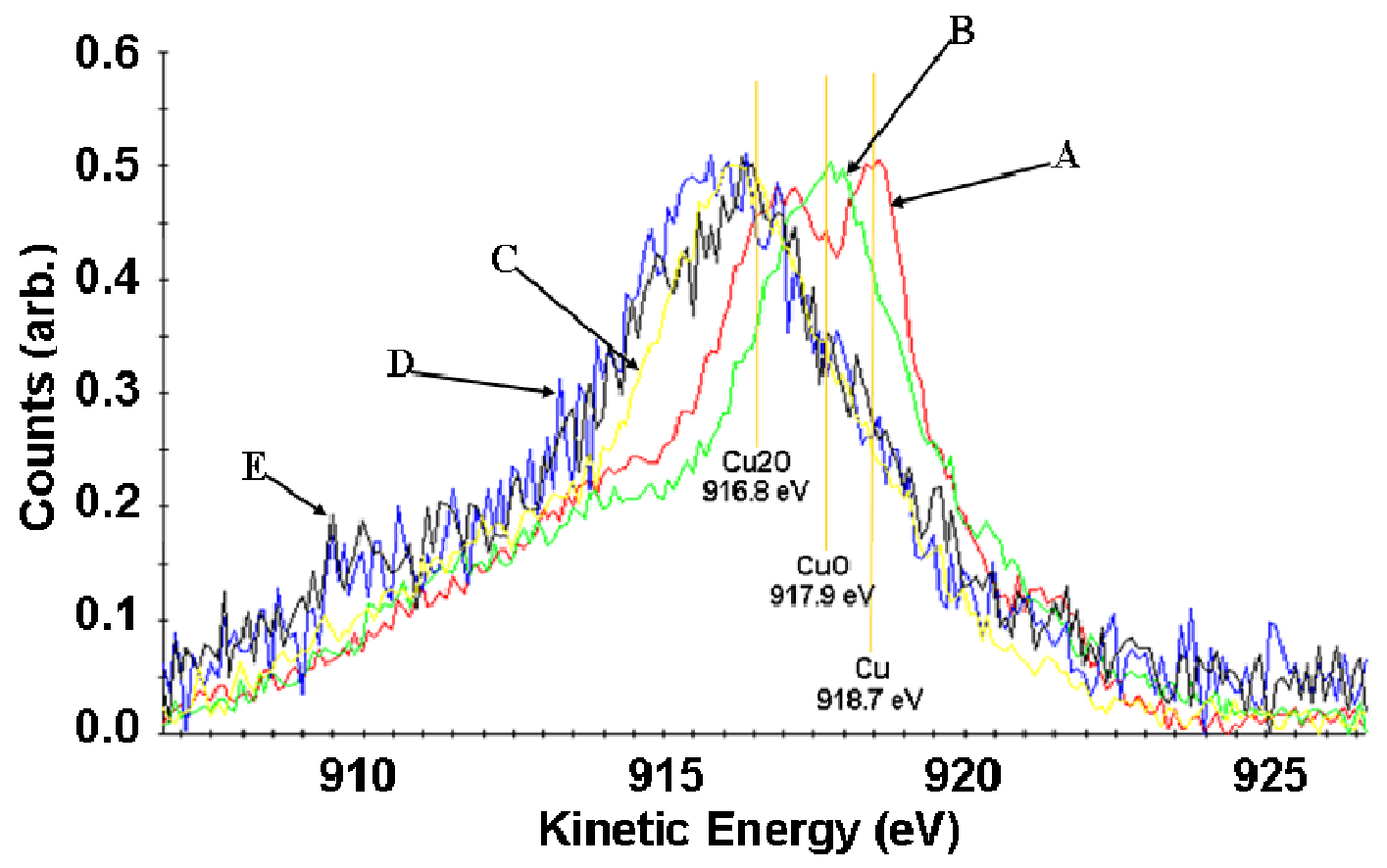

Figure 3.16 XPS spectra of the copper surface before and after wet cleaning solution treatments. The $\mathbf{A}$ line corresponds to wafers that were dipped in dilute cleaning solution. B line corresponds to wafer processed in dilute cleaning solution containing $5 \% \mathrm{H}_{2} \mathrm{O}_{2}$. The other lines correspond to wafers processed in cleaning solution with commercial organic corrosion inhibitor benzotriozol at different concentrations, C $(0.1 \%), \mathbf{D}(0.5 \%)$, and $\mathbf{E}(1.0 \%)$.

\subsection{Discussion}

The OCP decays exponentially to final equilibrium value suggesting that the copper thin film is becoming more active in these solutions. In the case of cleaning solution containing strong oxidizer, $\mathrm{H}_{2} \mathrm{O}_{2}$, the OCP value settles at $0.291 \mathrm{~V}$ starting from $0.301 \mathrm{~V}$, i.e. about $10 \mathrm{mV}$ change over 5 minutes. The open-circuit stability indicates that the corrosion of copper is under activation polarization. Intersection of the OCP on the potentiodynamic curve shown in Figure 3.7a indicates that the copper is under active corrosion. The shift of corrosion potential to a nobler potential in 
Figure 3.7a, in comparison to deareated and non-deareated samples (Figure 3.7b), is consistent with the effect of a strong oxidizer ${ }^{137}$ on active-passive corroding system.

The time scale for establishing OCP is longer in a non-deareated solution implying a diffusion controlled process. The OCP potential change in a non-deareated solution is $\sim 30 \mathrm{mV}$ taking place over 25 min duration. In addition, comparing the OCP value to the active potential values in the polarization curve of Figure 3.7 indicates that copper is active at the open circuit potential. The OCP values depend upon concentration of dissolved oxygen. Any reduction in oxygen concentration results in reduction of limiting current density and potential values for the cathodic reaction causing the open-circuit decay. This indicates that corrosion of copper is under concentration polarization. In the deareated solution, the potential change is only $\sim 10 \mathrm{mV}$ during first few minutes and reaches a steady state after $\sim 5 \mathrm{~min}$. The open-circuit stability shows that the corrosion of copper is under activation polarization.

The potentiodynamic polarization curves show that the corrosion of copper in the cleaning solution increase to $0.2 \mathrm{~V}$ is affected by dissolved oxygen (DO) concentration. Higher current values are observed for $\mathrm{Cu}$ in non-deareated solution up to $0.2 \mathrm{~V}$. Above this potential, the current values are similar for $\mathrm{Cu}$ in the deareated and non-deareated cleaning solutions. The shape of the anodic polarization curves for $\mathrm{Cu}$ in the deareated and oxygen-containing solutions is different from each other. In the deareated solution, the current values increase with increasing the potential values above $\sim 0.05 \mathrm{~V}$. This indicates the presence of an active region in which metal 
dissolution takes place. In the non-deareated solutions, the current values increase with increasing potential values from $\sim-0.02 \mathrm{~V}$ to $0.12 \mathrm{~V}$ indicating the presence of an active region. Then, the current values decrease with increasing potentials to $\sim 0.14 \mathrm{~V}$ indicating the presence of an active-passive region. A constant current is observed between _ $\sim 0.14 \mathrm{~V}$ and $\sim 0.17 \mathrm{~V}$ indicating the presence of a passive region. Above the passive region, the current values increase with increasing the potential values applied. The critical passivation current $\left(10^{-4} \mathrm{~A} / \mathrm{cm}^{2}\right)$ is slightly higher in the non-deareated solution than that $\left(8.5 \times 10^{-5} \mathrm{~A} / \mathrm{cm}^{2}\right)$ in the partially-deareated solution. In the active region, $\mathrm{Cu}^{2+}$ is likely formed, which is supported by the Pourbaix diagram for $\mathrm{Cu}-$ water system ${ }^{138}$. Relatively high current is observed in the passive region. This indicates that non-protective film is formed on the surface. Given the sensitivity of the potentiodynamic scans to dissolved oxygen, the film could be due to formation of oxide(s) of copper, as explained in the next section.

The shift in corrosion potential for peroxide containing solutions is high extending well above $0.2 \mathrm{~V}$. Above $0.287 \mathrm{~V}$, the current increases with potential an indication of an active region. However unlike the non-deareated/partially deareated samples, no clearly defined active to passive transition is visible in the plot. The low corrosion current density observed at corrosion potential is consistent with lower copper dissolution rates measured by ICP-MS. These potentiodynamic polarization experiments were analyzed by employing Corrview software to determine the corrosion rate in terms of the corrosion current density $\left(\mathrm{I}_{\text {corr }}\right)$ using Tafel slopes. The 
corrosion rates for the deareated and non-deareated experiments are $0.80 \pm 0.04$ $\mathrm{A} \%$ min. and $2.0 \pm 0.1 \mathrm{~A} / \mathrm{min}$. respectively.

The XPS spectra, Figure 3.14 indicate the presence of $\mathrm{Cu}_{2} \mathrm{O}$ for Virgin copper wafers, while cleaned wafers show both $\mathrm{Cu}_{2} \mathrm{O}$ and $\mathrm{Cu}^{0}$. This suggests that the attack of $\mathrm{HF}$ on $\mathrm{Cu}_{2} \mathrm{O}$ may initiate the corrosion process, due to a favorable $\Delta \mathrm{G}(\approx-379$ $\mathrm{kJ} / \mathrm{mole}$ ) of the reaction. Two possible mechanisms explain the observation of $\mathrm{Cu}^{0}$ in the XPS data. First, stripping $\mathrm{Cu}_{2} \mathrm{O}$ by $\mathrm{HF}$ in the cleaning solution could expose the underlying $\mathrm{Cu}^{0}$, or second, the disproportionation of $\mathrm{Cu}^{+1}$ species (from the $\mathrm{HF}$ reaction) could give rise to $\mathrm{Cu}^{0}$. Both mechanisms predict that $\mathrm{Cu}$ (II) species should be present in the cleaning solution.

To test these models and to complement the electrochemical experiments, which indicated active dissolution of copper, we monitored the buildup of copper ions in the cleaning solution using ICP-MS. These curves can be fitted to pseudo first order kinetic process. The measured dissolution rates from the extrapolated slopes of the concentration vs. time curves at $\mathrm{t}=0$, yield values of $10 \mathrm{ppb} / \mathrm{min}$ in a non-deareated solution and $1 \mathrm{ppb} / \mathrm{min}$ in a deareated solution. The rate of copper dissolution is thus strongly dependent on the oxygen concentration. These findings are in qualitative agreement with electrochemically determined corrosion rate of $2 \mathrm{~A} / \mathrm{min}$ and 0.8 $\mathrm{A} \% \mathrm{~min}$, when corrected for the differences in geometric surface areas of samples.

The dissolution rate declines precipitously when a high $\mathrm{H}_{2} \mathrm{O}_{2}$ concentration is introduced in a non-deareated cleaning solution. The ICP-MS data analysis yields a very low copper dissolution rate of $0.15 \mathrm{ppb} / \mathrm{min}$. At such extremely low dissolution 
rates, on the timescale of experiment, lead to an apparent zeroth order (straight line) as opposed to the first order kinetics as seen in non-aerated/deareated solutions. Corrosion rate calculation of the polarization curve shown in Figure 3.7a, give a corrosion rate of $0.3 \mathrm{~A} / \mathrm{min}$, a value that is much lower than either the deareated or the non-deareated samples discussed before.

On a molecular level, the interface between copper and the cleaning solution differs in the presence of $\mathrm{H}_{2} \mathrm{O}_{2}$. The XPS results shown in Figure 3.15 reveal a replacement of $\mathrm{Cu}_{2} \mathrm{O}$ with $\mathrm{CuO}$ on the copper surface, perhaps due to oxidation by $\mathrm{H}_{2} \mathrm{O}_{2}$. Similar observations were made by Vazquez et al ${ }^{139}$ some time ago, who suggested that the peroxide reduction rate is significantly reduced in the presence of $\mathrm{CuO}$ in neutral/basic solutions. The slowdown in a cathodic reduction process would in turn reduce the anodic corrosion rate of copper. The lack of $\mathrm{Cu}(0)$ peak in the XPS spectrum indicates that a thicker $\mathrm{CuO}$ film covers the copper surface perhaps due to the high concentration of hydrogen peroxide used in this study. The shift of the corrosion potential to nobler values is consistent with peroxide reduction $\left(\mathrm{E}^{0}=1.776\right)$ being coupled to an anodic $\mathrm{Cu}$ oxidation, supplanting the normal cathodic oxygen reduction $\left(\mathrm{E}^{0}=1.229\right)$ mechanism. At the observed OCP in the peroxide containing solution, the Pourbaix diagram for copper would predict a stability and possible passivity conferred by $\mathrm{CuO}$. The XPS data in Figure 3.15 shows that $\mathrm{CuO}$ is only observed in peroxide containing solution thereby confirming the prediction of Pourbaix diagram. Thus, an interfacial $\mathrm{CuO}$ may provide an effective corrosion/dissolution barrier in the cleaning solution containing peroxide. An 
alternate mechanism of surface passivation ${ }^{140}$ in the cleaning solution containing oxidizer $\mathrm{H}_{2} \mathrm{O}_{2}$ could be due to its higher oxidizer concentrations compared to the experiments involving dissolved $\mathrm{O}_{2}$. Therefore, in summary, de-aeration/use of $\mathrm{H}_{2} \mathrm{O}_{2}$ can provide a successful strategy for cleaning formulation which protects the interconnect material.

These observations could be rationalized in terms of the following set of chemical reactions:

$$
\begin{array}{lc}
2 \mathrm{Cu}+1 / 2 \mathrm{O}_{2} \rightarrow \mathrm{Cu}_{2} \mathrm{O} & \Delta_{\mathrm{f}} \mathrm{G}=-146 \\
\mathrm{Cu}_{2} \mathrm{O}+2 \mathrm{HF} \rightarrow \mathrm{Cu}_{2} \mathrm{~F}_{2}+\mathrm{H}_{2} \mathrm{O} & \\
\mathrm{Cu}_{2} \mathrm{~F}_{2} \rightarrow \mathrm{CuF}_{2}+\mathrm{Cu}^{0} \quad \mathrm{E}=0.37 \mathrm{~V} & \Delta \mathrm{G}=\sim-10 \mathrm{Kcal} / \mathrm{mol} \\
\mathrm{Cu}+1 / 2 \mathrm{O}_{2} \rightarrow \mathrm{CuO} & \Delta_{\mathrm{f}} \mathrm{G}=-129.7 \\
\mathrm{CuO}+2 \mathrm{HF} \rightarrow \mathrm{CuF}_{2}+\mathrm{H}_{2} \mathrm{O} &
\end{array}
$$

Reactions 1-4 explain why the dissolution rate is oxygen dependant. The second reaction postulates formation of $\mathrm{Cu}^{1+}$ species in solution through the attack of $\mathrm{HF}$ on $\mathrm{Cu}_{2} \mathrm{O}$, the third reaction describes the disproportionation reaction (eq. 3) that causes immediate deposition of $\mathrm{Cu}$ and creates $\mathrm{Cu}^{2+}$ species. In the presence of $\mathrm{H}_{2} \mathrm{O}_{2}$, presumably surface cuprous oxide is oxidized to $\mathrm{CuO}$ or $\mathrm{Cu}$ is directly oxidized to cupric oxide which in turn is, slowly attacked by HF to form cupric fluoride (eq. 4, 5) which in solution can't disproportionate. Thus $\mathrm{H}_{2} \mathrm{O}_{2}$ may be help reduce copper deposition by quenching reaction 3 .

Literature data indicates that in $\mathrm{HF}$ based chemistry, $\mathrm{CuF}_{3}^{-}$ions are predominant species $^{141}$. Therefore, other possible reactions shown in table 3.1 for the 
formation of dissolution products in the dilute $\mathrm{EG} 3 / \mathrm{Cu}$ system were developed. Directions of those reactions were determined by calculating changes in standard Gibbs free energy $\left(\Delta \mathrm{G}^{\mathrm{o}}\right)$. However, the mechanism proposed for the deposition of copper (eqn. 9) are not relevant since the exposed surface of the wafer is not Si but its SiOx.

Table 3.1 Reactions showing $\mathrm{CuF}_{3}^{-}$formation and possible $\mathrm{Cu}$ deposition mechanism.

$$
\begin{aligned}
& \mathrm{Cu}^{2+}+3 \mathrm{~F}^{-} \rightarrow \mathrm{CuF}_{3}^{-} \\
& \mathrm{Cu}+3 \mathrm{HF}+1 / 2 \mathrm{O}_{2} \rightarrow \mathrm{HCuF}_{3}+\mathrm{H}_{2} \mathrm{O} \\
& \mathrm{Cu}+2 \mathrm{H}^{+}+3 \mathrm{~F}^{-} \rightarrow \mathrm{CuF}_{3}^{-}+\mathrm{H}_{2} \mathrm{O} \quad \Delta \mathrm{G}^{\circ}=-188.5 \mathrm{KJ} / \mathrm{mole}
\end{aligned}
$$

Mechanisms of $\mathrm{Cu}$ deposition:

$$
\mathrm{Si}+2 \mathrm{CuF}_{3}^{-} \rightarrow \mathrm{Cu}+\mathrm{SiF}_{6}^{-} \quad \Delta \mathrm{G}^{\mathrm{o}}=-624 \mathrm{KJ} / \mathrm{mole}
$$

\subsubsection{Factors Effect Thin Films Copper Corrosion}

Copper corrosion studies have been undertaken for decades. Most of the research up until recently was done utilizing bulk copper. The focus of this work was to investigate the corrosion behaviors of copper thin films. The corrosion behaviors of bulk copper and the techniques used to determine corrosion rates are not fully consistent with copper thin films. Copper thin films may behave significantly different due to thin film characteristics such as the crystallographic orientation and grain boundary volume, which are induced by stress and strain in the film, which is influenced by the deposition method.

Furthermore, with respect to dissolution rate, and dissolution mechanisms, the behaviors of blanket copper thin film wafers may not reflect strongly the behaviors of 
a patterned wafer during processing. The corrosion behaviors of dual damascene copper interconnect structures is governed by a combination of physical and chemical factors that are influential on the type of copper corrosion. These factors are summarized in the schematics given in Figure 3.17.

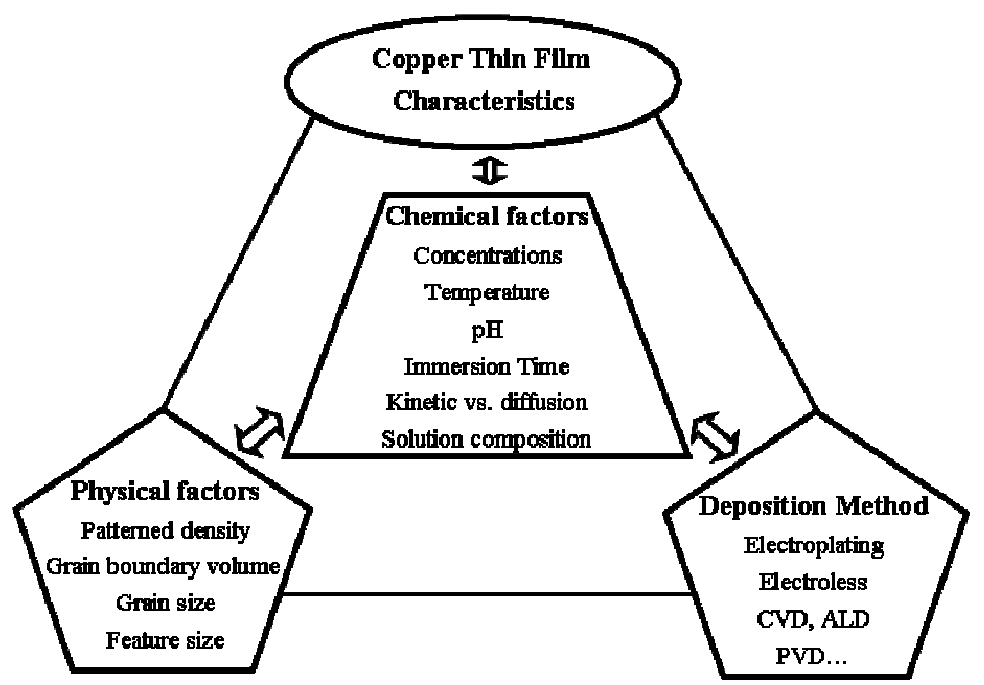

Figure 3.17 Factors influencing copper interconnects corrosion.

\subsubsection{Copper Corrosion Modes in Interconnects Structures}

The various potential modes or types of corrosion involved in an interconnect damascene structure are illustrated in Figure 3.18. Corrosion mechanisms and behaviors can become significantly more complex when considering the underlying interdependency of the aforementioned factors. For example, optimizing the deposition process of copper to achieve maximum conformality and uniformity may impact the corrosion resistance performance of the copper interconnect system. 


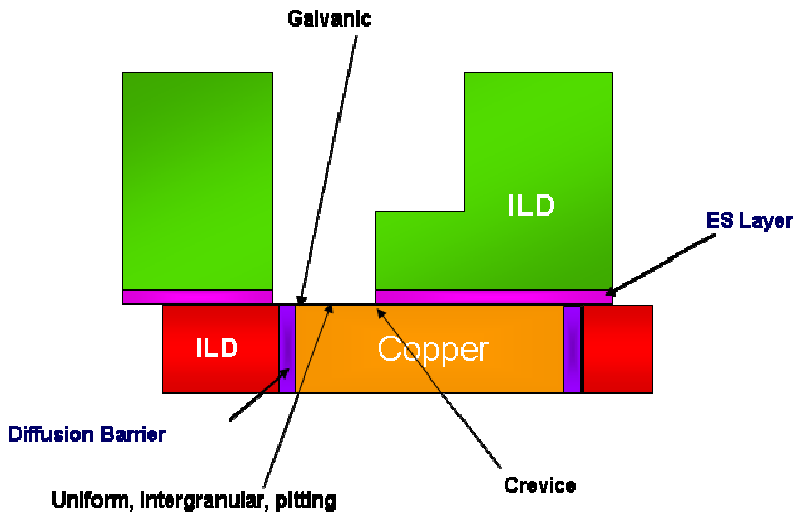

Figure 3.18 Modes of $\mathrm{Cu}$ corrosions that can take place in copper damascene structures.

\subsubsection{Copper Precipitation/Deposition Mechanisms}

It is conceivable that more than one mechanism is responsible for copper precipitation/deposition onto the ILD surface as presented in Figure 3.3. The occurrence of more than one mechanism at different regions of the processed wafer is feasible. The mechanism at which copper deposition/precipitation occurs can be attributed to factors such as surface properties, geometry of structures exposed, patterning density, etc... In addition to these factors, a series of radiochemical tracer studies by $\operatorname{Kern}^{142}$ suggested that the metal depositions on silicon surfaces depend on the immersion time, temperature and chemical compositions of the etching solutions. A number of plausible mechanisms may explain the phenomenon of copper deposition/precipitation onto the wafer surface. The source of copper can be attributed to precipitation of copper complexes from the cleaning solution, Plasma etch creates residue on the surface and Electroless deposition of copper $\mathrm{Cu}^{++} \rightarrow \mathrm{Cu}^{0}$ in which copper can plate out of the solution during the wet cleaning and etching process. ${ }^{143,144,}$ $145,146,147,148$. Our studies imply that an electroless deposition involving the reduction of cupric ions can be avoided by having an oxidizing species, such as $\mathrm{H}_{2} \mathrm{O}_{2}$ present in 
the cleaning solution provided it is compatible with other solution-exposed interfaces on the wafer. An additional source of copper deposits on wafer surface may arise from surface mediated nucleation and growth of copper complexes.

It is quite clear that in order to prevent the formation of copper rich residue on the ILD surface, copper corrosion must be prevented. Maintaining low copper concentrations allows maintaining these species below their solubility limits. Similarly, the harmful role of metallic contaminants in creating undesirable deposits is well established for bare silicon wafers ${ }^{149,150,151,152,153,154,155,156}$ in support of frontend-of the-line (FEOL) processing. This present study underscores analogous effects of the wafer surface contamination due to products of $\mathrm{Cu}$ interconnect corrosion in BEOL.

One of the limitations of the present study is that it utilized blanket (nonpatterned) wafers and not the copper damascene patterned wafers. It is known that the nanoscaled patterns may exhibit higher corrosion rates ${ }^{157}$ since the dissolution rates are expected to be dependent on a number of physical factors and film characteristics such as curvature (Kelvin effect), grain size, texture, geometry, and microstructure ${ }^{158}$, 159. Furthermore, with ever decreasing size of the features on the wafer surfaces, these features provide sites for heterogeneous nucleation. Even with respect to this enhanced deposition mechanism, reducing solution borne copper concentration, lowers the degree of supersaturation and hence the probability of nucleation events.

In addition to deareation and the use of $\mathrm{H}_{2} \mathrm{O}_{2}$, one may contemplate using corrosion inhibitors such as benzotriazole (BTA) to passivate the copper surface and 
prevent copper dissolution. We have established through ICP-MS based dissolution studies shown in Figure 3.11, that such corrosion inhibitors indeed reduce the copper dissolution rates. However, the mechanism for the dissolution suppression appears to be different from that observed in the peroxide containing solution. When the corrosion inhibitor are used the copper surface displays $\mathrm{Cu}(\mathrm{I})$ oxide (as shown in Figure 3.15), and presumably, the inhibitor film between copper surface and cleaning solution provides the corrosion resistance. Nevertheless, the introduction of such corrosion inhibitors in the cleaning solution could potentially lead to carbon deposits at subsequent processing stages. 


\section{A Kinetic Study of Copper Thin Film Dissolution}

Thin film copper dissolution and reaction kinetics were investigated by monitoring $\mathrm{Cu}^{+2}$ employing ICP-MS and oxidation states of copper on $\mathrm{Si}$ wafer surface by XPS. A kinetic scheme involving cathodic reduction of oxygen and anodic oxidation of $\mathrm{Cu}^{0}$ and $\mathrm{Cu}^{+1}$ is proposed that is consistent with experimentally determined reaction kinetic orders.

To probe further how the oxidizing agents such as oxygen can affect the corrosion process, time dependent $\mathrm{Cu}^{+2}$ concentration was investigated as shown in Figure 3.19. Here, an initial increase in the copper concentration was followed by significant slower rates of dissolution at later times. This trend was quasi-universal regardless of the oxygen concentrations and it is better discerned on a log scale, as shown in Figure 3.20. It should be noted that the concentration of $\mathrm{Cu}^{+2}$ at longer times is dependent on the dissolved oxygen concentration. Therefore, the ICP-MS measurements of copper concentration were supplanted with oxygen concentration as a function of time and the number of wafers. This data appears in Figure 3.21. The objective was to probe whether a steady state concentration of $\mathrm{O}_{2}$ [i.e., $\mathrm{d}\left[\mathrm{O}_{2}\right] / \mathrm{dt}=0$ ] is reached in the cleaning solution. Figure 3.21 presents the results for cleaning solutions that were isolated from the open air. The results show that within 300 minutes, a timescale comparable to the initial surge in copper dissolution shown in Figures 3.19 and 3.20 , the corresponding oxygen concentration in the cleaning solution decreases and reaches a steady state. In the same solution, two more wafers were added at $\mathrm{t}=8700$ minutes and the system was again isolated from air. Resulting 
decline in $\mathrm{O}_{2}$ concentration occurred on the similar timescale reaching a new lower steady state of oxygen concentration $(\approx 1.5 \mathrm{ppm})$. It is clear, therefore, that the corrosion process involves oxygen participation (see later).

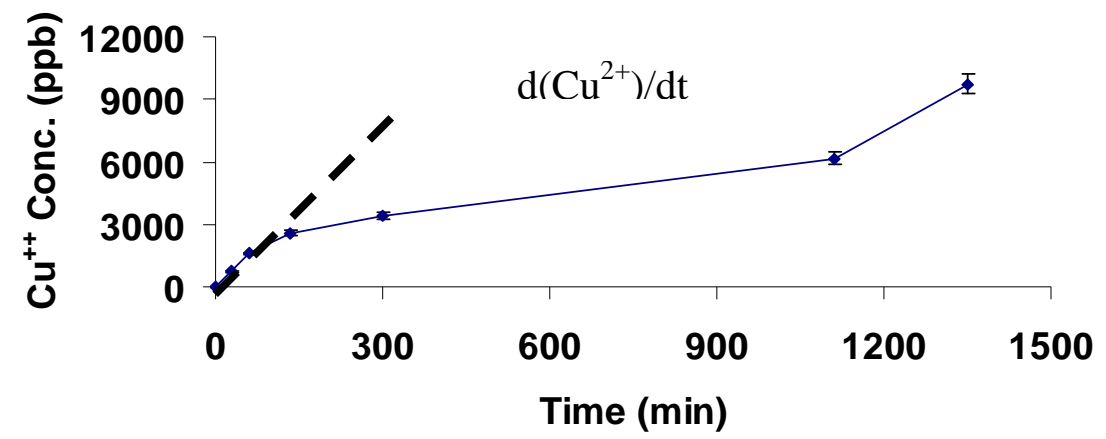

Figure 3.19 $\mathrm{Cu}$ dissolution in aerated clean solution as a function of time. The DO concentration was $8.9 \mathrm{mg} / \mathrm{L}$.

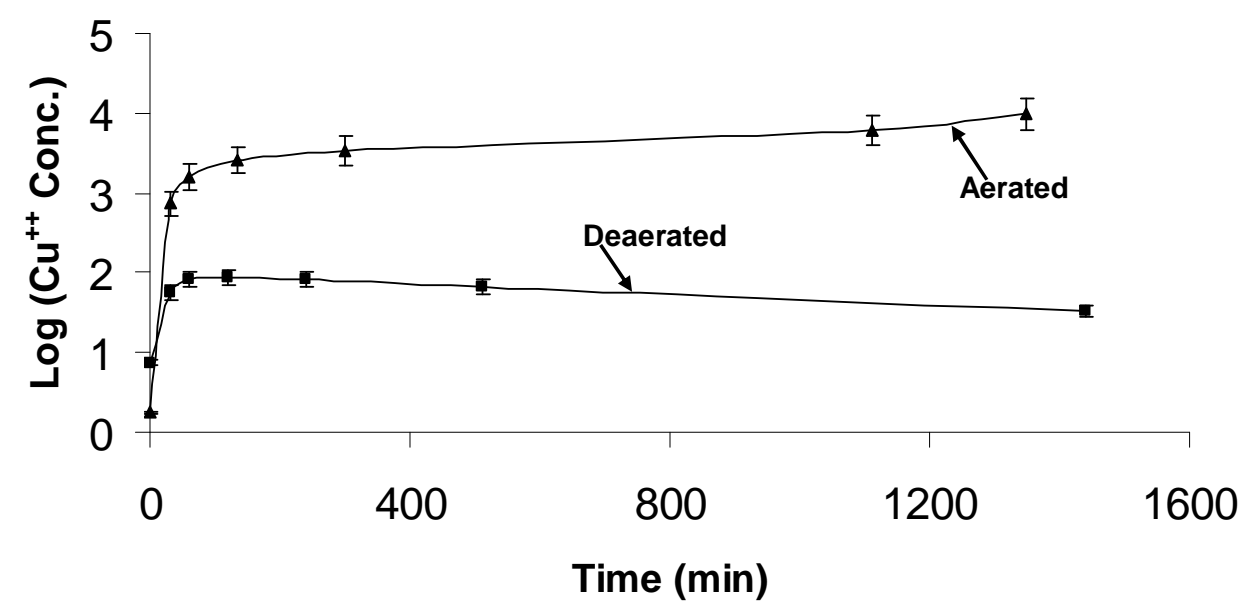

Figure 3.20 $\mathrm{Cu}$ dissolution in aerated and deareated clean solution as a function of time. The DO concentrations were 8.9 and $0.6 \mathrm{mg} / \mathrm{L}$ in the aerated and deareated solutions, respectively. 


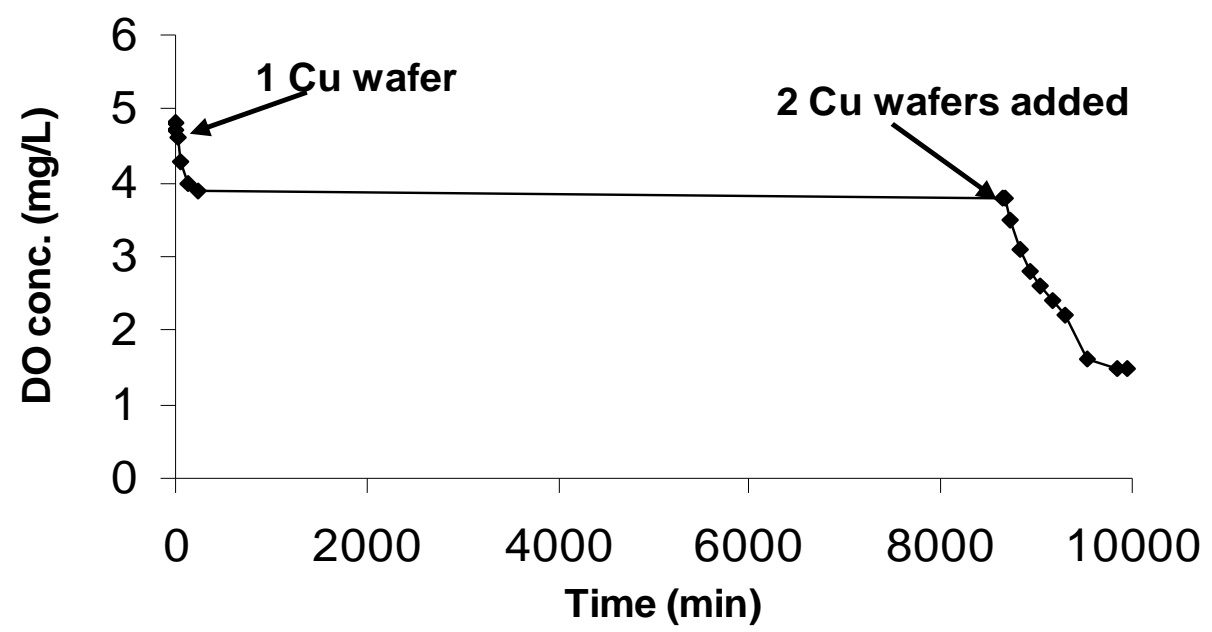

Figure 3.21 DO Concentration in the clean solution in a sealed container as a function of time and $\mathrm{Cu}$ surface area.

The essential component of the cleaning solution is HF. It can attack exposed oxide surfaces of copper and silicon thereby functioning as a cleaning agent provided the duration of cleaning is precisely controlled. Thermodynamically, the free energy of formation for $\mathrm{CuF}_{2}$ from $\mathrm{Cu}^{0}$ or $\mathrm{Cu}^{+}$is negative. However, in our prior electrochemical studies, it was noted that electrochemically formed $\mathrm{CuO}$ film confers a degree of passivity to the copper film; while aerated and non-deareated solutions generally corrode at appreciable rate.

A simple electrochemical model of the dissolution process is proposed in Figure 3.22. It depicts cathodic reduction of oxygen coupled with anodic oxidation of $\mathrm{Cu}^{0}$ or $\mathrm{Cu}^{+1}$ that lead to the formation of $\mathrm{Cu}^{+2}$ species in the cleaning solution. More specifically: 
Cathodic reduction

$$
2 \mathrm{H}^{+}+2 e+1 / 2 \mathrm{O}_{2} \rightarrow \mathrm{H}_{2} \mathrm{O}
$$

Anodic oxidation

$$
\begin{aligned}
2 \mathrm{Cu}^{0} & \rightarrow 2 \mathrm{Cu}^{+}+2 e \\
2 \mathrm{Cu}^{+} & \rightarrow 2 \mathrm{Cu} u^{++}+2 e \\
\text { or } \mathrm{Cu}^{0} & \rightarrow \mathrm{Cu}^{++}+2 e
\end{aligned}
$$

Net reactions

$$
\begin{aligned}
& 2 \mathrm{Cu}^{+}+2 \mathrm{H}^{+}+\frac{1}{2} \mathrm{O}_{2} \rightarrow 2 \mathrm{Cu}^{++}+\mathrm{H}_{2} \mathrm{O} \\
& \mathrm{Cu}^{0}+2 \mathrm{H}^{+}+\frac{1}{2} \mathrm{O}_{2} \rightarrow \mathrm{Cu}^{++}+\mathrm{H}_{2} \mathrm{O}
\end{aligned}
$$

Given that our XPS data always shows presence of $\mathrm{Cu}^{+1}$ species, see Figure 3.14, oxidation of $\mathrm{Cu}^{0} \rightarrow \mathrm{Cu}^{+1}$ through formation of cuprous oxide might be the rate limiting process. Thermodynamically the following reaction steps are energetically feasible.

$$
\begin{aligned}
& \text { Without } \mathrm{O}_{2}: \mathrm{Cu}_{2} \mathrm{O}+2 \mathrm{HF} \rightarrow \mathrm{Cu}_{2} \mathrm{~F}_{2}+\mathrm{H}_{2} \mathrm{O} \rightarrow \mathrm{Cu}^{0}+\mathrm{CuF}_{2}+\mathrm{H}_{2} \mathrm{O} \\
& \text { With } \mathrm{O}_{2}: 2 \mathrm{Cu}^{0}+1 / 2 \mathrm{O}_{2}+\mathrm{H}_{2} \mathrm{O} \rightarrow \mathrm{Cu}_{2}(\mathrm{OH})_{2} \\
& \mathrm{Cu}_{2} \mathrm{~F}_{2}+2 \mathrm{HF}+\frac{1 / 2}{2} \mathrm{O}_{2} \rightarrow 2 \mathrm{CuF}_{2}+\mathrm{H}_{2} \mathrm{O} \\
& \quad \mathrm{Cu}+\mathrm{CuF}_{2} \\
& \mathrm{Cu}_{2} \mathrm{~F}_{2]}^{\mathrm{Z}} \\
& \quad+2 \mathrm{HF}+1 / 2 \mathrm{O}_{2} \rightarrow 2 \mathrm{CuF}_{2}+\mathrm{H}_{2} \mathrm{O}
\end{aligned}
$$

The disproportionation of $\mathrm{Cu}^{+}$species is a well known effect observed with other copper halides, although it has never been firmly established in the case of $\mathrm{Cu}_{2} \mathrm{~F}_{2}$. Disproportionation is consistent with the observed copper rich residues (Figure 3.3) on the ILD surfaces, although it does not prove its occurrence. In the above reaction 
schemes we emphasize the role of $\mathrm{O}_{2}$ in corrosion process through the formation of $\mathrm{Cu}_{2}(\mathrm{OH})_{2}$ and its decomposition in the presence of $\mathrm{HF}$.

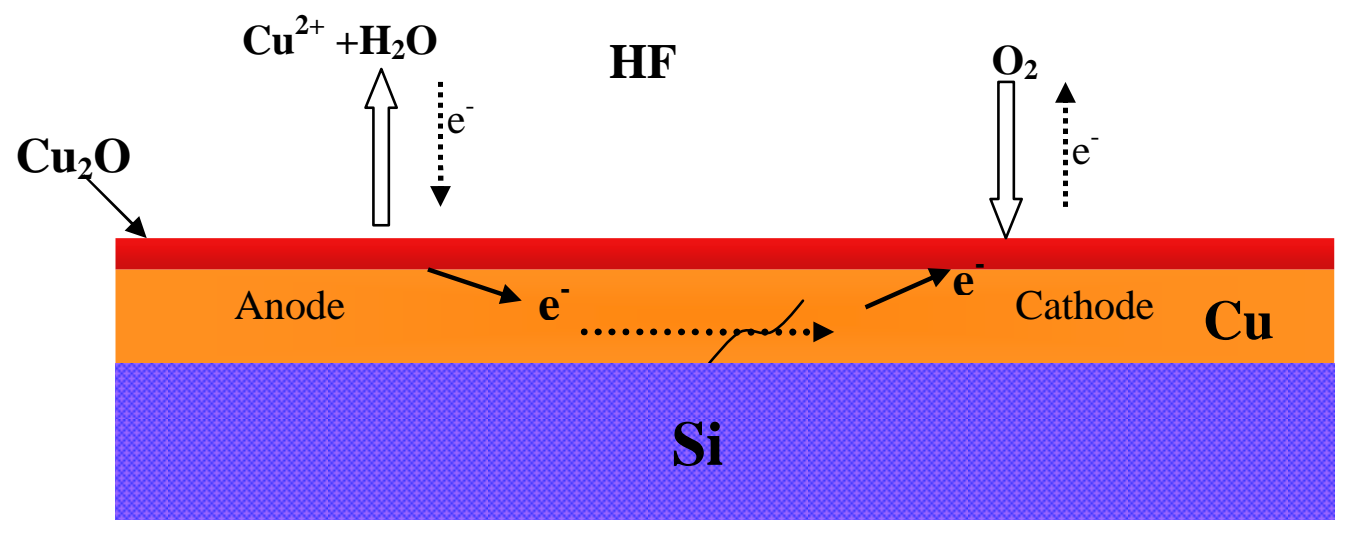

Figure 3.22 A simple electrochemical model of the dissolution process showing the cathodic reduction of oxygen coupled with anodic oxidation of $\mathrm{Cu}^{0}$ or $\mathrm{Cu}^{+1}$ that lead to the formation of $\mathrm{Cu}^{2+}$ species in the cleaning solution.

To further develop molecular mechanism of kinetics of copper dissolution we focus on the short-time behavior. This is directly relevant for semiconductor processing, as typically wafers are expected to be cleaned in less than 5 minutes. To study the kinetics in this time region, a method of initial rates was employed where the $\mathrm{d}\left[\mathrm{Cu}^{+2}\right] / \mathrm{dt}$ was determined by drawing a tangent to copper concentration vs. $\mathrm{t}$ curve in the limit $t \rightarrow 0$. This is shown schematically by the dotted line in Figure 3.19. Under these conditions a general rate equation for appearance of $\mathrm{Cu}^{+2}$ species in the cleaning solution can be written as:

$$
\frac{d\left[C u^{+2}\right]}{d t}=k\left[O_{2}^{S \ln }\right]^{n}[H F]^{m}
$$


Here $n$ and $m$ are the reaction orders with respect to dissolved oxygen $\left(\mathrm{O}_{2}{ }^{\mathrm{Sln}}\right)$ and HF concentration respectively. Figure 3.23a depicts $\ln -\ln$ plot of the copper dissolution rate versus the $\mathrm{O}_{2}{ }^{\text {Sln }}$ concentration in a fixed $\mathrm{HF}$ concentration (note dissolution rates are in $\mathrm{ppb} / \mathrm{minute}$, while concentration of $\mathrm{HF}$ is in high 100's of ppm range). Similarly for fixed oxygen concentration $(<10 \mathrm{ppm}$ range) in well stirred cleaning solutions, the dissolution rate was determined as a function of HF concentration, Figure 3.23b. The measured values of $\mathrm{n} \approx \mathrm{m} \approx 1.0 \pm 0.1$. Also plots of $\ln \left(\mathrm{O}_{2}-\mathrm{O}_{2}{ }^{\mathrm{SS}}\right)\left(\mathrm{O}_{2}{ }^{\mathrm{SS}}\right.$ denotes a steady state concentration of $\mathrm{O}_{2}{ }^{\mathrm{Sln}}$ ) versus time were linear (not shown) implying a first order rate process for the decrease in $\mathrm{O}_{2}$ concentration presented in Figure 3.21.

a) kinetic order with respect to Oxygen $0.9+-0.1$

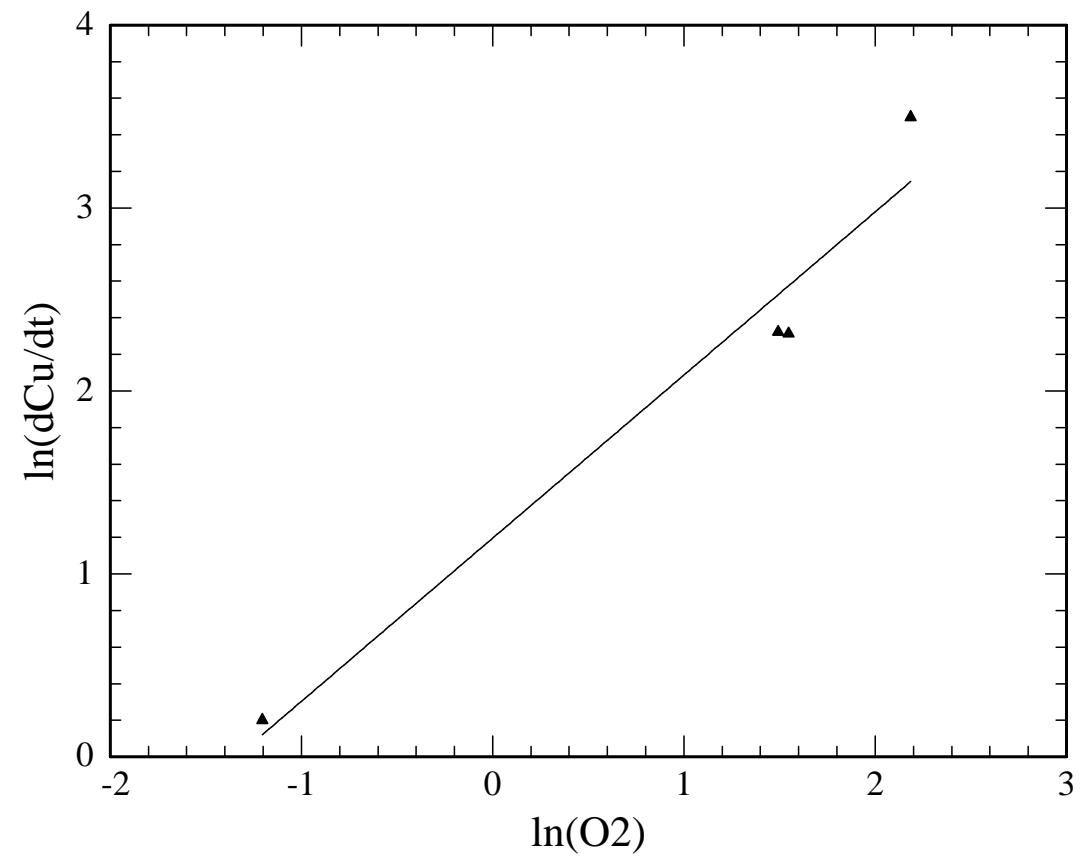


b)

Kinetic order with respect to HF 0.9+-0.1

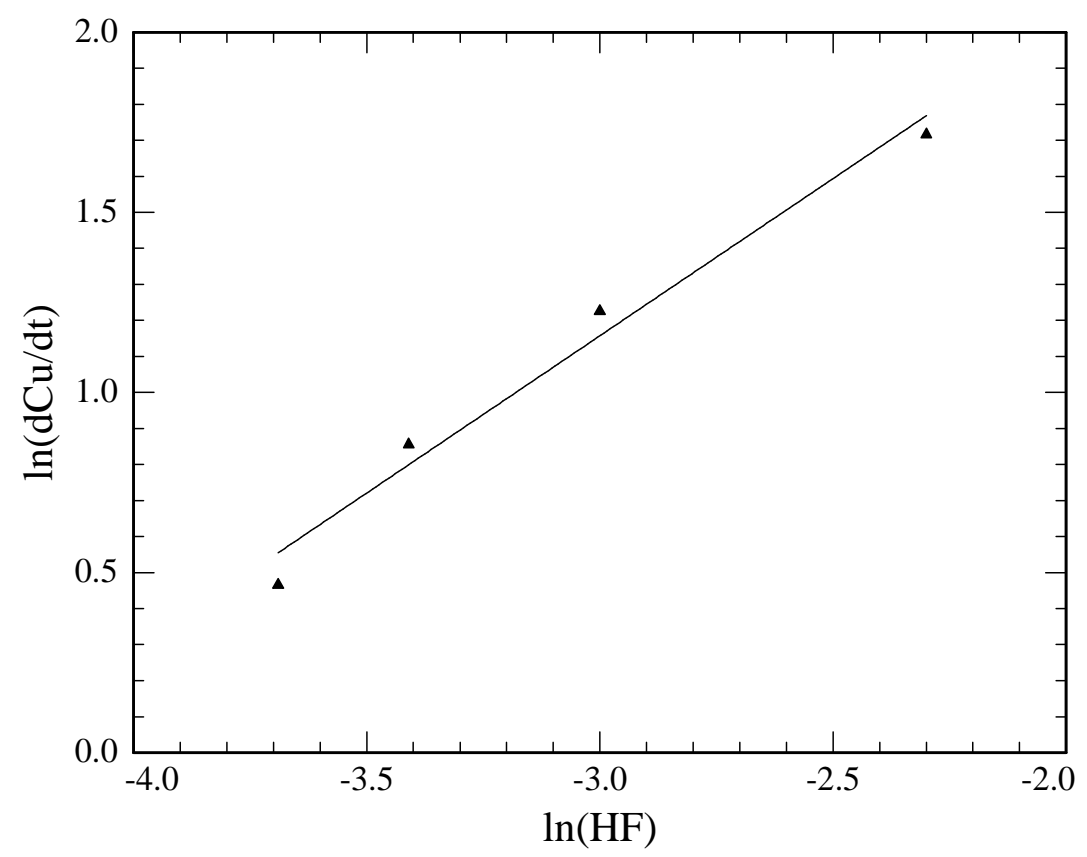

Figure 3.23 $\mathrm{Cu}$ dissolution order kinetics with respect to DO and HF concentrations in (a) and (b) respectively.

A plausible kinetic scheme is presented below:

$$
\begin{aligned}
& \mathrm{O}_{2}^{s \ln } € \quad \mathrm{O}_{2}^{a d s} \quad k_{0}^{e q} \\
& \mathrm{O}_{2}^{a d s} \stackrel{k_{1}}{\longrightarrow} 2 \mathrm{O}^{a d s} \\
& 2 \mathrm{Cu}+\mathrm{O}^{a d s} \mathrm{H}_{2} \mathrm{O} \underset{k_{-2}}{\stackrel{k_{2}}{C^{2 d}} u_{2}(\mathrm{OH})_{2} \quad k_{2}^{e q}} \\
& \mathrm{Cu}(\mathrm{OH})_{2}+\mathrm{HF} \stackrel{k_{3}}{\longrightarrow} \mathrm{Cu}(\mathrm{OH}) \mathrm{CuF}+\mathrm{H}_{2} \mathrm{O} \\
& \mathrm{Cu}(\mathrm{OH}) \mathrm{CuF}+\mathrm{HF} \stackrel{k_{4}}{\longrightarrow} \mathrm{Cu}_{2} F_{2}+\mathrm{H}_{2} \mathrm{O} \\
& \mathrm{Cu} \mathrm{F}_{2} \stackrel{k_{5}}{\longrightarrow} \mathrm{Cu}^{0}+\mathrm{CuF}_{2}
\end{aligned}
$$


The corresponding kinetic analysis is presented in appendix A. It provides the following simple rate expression for copper dissolution: $\frac{d\left[\mathrm{Cu}^{+2}\right]}{d t}=k_{3}\left[\mathrm{Cu}_{2}(\mathrm{OH})_{2}\right][H F]=\frac{2 k_{0}^{e q} k_{1} k_{2}^{e q} k_{3}\left[O_{2}^{s \ln }\right][H F]}{\left(k_{2}-k_{-2} k_{2}^{e q}\right)}$

In this analysis, the kinetic rate of adsorbed $\mathrm{O}_{2}$ dissociation as well as the rate of $\mathrm{Cu}_{2}(\mathrm{OH})_{2}$ formation provides the plausible rate determining steps. This expression is consistent with observed rate law, although we have not detected the postulated intermediates such as $\mathrm{Cu}_{2} \mathrm{~F}_{2}$ or $\mathrm{Cu}(\mathrm{OH}) \mathrm{CuF}$ in our experimental work. In view of these considerations, we have not further modeled the detailed kinetics that could includes the second oxidation step involving oxygen for the $\mathrm{Cu}^{+}$species (see eqn. 3.11 above), as it would entail postulating additional intermediates (e.g. $\mathrm{H}_{2} \mathrm{Cu}_{2} \mathrm{~F}_{4}$ )etc. Nonetheless, if the branching ratio of $\mathrm{Cu}_{2} \mathrm{~F}_{2}$ favors disproportionation then the kinetic analysis presented above becomes applicable to mechanistic model of copper corrosion and deposition of undesired copper-rich residues on the ILD surfaces in the HF containing cleaning solution.

Here, the findings of this work were compared with an early study of copper electrolyte interface by Chan et $\mathrm{al}^{160}$. In this classic work it was noted that surface oxide $\mathrm{Cu}_{2} \mathrm{O}$ formation is detected in basic/neutral $\mathrm{pH}$ conditions while in acidic conditions formation $\mathrm{Cu}-\mathrm{X}$ species is more commonly observed ${ }^{161,162,163,164}$. Chan et al observed that even in acidic conditions adsorbed oxygen species as opposed to oxide formation was detectable in surface enhanced Raman studies. However, near neutral $\mathrm{pH}$ both formation of oxide as well as halides of $\mathrm{Cu}^{+1}$ were suggested. In this 
scenario copper oxidation is driven by halides. The following sequences can be suggested for anodic oxidation of copper by fluoride ions based on their work.

$$
\begin{aligned}
& 2 \mathrm{Cu}+2 \mathrm{~F}^{-} \stackrel{k_{2}}{\rightarrow} \mathrm{Cu}_{2} \mathrm{~F}_{2}+2 e \\
& \mathrm{Cu}_{2} \mathrm{~F}_{2}-\stackrel{k_{5}}{\rightarrow} \mathrm{C} u^{0}+\mathrm{CuF} F_{2} \\
& \mathrm{O}^{\mathrm{Ads}}+2 \mathrm{H}^{+}+2 e \rightarrow \mathrm{H}_{2} \mathrm{O} \\
& 2 \mathrm{Cu}+2 \mathrm{HF} \stackrel{k_{2}}{\rightarrow} \mathrm{H}_{2} \mathrm{Cu}_{2} \mathrm{~F}_{2} \\
& \mathrm{H}_{2} \mathrm{Cu}_{2} \mathrm{~F}_{2}+\mathrm{O}^{\mathrm{Ads}} \stackrel{k_{3}}{\rightarrow} \mathrm{H}_{2} \mathrm{O}+\mathrm{CuF}_{2}+\mathrm{Cu}^{0}
\end{aligned}
$$

In this scheme copper oxidation is driven by $\mathrm{F}^{-}$attack and compensating oxygen reduction is postulated. This mechanism does not lead to first order kinetics with respect to $\mathrm{HF}$ and dissolved oxygen. Given that our cleaning solution exhibits a $\mathrm{pH}$ that is nearly neutral $\left(\mathrm{pH}=6.8\right.$ ), the formation of $\mathrm{Cu}_{2}(\mathrm{OH})_{2}$ (i.e., eqn. 3.13c) is expected to dominate the dissolution kinetics as was suggested in equation 3.13. This is also consistent weak passivation layer that observed in the potentiodynamic studies. Assuming that such layer is made of porous oxide film, the attack of HF as well as oxygen access to the underlying copper surface appears feasible. A more detailed verification of these models would require systematic studies that involve variation of $\mathrm{pH}$ of the cleaning solution to observe differing kinetic exponents $\mathrm{n}$ and $\mathrm{m}$ consistent with reaction sequences presented in equation 3.15. Such studies are beyond the scope of the present investigation.

\subsection{Summary}

Potentiodynamic polarization experiments carried out in the cleaning solution, show active, active-passive, passive, and transpassive regions. Use of organic 
corrosion inhibitors, deareated solutions and possible use of $\mathrm{H}_{2} \mathrm{O}_{2}$ provides an effective method to reduce corrosion rates and copper concentration in cleaning solution. $\mathrm{H}_{2} \mathrm{O}_{2}$ addition to the cleaning solution appears to be very beneficial by creating a $\mathrm{CuO}$ film that is resistant to attack by HF. The incorporation of $\mathrm{H}_{2} \mathrm{O}_{2}$ provides an additional advantage by enhancing the cleaning solution's ability in removing particles and residue. Furthermore, a simple kinetic model is proposed for copper dissolution during wet chemical etch process. It predicts a first order kinetics with respect to dissolved oxygen and HF, the two key ingredients involved in corrosion of copper. If dissolved oxygen is eliminated or if protective $\mathrm{CuO}$ is formed the rate of copper corrosion can be suppressed. 


\section{CHAPTER 4}

\section{Titanium Assisted Dual Damascene Patterning of Advanced Copper Interconnects and Selective Wet Etch Concepts}

\subsection{Introduction}

The focus in this chapter and consecutive chapters is restricted to a novel formulation designed to selectively strip patterned titanium thin film without impacting key interconnect/transistor materials. The concepts and learnings developed in chapter 3, particularly those pertaining to corrosion characterization and corrosion prevention of copper thin films are extended to investigate the corrosion behaviors of titanium and tungsten thin films and their relevance to future ULSI process technologies.

The chapter begins with a description of titanium assisted dual damascene patterning of copper interconnects process. The concepts of etch rate and wet etch selectivity and compatibility in the integration of titanium hard mask and its relevance to advanced logic process technology is then presented. Finally, the properties and corrosion behaviors of Ti and $\mathrm{W}$ are discussed. As previously pointed out, Ti and $\mathrm{W}$ are the metals of interest for the first layer of Ti assisted DD patterning interconnects. Therefore, the developed formulation must be capable to strip Ti while exhibiting no reactivity towards W. For upper interconnect layers, copper compatibility must be demonstrated by the wet etch chemical formulation. 
As discussed in details in chapter one, one of the many benefits associated with the incorporation of a metal hard-mask (MHM) in the integration of advanced logic process technology for dual damascene patterning of copper interconnects is to control via flare. The industrie's migration to low-K ILD materials imposed considerable difficulties due to their weak mechanical strength and the associated susceptibility to flare the top of the via or to cause deformation in the via profile during the etch process, as shown in Figure $4.1^{165}$. The via etch profile impacts reliability through its effect on the continuity and conformality of the metallic copper diffusion barrier to be deposited. Moreover, a MHM allows for a wide process window for etch rate control, permits for etching high aspect ration structures with robust profiles, protects the ILD material from damage induced by the plasma etch process, and acts as an interface between the ILD and photoresist during both the via and trench patterning steps. This is critical when polymer ILDs are used, since dry etch selectivity between photoresist and polymer ILD is difficult to attain. The etch strategy relies on consuming most, if not all of the photoresist during the via/or trench etch process. Under such circumstances, the presence of the hard-mask prevents the via and trench etch chemistry from attacking the polymer ILD in the masked regions of the wafer when the photoresist is consumed. This demand leads to the concept of cap layers, which may be used concurrently as hard-mask ${ }^{166}$. A dual hard-mask approach ${ }^{167,168,169}$, and triple hard-mask integration schemes ${ }^{170,171,172}$ are also proposed for patterning DD structures in polymer and hybrid ILDs. 


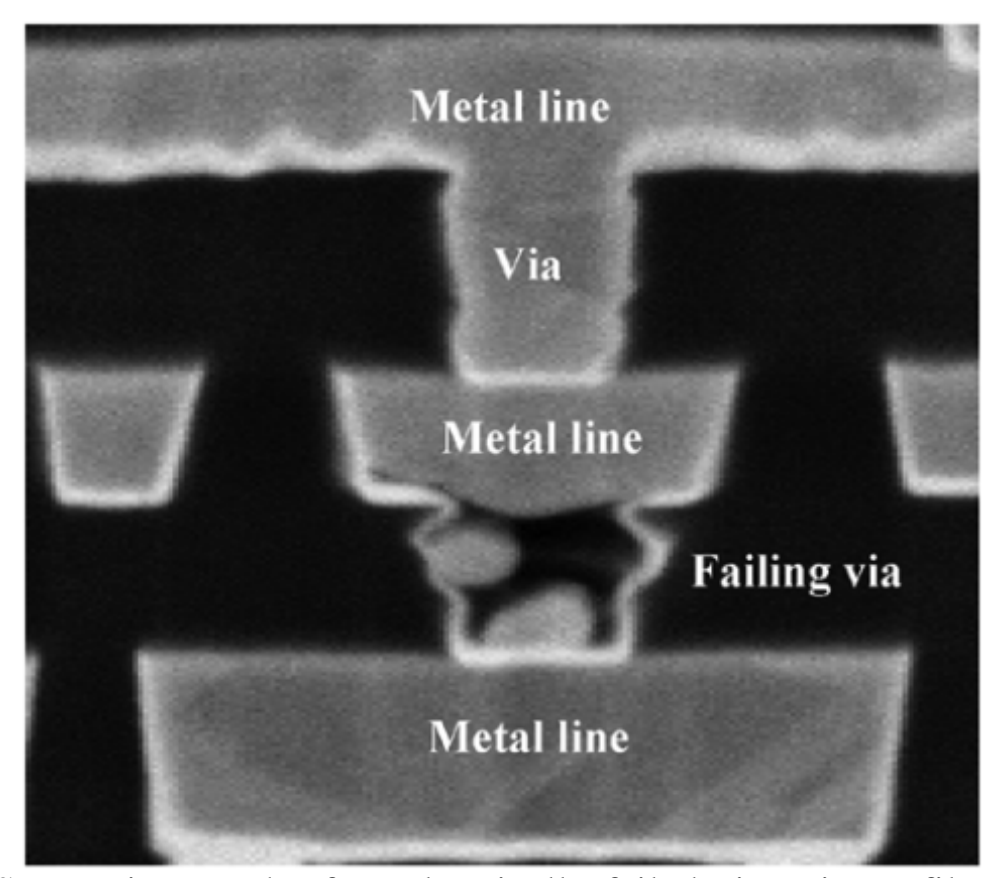

Figure 4.1 SEM micrograph of an electrically failed via. Via profile affects barrier deposition. Note that in addition to the degraded via profile, there is a high level of striation inside the 193-nm via.

To fully realize the aforementioned benefits of employing a hard-mask, a metallic hard-mask must be used. Conventional inorganic hard-masks (e.g., $\mathrm{SiO}_{2}$, SiC...) are inadequate advanced interconnect systems in the sub $50 \mathrm{~nm}$ and beyond, due to their low selectivity and severe faceting generated during the dielectric etch processes ${ }^{173}$. New metallic materials such as $\mathrm{Ti}$, TiN, TaN, etc..., have a different chemical nature than dielectric material allowing for better hard mask capabilities ${ }^{174}$ than conventional inorganic hard-masks. The work of this dissertation focuses on titanium HM. The overall advantage of employing MHM, enables further miniaturization which allows for the increase in component density. A visual illustration of the technology trend and miniaturizing is illustrated in Figure 4.2. 


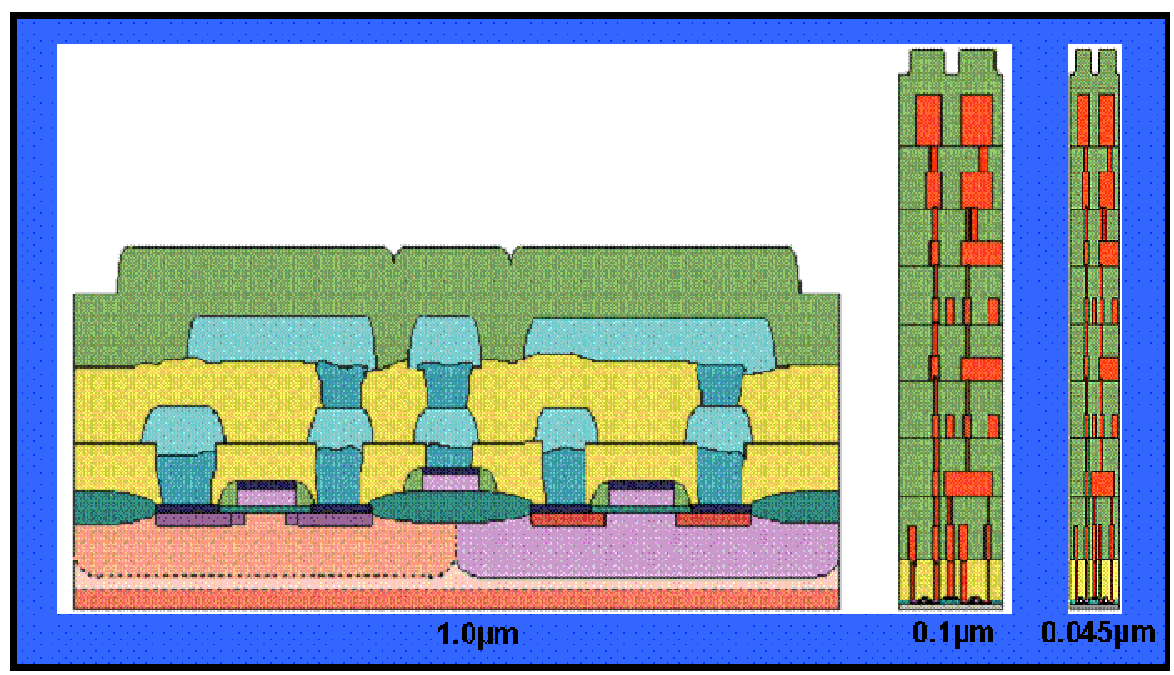

Figure 4.2 Schematic cross section of an IC showing the Technology miniaturizing trend $^{175}$.

\subsection{Titanium Assisted DD patterning of advanced copper interconnects}

The arrival of process technology in the sub $50 \mathrm{~nm}$ mandated the adoption of a titanium hard-mask to meet miniaturization requirements. Although the industry was united in its adoption of DD as the method of choice for manufacturing copper interconnects, several integration schemes and material stacks were proposed and implemented. The two most widely used are the trench-first and the via-first schemes. On the other hand, the material stack may contain multiple etch stop (ES) layers and/or hard masks (HM). This dissertation focuses on the via-first integration scheme with a single ES layer and a single metal hard-mask layer as this is the most cost effective approach in manufacturing high-performance microprocessors ${ }^{176,177,178}$.

The process flow of a simple, two transistors, and two-layer interconnect system involving the integration of titanium hard-mask is illustrated in Figure 4.3. In (a) The vias are patterned, in (b) The trenches and vias patterning is complete, (c) shows the photoresist and light absorbing material (also known as anti-reflective coating (ARC)) 
films are stripped, and the etch stop layer is etched at the bottom of the vias to expose the tungsten. Note the ES layer is a unique feature in the DD integration and patterning process. The primary function of this layer is to act as a diffusion barrier that inhibits copper diffusion into the ILD. It allows the film to seal and protect underlying copper from wet chemicals used during the patterning process and improves reliability. The ES layer also functions as a layer in which the via etch process terminates. This functionality is also needed to prevent etching into the ILD beyond desired via depth in the case of unlanded vias, where interconnect design rules allow for a via to partially land on the underlying metal layer, as illustrated in the Figure. In (d), the metal hard-mask and plasma etch polymer/residue are removed, and in (e), the copper diffusion barrier (DB) and copper are deposited in the vias and trenches.

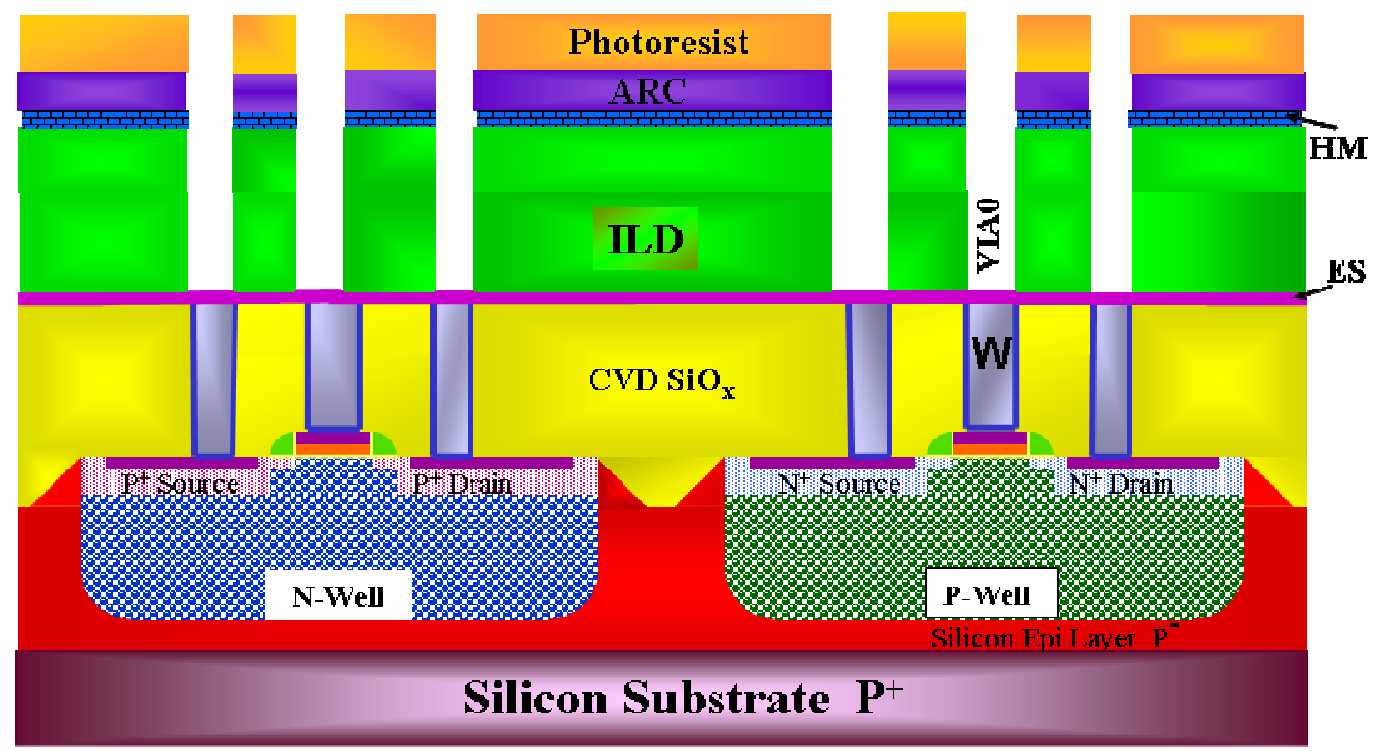

(a) 


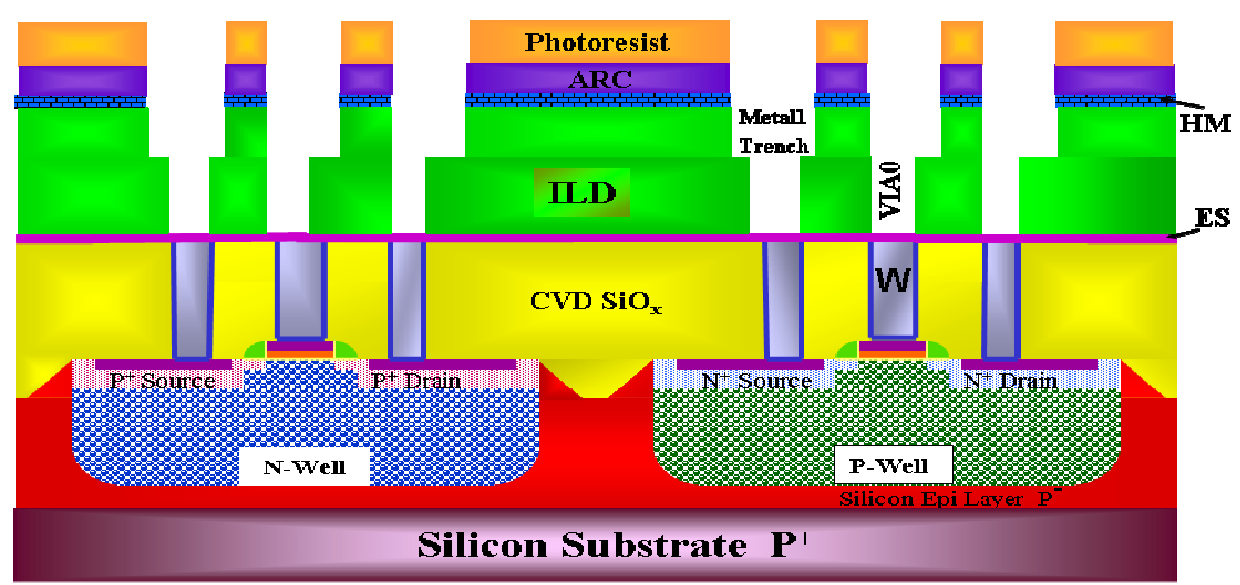

(b)

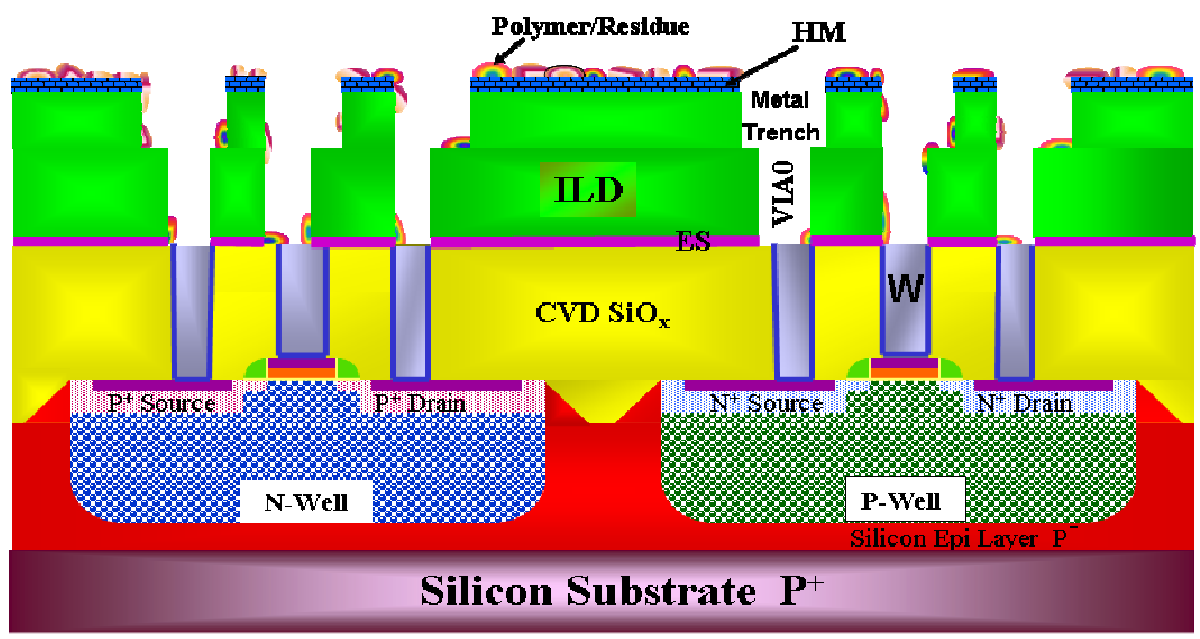

(c)

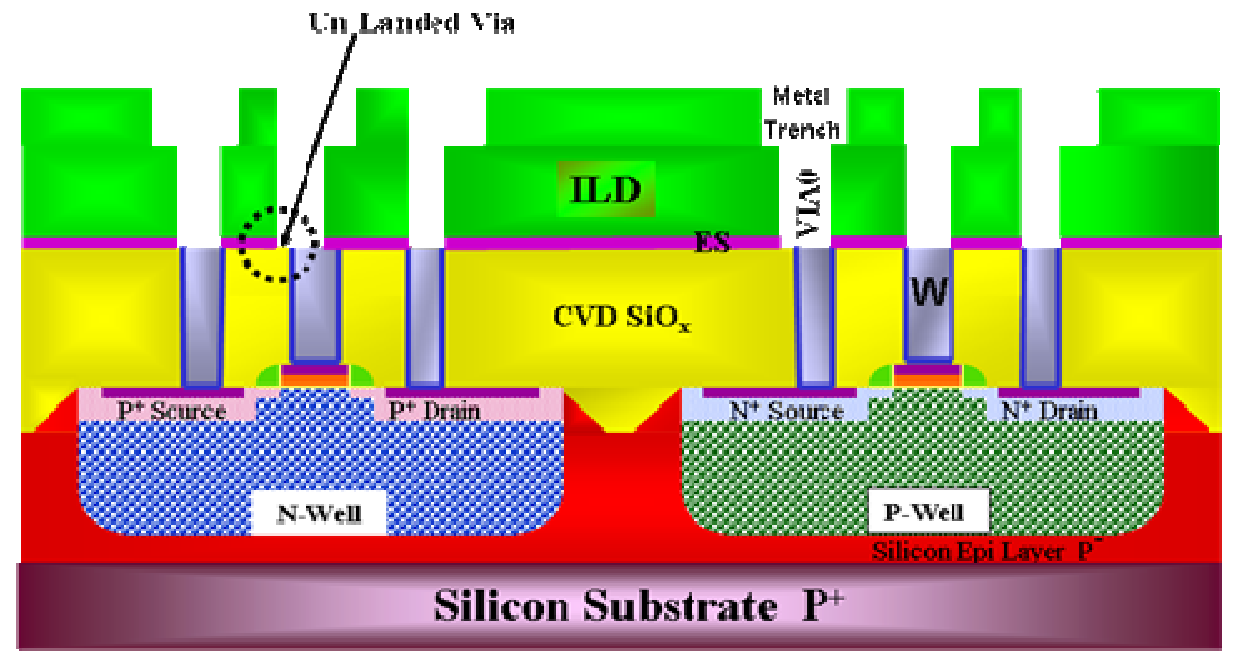

(d) 


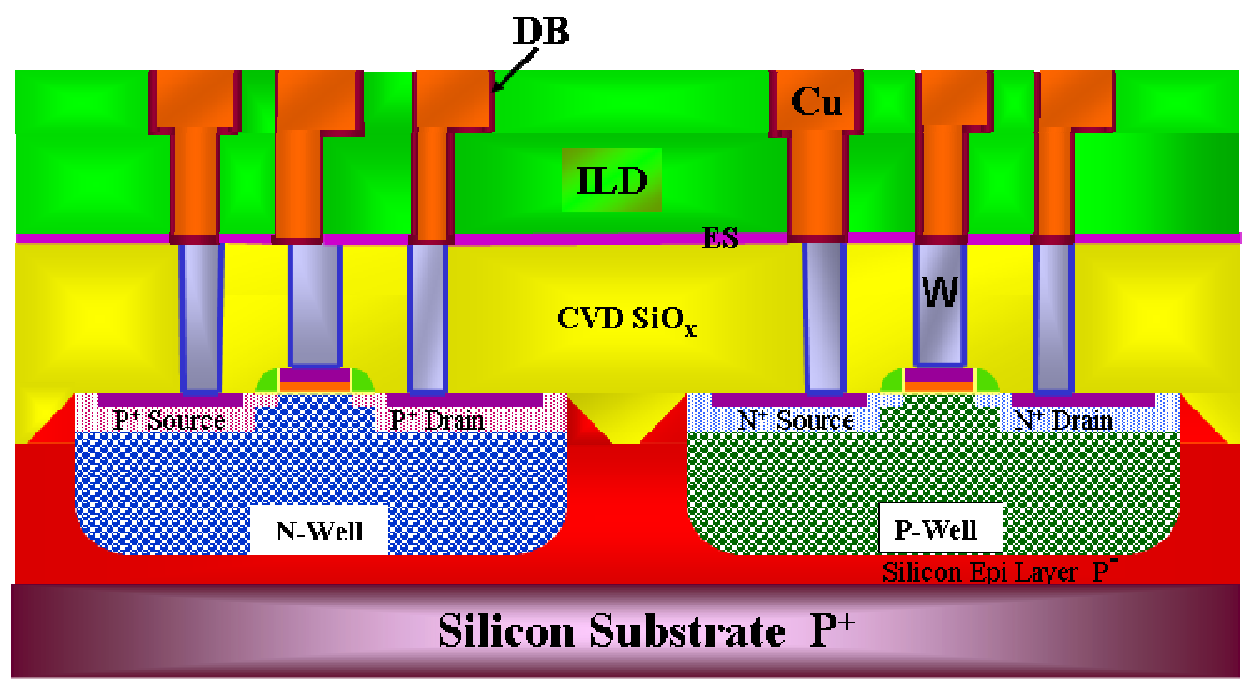

(e)

Figure 4.3 Schematic cross section of DD patterning steps involving a metal hardmask. (a) The via is patterned, (b) The trench is patterned, (c) photoresist and light absorbing material films are removed, and the etch stop layer is etched at the bottom of the vias exposing the $\mathrm{W}$, (d) The metal hard-mask and polymer/residue are removed, and (e) The copper diffusion barrier (DB) and copper are deposited in the vias and trenches.

\subsection{Basic Wet Etching Terminology}

In the IC manufacturing industry, a wet etch process refers to the employment of liquid etchants (chemical solutions) to remove an unwanted material such as a residue/polymer or a thin film from the surface of a wafer. Wet etching is isotropic or multidirectional, which indicates that the film or material is etched at an equal rate in all directions. In contrast, plasma etching is known to be anisotropic or unidirectional. The immersion and spray methods are the two most widely used wet etch process techniques in the fabrication of semiconductor devices. In the immersion method, the wafers are immersed in a tank that contains the chemical solution and is usually a batch process since several wafers are processed at the same time. On the other hand, the spray method is typically a single-wafer processing technique where the wafer is 
sprayed with the chemical solution while rotating. Key parameters defining the etching processes are discussed below.

\subsubsection{Etch Rate}

The etch rate for a material in a particular wet etch chemical solution is intuitively defined as how fast is a material or a film is removed from a surface. The etching rate is limited by two fundamental mechanisms, diffusion or kinetic (activation). The diffusion mechanism is reactant/product controlled and it is sensitive to agitation where the reactants are transported to the surface, surface reaction takes place, and products are transported from the surface. The activation limited scheme is dependent on surface reaction rate constant. The rate of etching is given by

$$
\text { Rate }=R_{o} \exp \left[-E_{A} / k_{B} T\right]
$$

Where $R_{0}$ is the rate constant and depends on reactant density, $E_{A}$ is the activation energy, $\mathrm{k}_{\mathrm{B}}$ is the Boltzmann's constant, and $\mathrm{T}$ is the temperature.

The etch rate of a certain material is dependent on the composition of the wet etch chemical composition, concentration, and temperature. Advantages and disadvantages of wet etching have been well defined ${ }^{179,180}$. Variation of etch rate with respect to age of the chemical solution and/or the age of the solution in the process bath, non uniform etching, and loading effects are some of the limitations that must be identified and characterized in order to achieve a successful wet etch process with stable etch rate. 


\subsubsection{Wet Etch Selectivity}

Selective wet etch chemical solutions are essential to device design and manufacturing of advanced ULSI technologies. The ability to selectively remove an unwanted material or strip a thin film layer while maintaining critical dimension (CD) control and compatibility to surrounding structures, and underlying layers is crucial for a successful ULSI manufacturing technology.

When designing a wet etch process, the etch rate of each material to be etched must be known. Knowing the etch rates of other materials that will be exposed to the wet etch solution, such as masking films, surrounding structures, and underlying layers enables an etch process to be chosen for good selectivity (high ratio of etch rate of the target material to etch rate of the other material) if one exists. Etch selectivity is defined as:

$$
\text { Selectivity }=\mathrm{ER}_{\text {Target material }} / \mathrm{ER}_{\text {Other material }}
$$

While several large literature-review compilations of wet etch solutions that target

specific materials have been made ${ }^{181,182}$ they only report etch rates in some cases, and rarely have corresponding selectivity information.

The incorporation of $\mathrm{Ti}$ into the manufacturing process of advanced interconnects systems created the need for wet chemistries that address the selectivity requirements. Since wet etch chemistries that address this challenge did not exist, a selective wet etch chemical formulation capable of selectively stripping patterned $\mathrm{Ti}$ was developed throughout the course of this dissertation. The requirements for the selective wet etch chemistry are: 1) Must strip a patterned Ti thin film hard-mask layer 
with no adverse impact towards W, ILD $\left(\mathrm{SiO}_{\mathrm{x}}, \mathrm{Si}_{3} \mathrm{~N}_{4}, \mathrm{CDO}\right.$, and $\left.\mathrm{SiC}\right)$ for Via 0/Metal 1 (V0/M1) patterning, can also be referred to as first layer or first level of interconnect, 2) Must strip the thin film metal hard-mask layer with no impact to $\mathrm{Cu}$, $\operatorname{ILD}\left(\mathrm{SiO}_{\mathrm{x}}, \mathrm{Si}_{3} \mathrm{~N}_{4}, \mathrm{CDO}\right.$, and $\left.\mathrm{SiC}\right)$ for all upper interconnects layers beyond the metal 1 layer, and 3) Remove residue/polymer present on the surface as a result of the plasma etch process.

\subsection{Metals of Interest - Ti, W, and $\mathrm{Cu}$}

The properties and corrosion behaviors of titanium and tungsten are briefly discussed in this section, since much of our focus will be restricted to these two metals. Some of the basic characteristics of titanium and tungsten are listed in table $4.1^{183}$. A lengthy discussion for copper was presented in chapter 3. The knowledge acquired from the copper corrosion characterization/prevention investigation was applied in developing the $\mathrm{Ti}$ selective wet etch chemistry which exhibits compatibility to key interconnects/transistor components.

Table 4.1 Some important characteristics of titanium and tungsten

\begin{tabular}{ccc}
\hline & Ti & W \\
\hline \hline Density $\left(\mathrm{g} / \mathrm{cm}^{3}\right)$ & 4.506 & 19.25 \\
Melting point ${ }^{\circ} \mathrm{C}$ & 1668 & 3422 \\
Boiling point ${ }^{\circ} \mathrm{C}$ & 3287 & 5555 \\
Heat of fusion $\mathrm{kJ} / \mathrm{mol}$ & 14.15 & 52.31 \\
Heat of Vaporization $\mathrm{kJ} / \mathrm{mol}$ & 425 & 806.7 \\
Crystal structure & hexagonal & cubic body centered \\
Electronegativity (Pauling & 1.54 & 2.36 \\
scale) & $\left(20{ }^{\circ} \mathrm{C}\right) 0.420 \mu \Omega \cdot \mathrm{m}$ & $\left(20{ }^{\circ} \mathrm{C}\right) 52.8 \mathrm{n} \Omega \cdot \mathrm{m}$ \\
Electrical resistivity & $(300 \mathrm{~K}) 21.9$ & $(300 \mathrm{~K}) 173$ \\
Thermal conductivity & $\left(25^{\circ} \mathrm{C}\right) 8.6$ & $\left(25{ }^{\circ} \mathrm{C}\right) 4.5$ \\
W/mK & &
\end{tabular}




\subsubsection{Titanium}

Titanium is recognized for its high strength-to-weight ratio ${ }^{184}$. It is a strong metal with low density that, is quite ductile (especially in an oxygen-free environment ${ }^{185}$, lustrous, and metallic-white in color. The relatively higher melting point (over $1649^{\circ} \mathrm{C}$ ) makes it useful as a refractory metal. The most noted chemical property of titanium is its excellent resistance to corrosion; it's capable of withstanding attack by acids, moist chlorine in water but is soluble in concentrated acids ${ }^{186}$. While the Pourbaix diagram (Figure 4.4) shows that titanium is thermodynamically a very reactive metal, it is slow to react with water and air.

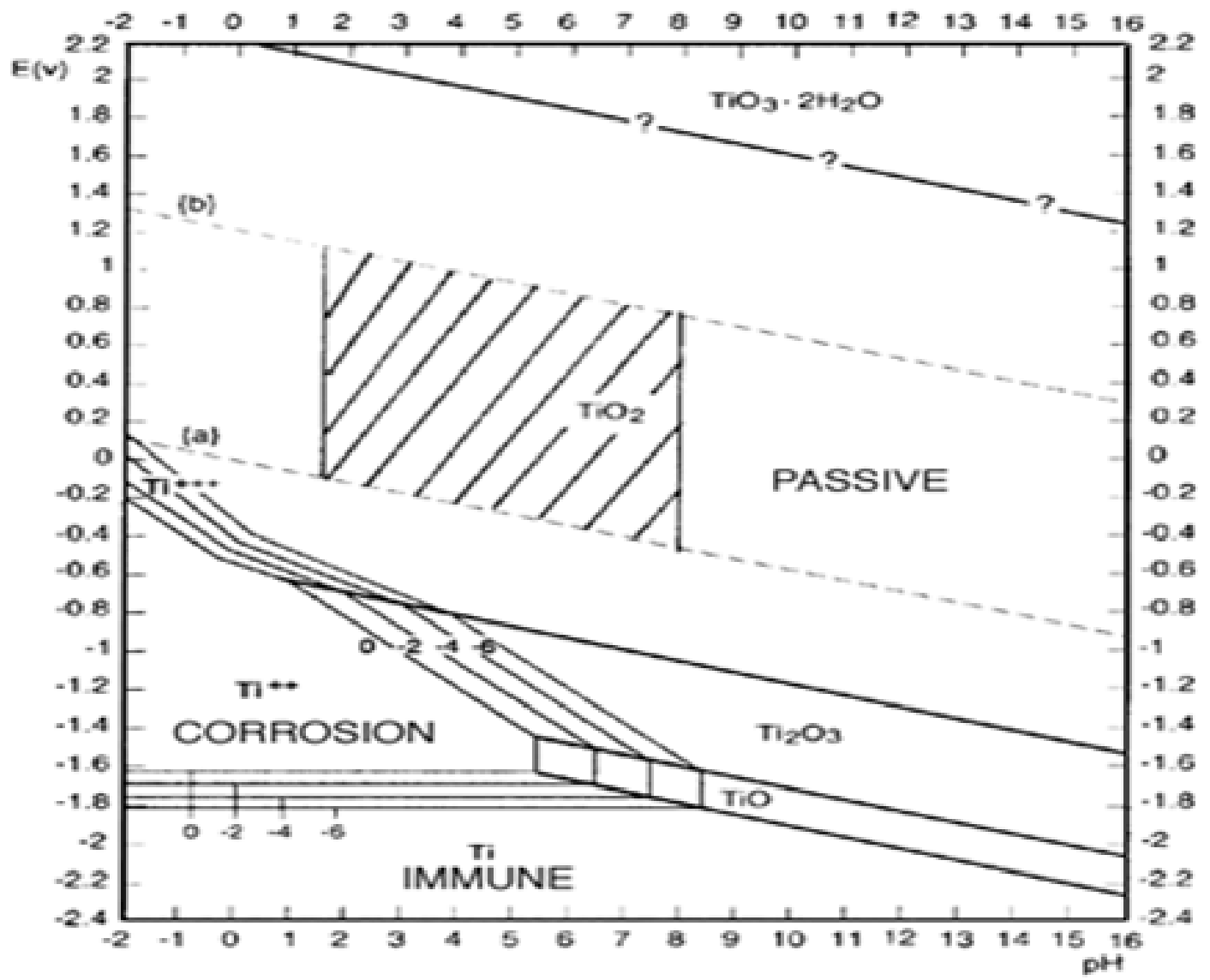

Figure 4.4 Potential-pH diagram of $\mathrm{Ti}$ in water system. 


\subsubsection{Tungsten}

In its raw form, tungsten is a steel-gray metal that is often brittle and hard to work. But, if pure, it can be worked easily ${ }^{187}$. Of all metals in pure form, tungsten has the highest melting point $\left(3422^{\circ} \mathrm{C}, 6192{ }^{\circ} \mathrm{F}\right)$, lowest vapor pressure and (at temperatures above $1650{ }^{\circ} \mathrm{C}$ ) the highest tensile strength ${ }^{188}$. Tungsten has the lowest coefficient of thermal expansion of any pure metal. The pure form is used mainly in electrical applications, but its many compounds and alloys are used in many applications. Elemental tungsten resists attack by oxygen, acids, and alkalis ${ }^{189}$. The most common formal oxidation state of tungsten is +6 , but it exhibits all oxidation states from -1 to $+6^{190}$. Tungsten typically combines with oxygen to form the yellow tungstic oxide, $\mathrm{WO}_{3}$, which dissolves in aqueous alkaline solutions to form tungstate ions, $\mathrm{WO}_{4}{ }^{2-}$. Figure 4.5 shows the Pourbaix diagram for tungsten. 


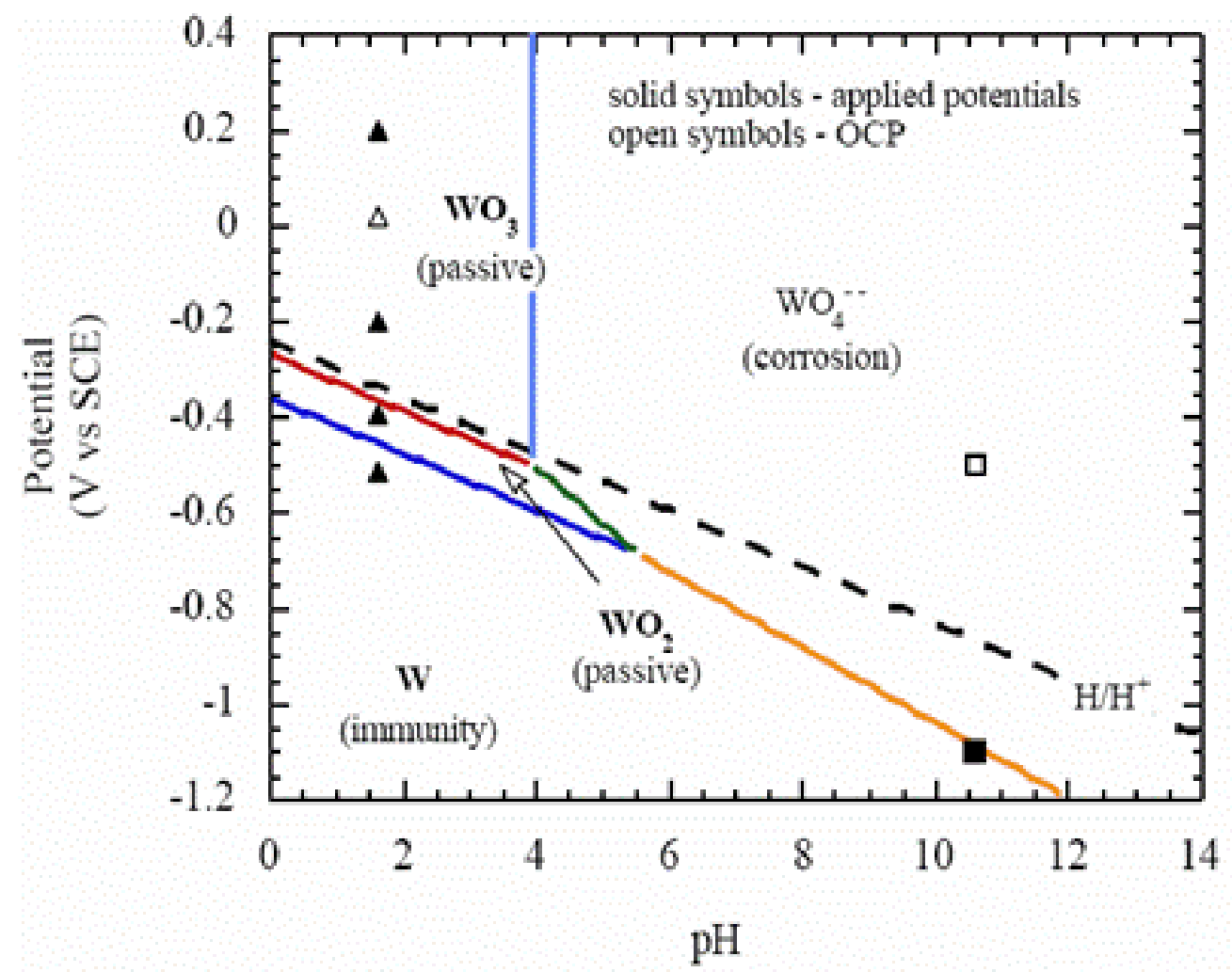

Figure 4.5 Potential-pH diagram of $\mathrm{W}$ in water system.

Pourbaix diagrams are useful tools that provide guidance or a starting point for approaching formulation development that involve metals for the application of interest. 


\section{CHAPTER 5}

\section{Selective Titanium Wet Etch/Clean Chemical \\ Formulation Development}

\subsection{Introduction}

The development of a robust titanium selective wet etch chemical formulation is herein discussed in details. The formulation is HF based chemical solution that exhibits unique selectivity behaviors and in compliance with interconnects fabrication requirements, in which a metal hard-mask is integrated for the first time to manufacture high-performance interconnects systems to enable a reliable fabrication process of advanced ULSIs. The ability to etch a titanium hard-mask without adversely impacting key interconnects structures has been successfully demonstrated. The wet etch formulation was developed to addresses the problem of selectively stripping thin, patterned Ti layer and removing post dry etch residues/polymers from a substrate with superior compatibility towards $\mathrm{W}, \mathrm{Cu}$, silicon oxide, glass silicate, carbon doped oxide, and porous ILD films.

The most distinctive characteristic of the developed formulation is that, it contains hydrofluoric acid as one of the constituents. Yet, it exhibits exceptional compatibility to silicon oxide and doped silicon oxide dielectric materials in which a zero etch rate was achieved. This unique characteristic must be emphasized, since HF containing wet etch chemistries are known to be extremely aggressive towards silicon oxides. The use of HF chemical solutions has evolved as a common practice for 
etching silicon oxides in the semiconductor industry since its inception. This is the first time, in which a hydrofluoric acid wet etch chemical solution is capable of stripping a metal while exhibiting superior compatibility to silicon oxide, doped silicon oxide, and other dielectric materials. This capability is of critical importance since it will enable a vast number of applications beyond what is presented in this dissertation.

\subsection{The Industry's Approach for Removing a Titanium Hard-Mask Film}

Although the industry was persistent on adopting a sacrificial metal hard-mask such as Ti to allow for the manufacturing of advanced high density interconnects systems, a method for robustly removing the Ti hard-mask after DD patterning steps was not available. The strategies that were exercised and attempted by the industry did not offer much success. Although the industry continued to fail in defining a path or an approach to remove $\mathrm{Ti}$ hard-mask by utilizing a variety of methods, further innovative strategies were desperately needed.

To overcome these early failures, CMP was used as a leading approach to remove the thin patterned $\mathrm{Ti}$ hard-mask film. Many issues associated with this strategy became apparent, and hindered the industry's ability to apply CMP technique for a successful manufacturing process. Discontinuity in barrier/adhesion layer, dishing in the underlying layer were key concerns resulted from the employment of a CMP process. In addition, pockets of the Ti hard-mask may still remain across the surface, which negatively impact the overall $\mathrm{K}$ value $\left(\mathrm{K}_{\mathrm{eff}}\right)$ of the ILD stack and can cause circuit shorts. The difficulty of dealing with the aforementioned challenges, a 
more fundamental solution has to be provided to robustly strip the patterned Ti hardmask layer and meet the ever stringent interconnect density requirements mandated by manufacturing high-performance microprocessors.

\subsection{Formulation Creation Strategy}

\subsubsection{Success Criteria}

Since the formulation was being developed to be implemented in the industry eventually, it needs to meet basic industry criteria. Selectivity, etch rate, economy and environmental safety and health issues (non-toxic, non-flammable and non-corrosive) are some key concerns to consider during the development process. Therefore, choice of a chemistry highly selective, with a fast but controllable etch rate, mild in nature, economical, and stable is essential to meet the success criteria devised by the semiconductor industry. In addition to the factors for selecting a suitable chemistry, device reliability and performance must not be compromised. The combination of all of these issues made this a challenging endeavor.

\subsubsection{Formulation Design Considerations}

A thorough identification of materials exposed and the underlying layer during titanium hard-mask removal is a key to a successful beginning, since the wet etch formulation must be compatible to these materials. A comprehensive investigation of all involved materials characteristics and properties is another undertaking that was performed to allow for the progression in a precise path. As illustrated by the DD structure of the first layer interconnects in Figure 4.3(c) shown in chapter 4, the titanium hard-mask layer and its relation/location with respect to other structures and 
materials are shown. Note that the formulation must be compatible to silicon oxide to account for the event of via miss alignment (unlanded via). Similarly, for upper interconnect layers beyond the first level, copper will be exposed in place of tungsten. Therefore, the formulation must also be compatible towards copper. In addition, care has to be exercised not to degrade the value of the dielectric constant which would impact the overall device reliability and performance.

\subsubsection{Development Process}

Although the Ti Pourbaix diagram (Figure 4.4) shows that titanium is thermodynamically a very reactive metal at low $\mathrm{pH}$, titanium is highly resistant to oxidizing acids over a wide range of concentrations and temperatures, only hydrofluoric acid and fluoride containing chemical solutions at a wide range of concentrations are capable of attacking titanium in an acidic environment. The use of $\mathrm{HF}$ containing solutions is problematic since it also dissolves ILD materials such as $\mathrm{SiO}_{\mathrm{x}}, \mathrm{CDO}, \mathrm{SiOF}, \mathrm{Si}_{3} \mathrm{~N}_{4}$. An alternative approach to dissolving titanium is the use of strong oxidizers such as hydrogen peroxide. The presence of a number of strong oxidizers in chemical solutions at any $\mathrm{pH}$ are capable of etching titanium. Unfortunately, the employment of oxidizers will readily dissolve tungsten and attack copper as discussed in chapter 3.

The first plan of attack in designing and developing the wet etch chemical formulation for this application was to employ a silane cross-linking agents in which a compound (monomer) from this family was added to a dilute HF solution. The preliminary hypothesis was based on the prospect that the introduction of a silicon 
based compound may hinder the HF ability to etch silicon oxide and other silicon containing dielectric materials. The low $\mathrm{pH}$ produced by HF solutions renders it compatible towards $\mathrm{W}$ as supported by the Pourbaix diagram illustrated in Figure 4.5. This inherent compatibility of HF solutions towards tungsten is a significant advantage in identifying a path to creating the formulation.

The concept of incorporating a silane cross-linking compound into a dilute HF solution appeared unrealistic at first. It has been established in the scientific community that silane cross-linking compounds and other silane molecules form solgel polymers in acidic environments ${ }^{191,192}$. Feasibility of this undertaking was demonstrated by etch rate results on blanket test wafers for the various materials involved in this work. In addition, the successful removal of the Ti layer on patterned wafers was demonstrated. Although promising results were validated and reproduced, the challenges associated with the synthesis and scalability of such a formulation in which a homogeneous (free of polymer) solution is created were enormous.

\subsection{Fundamentals of Organosilicon and Sol-Gel chemistry}

Silane cross-linking agents, silane coupling agents, and other silicon containing organic compounds belong to the organosilicon family of compounds. Organosilicon compounds are organic compounds containing carbon silicon bonds, and organosilicon chemistry is the corresponding science exploring their properties and

reactivity ${ }^{193,194}$. In general, organosilicon compounds are commonly used by many industries to synthesize polymeric materials for a variety of applications. Contrary to the industry's common practice, in this work, an organosilicon compound, 
methyltriethoxysilane (MTES) was used to create a novel chemical formulation by adding it to a dilute hydrofluoric acid solution. The concept of employing such a family of compounds to formulate a thermodynamically stable homogenous solution in an acidic environment without forming a gel/polymer is unique and contradicts with the typical scope of organosilicon compounds usage. Thus, it is necessary to discuss the chemical nature and fundamental characteristics of this family of compounds briefly and emphasize the advantages and challenges associated with their employment before continuing with detailed discussion of the formulation development process and scalability.

Sol-gel chemistry is a versatile approach for fabricating materials for numerous industrial applications. Figure 5.1 illustrates the sol-gel process in which simple molecular precursors are converted into nanometer-sized particles to form colloidal suspension, or sol. The colloidal nanoparticles are then linked with one another in a 3D, liquid-filled solid network. This transformation to a gel can be initiated by altering the reaction solution conditions. Shifting the $\mathrm{pH}$ towards acidic or basic environment is most commonly practiced to enhance gelation/polymerization. However, the acid-catalyzed reactions proceed much faster than the base-catalyzed at an equivalent catalyst concentration ${ }^{195}$. 


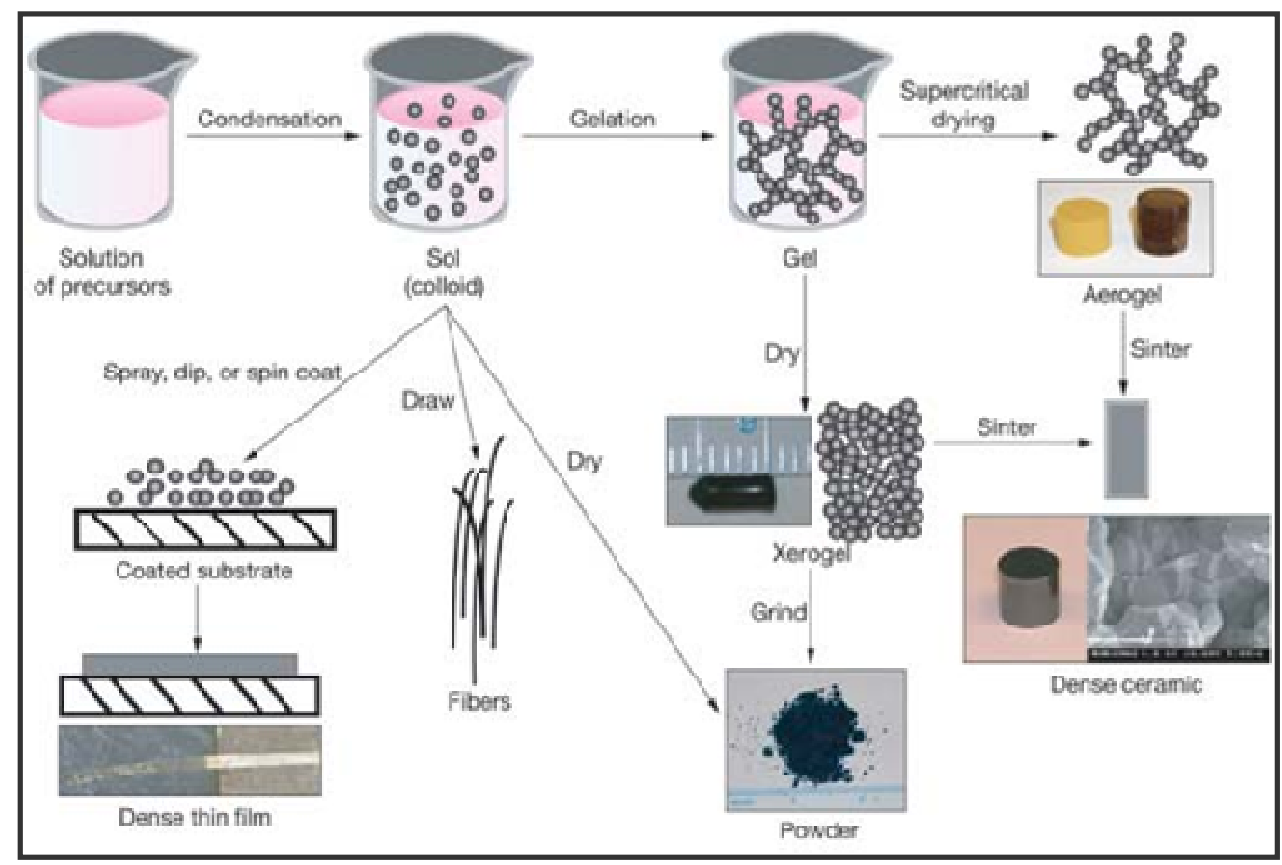

Figure 5.1 In sol-gel chemistry, molecular precursors are converted to nanometersized particles, to form a colloidal suspension, or sol. Altering the sol solution conditions produces a gel network. The sol and gel can be processed by various drying methods (shown by the arrows) to develop materials with distinct properties.

The focus here will be restricted to the methyltriethoxysilane (MTES) monomer, which was the chosen molecule from the organosilicon family. MTES is a silicon-analog of ethers or a cross-linking agent from the organotrialkoxysilane family and has $\mathrm{RSi}\left(\mathrm{OR}^{\prime}\right) 3$ as the general chemical formula. Organotrialkoxysilanes possess some notable characteristics such as instability, and in most cases, they quickly dimerize to produce siloxanes. Furthermore, difunctional variations lead to forming linear polymers. The choice of using MTES for this work was based on a number of key criterions such as availability, low cost, viscosity, and flashpoint that allow for scalability and high volume production of the formulation. 
Methyltriethoxysilane was incorporated into a dilute HF solution to achieve the desired etch rates for the various materials of interest. The synthesis process by which gel/polymer formation was prevented is discussed in the following section. Before proceeding with a detailed description of the synthesis strategy, some principle reactions for methyltriethoxysilane monomers and their relevance to this work are discussed to highlight the fundamental difficulty associated with the employment of MTES or other organosilicon compounds to ascertain a homogeneous chemical solution. The immense challenge of creating such a chemical formulation arise from the mere inherent ability of organosilicon compounds to readily form gel/polymers in an acidic environment, as a result of this basic principle, this synthesis approach has never been attempted. Moreover, to complicate matters further, gelation/polymerization is accelerated under the influence of HF. It has been reported by Bernard et al. that in a sol-gel process the hydrolysis and condensation reaction of Tetraethoxysilane (TEOS) which is a similar molecule to MTES is faster when HF is used as a catalyst in place of other acids ${ }^{196}$. The HF catalytic mechanisms to enhance the polymerization rate of a sol-gel system are discussed in a later section.

\subsubsection{The Sol-Gel Reaction}

The sol-gel reaction in past years has gained much attention in the glass and ceramic fields. This chemistry produces a variety of inorganic networks from silicon alkoxide monomer precursors. Although first discovered in the late 1800s and extensively studied since the early 1930 s, a renewed interest ${ }^{197,198}$ surfaced in the early 1970s when monolithic inorganic gels were formed at low temperatures and converted 
to glasses without a high temperature melting process ${ }^{199}$. Through this process, homogeneous inorganic oxide materials with desirable properties of hardness, optical transparency, chemical durability, tailored porosity, and thermal resistance, can be produced at room temperatures, as opposed to the much higher melting temperatures required in the production of conventional inorganic glasses ${ }^{200,201}$. The specific uses of these sol-gel produced glasses and ceramics are derived from the various material shapes generated in the gel state, i.e., monoliths, films, fibers, and monosized powders. Sol-gel chemistry is used in many applications, including, optics, protective and porous films, optical coatings, window insulators, dielectric and electronic coatings, high temperature superconductors, reinforcement fibers, fillers, and catalysts ${ }^{149}$.

At the functional group level, three reactions are generally used to describe the sol-gel process: hydrolysis, alcohol condensation, and water condensation. In general, the hydrolysis reaction represented by equation 5-1 shows that the addition of water replaces alkoxide groups (-OR) with hydroxyl groups $(\mathrm{OH})$.

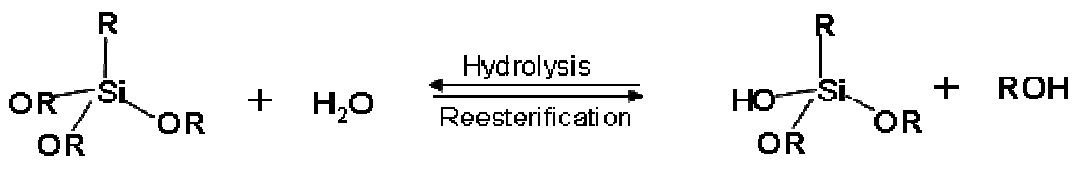

Subsequent condensation reactions (Equations 5-2 and 5-3) involving the silanol groups $(\mathrm{Si}-\mathrm{OH})$ produce siloxane bonds $(\mathrm{Si}-\mathrm{O}-\mathrm{Si})$ plus the by-products water or alcohol. Under most conditions, condensation commences before hydrolysis is 
complete. However, conditions such as, $\mathrm{pH}$, molar ratio of the various species, and catalyst can force completion of hydrolysis before condensation begins ${ }^{149}$.
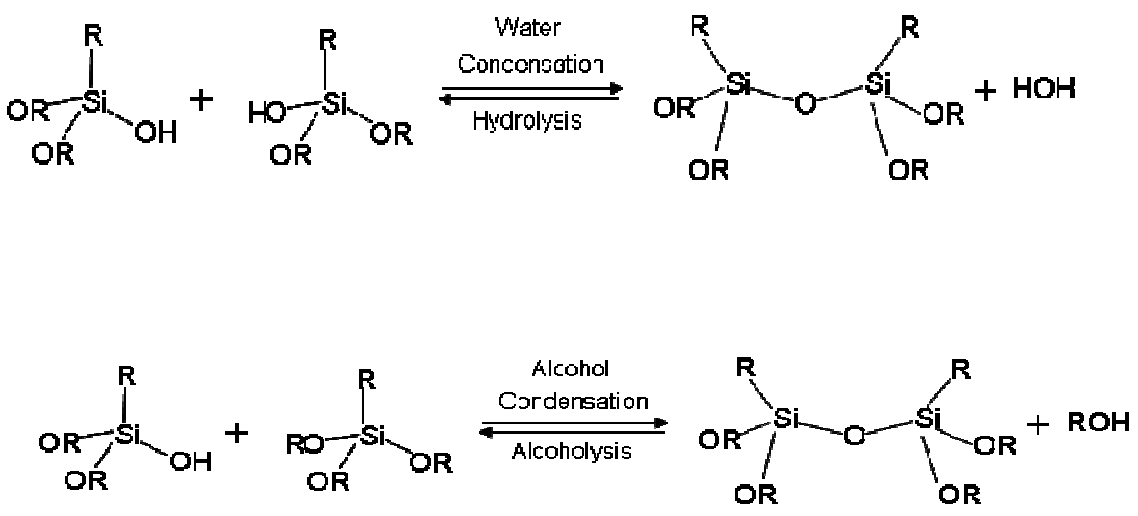

Under acidic conditions, it is likely that an alkoxide group is protonated in a rapid first step. Electron density is withdrawn from the silicon atom, making it more electrophilic and thus more susceptible to attack from water. This results in the formation of a penta-coordinate transition state with significant SN2-type character ${ }^{202}$. The transition state decays by displacement of an alcohol and inversion of the silicon tetrahedron, as seen in Figure 5.2.

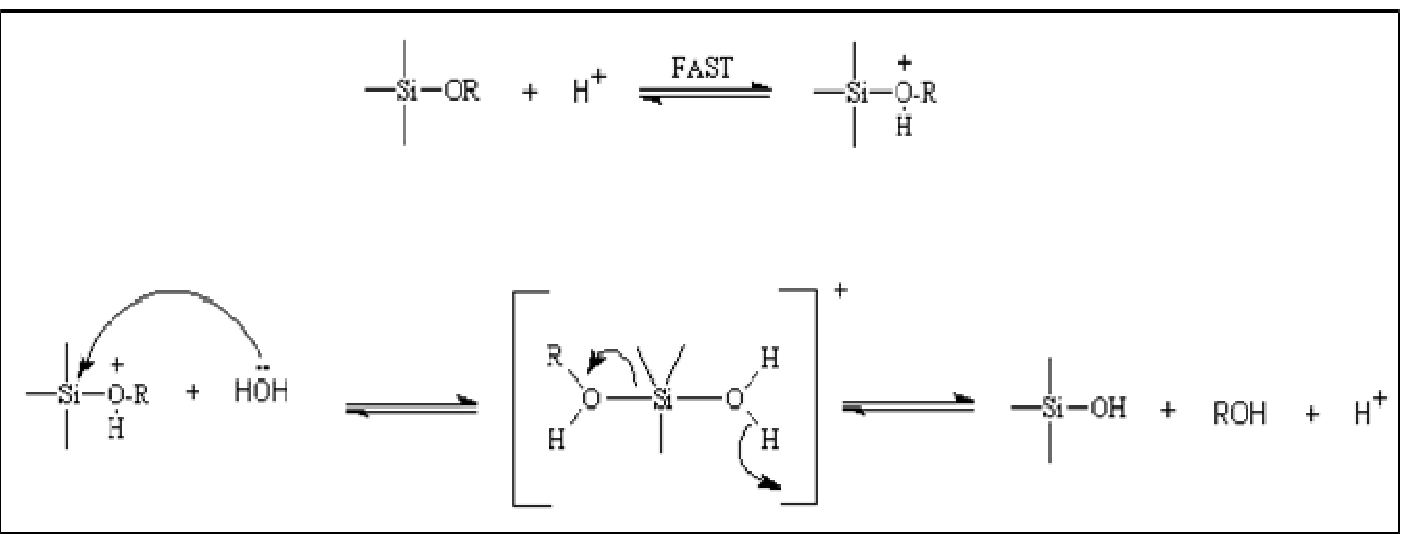

Figure 5.2 Acid-catalyzed hydrolysis of Silicon Alkoxide. 
With respect to the hydrolysis and condensation reactions of the sol-gel process, It has been established that certain reaction parameters are more important than others ${ }^{203,204,205,206,207208}$. The $\mathrm{pH}$ has a profound influence on hydrolysis and polymerization. Although other factors such as the nature and concentration of catalyst, and $\mathrm{H}_{2} \mathrm{O} / \mathrm{Si}$ molar ratio have significant effect on the sol-gel process, the $\mathrm{pH}$ emerges as one of the most influential factors. The effect of $\mathrm{pH}$ on the hydrolysis represented by reaction (5-1) is illustrated in Figure 5.3. The hydrolysis rate dependency on the $\mathrm{pH}$ value $\mathrm{e}^{209}$ is clearly shown. Furthermore, $\mathrm{F}^{-}$concentration and $\mathrm{pH}$ influence on condensation/polymerization time is illustrated in Figure 5.4 $4^{210,211}$.

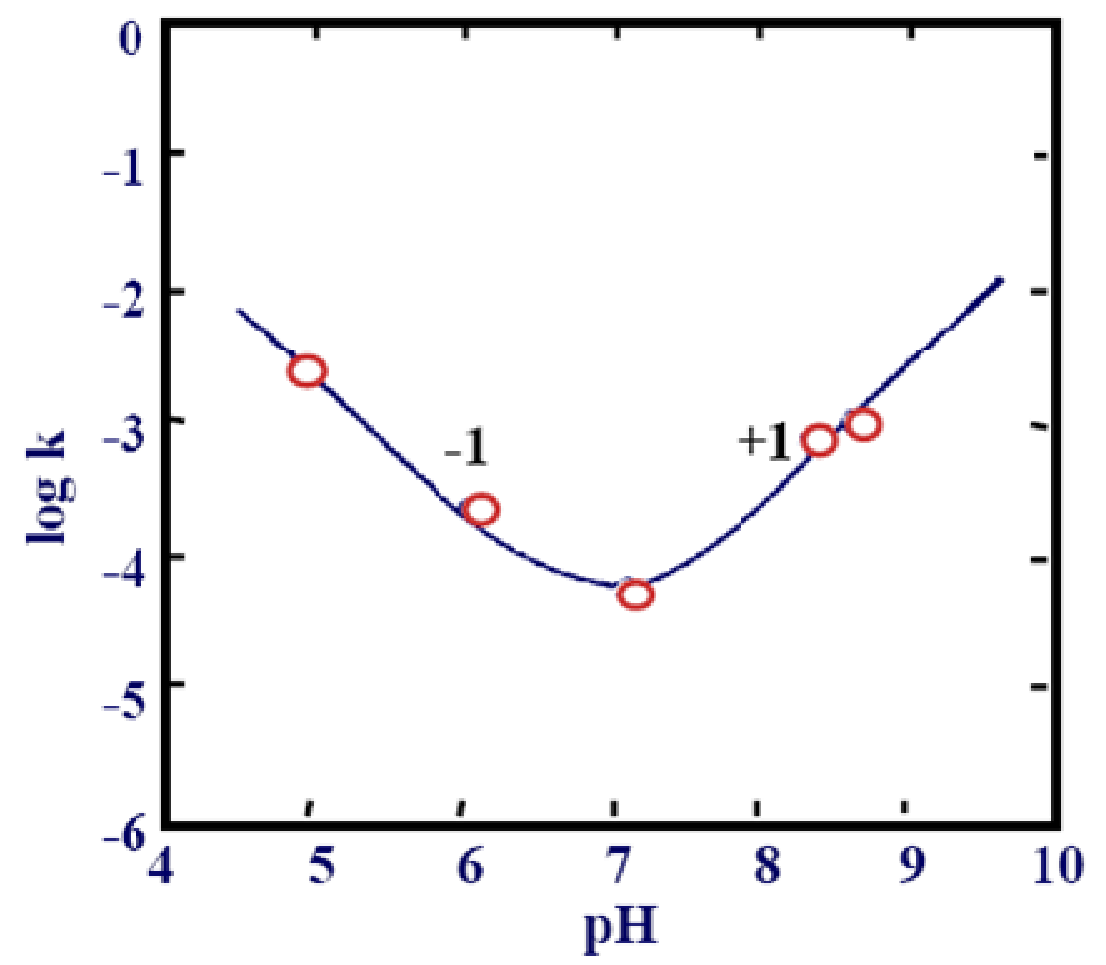

Figure 5.3 Hydrolysis rate as a function of $\mathrm{pH}$ for $\mathrm{RSi}(\mathrm{OR}) 3+\mathrm{H} 2 \mathrm{O}=\mathrm{RSi}(\mathrm{OR}) 2 \mathrm{OH}$ $+\mathrm{ROH}$. 


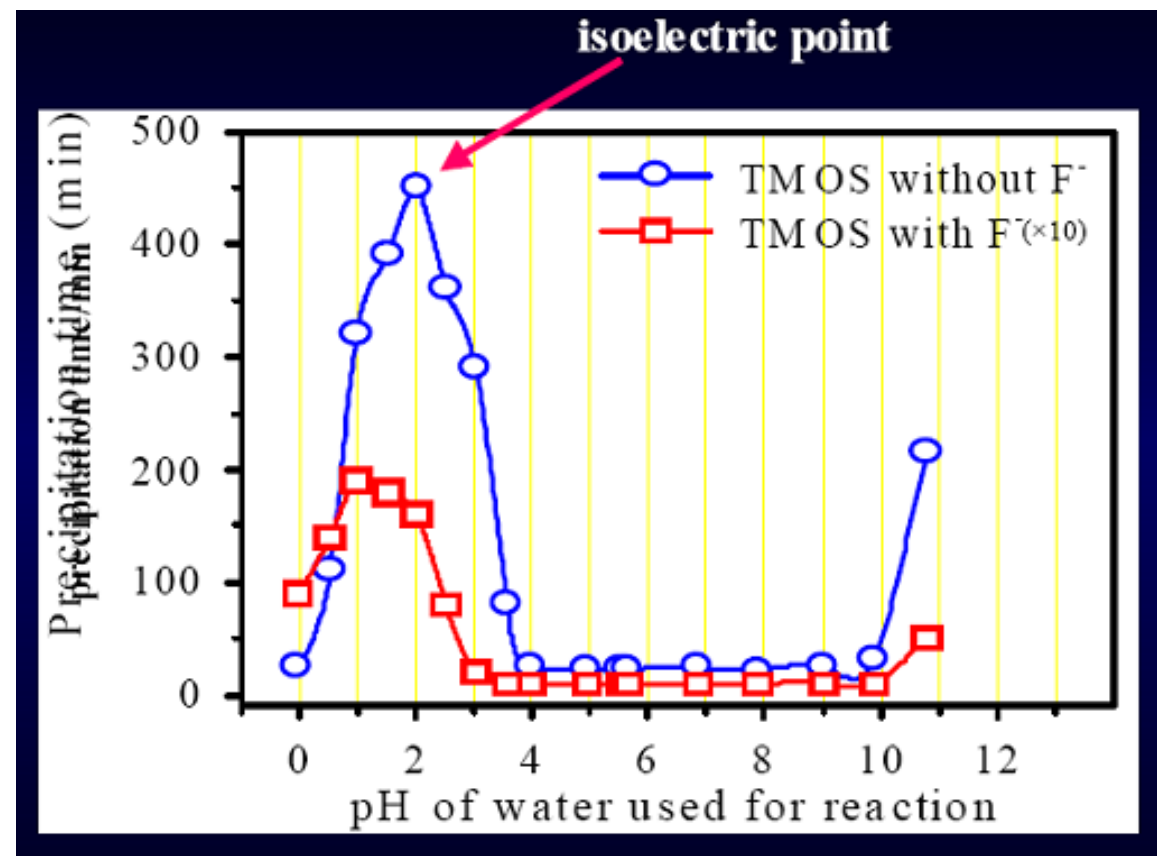

Figure 5.4 Polymerization Time dependence on $\mathrm{pH}$ and $\mathrm{F}^{-}$concentration.

\subsection{Experimental Design}

In summary, the developed wet etch/clean formulation must be synthesized in such a way that, a homogenous solution mixture free of particles, polymer, or oily residue is created. Techniques including screening and factorial experiments were undertaken to define a robust process window and process cliffs with respect to the formulation synthesis and etch rates of thin films materials.

The development process was segmented into a number of phases which included basic science evaluation of literature, components Research, scalability, and cleanability (the ability to remove post plasma etch residue) evaluations. Key responses were divided into two main categories. The first is related to the formulation synthesis process, while the second is associated with creating a formulation that meets the etch rate requirements. The synthesized formulation was evaluated based on the final solution mixture characteristics. In regard to etch rates, 
the formulation must be capable of robustly stripping Ti while maintaining a zero etch rate towards $\mathrm{W}, \mathrm{SiO}_{\mathrm{x}}, \mathrm{CDO}, \mathrm{Si}_{3} \mathrm{~N}_{4}, \mathrm{SiC}$, and $\mathrm{Cu}$ throughout the wet etch process.

\subsection{Strategy}

To achieve the goal a methodical approach was defined and followed. The following are the steps that were undertaken: 1) the chemical formulation constituents and concentrations to carry out the synthesis successfully were identified, 2) it was made certain that the synthesis process, byproducts, and final formulation mixture are safe, 3) the synthesis approach and key parameters such as temperature, time, etc... were identified, 4) the process target, process window, and process cliffs with respect to the synthesis and etch rates of the investigated materials were determined, and 5) refinement and optimization of the synthesis to produce a suitable and robust formulation that meets the required etch rates targets was performed.

\subsubsection{Identifying the Formulation Constituents}

Previous discussion has emphasized the detrimental affects of using oxidizers such as $\mathrm{H}_{2} \mathrm{O}_{2}$ and/or HF to strip titanium. The predicament with using oxidizers is that they would negatively impact the tungsten, while HF would have severe consequences towards $\mathrm{SiO}_{\mathrm{x}}$ and ILD materials. The incorporation of MTES into a dilute HF solution to synthesize a formulation that suppresses the impact of $\mathrm{HF}$ on $\mathrm{SiO}_{\mathrm{x}}$ emerged as a feasible composition. Screening factorial experiments of the identified components (HF/MTES/Water) were carried out.

From a wet etch selectivity point of view, the proposed composition of HF, water, and MTES is compatible only at the first interconnect level in which tungsten is 
exposed during Ti removal as illustrated and discussed in previous sections. In order to allow the employment of the formulation for all other interconnect layers, in which copper is exposed during the Ti strep process, it must exhibit compatible behaviors towards $\mathrm{Cu}$. To enable this formulation which would not corrode copper, the use of an organic copper corrosion inhibitor emerged as a practical approach. Benzotriazole (BTA) was selected as the corrosion inhibitor for this application. The selection and characterization work is discussed later.

\subsubsection{Safety Considerations}

In addition to performance criterion previously summarized with respect to synthesis and etch rates, the formulation must be safety compliant and exhibit minimal impact on the environment and human health. In the early stages of this work, it was anticipated that $\mathrm{SiF}_{4}$ can potentially form during the synthesis and could pose serious health concern. $\mathrm{SiF}_{4}$ is known to be a toxic gas and would limit the ability to scale the

production of the formulation for high volume manufacturing. To address this possibility, a study of head-space chemical analysis during the synthesis was undertaken. Examples of reactions leading to formation of $\mathrm{SiF}_{4}$ are given by:

$$
\begin{aligned}
& \mathrm{CH}_{3} \mathrm{Si}\left(\mathrm{OCH}_{2} \mathrm{CH}_{3}\right)_{3}+\mathrm{HF} \rightarrow \mathrm{CH}_{3} \mathrm{Si}(\mathrm{OH})_{3}+{ }_{3} \mathrm{CH}_{3} \mathrm{CH}_{2} \mathrm{OH} \uparrow \\
& \mathrm{CH}_{3} \mathrm{Si}(\mathrm{OH})_{3}+\mathrm{HF} \rightarrow \mathrm{SiF}_{4} \uparrow+\ldots
\end{aligned}
$$

Lab scale head-space extractive Fourier Transform Infrared Spectroscopy (FTIR) analysis was carried out to monitor $\mathrm{SiF}_{4}$ concentration as MTES was dispensed into the dilute HF solution. 


\subsubsection{Experimental Details}

An FTIR Unicam-Mattson spectrometer was used for this analysis, at $0.5 \mathrm{~cm}^{-1}$ resolution, and 150C.A $10 \mathrm{~cm}$ single pass gas cell was used. The sample line was positioned directly above the solution in the hood. A sample pump was used to extract the sample through the FTIR cell. The $\mathrm{SiF}_{4}$ detection limit was estimated to be $6 \mathrm{ppm}$ using this procedure (excluding the effects of interferences which could raise the detection limit). Several experiments in which the Silicon precursor was added to reaction solution were tested.

\subsubsection{Results}

Figure 5.5 shows background spectrum before MTES addition to the dilute HF solution. Figure 5.6 shows the $\mathrm{SiF}_{4}$ calibration spectrum $(10 \mathrm{ppm})$. The FTIR spectrum after addition of silicon precursor MTES is illustrated in Figure 5.7. Figure 5.8 spectra shows $\mathrm{SiF}_{4}$ calibration superimposed on silicon precursor addition spectra.

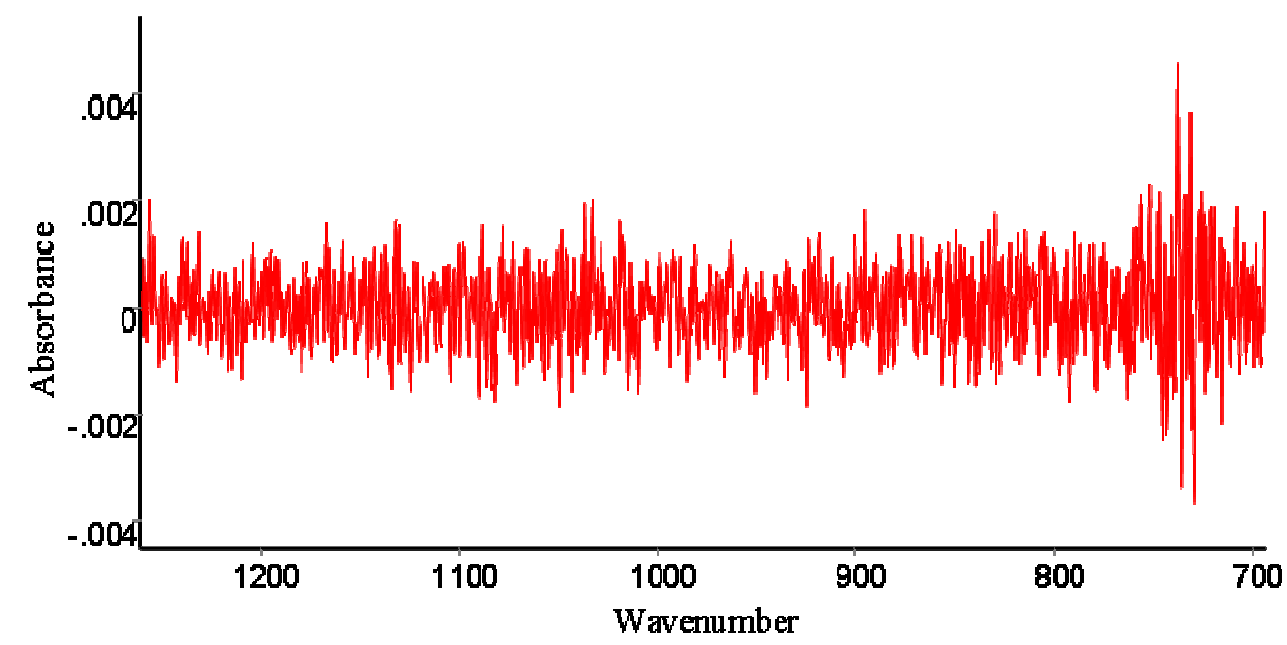

Figure 5.5 Background spectrum - prior to precursor (MTES) addition. 


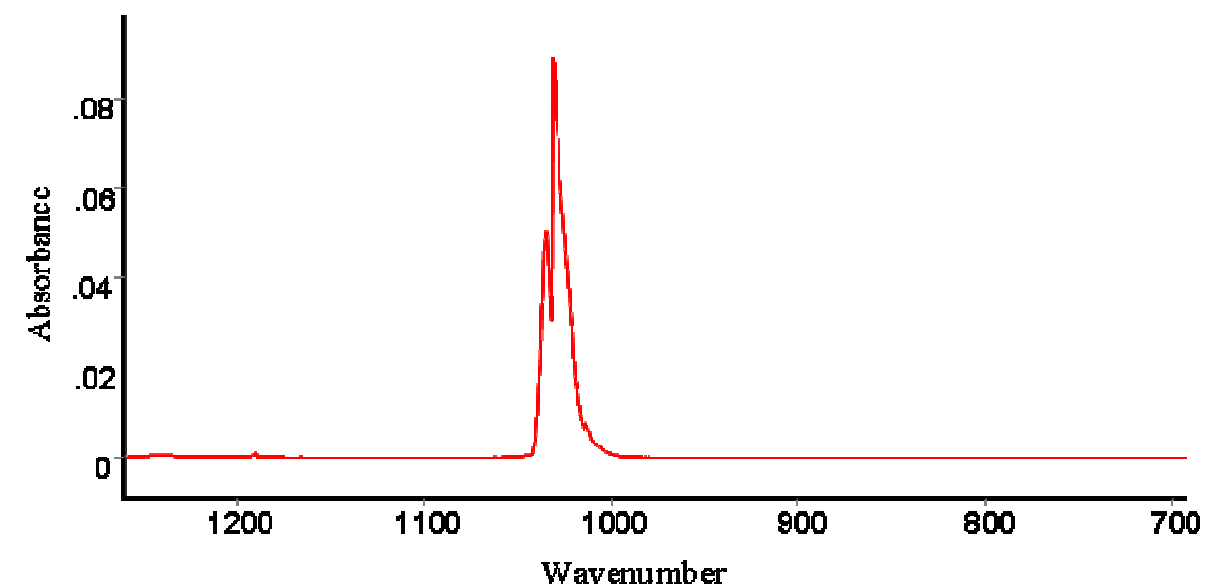

Figure 5.6 $\mathrm{SiF}_{4}$ calibration spectrum $(10 \mathrm{ppm})$.

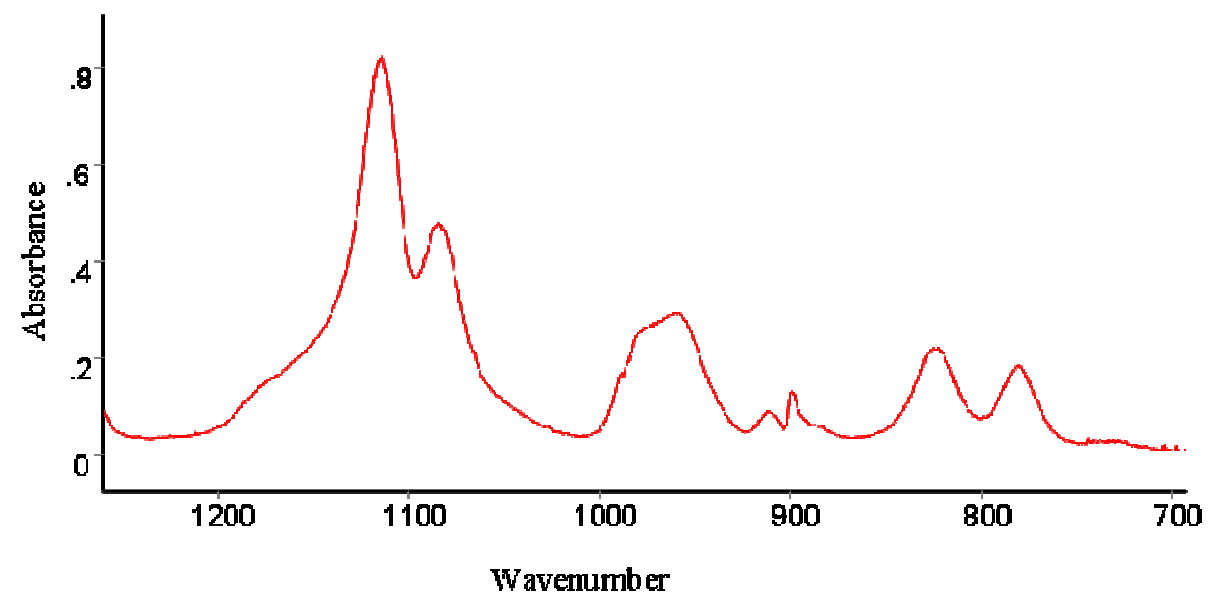

Figure 5.7 FTIR spectrum after addition of silicon precursor MTES.

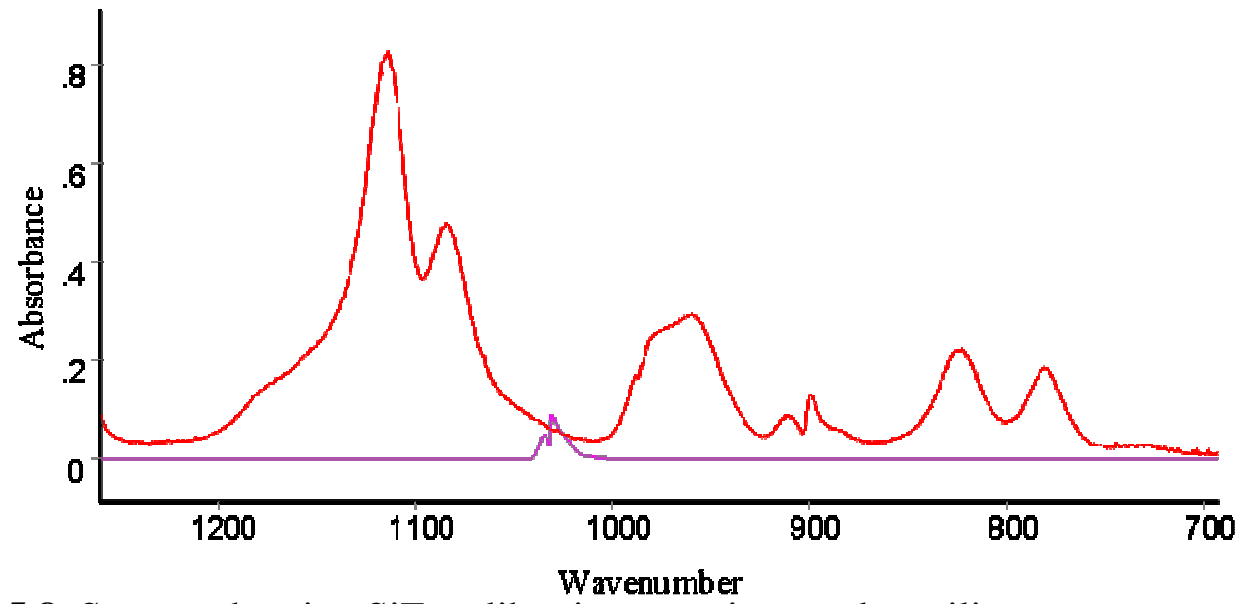

Figure 5.8 Spectra showing $\mathrm{SiF}_{4}$ calibration superimposed on silicon precursor addition. 
Small emissions of $\mathrm{SiF}_{4}$ may have been detected; however VOC emissions from formulation were the predominant component in headspace above reaction. Therefore, the formulation is considered safe.

\subsubsection{Formulation Synthesis Process}

The aim of this section is to underscore key parameters that were investigated in carrying out the synthesis. It was readily established that factors such as temperature, concentrations, reaction time, Stir rate, and MTES addition rate to the dilute HF solution are highly influential in synthesis of formulation with desired characteristics.

Screening and factorial experiments were undertaken to understand the relationship between the aforementioned synthesis parameters/conditions, concentrations of mixture constituents, and the formation of particles, residue, or polymer, i.e., sol-gel. Furthermore, once a process window with respect to synthesis was determined, additional factorial Design of Experiments (DOE) were conducted within this process window space to identify exact concentrations of constituents to produce a final formulation mixture that meets the etch rate requirements for the investigated substrates. In addition, an evaluation of the formulation's capability to strip the Ti layer on DD patterned wafers in particular, was conducted.

Since the formulation deals with a three-component system, HF, MTES, and water, the synthesis process window and process cliffs were identified through the employment of a triangular phase diagram as a practical approach to investigate the interdependency of the various factors and conditions to achieve a formulation with 
the desired characteristics. A three component system has two degrees of freedom $F=$ 2. However, when other factors are involved the degrees of freedom, and hence, variance may significantly increase making the task of examining the interdependency of the various factors and variations in compositions of the mixture extremely complex. Therefore, it was important to first, determine the optimal reaction conditions/parameters, and then hold them constant once they are identified. This strategy resulted in reducing the degrees of freedom to two; which allows for the focus to be limited to the concentrations of the three components to achieve the desired etch rates.

The phase diagram was constructed based on the volume fractions of the three component system which is satisfied by

$$
\mathrm{X}_{\mathrm{HF}}+\mathrm{X}_{\mathrm{MTES}}+\mathrm{X}_{\mathrm{H} 2 \mathrm{O}}=1
$$

The aforementioned investigation is illustrated in Figure 5.9 in which the Ti selective wet etch/clean formulation synthesis process cliffs and process window are identified. 


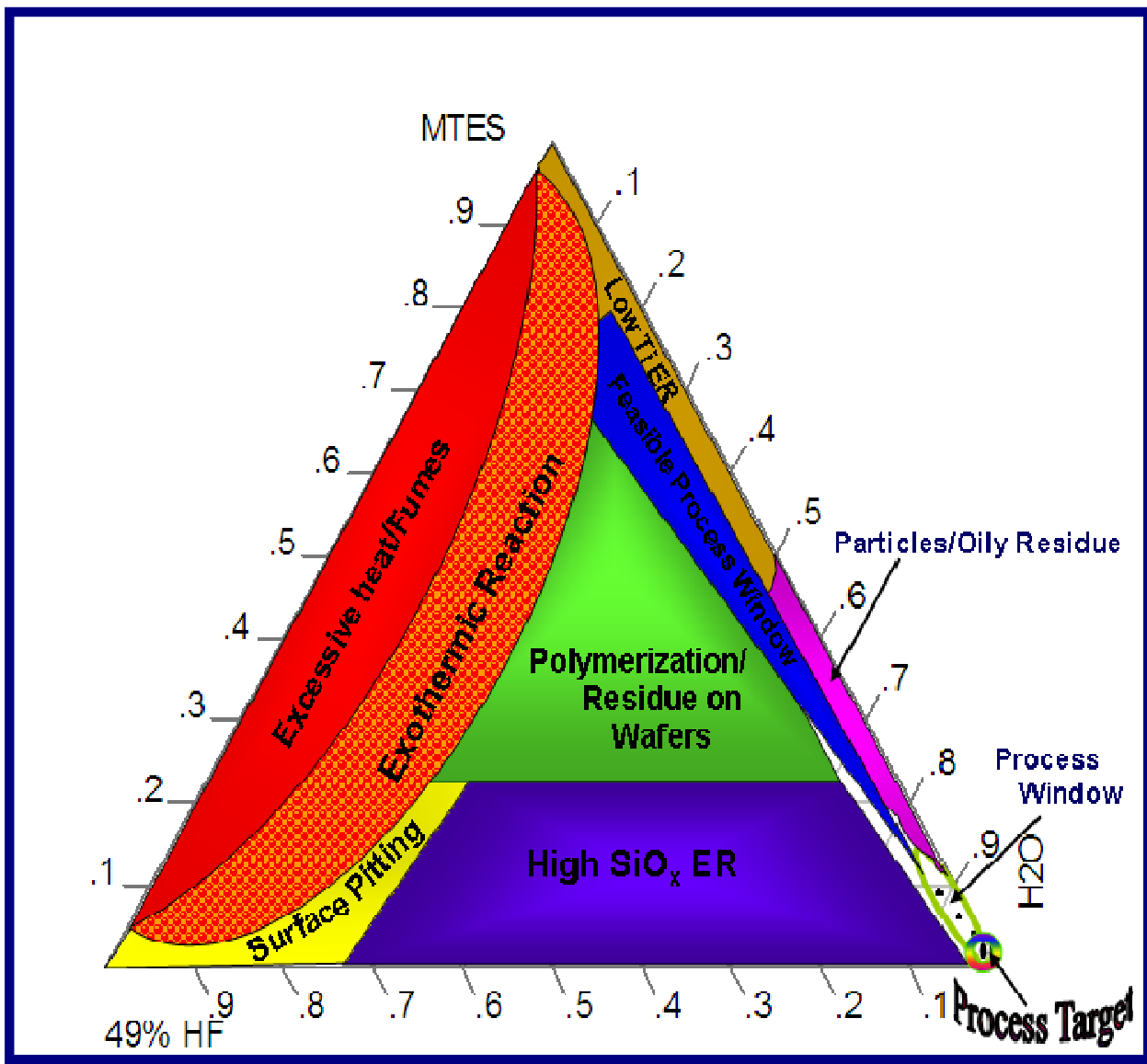

Figure 5.9 Process cliffs affecting Ti selective wet etch/clean formulation.

Silicon oxide etch rate suppression was demonstrated by the incorporation of MTES into the dilute HF solution when the proper conditions/parameters are applied. From an etch rate selectivity prospective, the next logical step is to extensively explore the synthesis operating process window to pin point the location (process target) of the final formulation composition that provides the desired etch rates for the investigated thin film materials and capable of selectively stripping the Ti layer on DD patterned wafers. A factorial DOE approach was carried out to achieve such a task in which a formulation with robust Ti ER and zero or near zero ER for all other materials of interest is realized. 


\subsection{Synthesis Details and Reaction Characterization}

The challenges of establishing the formulation became more intense with each level of scalability as progress was made to move from a smaller container to a larger one. Before scaling the synthesis to larger containers and reactors, Initial work was done on a laboratory scale, at a beaker level. The reaction vessel evolved from a 200 $\mathrm{ml}$ beaker, to a 3000 gallon reactor. The problems associated with the chemical formulation scalability will be discussed in chapter 7. Synthesis detailed work on a small scale (beaker level) is described in the following sections.

\subsubsection{Mixing, and Reaction Optimization}

The dilute HF solution is prepared using a concentrated 49\% HF. This solution is then heated to $80^{\circ} \mathrm{C}$. Pure MTES is added to the dilute HF solution at 80 ${ }^{\circ} \mathrm{C}$. The volumes used to carry out the synthesis are $200.0 \mathrm{ml}$ water, $1.0 \mathrm{ml} \mathrm{HF}$, and $3.80 \mathrm{ml}$ MTES. The MTES is added to the solution at a rate of $0.5 \mathrm{ml} /$ minute using a digital pipette while vigorously stirring the solution mixture using a magnetic stir bar.

Throughout the synthesis, the temperature is maintained at $80{ }^{\circ} \mathrm{C}$ and vigorous mixing is applied. For the reaction to reach completion a period of at least two hours is required. The solution mixture is then filtered and allowed to cool to room temperature. At this point blanket test wafers and patterned wafers are processed in the solution mixture. The etch rate functional testing for blanket wafers with various substrates and evaluation of Ti assisted DD patterned wafers were performed at $60^{\circ} \mathrm{C}$. 


\subsubsection{Proposed Reaction}

Although the synthesis of the formulation deals with a complex reaction, some of the reaction products were determined and characterized as shown in chapter 6 . Due to the large number of possible product species, many still remain not fully identified. An extensive analytical work was undertaken in an attempt to identify as many of the product as can be possible.

Figure 5.10 depicts the proposed synthesis reaction and shows the two possible pathways. One reaction pathway is leads to the formation of polymer, while the other leads to the creation of homogenous solution comprised of the various product species. In order to achieve the latter and ensure the absence of polymer, factors and conditions discussed in the previous section must be taken into account and precisely controlled. From a kinetics point of view this can be accomplished if $\mathrm{K}_{2}$ is $>\mathrm{K}_{1}$. It was found that this condition can be satisfied by the following criterion: 1) The optimal temperature range is $70-80{ }^{\circ} \mathrm{C}$ throughout the synthesis. 2) The desired concentration ratio of HF to MTES was identified and maintained constant as long as the water volume is in excess. 3) MTES must be added at a slow rate into the dilute HF solution at $70-80{ }^{\circ} \mathrm{C}$. The optimal rate of addition was found to be $0.5 \mathrm{ml} / \mathrm{minute}$ or below for a $200 \mathrm{ml}$ dilute HF solution in a beaker. 4) While adding MTES and during the synthesis aggressive mixing must be sustained. 


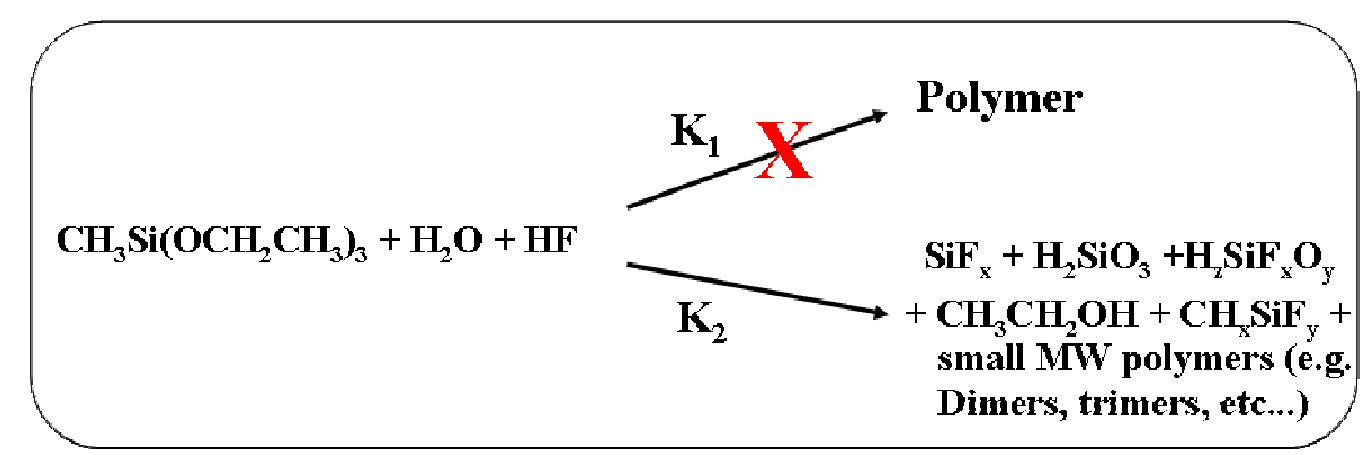

Figure 5.10 Proposed reaction for the Ti selective etch formulation synthesis.

\subsection{Etch Rate Experiments}

The silicon oxide, silicon nitride, carbon doped silicon oxide, silicon carbide, and metals, $\mathrm{Cu}, \mathrm{W}$, and Ti were deposited using well established deposition techniques. Etch rate investigation for the various thin film substrates were performed by measuring the thickness before and after wafer immersion in the developed formulation solution. The thickness measurements for $\mathrm{SiOx}, \mathrm{CDO}, \mathrm{Si}_{3} \mathrm{~N}_{4}, \mathrm{SiC}$ were performed using an ellipsometer (Nanometrix, nanospec 5100). The titanium, tungsten, and copper film thickness measurements were performed utilizing a four point probe method (Surface resistivity meter, Gaurdian Manufacturing Inc., Model SRM-232). All experiments were carried out at $60^{\circ} \mathrm{C}$, using high density poly propylene (HDPP) beaker.

\subsubsection{Etch Rate Targeting and Optimization}

A factorial DOE was carried out to determine the exact concentrations for the formulation constituents in which desired etch rates can be realized and a process target can be achieved. Initial screening experiments within the synthesis operating window depicted in Figure 5.9 showed that W, CDO, and SiC are not affected by 
variations in concentrations within this space. $\mathrm{Ti}, \mathrm{SiO}_{\mathrm{x}}$, and $\mathrm{Si}_{3} \mathrm{~N}_{4}$ etch rates did vary as a function of concentrations and hence were extensively investigated.

The following are the involved factors and response:

Factors: HF, MTES, and water concentrations by volume.

Response: $\mathrm{Ti}, \mathrm{SiO}_{\mathrm{x}}$, and $\mathrm{Si}_{3} \mathrm{~N}_{4}$ etch rates.

The factorial was designed to achieve the final formulation solution mixture that provides an optimal process with respect to etch rates, and suitable for Ti assisted DD patterning applications.

The etch rate investigation results as a function of volumes are illustrated in table 5.1. All experiments were performed at $60{ }^{\circ} \mathrm{C}$. To demonstrate synthesis and etch rate control and reproducibility, synthesis was carried out in duplicates and etch rate experiments were performed in triplicates. A temperature of $60{ }^{\circ} \mathrm{C}$ was chosen due to its beneficial enhancement from a cleanability, and etch rate robustness perspectives. Etch rate is directly proportional to temperature. In addition, $60{ }^{\circ} \mathrm{C}$ is a practical temperature that can be implemented for high volume manufacturing. 
Table 5.1 Etch rates as a function of formulation constituents concentrations

\begin{tabular}{|c|c|c|c|c|c|c|c|c|}
\hline $\begin{array}{c}49 \% \text { HF } \\
\text { Volume } \\
(\mathrm{ml})\end{array}$ & $\begin{array}{c}\text { MTES } \\
\text { Volume } \\
(\mathrm{ml})\end{array}$ & $\begin{array}{c}\mathrm{H} 2 \mathrm{~V} \\
\text { Volume } \\
(\mathrm{ml})\end{array}$ & $\begin{array}{c}\text { Ti ER } \\
(\mathrm{A} / \mathrm{min}) \\
60 \mathrm{C}\end{array}$ & $\begin{array}{c}\text { CVD SiOx } \\
\text { ER } \\
(\mathrm{A} / \mathrm{min}) \\
60 \mathrm{C}\end{array}$ & $\begin{array}{c}\text { Si3N4 ER } \\
(\mathrm{A} / \mathrm{min})\end{array}$ & $\begin{array}{c}\text { W ER } \\
(\mathrm{A} / \mathrm{min})\end{array}$ & $\begin{array}{c}\text { CDO ER } \\
(\mathrm{A} / \mathrm{min})\end{array}$ & $\begin{array}{c}\text { SiC ER } \\
(\mathrm{A} / \mathrm{min})\end{array}$ \\
\hline \hline 1 & 6 & 200 & 24.000 & -1 & 0.000 & 0 & 0 & 0 \\
\hline 1 & 6 & 200 & 24.000 & -1.1 & 0.000 & 0 & 0 & 0 \\
\hline 1 & 5 & 200 & 43.103 & -0.6 & 0.000 & 0 & 0 & 0 \\
\hline 1 & 4.5 & 200 & 56.604 & -0.1 & 0.000 & 0 & 0 & 0 \\
\hline 1 & 4.5 & 200 & 56.604 & -0.2 & 0.000 & 0 & 0 & 0 \\
\hline 1 & 3.8 & 200 & 120.000 & 0 & 0.000 & 0 & 0 & 0 \\
\hline 1 & 3.8 & 200 & 120.000 & 0 & 0.000 & 0 & 0 & 0 \\
\hline 1 & 3.8 & 200 & 120.000 & 0 & 0.000 & 0 & 0 & 0 \\
\hline 1 & 3.6 & 200 & 148.515 & 0.4 & 0.014 & 0 & 0 & 0 \\
\hline 1 & 3.3 & 200 & 254.237 & 2.6 & 0.090 & 0 & 0 & 0 \\
\hline 1 & 3.3 & 200 & 254.237 & 2.6 & 0.090 & 0 & 0 & 0 \\
\hline 1 & 3 & 200 & 288.462 & 3.7 & 0.128 & 0 & 0 & 0 \\
\hline 1 & 3 & 200 & 288.462 & 3.8 & 0.131 & 0 & 0 & 0 \\
\hline 1 & 2.8 & 200 & 306.122 & 4.5 & 0.155 & 0 & 0 & 0 \\
\hline 1 & 2.8 & 200 & 306.122 & 4.5 & 0.155 & 0 & 0 & 0 \\
\hline 1 & 2.4 & 200 & 357.143 & 6.8 & 0.234 & 0 & 0 & 0 \\
\hline 1 & 2.4 & 200 & 357.143 & 6.8 & 0.234 & 0 & 0 & 0 \\
\hline 1 & 0 & 200 & 1000.000 & 306 & 10.552 & 0 & 0 & 0 \\
\hline 0 & 6 & 200 & 0.000 & 0 & 0.000 & 0 & 0 & 0 \\
\hline 0 & 3 & 200 & 0.000 & 0 & 0.000 & 0 & 0 & 0 \\
\hline 0 & 0 & 200 & 0.000 & 0 & 0.000 & 0 & 0 & 0 \\
\hline & & & & & & & & \\
\hline
\end{tabular}

\subsection{DOE analysis and Response Surface Modeling}

The DOE analysis and response surface modeling were performed utilizing JMP (John Manhattan Project) software (SAS associates). The data in table 5.1 was used to fit a model and carry out the analysis. Furthermore, the fitted model was employed to derive the response surface etch rate contour plots. The strategy that was used to carry out this statistical analysis is as follows: 1) factorial screening studies were performed, 2) additional experimentation to verify and support screening results were executed, 3) second-order design and modeling to characterize the promising region (process window) and seek optimum factor settings (compositional concentrations) were conducted, and 4) validation/verification experiments to confirm and fine tune predicted optimal factors parameters (concentrations) were carried out. Factorial DOEs are very useful tools in identifying the most influential factors (factor screening) and for understanding how these factors affect the response variables 
within the experimental region. In this work, once the influential factors, HF and MTES and their interactions were identified, a Response Surface Methodology (RSM) was used for optimizing the response (etch rates) and locating the process window and process target. RSM is a collection of mathematical and statistical techniques that are useful tools for modeling and analysis.

\subsection{DOE Results}

The fitted model analysis outcome for each response, $\mathrm{Ti}, \mathrm{SiO}_{\mathrm{x}}$, and $\mathrm{Si}_{3} \mathrm{~N}_{4}$ etch rates are presented in appendix $\mathrm{B}$. This analysis provides the etch rate prediction expressions, shown in figures $5.11,5.13$, and 5.15 for $\mathrm{Ti}, \mathrm{SiO}_{\mathrm{x}}$, and $\mathrm{Si}_{3} \mathrm{~N}_{4}$ respectively. Although the computer generated expressions show too many significant figures, the actual is only three significant figures. Another artifact of the JMP program expressions is that they include unnecessary terms (terms multiplied by zero). Since the DOE deals with 3 factors, the regression model is a selected subset of terms from a third-order Taylor series approximation. The interaction profiles for $\mathrm{Ti}, \mathrm{SiO}_{\mathrm{x}}$, and $\mathrm{Si}_{3} \mathrm{~N}_{4}$ etch rates are shown in Figures 5.12, 5.14, and 5.16 respectively, in which a matrix of interaction plots are shown in each figure. This is a useful tool in which two-factor interaction effects can be visualized. Note that non parallel lines are an evidence of interaction. It is readily observed that MTES and HF interaction effect is significant, which is a strong evidence that the effect of HF concentration on etch rate

is dependant on MTES concentration. Water has a negligible effect, which is consistent with the main effects estimates. 


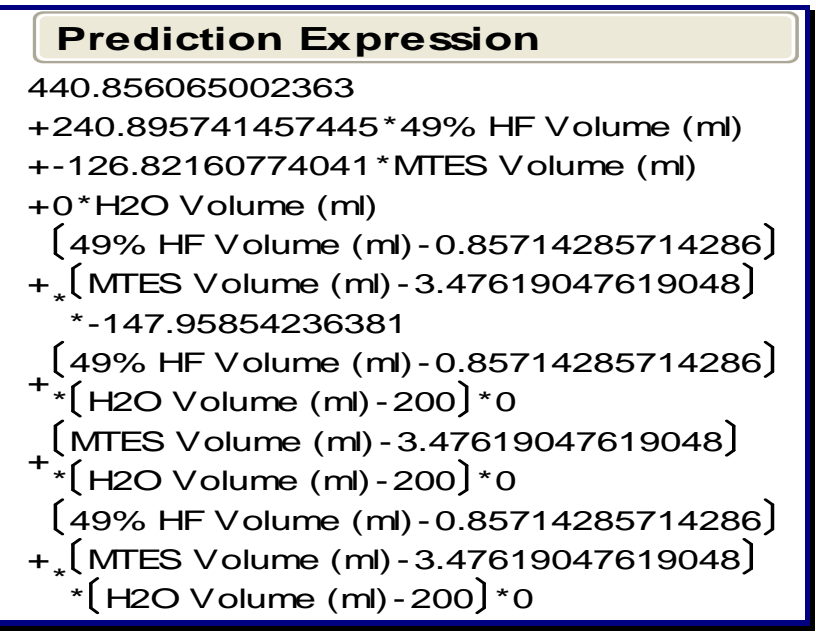

Figure 5.11 Ti etch rate prediction expression.

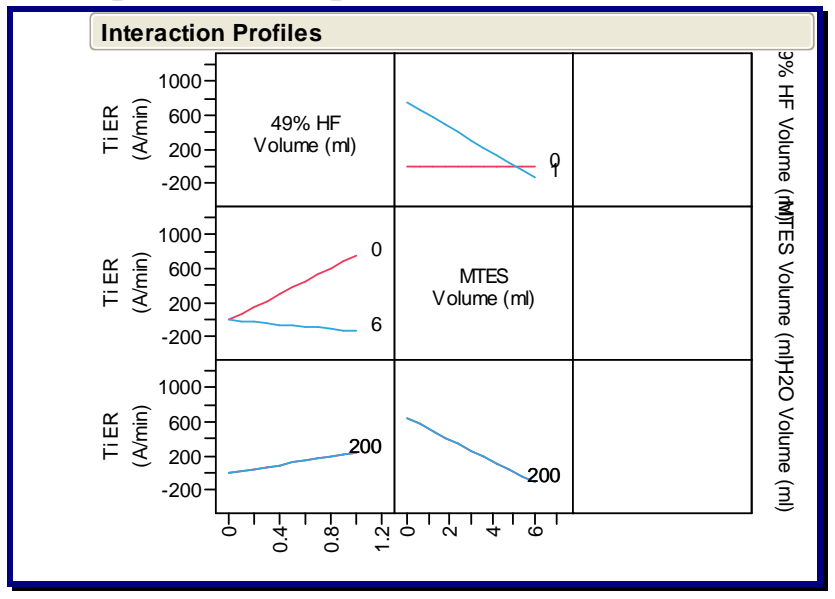

Figure 5.12 Ti etch rate interaction profiles. HF and MTES concentrations are represented by the $\mathrm{X}$-axis. The red curve corresponds to the low concentration of the complementary component and the blue curve corresponds to the higher concentration.

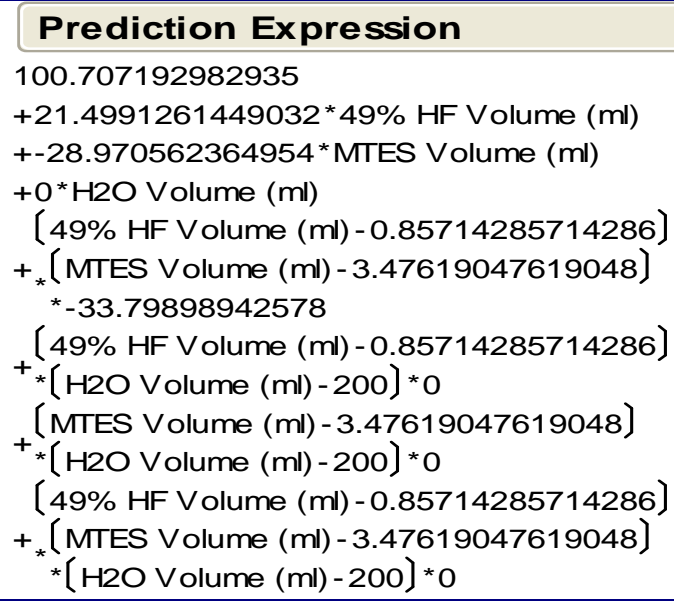

Figure 5.13 $\mathrm{SiO}_{x}$ etch rate prediction expression. 


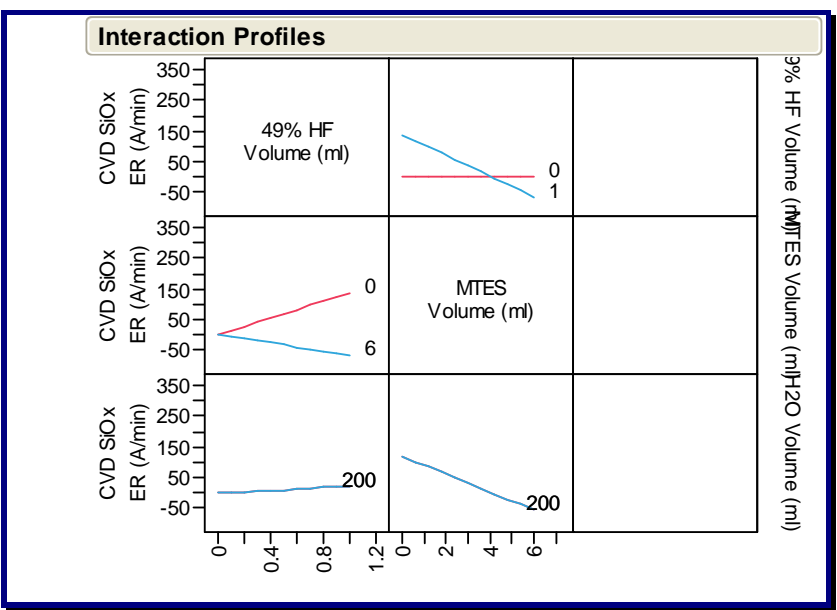

Figure 5.14 $\mathrm{SiO}_{\mathrm{x}}$ etch rate interaction profiles. $\mathrm{HF}$ and MTES concentrations are represented by the $\mathrm{X}$-axis. The red curve corresponds to the low concentration of the complementary component and the blue curve corresponds to the higher concentration.

$$
\begin{aligned}
& \text { Prediction Expression } \\
& 3.45321772076519 \\
& +0.74659179621521 * 49 \% \text { HF Volume }(\mathrm{ml}) \\
& +-0.9933913991242 * \text { MTES Volume }(\mathrm{ml}) \\
& +0{ }^{*} \mathrm{H} 2 \mathrm{O} \text { Volume }(\mathrm{ml}) \\
& {[49 \% \text { HF Volume }(\mathrm{ml})-0.85714285714286]} \\
& +{ }_{*}[\text { MTES Volume }(\mathrm{ml})-3.47619047619048] \\
& { }^{*}-1.1589566323116 \\
& {[49 \% \text { HF Volume }(\mathrm{ml})-0.85714285714286]} \\
& +{ }_{*}[\text { H2O Volume }(\mathrm{ml})-200] * 0 \\
& {[\text { MTES Volume }(\mathrm{ml})-3.47619047619048)} \\
& +{ }_{*}[\text { H2O Volume }(\mathrm{ml})-200] * 0 \\
& {[49 \% \text { HF Volume }(\mathrm{ml})-0.85714285714286]} \\
& +{ }_{*}[\text { MTES Volume }(\mathrm{ml})-3.47619047619048] \\
& *[\text { H2O Volume }(\mathrm{ml})-200] * 0
\end{aligned}
$$

Figure 5.15 $\mathrm{Si}_{3} \mathrm{~N}_{4}$ etch rate prediction expression. 


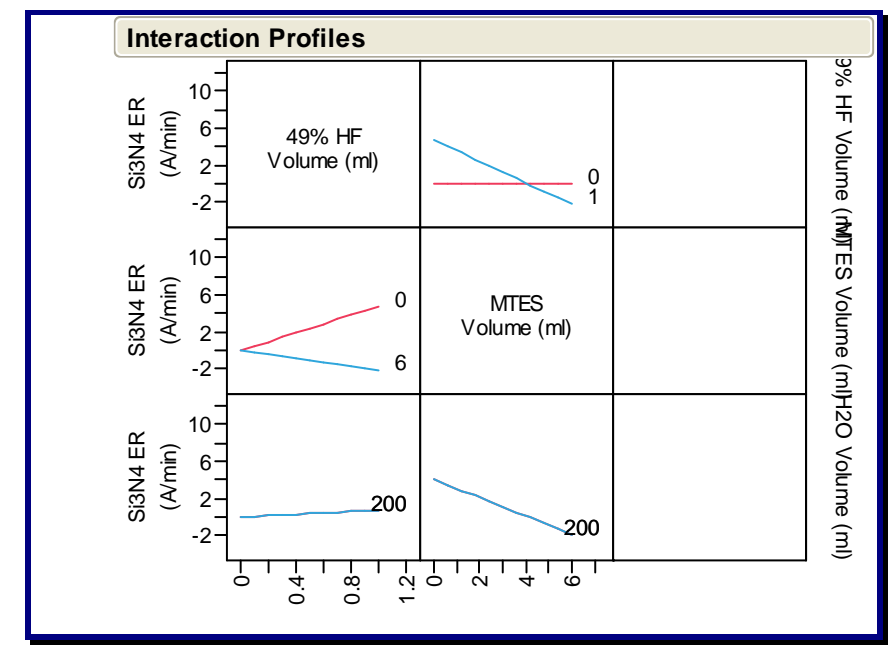

Figure 5.16 $\mathbf{S i}_{3} \mathbf{N}_{4}$ etch rate interaction profiles. HF and MTES concentrations are represented by the $\mathrm{X}$-axis. The red curve corresponds to the low concentration of the complementary component and the blue curve corresponds to the higher concentration.

\subsubsection{Prediction and Contour Profiler for $\mathrm{Ti}, \mathrm{SiO}_{\mathrm{x}}$, and $\mathrm{Si}_{3} \mathrm{~N}_{4}$ etch rates}

Profiling is an easy way to visualize a response surface. The prediction profiler depicted in Figure 5.17, shows vertical slices across each factor HF, MTES, and water concentrations, while holding other factors at current values. In this analysis, the software (JMP) computes a grid of predicted values of etch rates and desirability for each predicted value within the experimental region. 


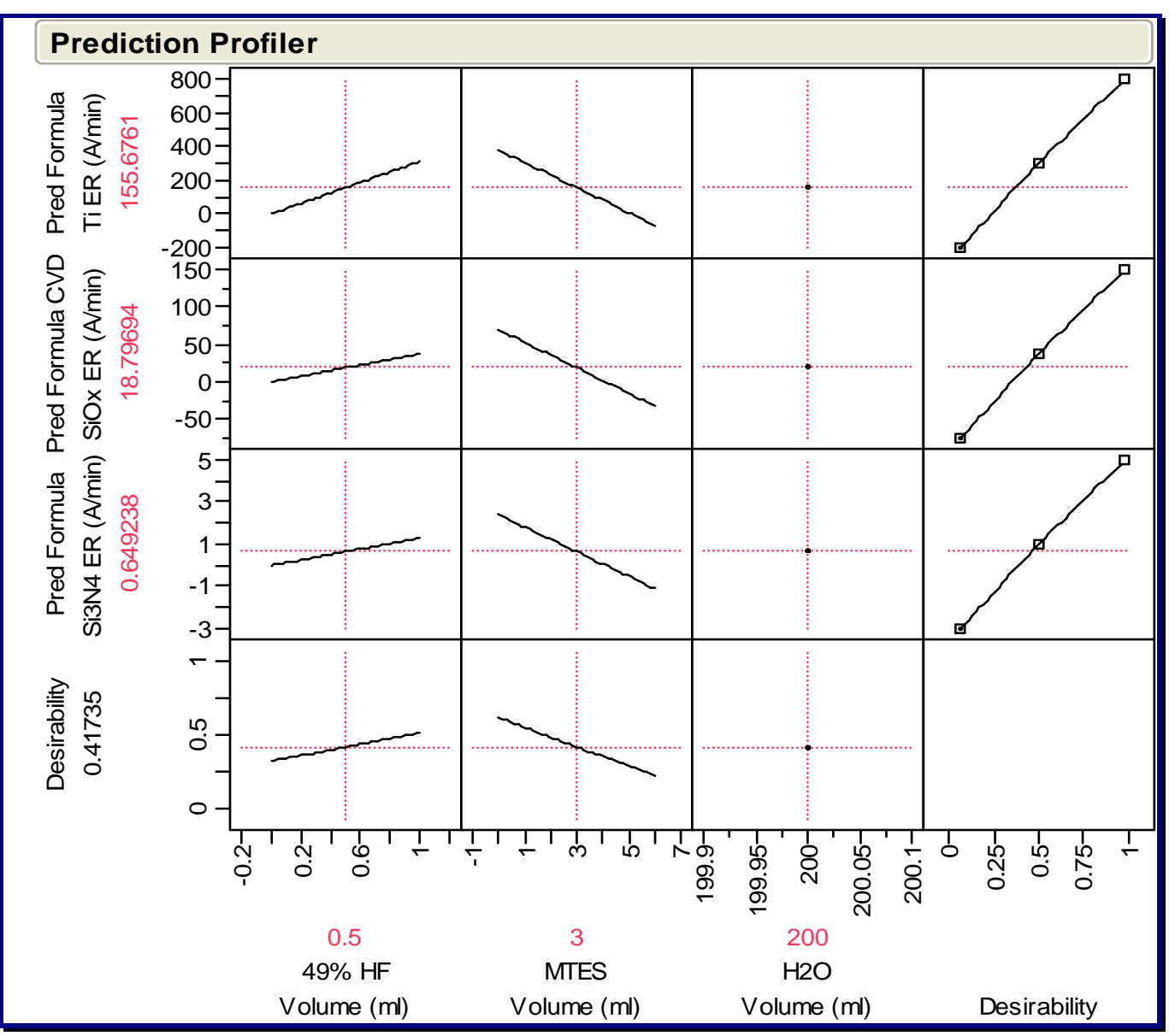

Figure 5.17 Prediction Profiler for $\mathrm{Ti}, \mathrm{SiO}_{\mathrm{x}}$, and $\mathrm{Si}_{3} \mathrm{~N}_{4}$ etch rates from the fitted model.

The ultimate goal of the factorial DOE is to pinpoint a formulation composition target and operating window also known as the feasible region, to provide the most optimal etch rates as defined by the success criterions. A contour profiler approach was employed in which the factorial DOE analysis and fitted models were used to locate the process target and window.

A contour profiler is shown in Figure 5.18 in which etch rate contours and response surfaces as a function of $\mathrm{HF}$ and MTES concentrations are illustrated. The 
contour profiler shows response contours for two factors at a time. This is a useful technique to optimize response surfaces graphically.

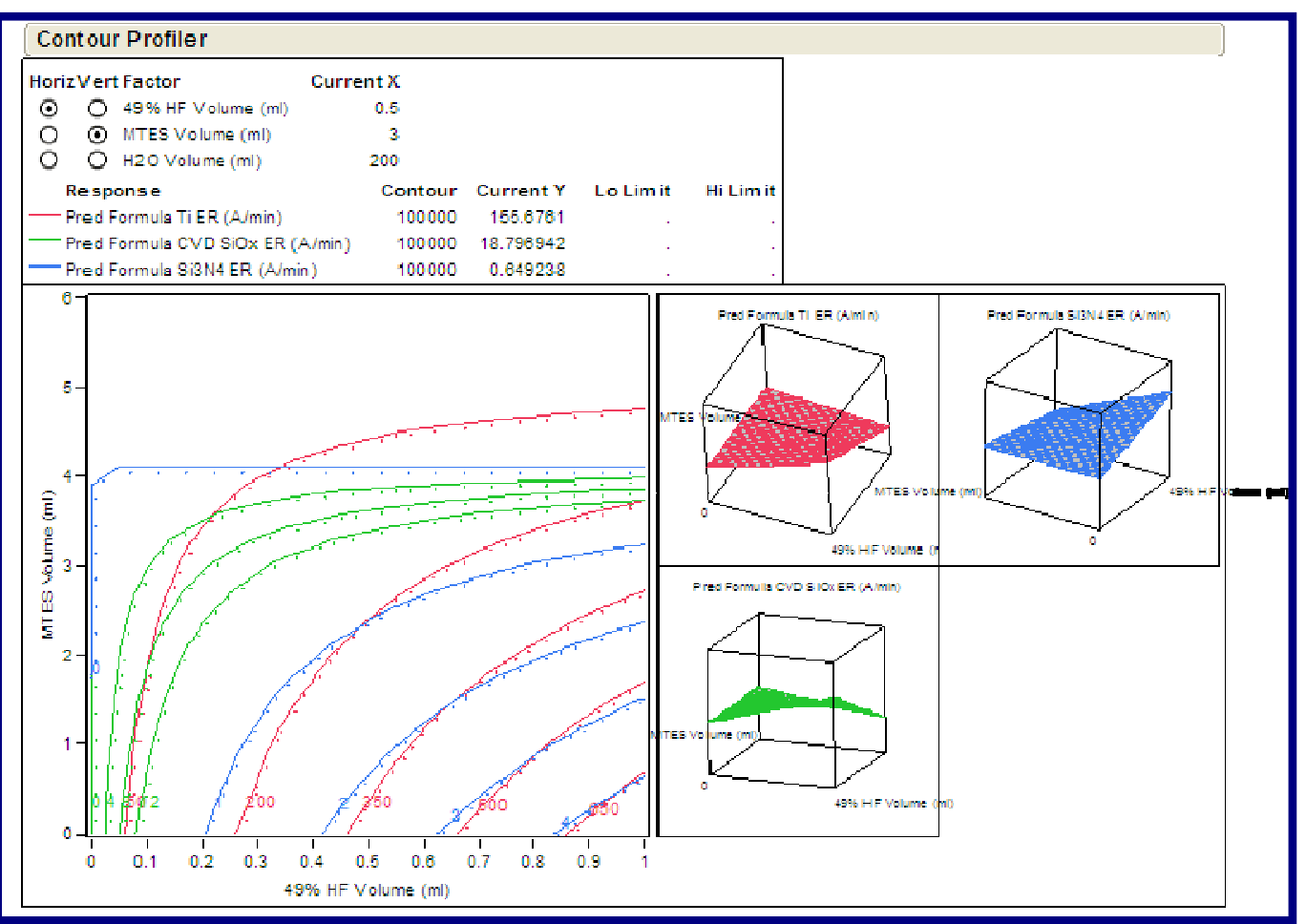

Figure 5.18 Etch rate DOE/response surface for HF, MTES, and water concentrations.

Figure 5.19 shows an acceptable process window with $\mathrm{Ti}, \mathrm{SiO}_{\mathrm{x}}$, and $\mathrm{Si}_{3} \mathrm{~N}_{4}$ etch rates between 50-400, 0-5, and 0-5 $\mathrm{A} \%$ min respectively. The unshaded (white) region in the larger plot includes HF and MTES concentrations where the predicted etch rates satisfies the etch rate limits requirement. The 3-D contour plots illustrate the fitted "response surface" (i.e., fitted model) for each response. 


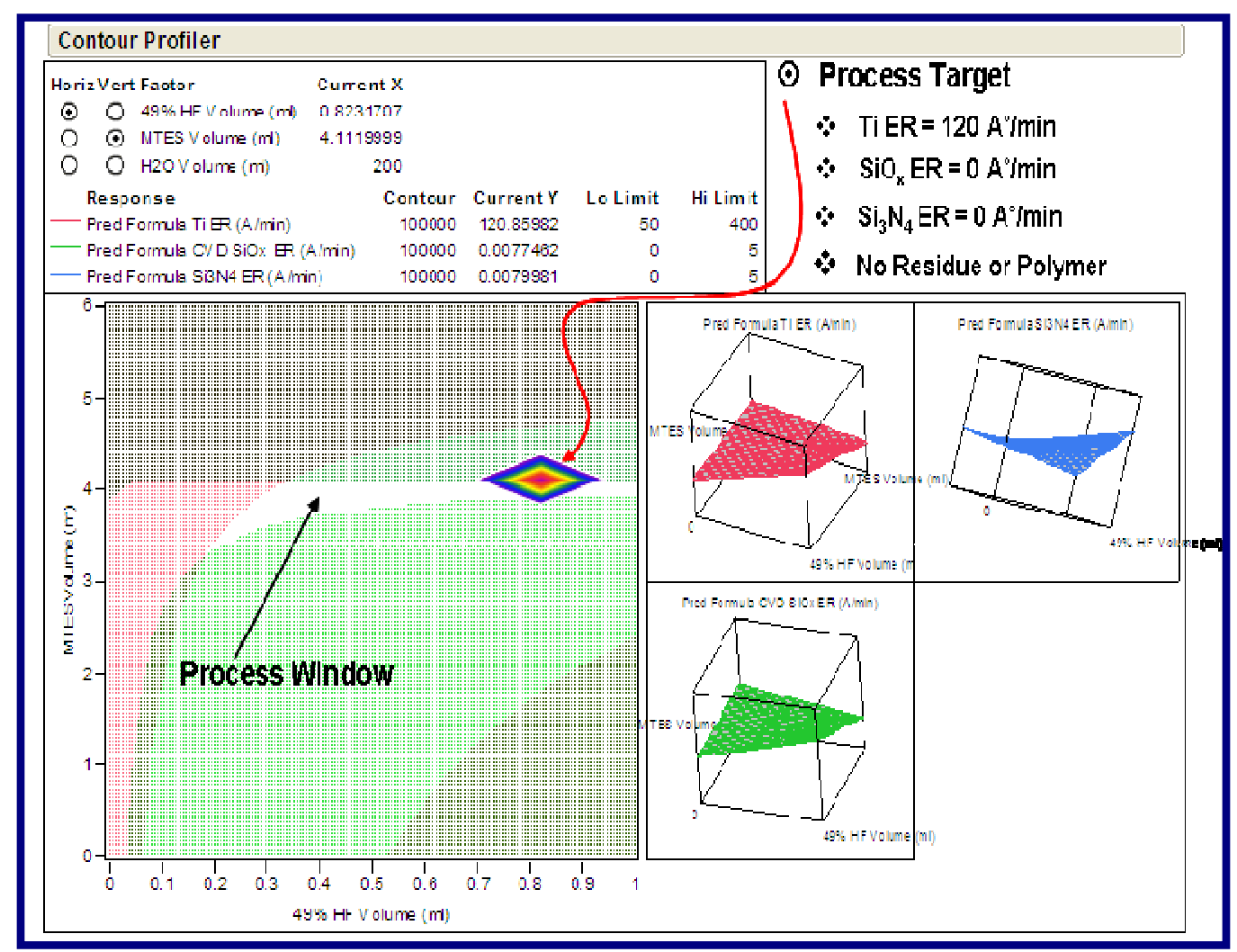

Figure 5.19 Etch rate DOE showing process target and process window

Table 5.2 is populated with etch rate data based on the DOE model and additional performed experiments. Note etch rates can be maintained invariable by holding the ratio of HF to MTES constant as long as water is in excess. This is an indication of the importance of the HF/MTES ratio as a critical parameter in synthesizing the formulation. 
Table 5.2 Etch rates as a function of concentrations by volume and weight $\%$.

\begin{tabular}{|c|c|c|c|c|c|c|c|c|c|c|c|}
\hline $\begin{array}{c}\mathrm{HF} \\
\text { Concentration } \\
(w t \%)\end{array}$ & $\begin{array}{c}\text { MTES } \\
\text { Concentration } \\
\text { (wt } \%)\end{array}$ & $\begin{array}{c}\mathrm{H} 2 \mathrm{O} \\
\text { Concentration } \\
(\mathrm{wt} \%)\end{array}$ & $\begin{array}{c}49 \% \mathrm{HF} \\
\text { Volume } \\
(\mathrm{ml})\end{array}$ & $\begin{array}{l}\text { MTES } \\
\text { Volume } \\
\text { (ml) }\end{array}$ & $\begin{array}{l}\mathrm{H} 2 \mathrm{O} \\
\text { Volume } \\
\text { (ml) }\end{array}$ & $\begin{array}{c}\text { Ti ER } \\
\text { (A/min) }\end{array}$ & $\begin{array}{c}\text { CVD } \\
\text { SiOx ER } \\
\text { (A/min) }\end{array}$ & $\begin{array}{c}\text { Si3N4 } \\
\text { ER } \\
(\mathrm{A} / \mathrm{min})\end{array}$ & $\begin{array}{l}\text { W ER } \\
(A / \min )\end{array}$ & $\begin{array}{c}\text { CDO ER } \\
(\mathrm{A} / \mathrm{min})\end{array}$ & $\begin{array}{l}\mathrm{SiC} E R \\
(\mathrm{~A} / \mathrm{min})\end{array}$ \\
\hline 0.237 & 2.602 & 97.160 & 1 & 6 & 200 & 24.000 & -1 & 0.000 & 0 & 0 & 0 \\
\hline 0.237 & 2.602 & 97.160 & 1 & 6 & 200 & 24.000 & -1.1 & 0.000 & 0 & 0 & 0 \\
\hline 0.238 & 2.390 & 97.372 & 1 & 5.5 & 200 & 30.738 & -0.9 & 0.000 & 0 & 0 & 0 \\
\hline 0.238 & 2.390 & 97.372 & 1 & 5.5 & 200 & 30.738 & -0.8 & 0.000 & 0 & 0 & 0 \\
\hline 0.238 & 2.178 & 97.584 & 1 & 5 & 200 & 43.103 & -0.6 & 0.000 & 0 & 0 & 0 \\
\hline 0.238 & 2.178 & 97.584 & 1 & 5 & 200 & 43.103 & -0.6 & 0.000 & 0 & 0 & 0 \\
\hline 0.239 & 1.964 & 97.797 & 1 & 4.5 & 200 & 56.604 & -0.1 & 0.000 & 0 & 0 & 0 \\
\hline 0.239 & 1.964 & 97.797 & 1 & 4.5 & 200 & 56.604 & -0.2 & 0.000 & 0 & 0 & 0 \\
\hline 0.240 & 1.750 & 98.011 & 1 & 4 & 200 & 100.000 & 0 & 0.000 & 0 & 0 & 0 \\
\hline 0.240 & 1.750 & 98.011 & 1 & 4 & 200 & 100.000 & 0 & 0.000 & 0 & 0 & 0 \\
\hline 0.240 & 1.664 & 98.096 & 1 & 3.8 & 200 & 120.000 & 0 & 0.000 & 0 & 0 & 0 \\
\hline 0.240 & 1.664 & 98.096 & 1 & 3.8 & 200 & 120.000 & 0 & 0.000 & 0 & 0 & 0 \\
\hline 0.240 & 1.664 & 98.096 & 1 & 3.8 & 200 & 120.000 & 0 & 0.000 & 0 & 0 & 0 \\
\hline 0.240 & 1.578 & 98.182 & 1 & 3.6 & 200 & 148.515 & 0.4 & 0.014 & 0 & 0 & 0 \\
\hline 0.240 & 1.578 & 98.182 & 1 & 3.6 & 200 & 148.515 & 0.4 & 0.014 & 0 & 0 & 0 \\
\hline 0.240 & 1.491 & 98.269 & 1 & 3.4 & 200 & 176.471 & 1.1 & 0.038 & 0 & 0 & 0 \\
\hline 0.240 & 1.491 & 98.269 & 1 & 3.4 & 200 & 180.723 & 1.2 & 0.041 & 0 & 0 & 0 \\
\hline 0.240 & 1.448 & 98.312 & 1 & 3.3 & 200 & 254.237 & 2.6 & 0.090 & 0 & 0 & 0 \\
\hline 0.240 & 1.448 & 98.312 & 1 & 3.3 & 200 & 254.237 & 2.6 & 0.090 & 0 & 0 & 0 \\
\hline 0.240 & 1.405 & 98.355 & 1 & 3.2 & 200 & 272.727 & 3.1 & 0.107 & 0 & 0 & 0 \\
\hline 0.240 & 1.405 & 98.355 & 1 & 3.2 & 200 & 267.857 & 3.1 & 0.107 & 0 & 0 & 0 \\
\hline 0.241 & 1.318 & 98.441 & 1 & 3 & 200 & 288.462 & 3.7 & 0.128 & 0 & 0 & 0 \\
\hline 0.241 & 1.318 & 98.441 & 1 & 3 & 200 & 288.462 & 3.8 & 0.131 & 0 & 0 & 0 \\
\hline 0.241 & 1.231 & 98.528 & 1 & 2.8 & 200 & 306.122 & 4.5 & 0.155 & 0 & 0 & 0 \\
\hline 0.241 & 1.231 & 98.528 & 1 & 2.8 & 200 & 306.122 & 4.5 & 0.155 & 0 & 0 & 0 \\
\hline 0.241 & 1.144 & 98.615 & 1 & 2.6 & 200 & 326.087 & 5.6 & 0.193 & 0 & 0 & 0 \\
\hline 0.241 & 1.144 & 98.615 & 1 & 2.6 & 200 & 333.333 & 5.6 & 0.193 & 0 & 0 & 0 \\
\hline 0.241 & 1.057 & 98.701 & 1 & 2.4 & 200 & 357.143 & 6.8 & 0.234 & 0 & 0 & 0 \\
\hline 0.241 & 1.057 & 98.701 & 1 & 2.4 & 200 & 357.143 & 6.8 & 0.234 & 0 & 0 & 0 \\
\hline 0.469 & 3.258 & 96.273 & 1 & 3.8 & 100 & 122.951 & 0 & 0.000 & 0 & 0 & 0 \\
\hline 0.469 & 3.258 & 96.273 & 1 & 3.8 & 100 & 123.967 & 0 & 0.000 & 0 & 0 & 0 \\
\hline 0.471 & 2.841 & 96.687 & 1 & 3.3 & 100 & 263.158 & 2.7 & 0.093 & 0 & 0 & 0 \\
\hline 0.471 & 2.841 & 96.687 & 1 & 3.3 & 100 & 263.158 & 2.7 & 0.093 & 0 & 0 & 0 \\
\hline 0.761 & 5.281 & 93.958 & 1 & 3.8 & 60 & 126.050 & 0 & 0.000 & 0 & 0 & 0 \\
\hline 0.761 & 5.281 & 93.958 & 1 & 3.8 & 60 & 127.119 & 0 & 0.000 & 0 & 0 & 0 \\
\hline 0.766 & 4.618 & 94.616 & 1 & 3.3 & 60 & 263.158 & 2.8 & 0.097 & 0 & 0 & 0 \\
\hline 0.766 & 4.618 & 94.616 & 1 & 3.3 & 60 & 263.158 & 2.8 & 0.097 & 0 & 0 & 0 \\
\hline 1.104 & 7.660 & 91.237 & 1 & 3.8 & 40 & 130.435 & 0.2 & 0.007 & 0 & 0 & 0 \\
\hline 1.104 & 7.660 & 91.237 & 1 & 3.8 & 40 & 130.435 & 0.2 & 0.007 & 0 & 0 & 0 \\
\hline 1.110 & 7.098 & 91.792 & 1 & 3.5 & 40 & 227.273 & 1.2 & 0.041 & 0 & 0 & 0 \\
\hline 1.110 & 7.098 & 91.792 & 1 & 3.5 & 40 & 227.273 & 1.2 & 0.041 & 0 & 0 & 0 \\
\hline 1.115 & 6.720 & 92.166 & 1 & 3.3 & 40 & 267.857 & 2.9 & 0.100 & 0 & 0 & 0 \\
\hline 1.115 & 6.720 & 92.166 & 1 & 3.3 & 40 & 272.727 & 2.9 & 0.100 & 0 & 0 & 0 \\
\hline 2.008 & 13.938 & 84.054 & 1 & 3.8 & 20 & 141.509 & 1.3 & 0.045 & 0 & 0 & 0 \\
\hline 2.008 & 13.938 & 84.054 & 1 & 3.8 & 20 & 144.231 & 1.4 & 0.048 & 0 & 0 & 0 \\
\hline 2.046 & 12.330 & 85.624 & 1 & 3.3 & 20 & 306.122 & 3.6 & 0.124 & 0 & 0 & 0 \\
\hline 2.046 & 12.330 & 85.624 & 1 & 3.3 & 20 & 312.500 & 3.8 & 0.131 & 0 & 0 & 0 \\
\hline 0.049 & 0.000 & 99.951 & 1 & 0 & 1000 & 348.837 & 66 & 2.276 & 0 & 0 & 0 \\
\hline 0.098 & 0.000 & 99.902 & 1 & 0 & 500 & 555.556 & 133 & 4.586 & 0 & 0 & 0 \\
\hline 0.122 & 0.000 & 99.878 & 1 & 0 & 400 & 625.000 & 151 & 5.207 & 0 & 0 & 0 \\
\hline 0.163 & 0.000 & 99.837 & 1 & 0 & 300 & 789.474 & 227 & 7.828 & 0 & 0 & 0 \\
\hline 0.240 & 0.000 & 99.760 & 1 & 0 & 200 & 1000.000 & 306 & 10.552 & 0 & 0 & 0 \\
\hline 0.490 & 0.000 & 99.510 & 1 & 0 & 100 & 1666.667 & 611 & 21.069 & 0 & 0 & 0 \\
\hline 0.980 & 0.000 & 99.020 & 1 & 0 & 50 & 2500.000 & 1221 & 42.103 & 0 & 0 & 0 \\
\hline 0.000 & 2.615 & 97.385 & 0 & 6 & 200 & 0.000 & 0 & 0.000 & 0 & 0 & 0 \\
\hline 0.000 & 1.325 & 98.675 & 0 & 3 & 200 & 0.000 & 0 & 0.000 & 0 & 0 & 0 \\
\hline 0.000 & 0.000 & 100.000 & 0 & 0 & 200 & 0.000 & 0 & 0.000 & 0 & 0 & 0 \\
\hline 0.000 & 0.000 & 100.000 & 0 & 0 & 100 & 0.000 & 0 & 0.000 & 0 & 0 & 0 \\
\hline
\end{tabular}




\subsection{Enabling the Formulation for Copper}

So far, the work described earlier deals with the development of a novel formulation that can be used at the first interconnect layer to remove a Ti hard-mask after DD steps in which tungsten becomes exposed during the Ti stripping process. To further enable the formulation for all interconnect layers, it must exhibit compatibility towards copper. The strategy of employing organic copper corrosion inhibitors was the leading approach to alleviate copper corrosion and protect the copper surface during the Ti strip process. From a practical point of view, Benzotriazole (BTA) was selected for this application. The goal of this work is to determine optimal BTA concentration needed to provide a robust passivation to the copper surface during the wet etch clean process, and ensure that BTA has no adverse impact on copper interconnects in patterned DD structures, in particular.

The copper industry has used BTA as a corrosion inhibitor for more than 50 years $^{212}$. It is a common corrosion inhibitor, its role is overwhelming, and consequently it has been studied in detail. It is known that copper forms a cuprous benzotriazole complex, $[\mathrm{Cu}(\mathrm{I})-\mathrm{BTA}]$, which is responsible for the corrosion inhibition ${ }^{213,214,215,216,217}$. Today it is widely accepted that it forms a complex layer with $\mathrm{Cu}-\mathrm{I}$ ions on the copper surface using $\mathrm{Cu}-\mathrm{N}$ bond ${ }^{218}$ and that it is an anodic-type inhibitor. An illustration of BTA adsorption onto the $\mathrm{Cu}$ surface is shown in Figure $5.20^{219}$ 


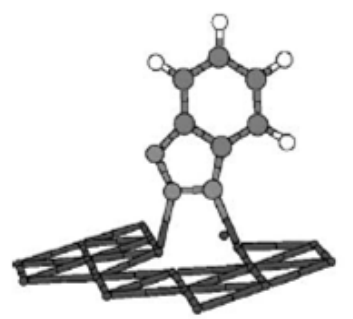

(a) $\Delta \mathrm{H}=-0.407 \mathrm{eV}$

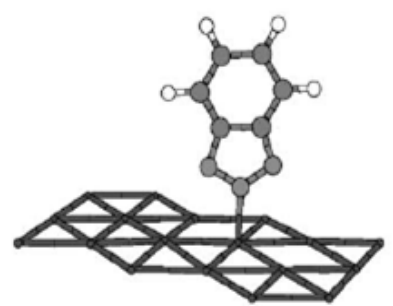

(b) $\Delta \mathrm{H}=+0.133 \mathrm{eV}$

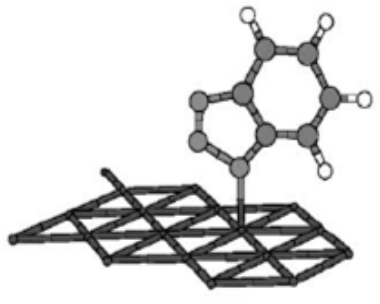

(c) not stable, finally converge to (a)

Figure 5.20 Three adsorption coordination of BTA. Only the first layer is shown.

The copper corrosion characterization and inhibition study is herein discussed.

This investigation includes an electrochemical analysis, in which equilibrium rest potential (Open Circuit Potential), linear polarization and Tafel polarization techniques were carried out to determine the corrosion rate in the presence of BTA. The electrochemical experiments were performed on an organic acid (2\% citric acid solution) stripped copper samples. Surface analysis using SEM were performed before and after immersion in the formulation solution to investigate the phenomenon of pitting corrosion.

\subsubsection{Copper Corrosion Results}

The open circuit potential study is illustrated in Figure 5.21 indicates that the bulk of copper film is passivated at about 100 seconds in BTA range of $900-2000$ ppm. i.e., a protective film is formed on the surface and an equilibrium condition is reached. Figure 5.22 depicts the linear polarization measurements indicating negligible corrosion for BTA concentrations of $900-2000$ ppm. 


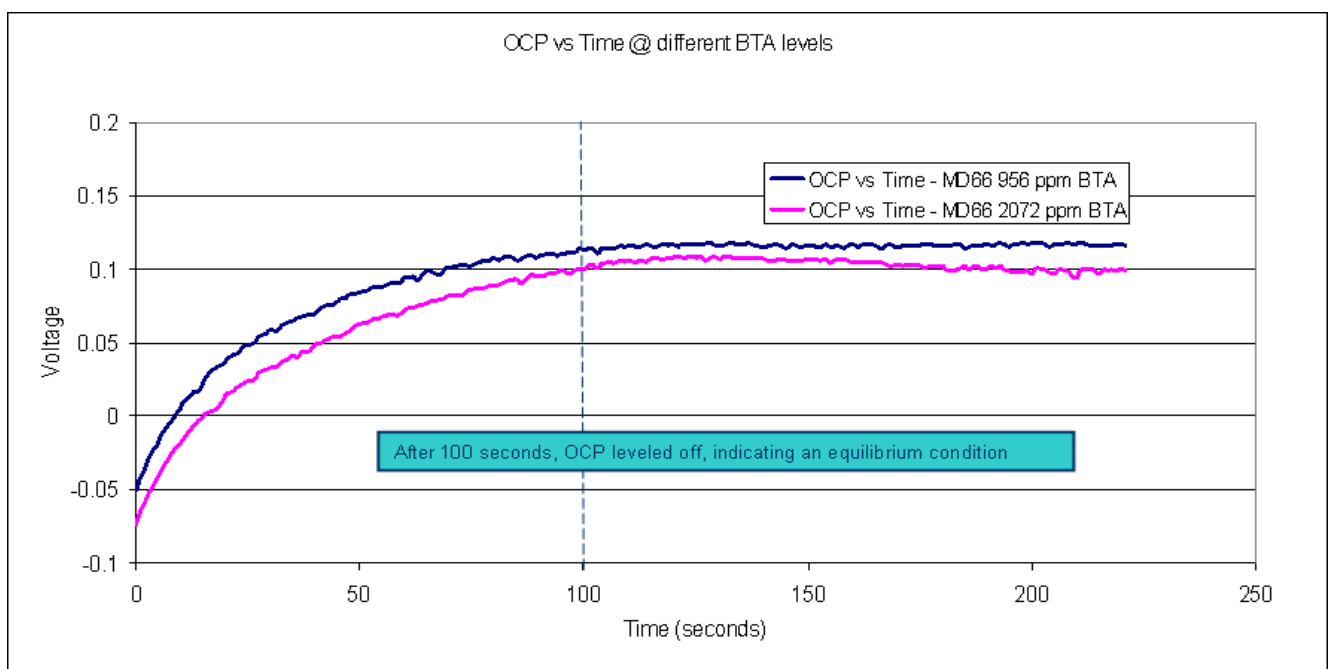

Figure 5.21 Rest potential for two different BTA concentrations.

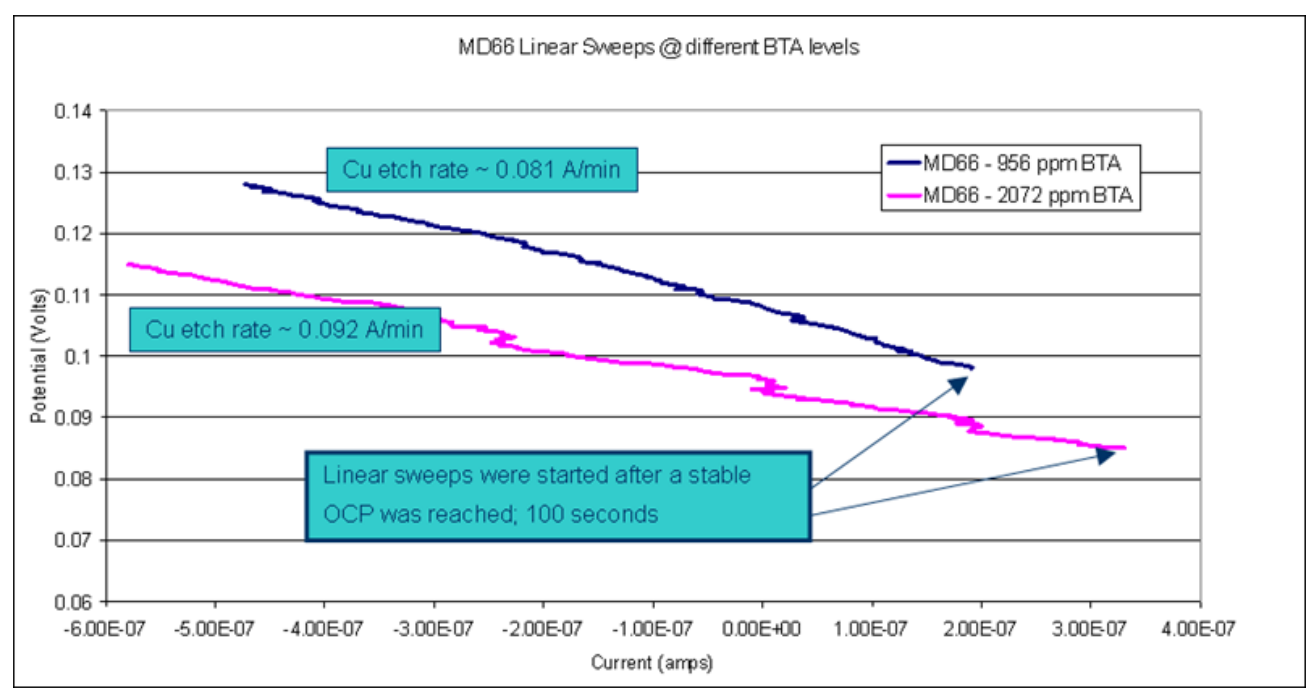

Figure 5.22 Linear Polarization measurements on $\mathrm{Cu}$ surface indicating very low corrosion.

The results of the potentiodynamic experiments (Tafel polarization) curves for copper obtained in the formulation solution are shown in Figure 5.23. The corrosion behavior of copper in the solution was modulated as a function of BTA concentration. The anodic polarization curves indicate negligible corrosion. The potentiodynamic polarization experiments were analyzed by employing Corrview ${ }^{\mathrm{TM}}$ software to determine corrosion rate in terms of corrosion current density $\left(\mathrm{I}_{\text {corr }}\right)$ using Tafel slopes. 
The corrosion rates for BTA concentrations of $900-2000 \mathrm{ppm}$ were $0.04 \mathrm{~A} \% \mathrm{~min}$. The surface characteristics of the copper thin film were investigated before and after processing in the wet etch formulation to ensure the absence of localized corrosion, such as Pitting corrosion. The SEM micrograph images shown in figure 5.24 did not reveal any indication of pit formation characteristic for pitting corrosion.

Although this investigation comprised BTA concentrations ranging from 900 $2000 \mathrm{ppm}$, a concentration of $800 \mathrm{ppm}$ offered a robust passivation to the surface and resulted with the same corrosion rate of $0.04 \mathrm{~A} / \mathrm{min}$. Therefore, a BTA concentration of $800 \mathrm{ppm}$ was incorporated into the formulation. High concentrations of BTA provided the necessary protection against corrosion. However, a white film Haze/residue forms on the copper surface when higher concentrations of BTA are used, in this formulation it was visible when $\sim 1300 \mathrm{ppm}$ is exceeded. This residue is visually observed and it stems from BTA's ability to form multi-layer film on the

copper surface ${ }^{220}$. The formulation was optimized in a manner where this issue is avoided. 


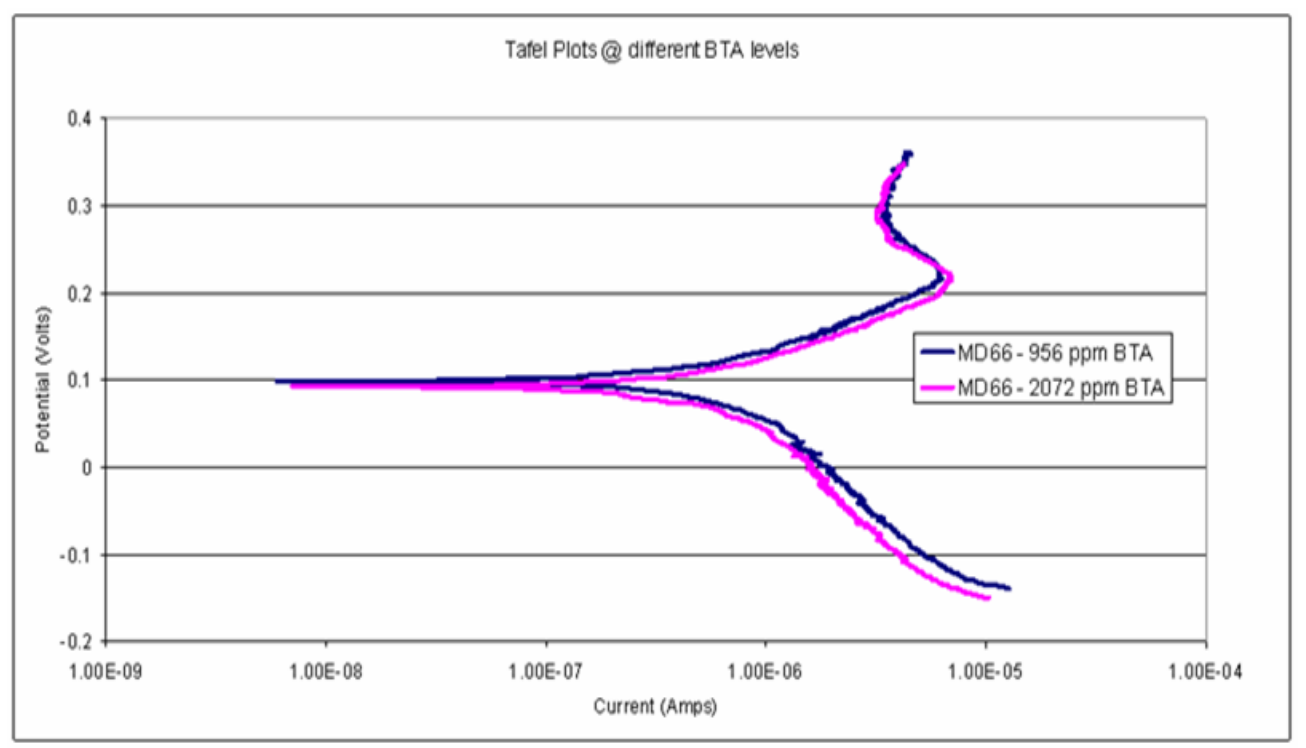

Figure 5.23 Tafel Polarization curves for copper thin films on $\mathrm{Si}$ in the formulation solution containing BTA. Copper etch rate $=0.04 \AA / \mathrm{min}$.

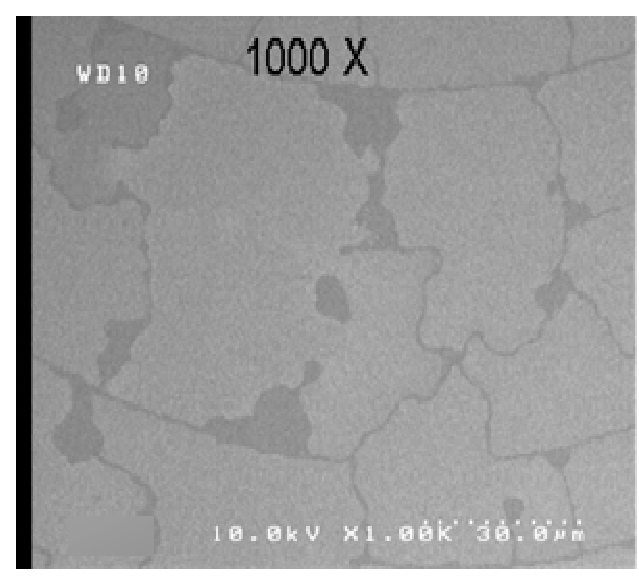

(a)

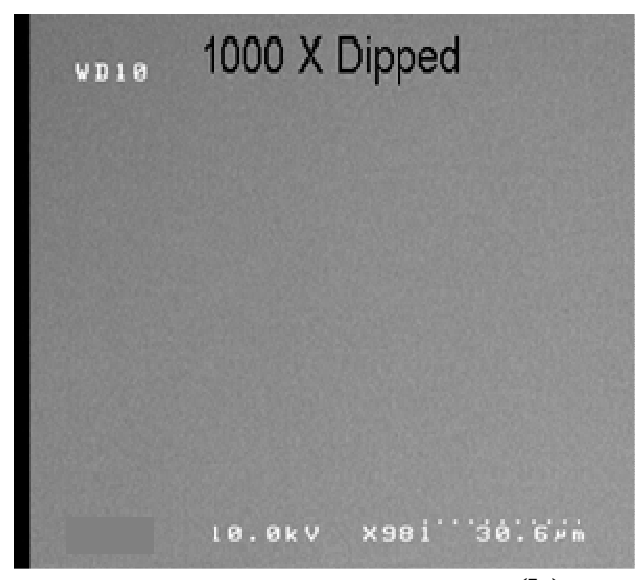

(b)

Figure 5.24 SEM micrograph of Copper surface before and after processing in the formulation in (a) and (b) respectively.

\subsection{Final Formulation Composition}

The final formulation composition and concentrations is illustrated in Table 5.3. The strategy and experimental work to reach this composition has been discussed in details in the previous sections. 
Table 5.3 Composition of the Ti selective wet etch formulation.

\begin{tabular}{|c|c|}
\hline Chemical & Concentration wt\% \\
\hline HF & 0.24 \\
\hline MTES & 1.66 \\
\hline BTA & 0.08 \\
\hline $\mathrm{H}_{2} \mathrm{O}$ & 98.02 \\
\hline
\end{tabular}

\subsection{Etch rates}

The etch rate results for the various substrates are shown in table 5.4 and Figure 5.25.

Table 5.4 Etch rate results of the various thin film materials in the Ti selective wet etch formulation at $60{ }^{\circ} \mathrm{C}$.

\begin{tabular}{|c|c|}
\hline Thin Film (Material) & Etch Rate @60 ${ }^{\circ} \mathrm{C}\left(A^{\circ} / \mathrm{min}\right)$ \\
\hline \hline $\mathrm{Ti}$ & 120 \\
\hline $\mathrm{W}$ & 0 \\
\hline $\mathrm{CVD} \mathrm{SiOx}$ & 0 \\
\hline $\mathrm{CDO}$ & 0 \\
\hline $\mathrm{Cu}$ & 0 \\
\hline Si3N4 & 0 \\
\hline SiC & 0 \\
\hline
\end{tabular}

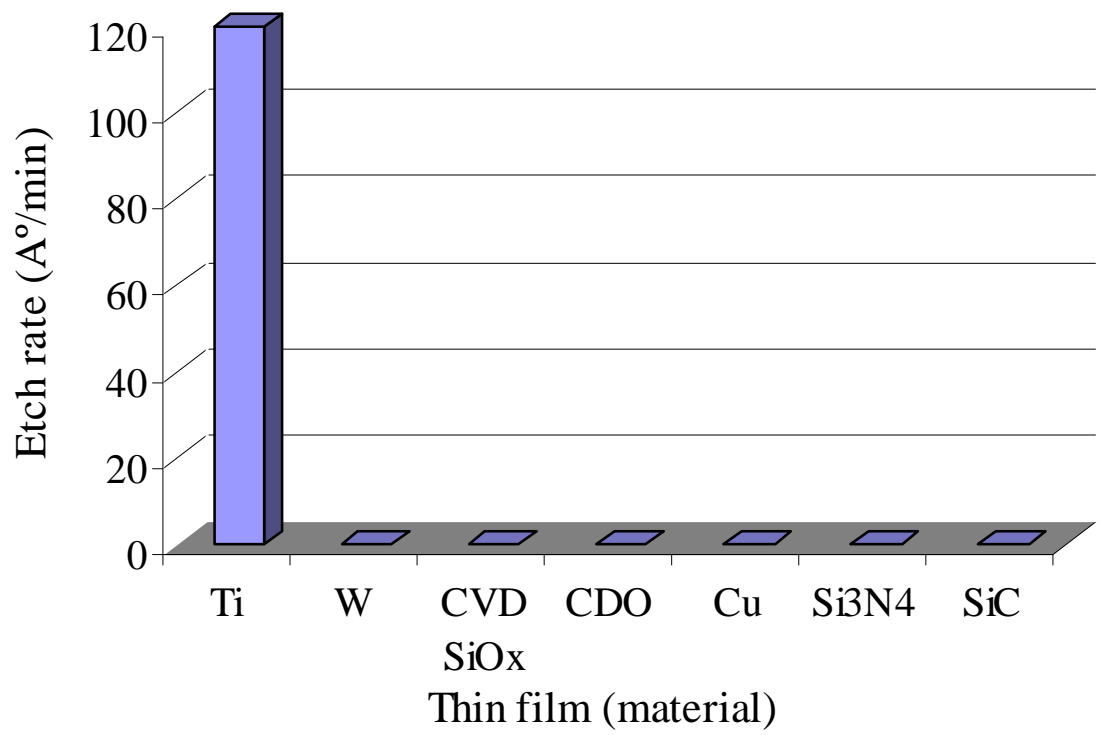

Figure 5.25 Etch rate results in the Ti selective wet etch formulation at $60{ }^{\circ} \mathrm{C}$. The maximum error in the ER determination is approximately $5 \%$. 


\subsection{Effects of Selective Wet Etch Chemistry on DD patterned wafers}

Figures 5.26 shows SEM micrograph of dual damascene patterned wafers before processing in the developed etch formulation solution.

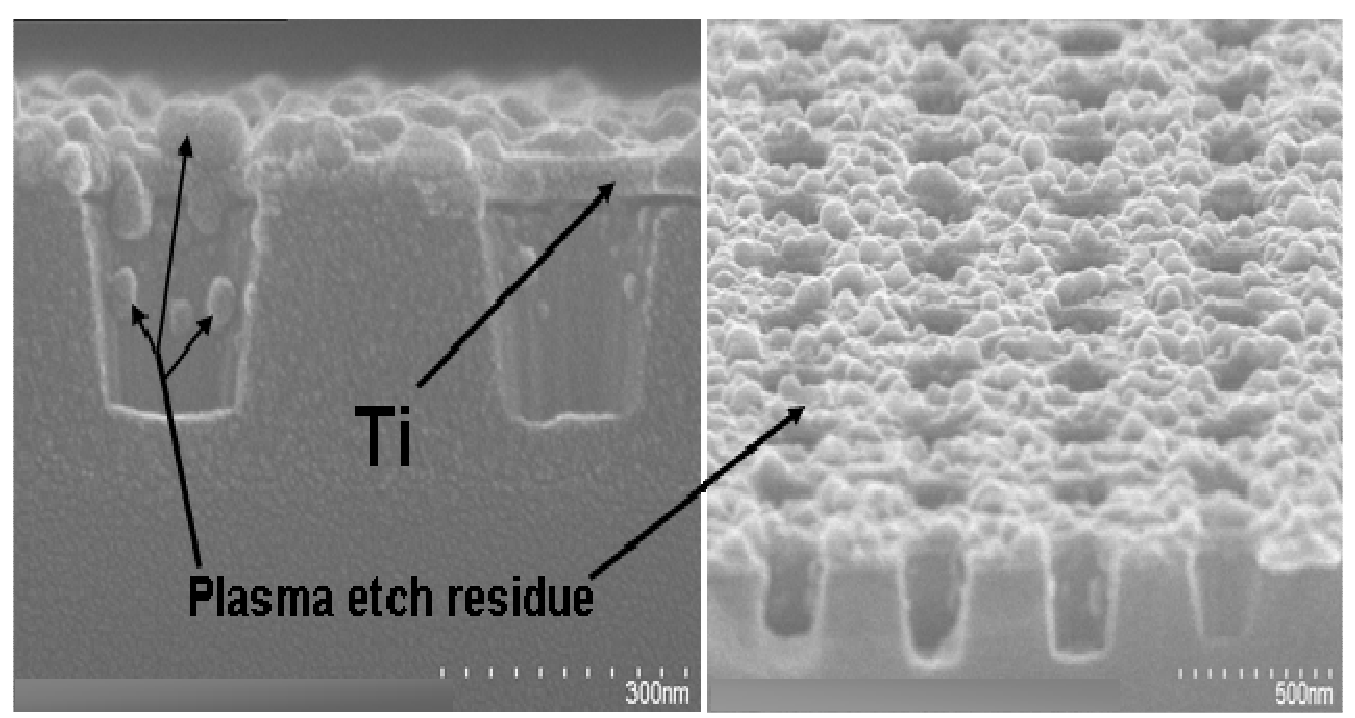

Figure 5.26 SEM micrograph of DD patterned wafers after the break-through plasma etch process and before treatment with the Ti selective wet etch chemistry.

Figure 5.27 shows SEM micrograph of DD patterned wafers after the break-through plasma etch process and after processing in the Ti selective etch formulation solution. Note the clean surface and complete removal of the plasma etch polymer/residue illustrated in (a). The SEM images shown in (b) and (C) reveal the formulations' compatibility towards $\mathrm{Cu}$ and $\mathrm{W}$ respectively. 


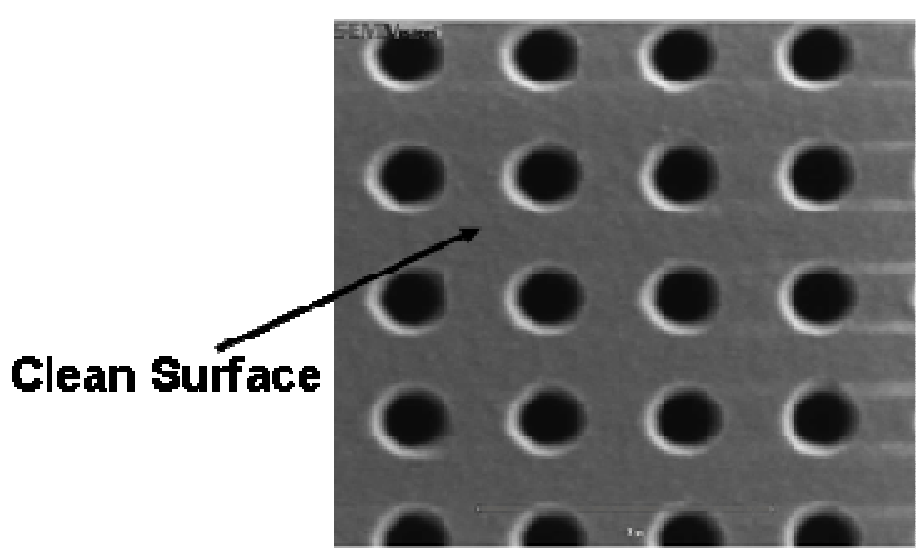

(a)

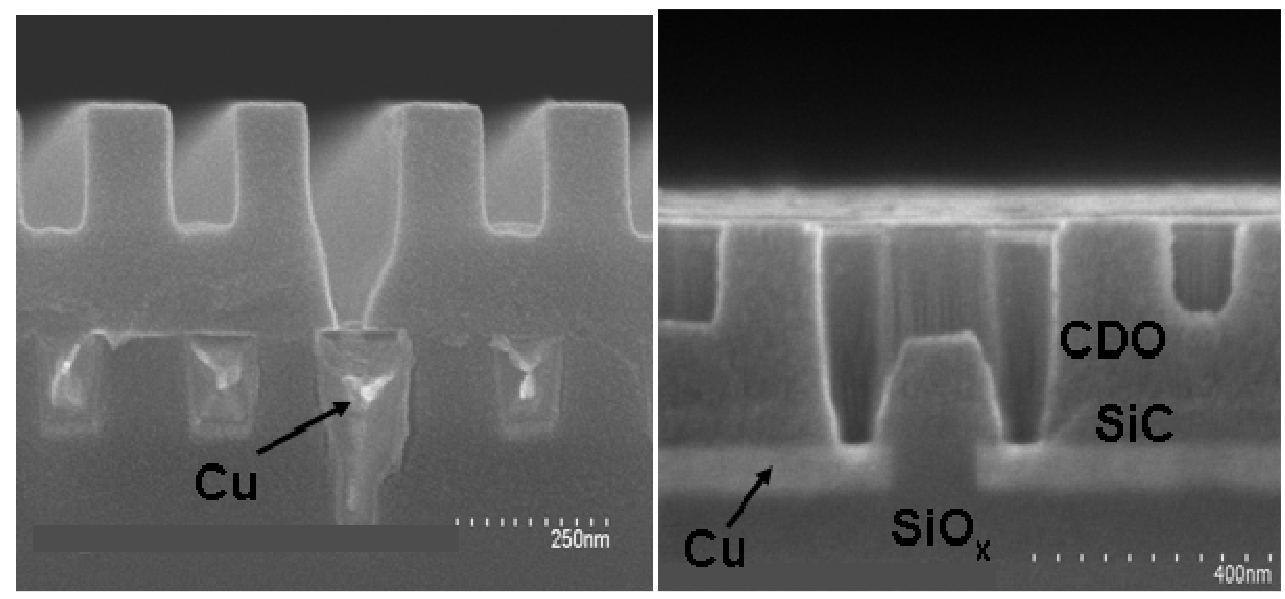

(b)
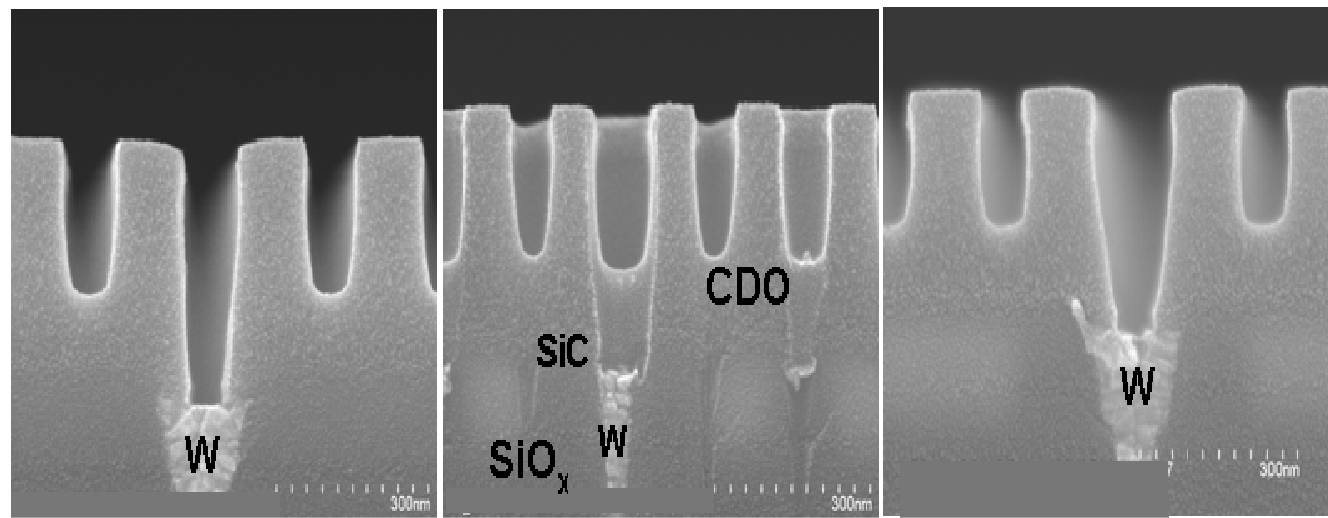

(c)

Figure 5.27 SEM micrograph of DD patterned wafers after the break-through plasma etch process and after treatment with the Ti selective wet etch chemistry. (a) illustrates the formulation's ability to remove the polymer/residue, (b), and (c) show it's compatibility towards copper and tungsten respectively. 


\subsection{Electrical performance}

Figure 5.28 shows the Resistance distribution of $\sim 65,000$ vias chain for advanced (new) and reference (prior) technology nodes. As previously stated, the developed selective wet etch/clean formulation is used for advanced process technology node, the previous technology distribution is shown as a reference for comparison. The electrical test results clearly show the lower resistance and narrower range for the advanced technology distribution.

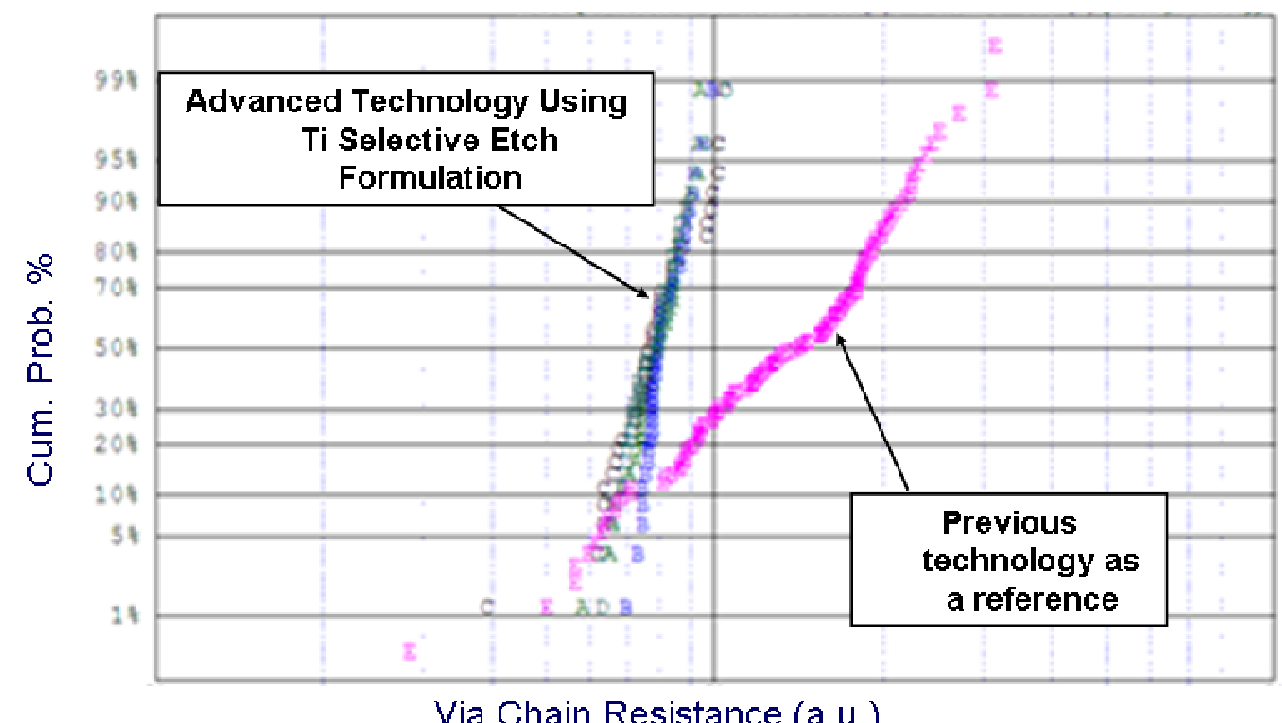

Figure 5.28 Resistance distribution of 65,000 vias chain for wafers cleaned with the developed $\mathrm{Ti}$ strip/clean chemistry. The reference distribution is shown for comparison.

\subsection{Time of Flight Secondary Ion Mass Spectrometry (TOF SIMS)}

The TOF SIMS data shown in Figure 5.29 indicate a complete removal of 250 Angstroms thick titanium film in 90 seconds. This result was further confirmed by 
optical microscopy studies as shown below in Figure 5.30 on blanket titanium wafers and in Figure 5.31 on patterned wafers.

Control (no etch) $30 \mathrm{sec}$ etch $60 \mathrm{sec}$ etch $73 \mathrm{sec}$ etch 75 sec etch

$90 \mathrm{sec}$ etch $105 \mathrm{sec}$ etch

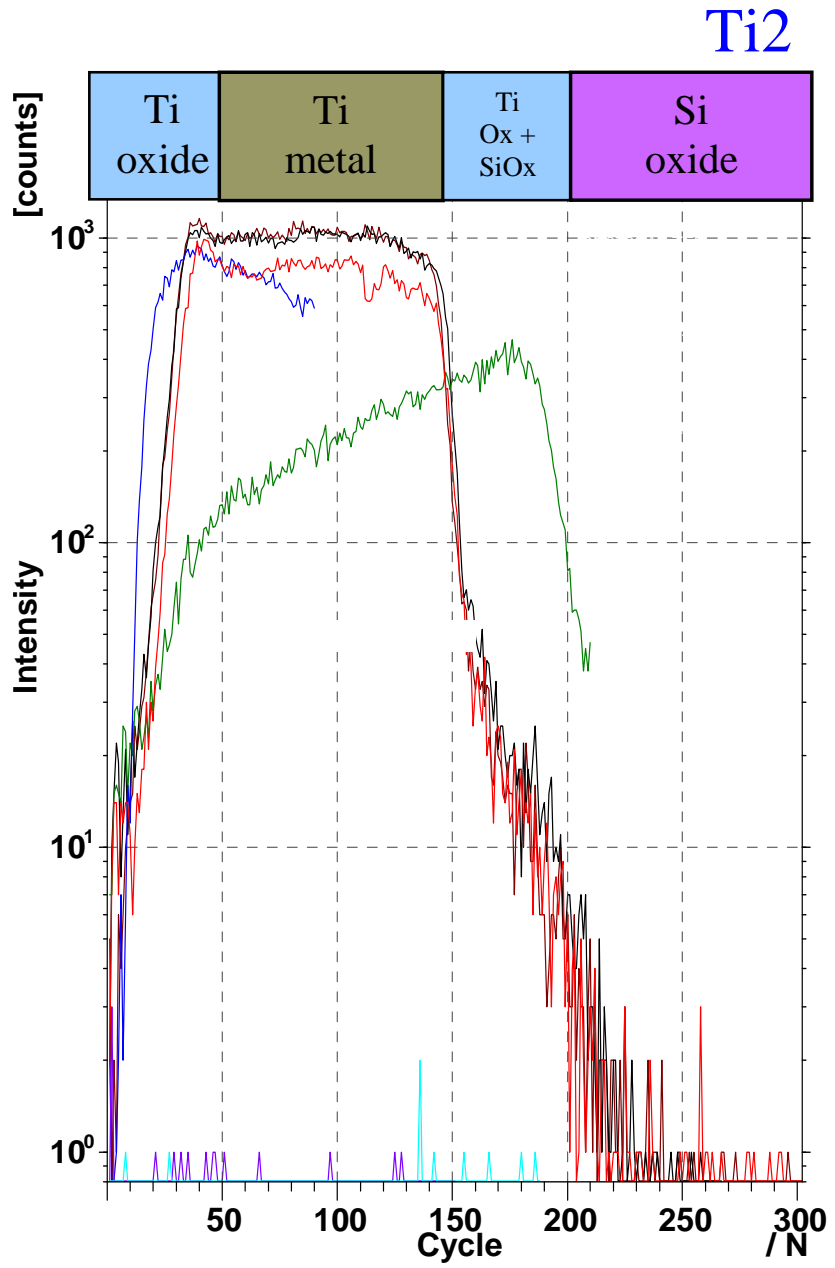

Figure 5.29 Depth profile determination of titanium concentration as a function of depth.

\section{Control}

(110 etch) $30 \mathrm{sec}$ etch $60 \mathrm{sec}$ etch $73 \mathrm{sec}$ etch $75 \mathrm{sec}$ etch $90 \mathrm{sec}$ etch $105 \mathrm{sec}$ etch

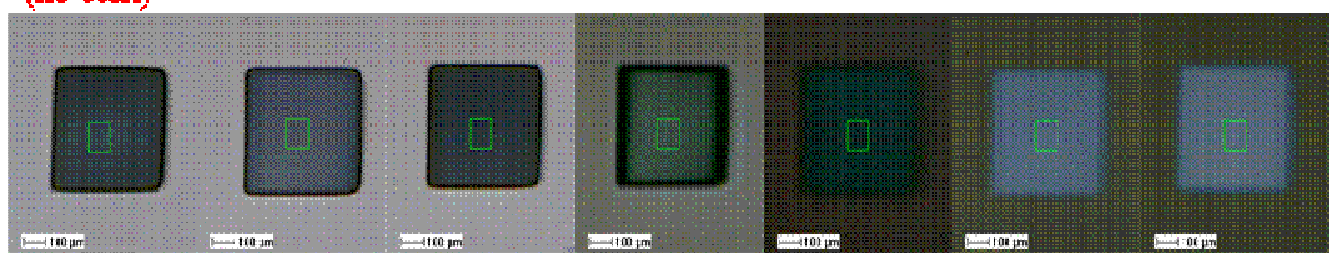

Figure 5.30 Evolution of reflectance images as a function of etch time. 
SRAM (1)

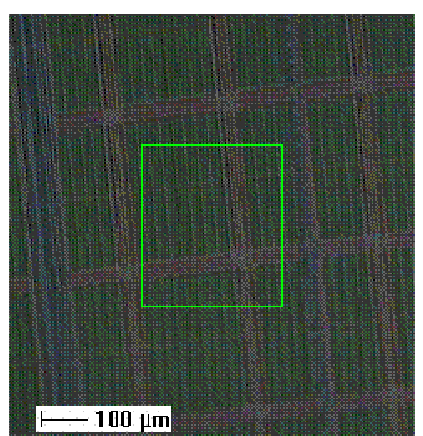

Scribe (2)

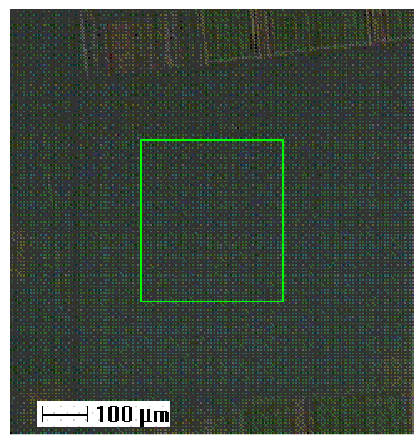

SRAM (3)

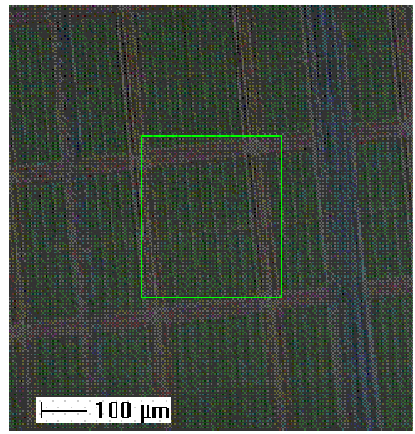

Figure 5.31 Reflectance images on patterned wafers.

\subsection{Summary}

The developed Ti selective wet etch/clean formulation exhibits the ability to remove the plasma etch polymer residue, and strip patterned $\mathrm{Ti}$ thin film layer while suppressing the etch rates of $\mathrm{SiO}_{\mathrm{x}}, \mathrm{CDO}, \mathrm{Si}_{3} \mathrm{~N}_{4}, \mathrm{SiC}, \mathrm{Cu}, \mathrm{W}$. This capability was demonstrated on blanket and DD patterned wafers with no evidence of any adverse impact to interconnect and transistor structures or underlying materials. The results for patterned wafers are in agreement with the blanket wafers etch rates findings for the various substrates.

The formulation synthesis and reaction rate are clearly temperature dependent. Based on the proposed reaction shown in figure 5.10, the break down of MTES at higher temperature and its reaction with $\mathrm{HF}$ under optimal conditions previously described, enhances the formation of the products responsible for the formulation's unique selective characteristics. Since the $\mathrm{pH}$ of the formulation is $\sim 2$, it is expected to rapidly dissolve $\mathrm{Ti}$ as suggested by the Pourbaix diagram shown in Figure 4.4. Although titanium is thermodynamically reactive metal at low $\mathrm{pH}$, titanium is highly resistant to oxidizing acids over a wide range of concentrations and temperatures, 
Hydrofluoric acid and fluoride containing solutions at a wide range of concentrations are capable of attacking titanium. The use of HF containing solutions is problematic since it also dissolves ILD materials such as $\mathrm{SiO}_{\mathrm{x}}, \mathrm{CDO}, \mathrm{SiOF}, \mathrm{Si}_{3} \mathrm{~N}_{4}$. An alternative approach to dissolving titanium is the use of strong oxidizers such as hydrogen peroxide. Unfortunately, the employment of oxidizers readily dissolves tungsten and attack copper.

It has been shown that $\mathrm{WO}_{3}$ is stable under acidic conditions ${ }^{221}$. This oxide layer provides a passivation capability and protects the $\mathrm{W}$ from attack by the chemical solution. Titanium is highly resistant to oxidizing acids due to its unique capacity to form a stable titanium oxide layer $\mathrm{TiO}_{2}^{222}$. A Comparison of thermodynamic stability of $\mathrm{WO}_{3}$ and $\mathrm{TiO}_{2}$ passivating layers given by

$\Delta \mathrm{G}_{\mathrm{f}, 298 \mathrm{~K}}$ for $\mathrm{WO}_{3}=-183 \mathrm{kcal} / \mathrm{mole}$ and $\Delta \mathrm{G}_{\mathrm{f}, 298 \mathrm{~K}}$ for $\mathrm{TiO}_{2}=-213 \mathrm{kcal} / \mathrm{mole}$ shows that $\mathrm{WO}_{3}$ is less thermodynamically stable than $\mathrm{TiO}_{2}$. This indicates that the thermodynamic selectivity of $\mathrm{Ti}$ removal relative to $\mathrm{W}$ in acidic condition is unattainable. Therefore, selectivity may be achievable via kinetic control. Accordingly, the strategy that was applied is to use conditions that are known to protect $\mathrm{W}$. For example, ensuring that the formulation is acidic in nature with $\mathrm{pH}$ equal or below 2 as suggested by the Pourbaix diagram is necessary. Increasing the kinetic rate of Ti dissolution using complexing agents such as EDTA, and/or mild oxidizers were attempted. But these efforts were unsuccessful.

Oxidizers such as $\mathrm{H}_{2} \mathrm{O}_{2}\left(\mathrm{E}_{0}=1.76 \mathrm{~V}\right)$ severely attacked $\mathrm{W}$, while mild oxidizers such as $\mathrm{HCOOH}\left(\mathrm{E}_{0}=0.06 \mathrm{~V}\right), \mathrm{Sn}^{4+}\left(\mathrm{E}_{0}=0.15 \mathrm{~V}\right), \mathrm{Fe}^{3+}$, etc..., were 
fruitless and did not provide the needed wet etch selectivity. Interestingly, the use of dilute $\mathrm{HF}$, one of the strongest oxidizers known $\left(\mathrm{E}_{0}=3.05 \mathrm{~V}\right)$ exhibited compatibility towards W. This compatibility complemented with an increase in selectivity was further enhanced by incorporating MTES into the formulation. Similarly, MTES provided the formulation with its unique compatibility towards $\mathrm{SiO}_{\mathrm{x}}$ and similar materials. The difficulty of this challenge is attributed to the extremely favorable $\mathrm{SiF}_{4}$ $\Delta \mathrm{G}(\approx-1572.7 \mathrm{~kJ} / \mathrm{mole})$ which suggests that $\mathrm{HF}$ reaction with $\mathrm{SiO}_{2}$ is highly spontaneous.

The concept of incorporating a silane cross-linking compound into a dilute HF solution seemed unrealistic. It has been established in the scientific community that silane Cross-linking compounds and other silane molecules form sol-gel polymers in acidic environments. It was hypothesized that silicon precursors may hinder the HF ability to etch silicon oxide and other silicon oxides dielectric materials. This phenomenon can be ascribed to the formation of products such as salicic acid or possibly other species that if present at sufficient amounts in the solution, would establish an equilibrium with $\mathrm{SiO}_{\mathrm{x}}$. Once an equilibrium is reached, silicon oxide and similar materials are no longer etched by HF. Another plausible mechanism is the formation of species that may have passivated the silicon oxide surface.

The excellent electrical performance of the circuit further demonstrated the $\mathrm{Ti}$ etch/clean formulation's capability. A tight distribution obtained in via resistance chain was demonstrated, suggesting that the formulation is suitable for this application. 
The novel feature of this formulation is that although it contains HF, a common agent for etching $\mathrm{SiO}_{\mathrm{x}}$, it does not attack ILD or $\mathrm{SiO}_{2}$ materials. Use of $\mathrm{HF}$ was considered high risk. This formulation represents a first of its kind in meeting demanding requirements in selective metal HM etching in the semiconductor industry and enables the sub $50 \mathrm{~nm}$ and beyond process technologies for ULSI. 


\section{CHAPTER 6}

\section{Formulation Characterization and Reaction Kinetics}

\subsection{Introduction}

Detailed characterization of the various reaction product compounds in the wet etch/clean formulation is presented in this chapter. In this investigation, numerous analytical and characterization techniques were employed in an effort to identify the various reaction products constituents. Here, some reaction products are revealed and carefully characterized by conducting reaction kinetics studies. On the other hand, many product species remain unidentified. This kinetic study was undertaken in an attempt to establish a capability that provides real time reaction/process evaluation and quality control adjustments and fine tuning to ensure successful synthesis. One of the reasons for undertaking this study is for the practical importance of being able to determine how quickly the reaction of HF, MTES, and water approaches equilibrium. Furthermore, the study of the reaction rate leads to an understanding of mechanisms for the investigated reaction and its influence on etch rate of the examined substrates.

Based on the synthesis reaction previously proposed, three product species hexafluorosilicic Acid $\left(\mathrm{H}_{2} \mathrm{SiF}_{6}\right), \mathrm{HF}$, and inorganic silicon compound silica or silicic acid $\left(\mathrm{H}_{2} \mathrm{SiO}_{3}\right)$ were successfully identified. A kinetic investigation was carried out to characterize the behaviors of these species as the reaction progresses. The etch rates

of $\mathrm{Ti}$ and $\mathrm{SiO}_{2}$ as a function of varying $\mathrm{HF}$ concentration resulting during the synthesis was investigated. The reaction orders with respect to $\mathrm{Ti}$ and $\mathrm{SiO}_{2}$ etch rates were 
determined. Furthermore, the mechanisms for the $\mathrm{HF} / \mathrm{SiO}_{2}$ and $\mathrm{HF} / \mathrm{Ti}$ reactions were elucidated. It is important here to evaluate these reactions characteristics due to the uniqueness of the wet etch/clean formulation. It would also be interesting to examine whether the behaviors of these reactions agree with the work of others who studied the etching of $\mathrm{SiO}_{2}$ and dissolution of $\mathrm{Ti}$ in $\mathrm{HF}$ chemistries.

\subsection{Methods}

Various analytical methodologies were employed to aid in the efforts of identifying and characterizing the reaction products. All experiments were done in triplicates. It was readily realized that the determination of the various synthesis products and their structures is extremely challenging. This difficulty stems from the inherent nature of the reaction and its ability to produce many products. The analytical techniques used are listed systematically as follows:

\subsubsection{Analytical Methods}

\section{$F^{-}$Concentration}

The concentration of $\mathrm{F}^{-}$was determined using Orion Model 94-09 Ion Selective Electrode (ISE).

\section{NMR}

Proton NMR spectra were recorded on a $499.89 \mathrm{MHz}$ Varian spectrometer. The NMR spectra were processed using VNMR 4.3 Varian Unity 500 Plus software. ${ }^{19}$ F NMR spectra were recorded on a Bruker AMX600 spectrometer. 


\section{Gas Chromatography Mass Spectrometry (GC-MS)}

The GC-MS system was a Scientific Focus-GC (Thermo-Fisher Scientific). No sample preparation was required for this analysis. The sample was analyzed neat. The samples were hexane extracts of the formulation. One microliter of sample was injected and vaporized in split mode (split ratio 20:1) onto a non-polar GC column where the constituents in the sample were separated under the following temperature conditions: Isothermal at $50 \mathrm{C}$ for 5 minutes, Ramp from 50 to $250{ }^{\circ} \mathrm{C}$ at $10{ }^{\circ} \mathrm{C} / \mathrm{min}$, Hold at $250{ }^{\circ} \mathrm{C}$ for 15 minutes. Under these conditions, compounds with molecular weights up to approximately 400amu can be detected.

Eluted material is swept by helium to a mass spectrometer. The MS acquires data from 40 - 500amu. Spectra are compared to a National Institute of Standards and Technology (NIST) database for identification.

\section{Matrix-Assisted Laser Desorption Mass Spectrometry (MALDI MS)}

MALDI mass spectra were measured on a Bruker Autoflex II reflectron time_of_flight mass spectrometer equipped with a nitrogen laser $(\lambda=337 \mathrm{~nm})$.

\section{High Performance/Pressure Liquid Chromatography- Ultraviolet/Visible Spectroscopy-Mass Spectrometry (HPLC-UV/VIS-MS)}

Dionex High Performance Liquid Chromatography system (UltiMate 3000) was used to carry out the analysis.

\section{Evaporating Light Scattering Detector-Mass Spectrometry (ELSD-MS)}

This work was conducted using the Metara LMS-300 CCA, which is based on in-process mass spectrometry analytical technique. 


\subsubsection{Kinetic Study of Selected Reaction Constituents \\ Determining $\mathrm{H}_{2} \mathrm{SiF}_{6}$ concentration}

\section{Reagents}

De-ionized ice, potassium nitrate-saturated solution, standard Sodium hydroxide solution $(0.05 \mathrm{~N})$, and bromothymol blue (BTB), 0.2 percent solution.

Bromothymol blue is an indicator, a substance that changes color as the $\mathrm{pH}$ of a solution changes. Bromothymol blue is yellow in acidic solutions and blue in basic solutions.

\section{Procedure}

$5 \mathrm{ml}$ of the sample containing the hexafluorosilicic acid was pipetted into a 100 $\mathrm{ml}$ volumetric flask, and then dilute with DI water up to the $100 \mathrm{ml}$ mark. $100 \mathrm{ml}$ of DI ice was placed into a $400 \mathrm{ml}$ beaker. Then $25 \mathrm{ml}$ of potassium nitrate solution was added to the ice. $25 \mathrm{ml}$ of the solution from step 1 was taken and added to the beaker that contains the DI ice and potassium nitrate solution. Three drops of BTB were added to the solution. Titration

with Standard Sodium hydroxide solution $(0.05 \mathrm{~N})$ until the end point is reached was performed (the blue color persists for at least 30 seconds). $\% \mathrm{H}_{2} \mathrm{SiF}_{6}$ can be determined by

$$
\% \mathrm{H}_{2} \mathrm{SiF}_{6}=\frac{N_{\mathrm{NaOH}}(M) V_{\mathrm{NaOH}}(m l) M W_{\mathrm{H}_{2} \mathrm{SiF}_{6}}(g)}{2 . W(g) .1000 m l / L} \times 100
$$

Where $\mathrm{W}$ is the weight of the sample in grams. 


\section{Determining HF concentration}

After cold titration, the beaker was placed with contents from above titration on a hot plate and brought to boil. The hot solution was titrated with sodium hydroxide until the neutral point is reached (green color). $\%$ of free acid (HF) other than $\mathrm{H}_{2} \mathrm{SiF}_{6}$, expressed as

$$
\% H F=\frac{N_{\mathrm{NaOH}}(M)\left(V_{\mathrm{NaOH}}^{\mathrm{cold}}-0.5 V_{\mathrm{NaOH}}^{\text {hot }}\right)(m l) M W_{H F}(g)}{2 . W(g) .1000 m l / L} \times 100
$$

\section{Determining $\mathrm{H}_{2} \mathrm{SiO}_{3}$ Concentration Using Blue Complex Method}

\section{Reagents}

Acidic molybdate solution and reducing agent.

\section{Procedure}

The acidic Molybdate solution is prepared by adding $2 \mathrm{~g}$ of ammonium molybdate tetra hydrate into a $100 \mathrm{ml}$ elementary flask. $6 \mathrm{ml}$ of $37 \%$ HCL were then added. DI water was added up to the $100 \mathrm{ml}$ mark.

The reducing agent is a mix of three different solutions which are prepared as follows:

Solution A: $2 \mathrm{~g}$ of 4-[methyl-amino] phenol sulfate plus $1.2 \mathrm{~g}$ of sodium sulfite anhydrous were placed into a $100 \mathrm{ml}$ elementary flask, then diluted with DI water up to the $100 \mathrm{ml}$ mark.

Solution B: $10 \mathrm{~g}$ of oxalic acid were placed into $100 \mathrm{ml}$ elementary flask, and then diluted up to the $100 \mathrm{ml}$ mark. 
Solution C: $115 \mathrm{~g}$ of $36 \mathrm{~N} \mathrm{H}_{2} \mathrm{SO}_{4}$ were added into $250 \mathrm{ml}$ elementary flask, and then diluted with DI water up to the $250 \mathrm{ml}$ mark to make up $9 \mathrm{~N} \mathrm{H}_{2} \mathrm{SO}_{4}$.

$62.5 \mathrm{ml}$ of the $36 \mathrm{~N} \mathrm{H}_{2} \mathrm{SO}_{4}$ is needed to make up the $250 \mathrm{ml}$ solution of $9 \mathrm{~N}_{2} \mathrm{SO}_{4}$. Multiplying the volume $(62.5 \mathrm{ml})$ by the density of $\mathrm{H}_{2} \mathrm{SO}_{4}(1.84 \mathrm{~g} / \mathrm{ml}), 115 \mathrm{~g}$ of $36 \mathrm{~N}$ $\mathrm{H}_{2} \mathrm{SO}_{4}$ is the amount needed to make up the $9 \mathrm{~N} \mathrm{H}_{2} \mathrm{SO}_{4}$.

The reducing agent is $100 \mathrm{ml}$ of solution A plus $60 \mathrm{ml}$ of solution B plus $120 \mathrm{ml}$ of solution D plus $20 \mathrm{ml}$ of water.

$3 \mathrm{ml}$ of acidic molybdate solution was added to $1 \mathrm{ml}$ of the sample. The solution is heated for 20 minutes at $50-60{ }^{\circ} \mathrm{C}$, until the formation of a yellow complex is noticed. The solution was taken out and $15 \mathrm{ml}$ of the reducing agent was added to it. Reheated for 20 minutes at $50-60{ }^{\circ} \mathrm{C}$. Formation of blue complex is observed. The absorbance was measured at $810 \mathrm{~nm}$ using the UV/Visible (water was ran as blank). A calibration curve is shown in Figure 6.1, was established using silicon standard solutions.

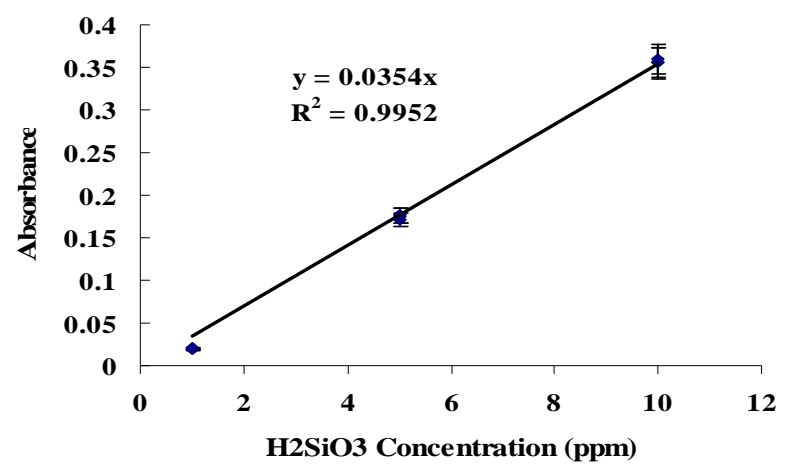

Figure 6.1 Calibration curve constructed using the silicon standard solution. 


\section{Determining Silicon Oxide and Titanium Etch Rates}

$\mathrm{SiO}_{2}$ and $\mathrm{Ti}$ etch rates fro each coupon sample were determined by measuring the thickness before and after immersion in the solution. The thickness measurements for $\mathrm{SiO}_{2}$ were performed using an ellipsometer (Nanometrix, nanospec 5100). The titanium film thickness measurements were performed based on visual observation and utilizing a four point probe method (Surface resistivity meter, Gaurdian Manufacturing Inc., Model SRM-232). All experiments were carried out at $60{ }^{\circ} \mathrm{C}$, using high density poly propylene (HDPP) beaker.

\subsection{Results and Discussion}

\section{$\mathbf{F}^{-}$Concentration}

$\mathrm{F}^{-}$Concentration was identified as a key indictor for synthesis success and completion due to the accuracy and repeatability of measurements. The average concentration of $\mathrm{F}^{-}$was found to be $76.2 \mathrm{ppm}$ after synthesis. The initial goal of this method was to determine and subsequently correlate free fluorine and $\mathrm{SiO}_{2}$ etch rate.

\section{NMR}

Figure 6.2 illustrates ${ }^{1} \mathrm{H}$ NMR of the formulation indicating the presence of ethanol and benzotriazole (Added as a $\mathrm{Cu}$ corrosion inhibitor). It also shows broad multiple peaks in the methyl region (see Figure 6.3). The broad peak could be attributed to fluxional behavior or slow tumbling of oligomers indicating the presence of methyl group in oligomeric species. 


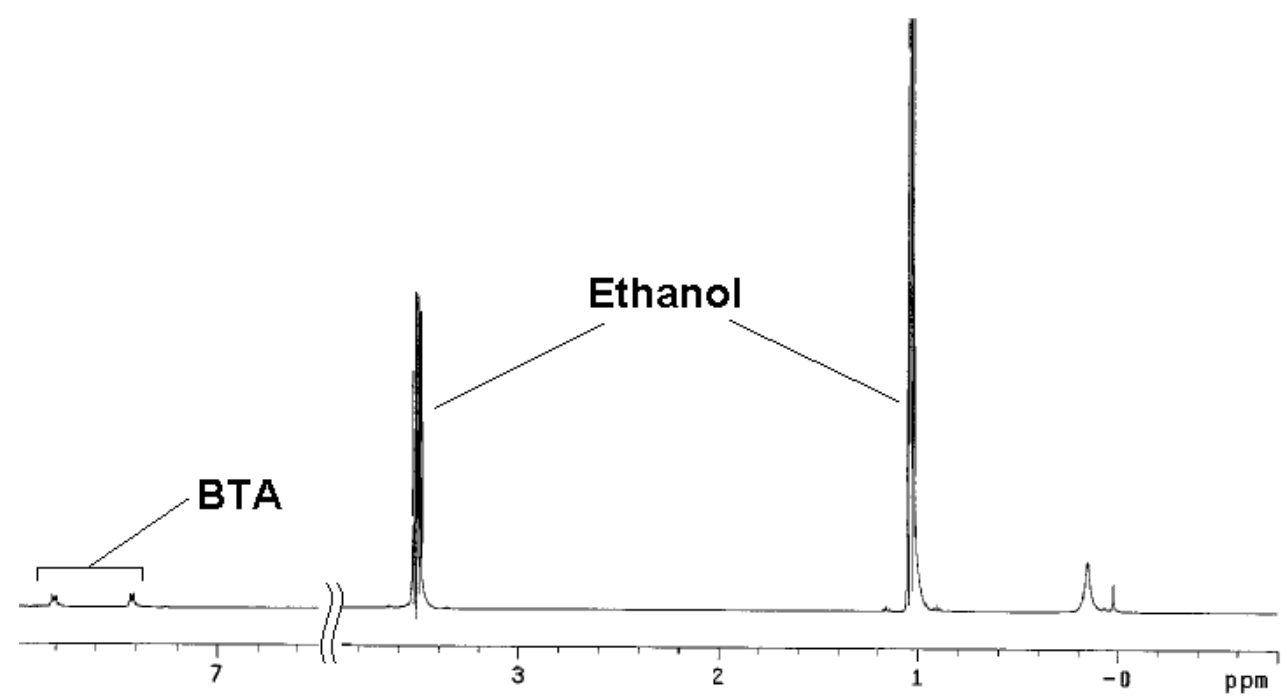

Figure 6.2 ${ }^{1} \mathrm{H}$ NMR spectrum of wet etch/clean formulation.

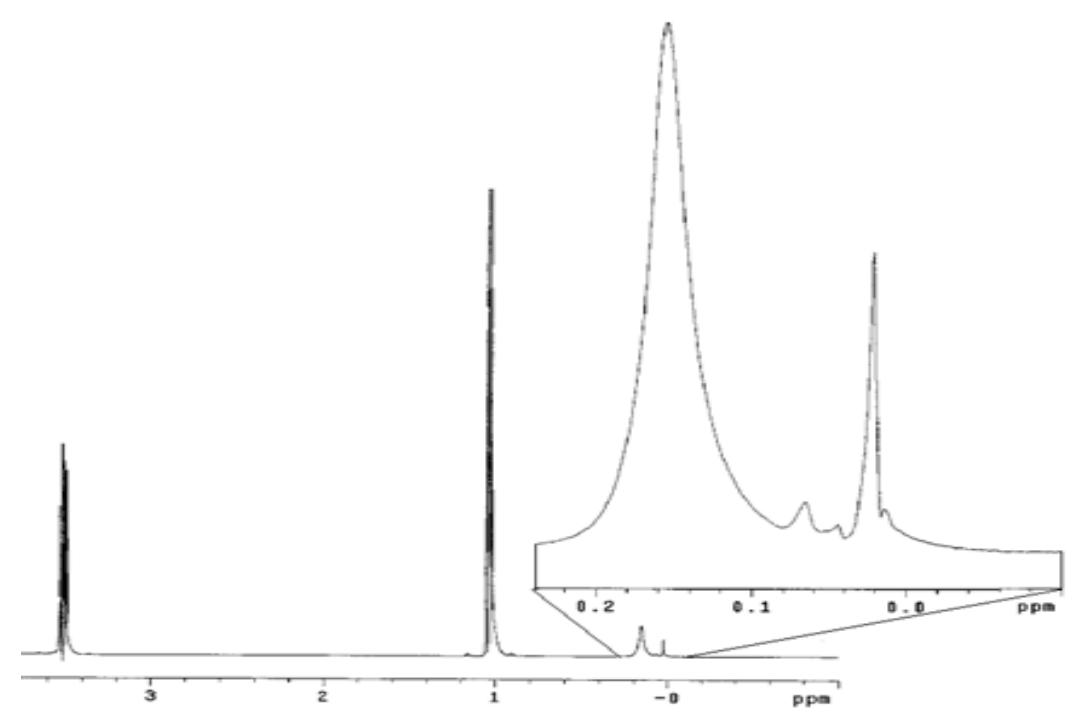

Figure 6.3 ${ }^{1} \mathrm{H}$ NMR spectrum. Note multiple peaks in the methyl region.

${ }^{19} \mathrm{~F}$ NMR shows similar broad peaks as illustrated in Figure 6.4, which could also be attributed to fluxional behavior or overlapping peaks due to oligomers. ${ }^{19} \mathrm{~F}$ NMR of dilute HF solution gave two peaks at $129 \mathrm{ppm}$ (possibly $\mathrm{H}_{2} \mathrm{SiF}_{6}$ ), and a peak at $158 \mathrm{ppm}$ (due to HF) as shown in Figure 6.5. 


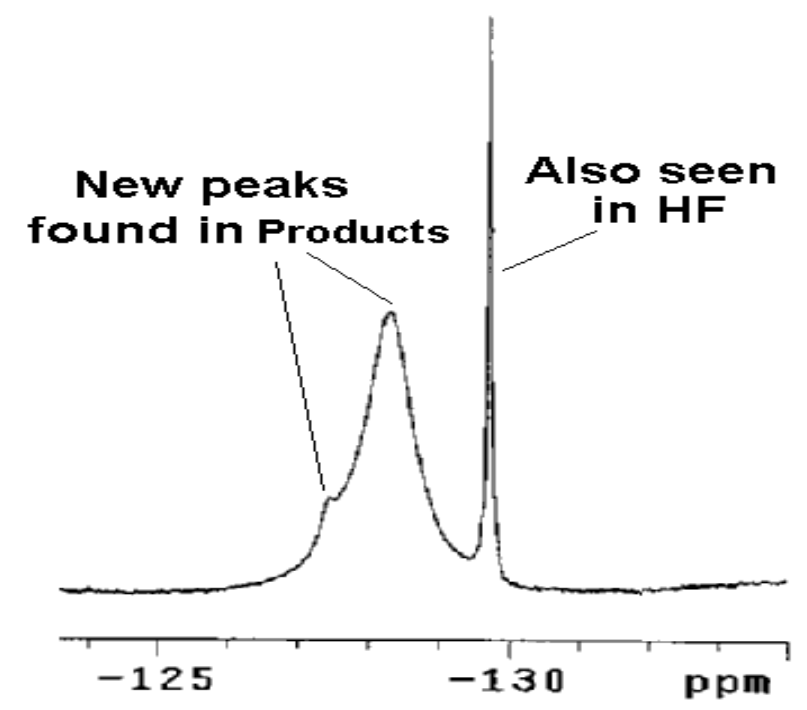

Figure 6.4 ${ }^{19}$ F NMR spectrum of formulation.

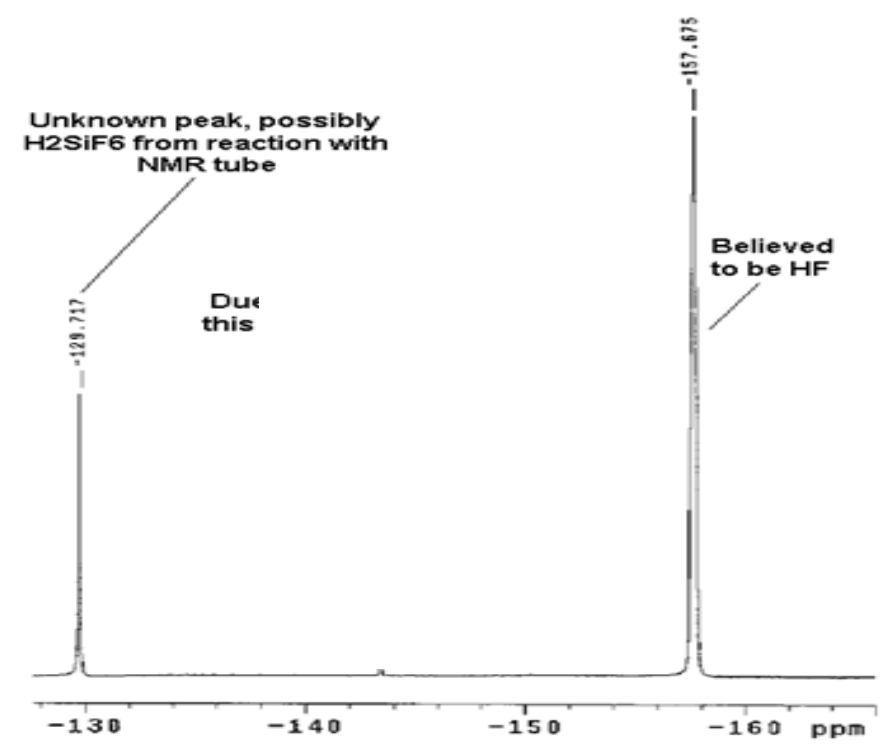

Figure 6.5 ${ }^{19} \mathrm{~F}$ NMR spectrum of pure HF.

${ }^{19}$ F NMR illustrated in Figure 6.6 shows the absence of the peak at $158 \mathrm{ppm}$ seen in HF spectrum (Figure 6.5). Absence of this peak could suggest that free HF is reacted out of the solution forming $\mathrm{SiF}$ oligomeric species leading to a broad peak at 
127-129 ppm as shown in Figure 6.4. The entire ${ }^{19} \mathrm{~F}$ NMR spectrum of the formulation is shown in Figure 6.7 for reference.

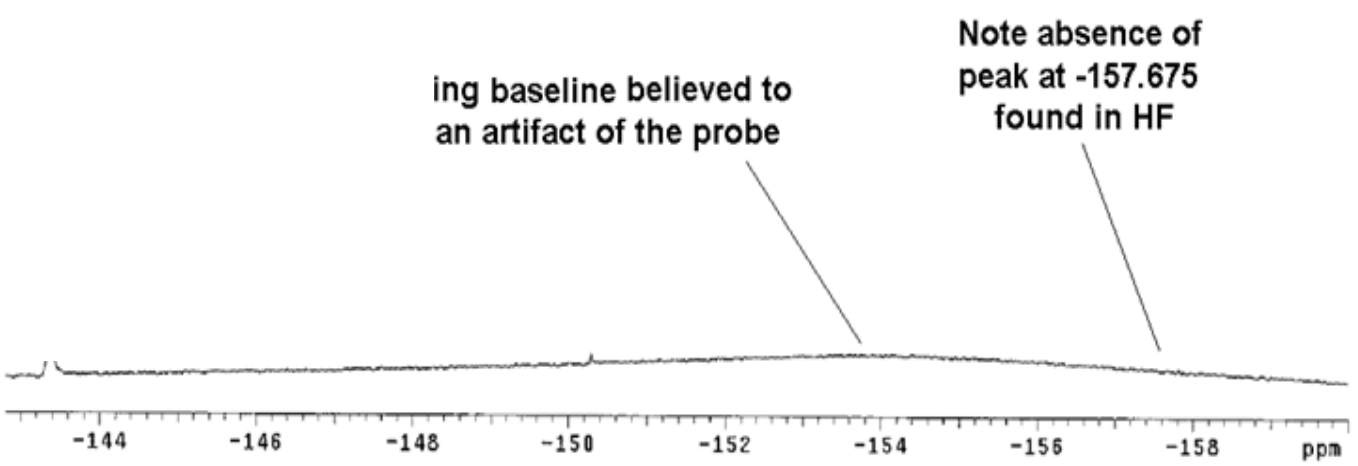

Figure 6.6 ${ }^{19} \mathrm{~F}$ NMR spectrum of formulation. Note absence of peak at -157.7 .

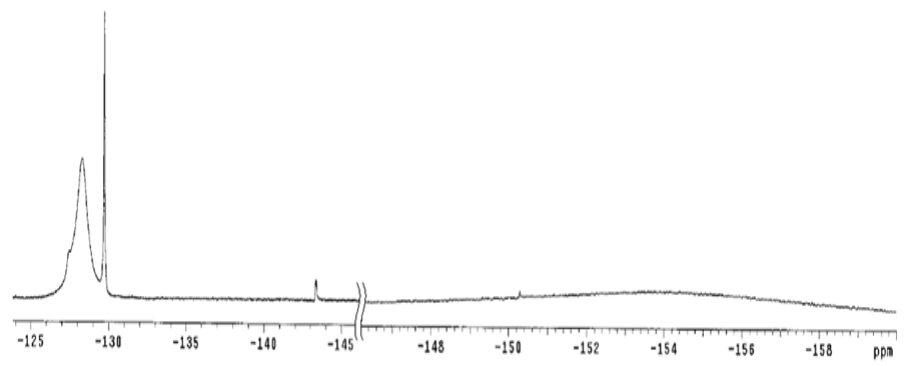

Figure 6.7 Entire ${ }^{19}$ F NMR spectrum of formulation is shown for reference.

\section{Gas Chromatography Mass Spectrometry (GC-MS)}

The goal of this technique was to achieve mixture separation and discrete molecular detection. GC-MS data shown in figure 6.8 indicate a grouping of peaks that are separated by 77 mass units, a fragment consistent with the polymer backbone $\mathrm{CH}_{3} \mathrm{Si}(\mathrm{OH})_{2}$ or $\mathrm{CH}_{2} \mathrm{SiOF}^{-}$. Each oligomer then cleaves in a predictable pattern Groupings of peaks are believed to be analogs having varying numbers OEt or $\mathrm{F}$ ligands. 


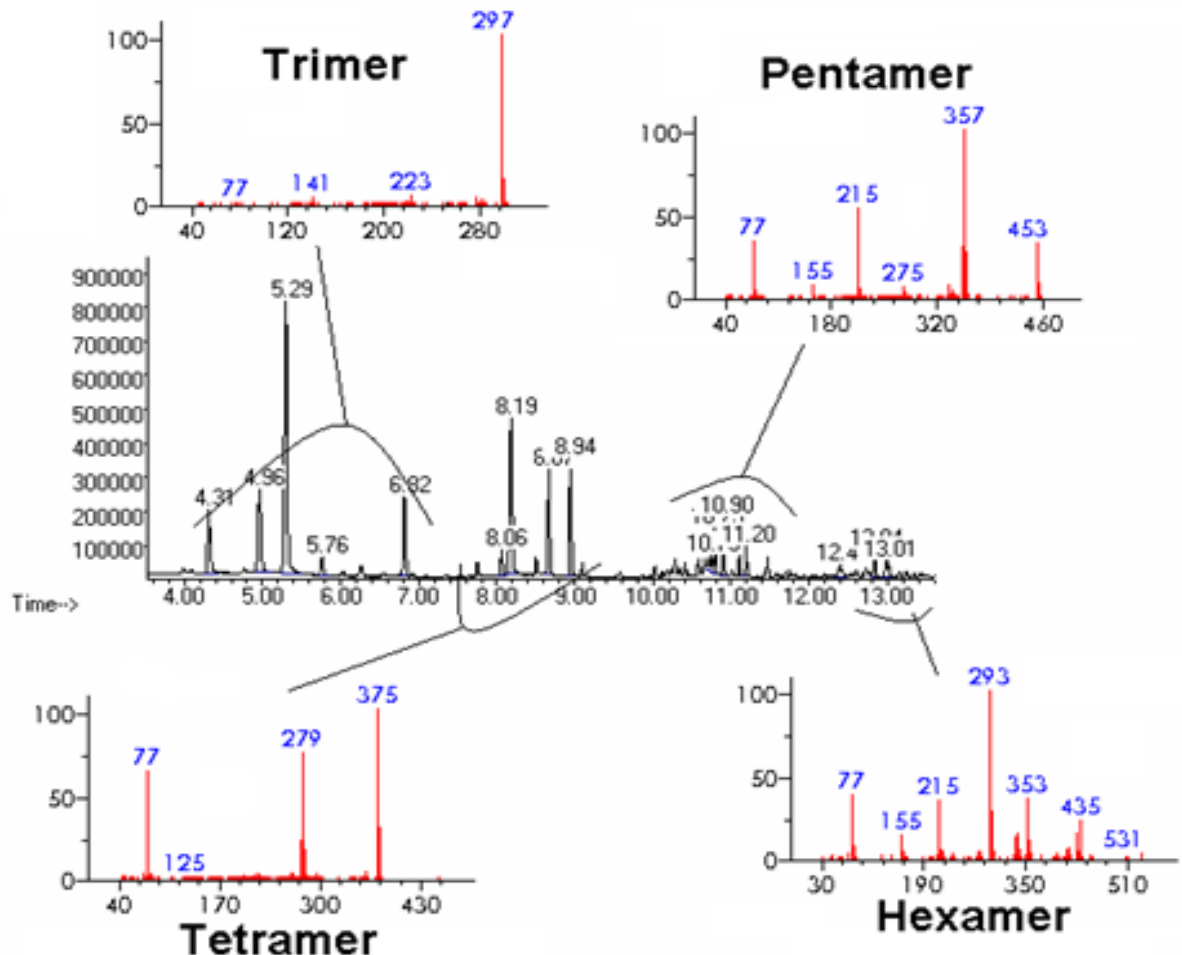

Figure 6.8 GC-MS Spectrum indicates grouping of peaks that are separated by 77 mass units.

\section{Matrix-Assisted Laser Desorption Mass Spectrometry (MALDI MS)}

The purpose of this analysis was to determine molecular ions and fragments in the formulation. MALDI work results illustrated in Figure 6.9 shows similar, predictable cleaving patterns when compared to GC-MS results. The results indicate fragmentation pattern of $14\left(\mathrm{CH}_{2}\right)$ and $58\left(\mathrm{CH}_{2} \mathrm{SiO}\right)$ mass units. However, the predictable fragmentation pattern ends at mass units below 169 as depicted in Figure 6.10. Lower mass spectra consist of several prominent mass peaks other than identified repeating fragments. This may indicate the presence of monomer fragments. 


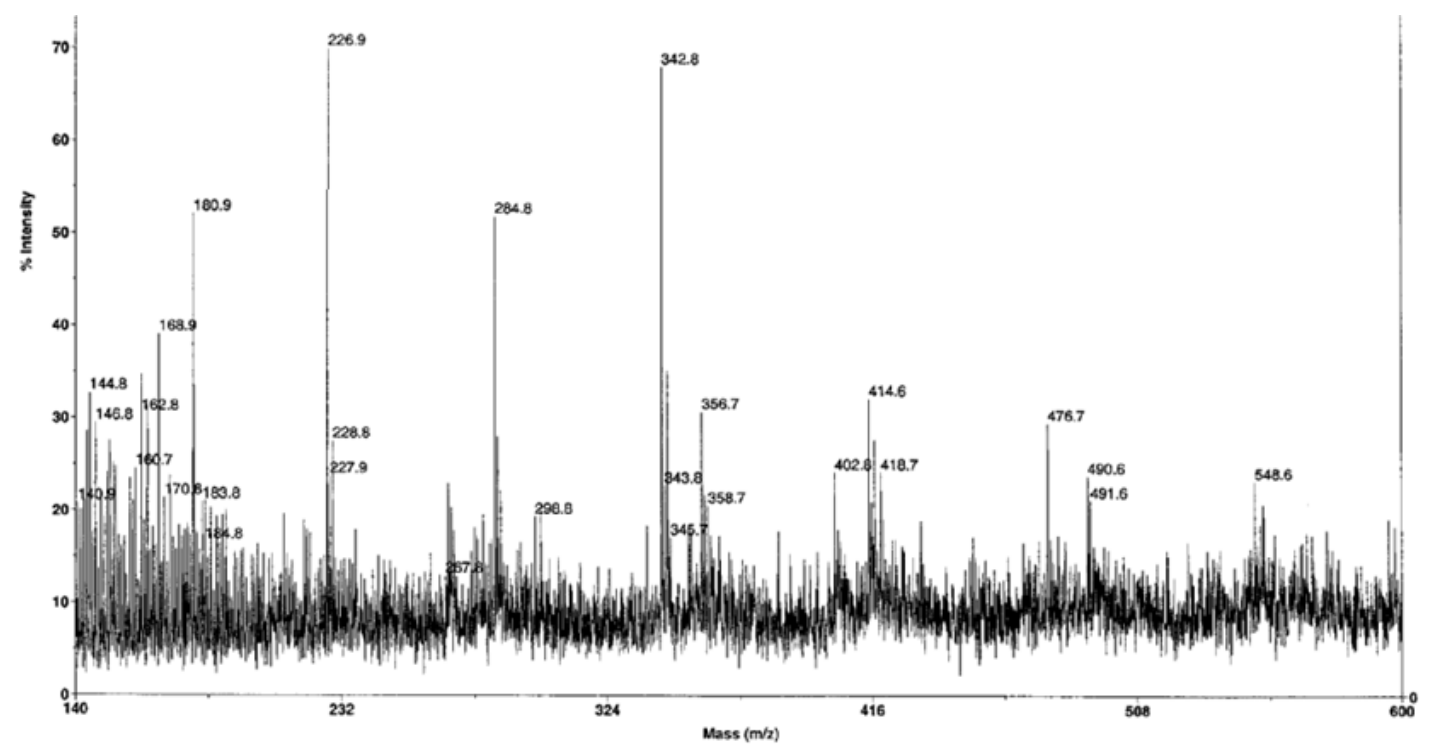

Figure 6.9 MALDI-MS spectrum for the formulation.

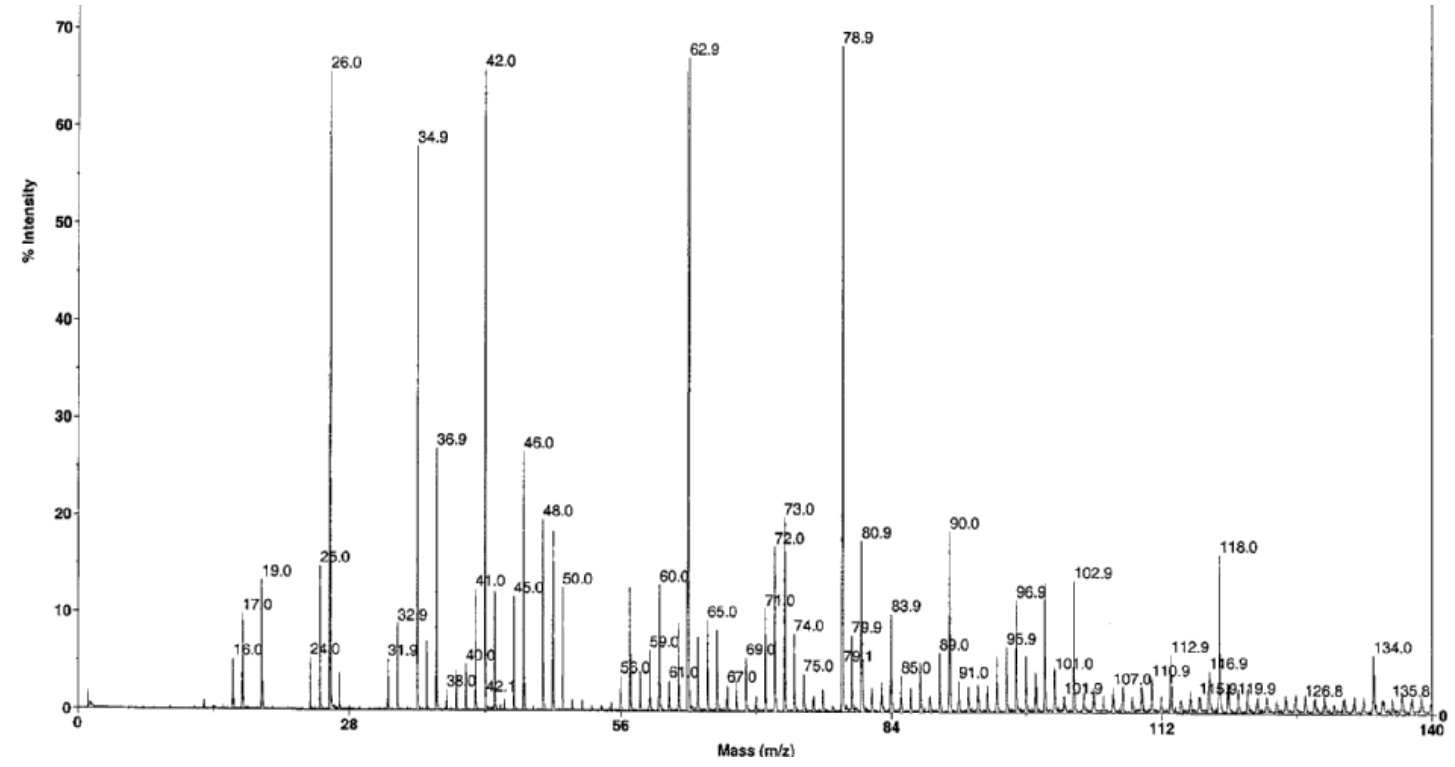

Figure 6.10 MALDI lower mass spectrum of the formulation. Note fragmentation pattern ends at mass units below 169. 


\section{High Performance/Pressure Liquid Chromatography-Ultraviolet/Visible Spectroscopy-Mass Spectrometry (HPLC-UV/VIS-MS)}

HPLC technique was employed for species separation. The identification of compounds and interpretation of their structure was done by using UV/VIS and MS respectively. Figure 6.11 shows the results of this study in which the presence of BTA is confirmed. Other species were not detected in this region. This emerged as an excellent technique for BTA analysis but lacks the selectivity for separating out formulation components. This was a practical quantitative analytical technique for BTA while also having the ability to monitor for BTA degradation.

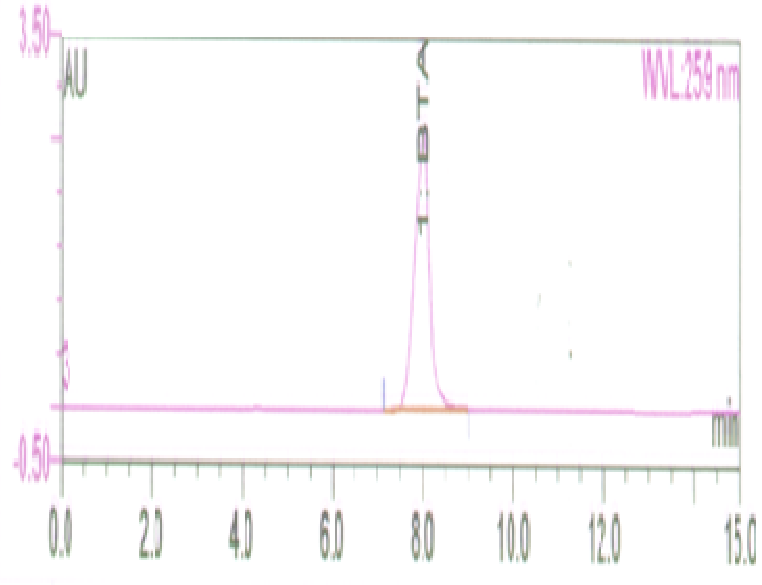

(a)

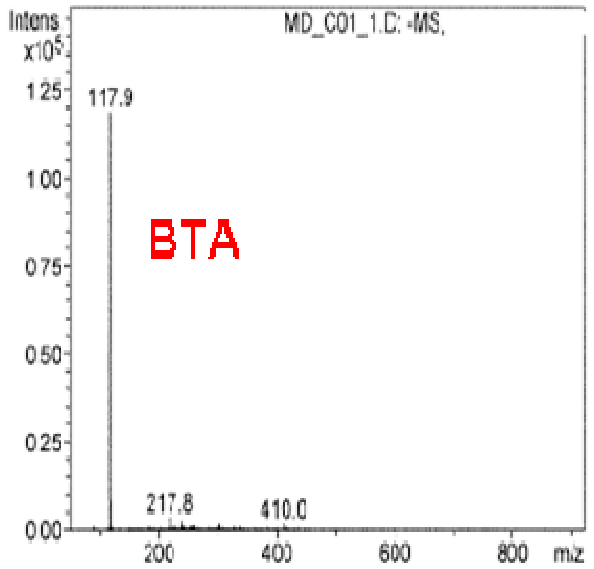

(b)

Figure 6.11 UV/VIS and MS spectrums in (a) and (b) respectively, indicating the presence of BTA in the UV/VIS region.

\section{Evaporating Light Scattering Detector-Mass Spectrometry (ELSD-MS)}

The ELSD-MS technique was used to complement other analytical methods in identifying the various oligomer/polymer species in the formulation. The result of this study is shown in Figure 6.12 a and b for the ELSD and MS respectively. 


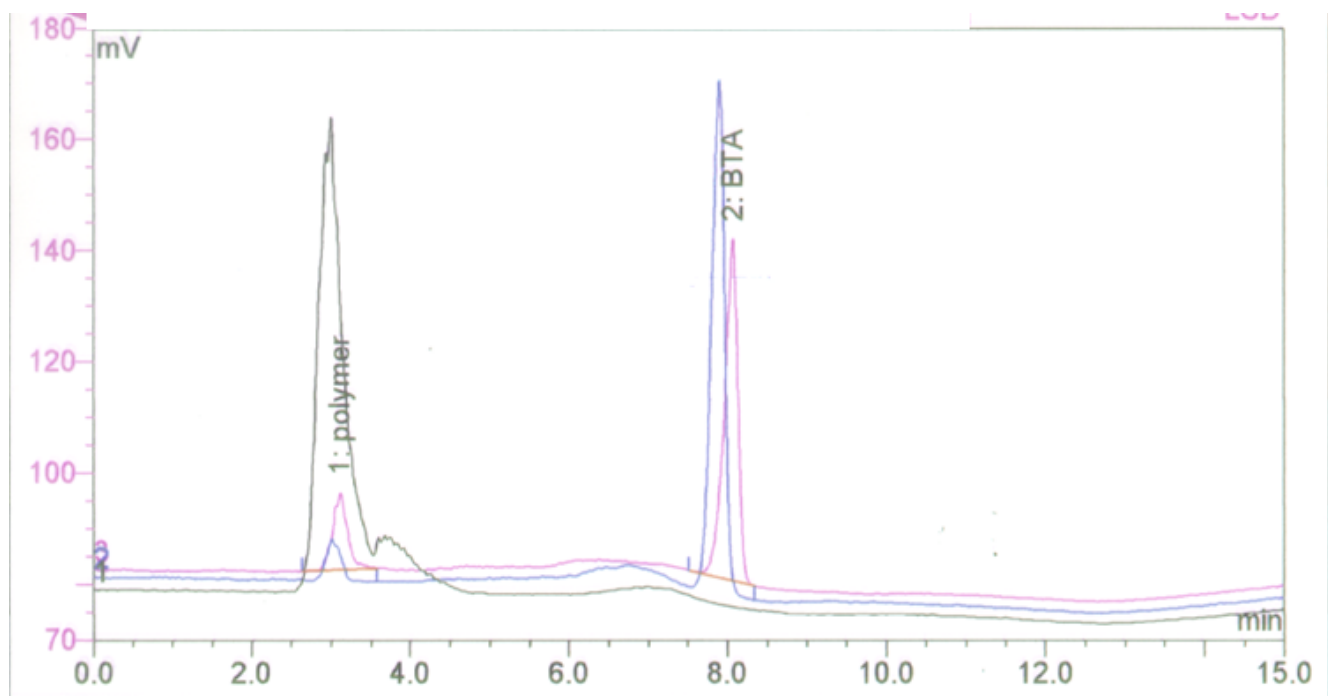

(a)

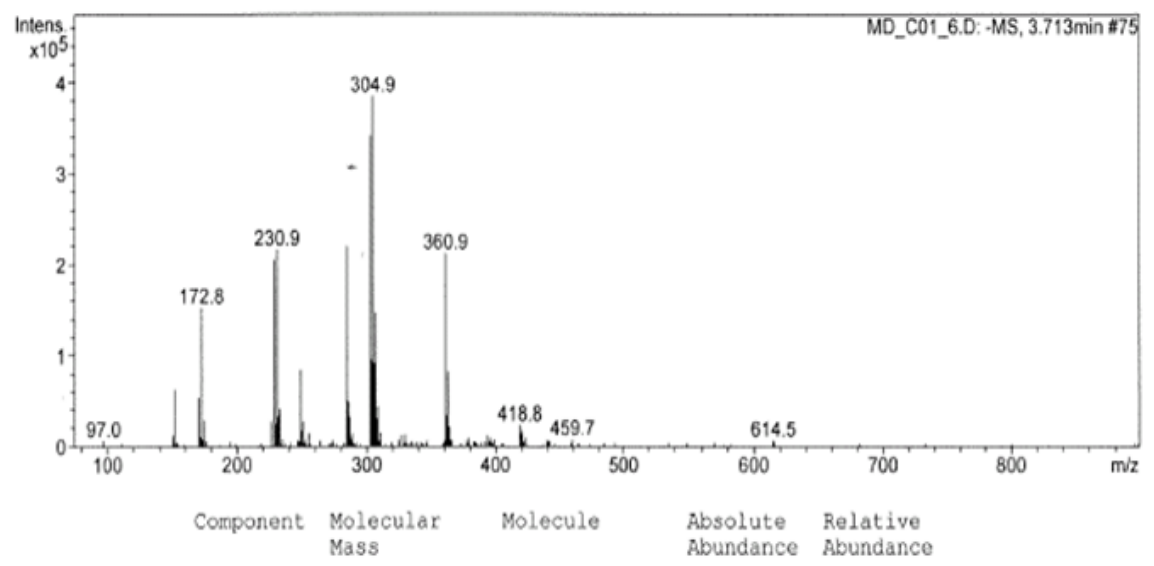

(b)

Figure 6.12 ELSD in (a) and MS spectrums in (b) confirming the presence of BTA and other species.

\subsection{Kinetic Modeling of Formulation Synthesis}

Kinetic model and reactions mechanisms were developed for the HF/MTES/water reaction. This kinetic work is relevant for establishing a successful synthesis and, more importantly, serves as a model to fine tuning the reaction behaviors during the synthesis. One of the reasons for undertaking this study is for the 
practical importance of being able to determine how quickly the reaction of HF, MTES, and water approaches equilibrium. Furthermore, the study of the reaction rate leads to an understanding of mechanisms for the investigated reaction and its influence on etch rate of the examined substrates.

Based on the synthesis reaction previously proposed, three product species hexafluorosilicic Acid $\left(\mathrm{H}_{2} \mathrm{SiF}_{6}\right), \mathrm{HF}$, and an inorganic silicon compound (silicic acid) were successfully identified. A kinetic investigation was carried out to characterize the behaviors of these species as the reaction progresses. The etch rates of $\mathrm{Ti}$ and $\mathrm{SiO}_{2}$ as a function of varying $\mathrm{HF}$ concentration resulting during the synthesis was investigated. The reaction orders with respect to $\mathrm{Ti}$ and $\mathrm{SiO}_{2}$ etch rates was determined. Furthermore, the mechanisms for the $\mathrm{HF} / \mathrm{SiO}_{2}$ and $\mathrm{HF} / \mathrm{Ti}$ reactions were elucidated. Although these reactions have been extensively studied, it is important here to evaluate their characteristics due to the uniqueness of the wet etch/clean formulation. It would also be interesting to examine whether the behaviors of these reactions agree with the work of others ${ }^{223}$ who studied the etching of $\mathrm{SiO}_{2}$ and dissolution of Ti in HF chemistries.

\subsubsection{Kinetic Study Results}

The experimental results pertaining to the synthesis of the formulation to evaluate silicic acid $\left(\mathrm{H}_{2} \mathrm{SiO}_{3}\right), \mathrm{H}_{2} \mathrm{SiF}_{6}$, and $\mathrm{HF}$ concentrations, and $\mathrm{SiO}_{2}$ and Ti etch rates are illustrated in Table 6.1. Etch rates of $\mathrm{Ti}$ and $\mathrm{SiO}_{2}$ and concentrations of reaction mixture were monitored as a function of synthesis time. The study was carried out over a 24 hours period in which HF (0.24 wt $\%)$, MTES (1.66 wt $\%)$, and 
water (98 wt\%) were mixed. The data indicates a strong correlation between HF concentration and $\mathrm{Ti}$ and $\mathrm{SiO}_{2}$ etch rates. On the other hand, the correlation of $\mathrm{H}_{2} \mathrm{SiO}_{3}$ and $\mathrm{H}_{2} \mathrm{SiF}_{6}$ is not as robust. Furthermore, the data shows that the reaction/synthesis reaches equilibrium in less than two hours. This is apparent when comparing the concentration and etch rate results for the 2 hour and 24 hours samples.

Table 6.1 $\mathrm{H}_{2} \mathrm{SiO}_{3}, \mathrm{H}_{2} \mathrm{SiF}_{6}$, and $\mathrm{HF}$ concentrations, and Etch rates of $\mathrm{SiO}_{2}$ and $\mathrm{Ti}$ determined in real time while synthesis is in progress.

\begin{tabular}{|c|c|c|c|c|c|c|c|c|c|}
\hline $\begin{array}{l}\text { Time } \\
(\min )\end{array}$ & $\begin{array}{c}\text { H2SiO3 } \\
\text { Concentration }(\mathrm{ppm})\end{array}$ & $\begin{array}{l}\text { H2SiF6 } \\
(w t \%)\end{array}$ & $\begin{array}{c}\mathrm{HF} \\
(\mathrm{wt} \%)\end{array}$ & $\begin{array}{c}\mathrm{SiO} 2 \mathrm{ER} \\
(\mathrm{A} / \mathrm{min})\end{array}$ & $\begin{array}{c}\text { Ti ER } \\
(\mathbf{A} / \mathrm{min})\end{array}$ & $\operatorname{Ln}[\mathrm{HF}]$ & $\begin{array}{c}\mathrm{Ln}(\mathrm{SiO} 2 \\
\mathrm{ER})\end{array}$ & $\begin{array}{l}\text { Ln }(\mathbf{T i} \\
\mathbf{E R})\end{array}$ & $\begin{array}{l}\text { Selectivity (Ti } \\
\text { ER/SiO2 ER) }\end{array}$ \\
\hline $\begin{array}{c}0.0 \\
\end{array}$ & $\begin{array}{l}3.500 \\
\end{array}$ & $\begin{array}{l}0.008 \\
\end{array}$ & $\begin{array}{l}0.172 \\
\end{array}$ & $\begin{array}{l}3.70 \\
\end{array}$ & 164.84 & $\begin{array}{l}-1.760 \\
\end{array}$ & $\overline{1.308}$ & $\overline{\mid 5.105}$ & 44.550 \\
\hline$\overline{30.0}$ & 5.100 & 0.005 & $\overline{0.083}$ & 1.00 & 96.15 & -2.489 & $\overline{0.000}$ & 4.566 & 96.154 \\
\hline 60.0 & 4.300 & 0.005 & 0.059 & 0.73 & 94.94 & -2.830 & $\begin{array}{l}-0.315 \\
\end{array}$ & 4.553 & 130.050 \\
\hline 90 & 4.500 & 0.004 & $\begin{array}{l}0.043 \\
\end{array}$ & 0.37 & 47.62 & $\begin{array}{l}-3.147 \\
\end{array}$ & $\begin{array}{c}-0.994 \\
\end{array}$ & 3.863 & 128.700 \\
\hline 120.0 & 4.800 & 0.004 & 0.037 & 0.25 & 45.45 & -3.297 & -1.386 & 3.817 & 181.818 \\
\hline 1440.0 & 4.700 & 0.004 & 0.037 & 0.26 & 45.30 & -3.297 & -1.347 & 3.813 & 174.231 \\
\hline
\end{tabular}

During the synthesis of formulation, hydrolysis of Mtes may be represented schematically as:

$$
\begin{aligned}
& \text { Mtes }+3 \mathrm{H}_{2} \mathrm{O} \rightarrow \mathrm{Si}(\mathrm{OH})_{4}+3 \mathrm{ROH} \\
& \mathrm{Si}(\mathrm{OH})_{4}+6 \mathrm{HF} \leftrightarrow \mathrm{H}_{2} \mathrm{SiF}_{6}+4 \mathrm{H}_{2} \mathrm{O} \\
& \mathrm{K}=\frac{\left[\mathrm{H}_{2} \mathrm{SiF}_{6}\right]}{\left[\mathrm{Si}(\mathrm{OH})_{4}\right][\mathrm{HF}]^{6}} \approx \frac{2.8 \times 10^{-5}}{6.4 \times 10^{-5} \times\left[1.9 \times 10^{-2}\right]^{6}} \approx 1 \times 10^{10}
\end{aligned}
$$

The $\mathrm{K}$ value estimated from the concentrations in Table 6.1 is very large implying a complete disappearance of HF consistent with the NMR results shown in Figures 6.46.7. The figures show ${ }^{19} \mathrm{~F}$ NMR peak due to HF completely disappears upon completion of the synthesis. Furthermore, this result is consistent with studies of Finney et. al. ${ }^{224}$ who found similarly a large value for $\mathrm{K}$ in their study of $\mathrm{H}_{2} \mathrm{SiF}_{6}$ 
hydrolysis. It is known that under acidic conditions $(\mathrm{pH}<4)$ the polymerization of $\mathrm{Si}(\mathrm{OH})_{\mathrm{x}}$ can be prevented, although experimental data [NMR and Mass spectroscopy, see figure 6.8-6.10] indicate some degree of oligomerization during the course of the synthesis. More importantly, the extremely low concentration of HF detected provides protection against unwanted etching of $\mathrm{SiO}_{\mathrm{x}}$ structures on wafer during $\mathrm{Ti}$ etching process.

To further probe the effect of $\mathrm{HF}$ and predict the reactions order kinetics for $\mathrm{Ti}$ dissolution and $\mathrm{SiO}_{2}$ etch rate, the $\log$ of $\mathrm{HF}$ concentration vs. the $\log$ of $\mathrm{SiO}_{2}$ and $\mathrm{Ti}$ etch rates were investigated as shown in Figures 6.13, and 6.15 respectively. Furthermore, the selectivity of $\mathrm{Ti}$ to $\mathrm{SiO}_{2}\left(\mathrm{Ti} \mathrm{ER} / \mathrm{SiO}_{2} \mathrm{ER}\right)$ was examined as shown in figure 6.16.

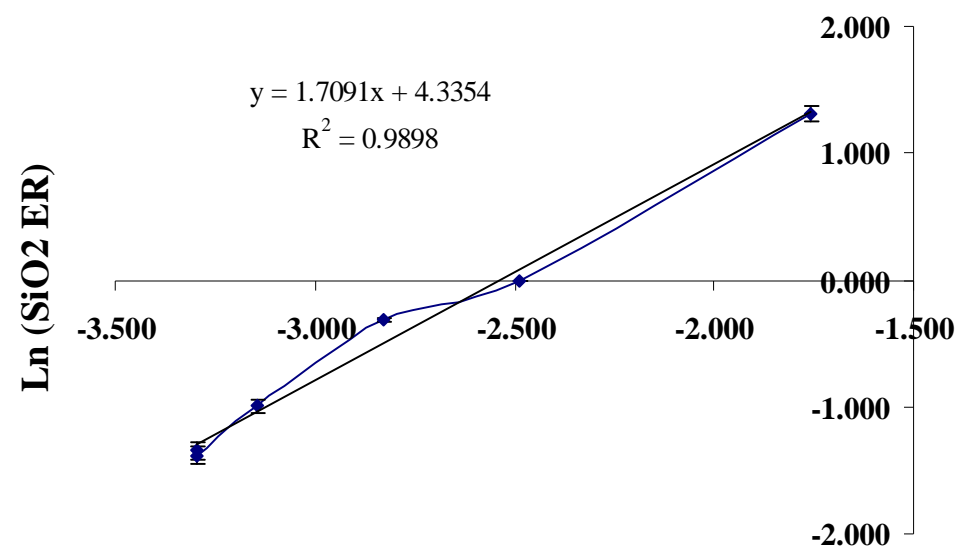

$\operatorname{Ln}[\mathbf{H F}]$

Figure 6.13 $\mathrm{SiO}_{2}$ ER Kinetic Order with Respect to HF Concentration.

The observed $\mathrm{SiO}_{2}$ etch rate order with respect to $\mathrm{HF}$ concentration is 1.7. This order value is consistent with the results of Monk et. al. ${ }^{225}$ who observed similar order for phosphorus doped silicon oxide. It is believed that oxide etching results 
from attack of $\mathrm{HF}_{2}{ }^{-}$. As previously discussed during the synthesis, $\mathrm{HF}$ concentration decreases dramatically as depicted in Figure 6.14.

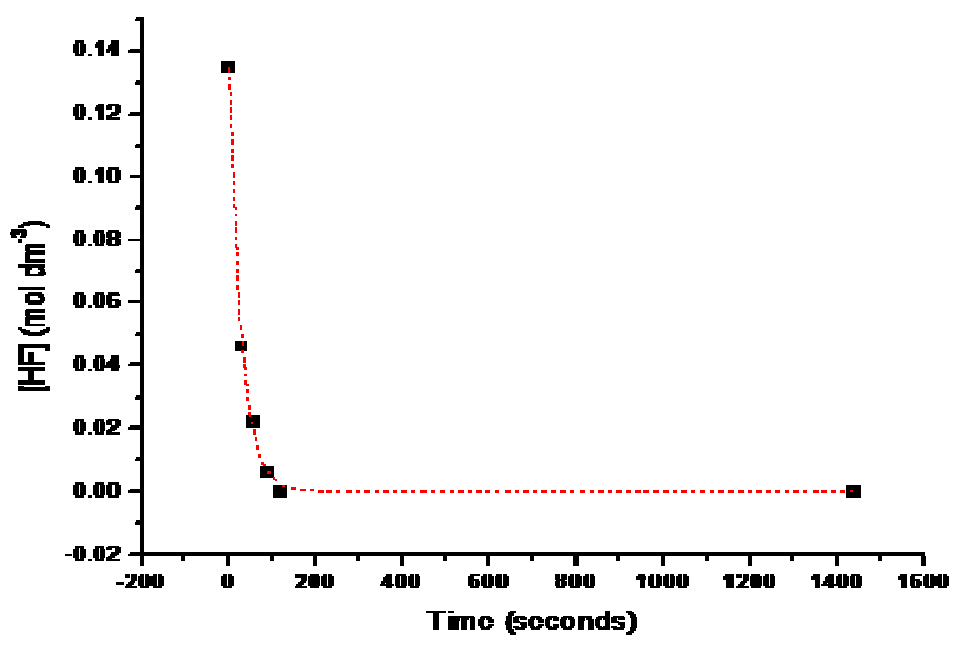

Figure 6.14 HF concentration as a function of synthesis time shows exponential decay, with time constant of 29 minutes.

This exponential time dependence of HF concentration during the synthesis can be explained as follows: 


$$
\begin{aligned}
& \mathrm{CH}_{3} \mathrm{Si}(\mathrm{OEt})_{3}+3 \mathrm{H}_{2} \mathrm{O} \stackrel{\mathrm{H}^{+}}{\longrightarrow} \mathrm{CH}_{3} \mathrm{Si}(\mathrm{OH})_{3}+3 \mathrm{C}_{2} \mathrm{H}_{5} \mathrm{OH} \\
& \mathrm{CH}_{3} \mathrm{Si}(\mathrm{OH})_{3}+3 \mathrm{HF}_{k_{-1}}^{\mathrm{K}_{1}} \mathrm{CH}_{3} \mathrm{SiF}_{3}+3 \mathrm{H}_{2} \mathrm{O} \\
& -\frac{1}{3} \frac{d[\mathrm{HF}]}{d t}=k_{1}[\mathrm{HF}]^{3}\left[\mathrm{CH}_{3} \mathrm{Si}(\mathrm{OH})_{3}\right]-k_{-1}\left[\mathrm{CH}_{3} \mathrm{SiF}_{3}\right] \\
& {[\mathrm{HF}]=[\mathrm{HF}]_{E q}+\Delta[\mathrm{HF}]} \\
& {\left[\mathrm{CH}_{3} \mathrm{Si}(\mathrm{OH})_{3}\right]=\left[\mathrm{CH}_{3} \mathrm{Si}(\mathrm{OH})_{3}\right]_{E q}+\Delta\left[\mathrm{CH}_{3} \mathrm{Si}(\mathrm{OH})_{3}\right]} \\
& =\left[\mathrm{CH}_{3} \mathrm{Si}(\mathrm{OH})_{3}\right]_{E q}+\frac{\Delta[\mathrm{HF}]}{3} \\
& {\left[\mathrm{CH}_{3} \mathrm{SiF}_{3}\right]=\left[\mathrm{CH}_{3} \mathrm{SiF}_{3}\right]_{E q}+\Delta\left[\mathrm{CH}_{3} \mathrm{SiF}_{3}\right]} \\
& =\left[\mathrm{CH}_{3} \mathrm{SiF}\right]_{E_{E q}}-\frac{\Delta[\mathrm{HF}]}{3} \\
& \therefore-\frac{1}{3} \frac{d[\Delta H F]}{d t}=k_{1}\left[[\mathrm{HF}]_{e q}+\Delta[\mathrm{HF}]\right]^{3}\left[\left[\mathrm{CH}_{3} \mathrm{Si}(\mathrm{OH})_{3}\right]_{E q}+\frac{\Delta[\mathrm{HF}]}{3}\right] \\
& \left.\quad-k_{-1}\left[\mathrm{CH}_{3} \mathrm{SiF}\right]_{E q}-\frac{\Delta[\mathrm{HF}]}{3}\right]
\end{aligned}
$$

Keeping terms first order in $\Delta[H F]$

$$
\begin{aligned}
&-\frac{1}{3} \frac{d[\Delta H F]}{d t}=k_{1}\left[[H F]_{E q}^{3}+3 \Delta[H F][H F]_{E q}^{2}\right]\left[\left[\mathrm{CH}_{3} \mathrm{Si}(\mathrm{OH})_{3}\right]_{E q}+\frac{\Delta[H F]}{3}\right] \\
&-k_{-1}\left[\left[\mathrm{CH}_{3} \mathrm{SiF}_{3}\right]_{E q}-\frac{\Delta[H F]}{3}\right] \\
&-\frac{1}{3} \frac{d[\Delta H F]}{d t}= k_{1}\left[[H F]_{E q}^{3} \frac{\Delta[H F]}{3}+3 \Delta[H F]\left[\mathrm{CH}_{3} \mathrm{Si}(\mathrm{OH})_{3}\right]_{E q}[H F]_{E q}^{2}\right] \\
&+k_{-1}\left[\frac{\Delta[H F]}{3}\right]
\end{aligned}
$$

Recalling

$$
\begin{aligned}
& \mathrm{K}_{e q}=\frac{k_{1}}{k_{-1}}=\frac{\left[\mathrm{CH}_{3} \mathrm{SiF}_{3}\right]_{E q}}{[\mathrm{HF}]_{E q}^{3}\left[\mathrm{CH}_{3} \mathrm{Si}(\mathrm{OH})_{3}\right]_{E q}} \\
& \frac{d[\Delta H F]}{d t}=-\left[k_{1}\left[[\mathrm{HF}]_{E q}^{3}+9\left[\mathrm{CH}_{3} \mathrm{Si}(\mathrm{OH})_{3}\right]_{E q}[\mathrm{HF}]_{E q}^{2}+k_{-1}\right][\Delta H F]\right. \\
& \Delta H F=\Delta H F^{0} e^{-\alpha t} \quad \text { with } \alpha=\left[k_{1}\left[[\mathrm{HF}]_{E q}^{3}+9\left[\mathrm{CH}_{3} \mathrm{Si}(\mathrm{OH})_{3}\right]_{E q}[\mathrm{HF}]_{E q}^{2}+k_{-1}\right]\right.
\end{aligned}
$$

As the equilibrium concentration of $\mathrm{HF}$ is low, the $\mathrm{HF}_{2}{ }^{-}$concentration would be even lower thereby preventing oxide etching. 
Similar analysis of $\mathrm{Ti}$ etch rate with respect to $\mathrm{HF}$ concentration gave a reaction order of approximately one as shown in Figure 6.15.

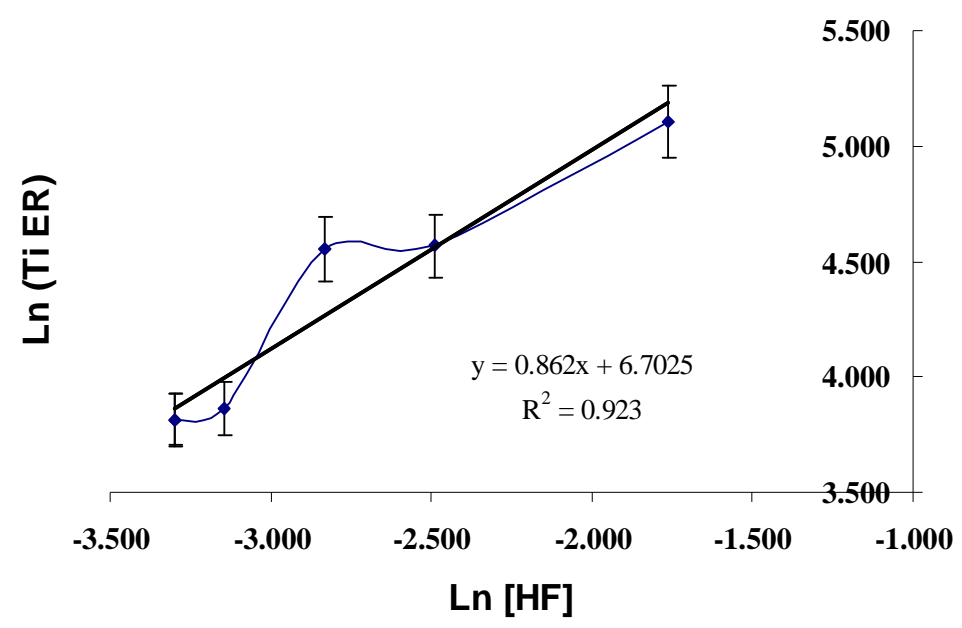

Figure 6.15 Ti ER Kinetic Order with Respect to HF Concentration.

This kinetic order is similar to what observed for copper etching by HF in chapter 3. By analogy with copper etching studies, it is suggested that Ti etching may proceed through formation of titanium oxide on the surface. No further surface characterization of Ti species or corrosion studies were conducted.

From application point of view, what is important is the selectivity of $\mathrm{Ti}$ etching over $\mathrm{SiO}_{2}$ etching. The selectivity as defined by equation 4-2 is plotted as a function of HF concentration in Figure 6.16. It clearly shows an exponential increase in selectivity with decrease in HF concentration. The Pourbaix diagram for Ti suggests two scenarios depending on the open circuit potential (OPC). If OPC is close to zero, the formation of $\mathrm{TiO}_{2}$, which in turn is dissolved by $\mathrm{HF}$, could provide the $\mathrm{Ti}$ etching mechanism. On the other hand if the OPC is significantly negative, then direct attack of fluoride on Ti could drive the dissolution process (see below). If the former 
were the mechanism, etching rate will be sensitive to dissolved oxygen. Further studies are necessary to establish molecular mechanism for Ti dissolution.

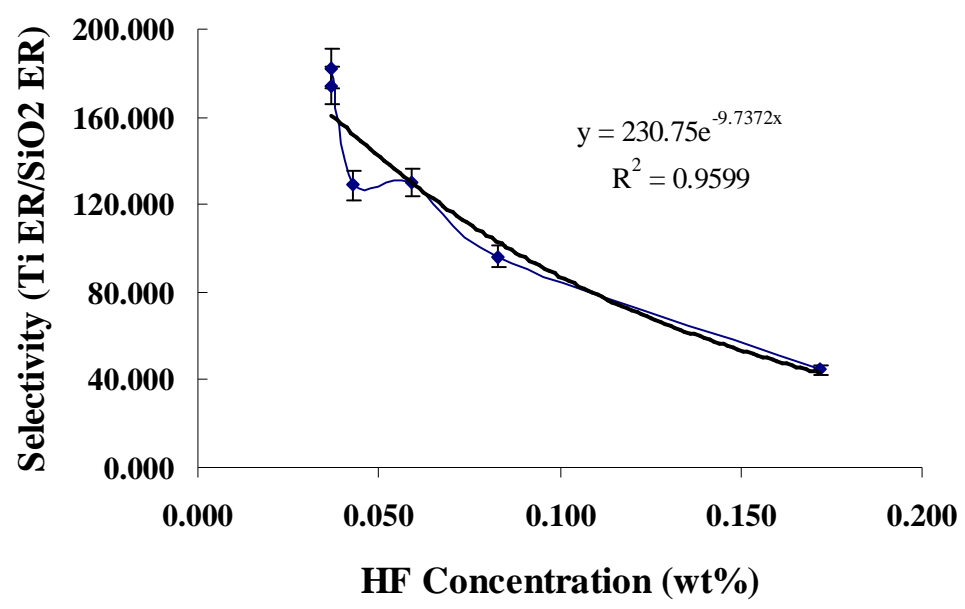

Figure 6.16 $\mathrm{Ti} / \mathrm{SiO}_{2}$ selectivity with Respect to $\mathrm{HF}$ Concentration.

\subsection{Model for Ti etch Selectivity}

Through careful interpretation of the analytical data, a plausible mechanism pertaining to the selectivity of Ti etching process may be rationalized. The NMR and Mass spectroscopic results clearly indicate that $\mathrm{F}$ is attached to $\mathrm{Si}$ monomers, $\mathrm{Si}-\mathrm{O}-\mathrm{Si}$ oligomers (dimers, trimers, etc...), and (-Si-O-)n polymers backbone. What ever remaining small concentration of $\mathrm{HF}$ in equilibrium with $\mathrm{Si}$ species is insufficient to cause $\mathrm{SiO}_{2}$ etching. One model to rationalize the Ti selectivity of the formulation is in terms of a catalytic role provided by titanium surface for the release of $\mathrm{F}$ into the solution and/or the Ti surface in particular. This desirability in releasing the fluorine onto the Ti surface can be attributed to two likely motives. The first is due to the greater difference in electronegativity between F-Ti vs. $\mathrm{Si}$ or $\mathrm{SiO}_{2}(\mathrm{~F}-\mathrm{Si}$ and $\mathrm{F}-\mathrm{O})$, the 
second is the abundance of $\mathrm{H}^{+}$on the surface (note, the $\mathrm{pH}$ of the formulation is $~ 2$ ) which promotes the formation of $\mathrm{HF}$ and/or $\mathrm{HF}_{2}^{-}$responsible for etching the Ti. To further understand the selective etching model, one must revisit the basic concepts of corrosion since Ti etching is a corrosion process. Metal dissolves by liberating electrons into the bulk of the metal which migrate to the adjoining surface, where they react with $\mathrm{H}^{+}$in the solution to form $\mathrm{H}_{2}$. The dissolution of Ti in the wet etch/clean formulation is shown schematically in figure 6.17. The formation of HF mechanism which rapidly reacts with $\mathrm{Ti}$ is illustrated. This release of $\mathrm{F}$ onto the Ti surface is much more favorable than on $\mathrm{SiO}_{2}$ due to electronegativity differences and the presence of $\mathrm{H}^{+}$on the Ti surface. These conditions provide the necessary requirements for the release of fluorine from the organosilicon monomers and oligomers and the formation of HF on the Ti surface. A similar model based on dissolution of $\mathrm{TiO}_{2}$ can be advanced. However, to discriminate between these two models would require further electrochemical corrosion studies. 


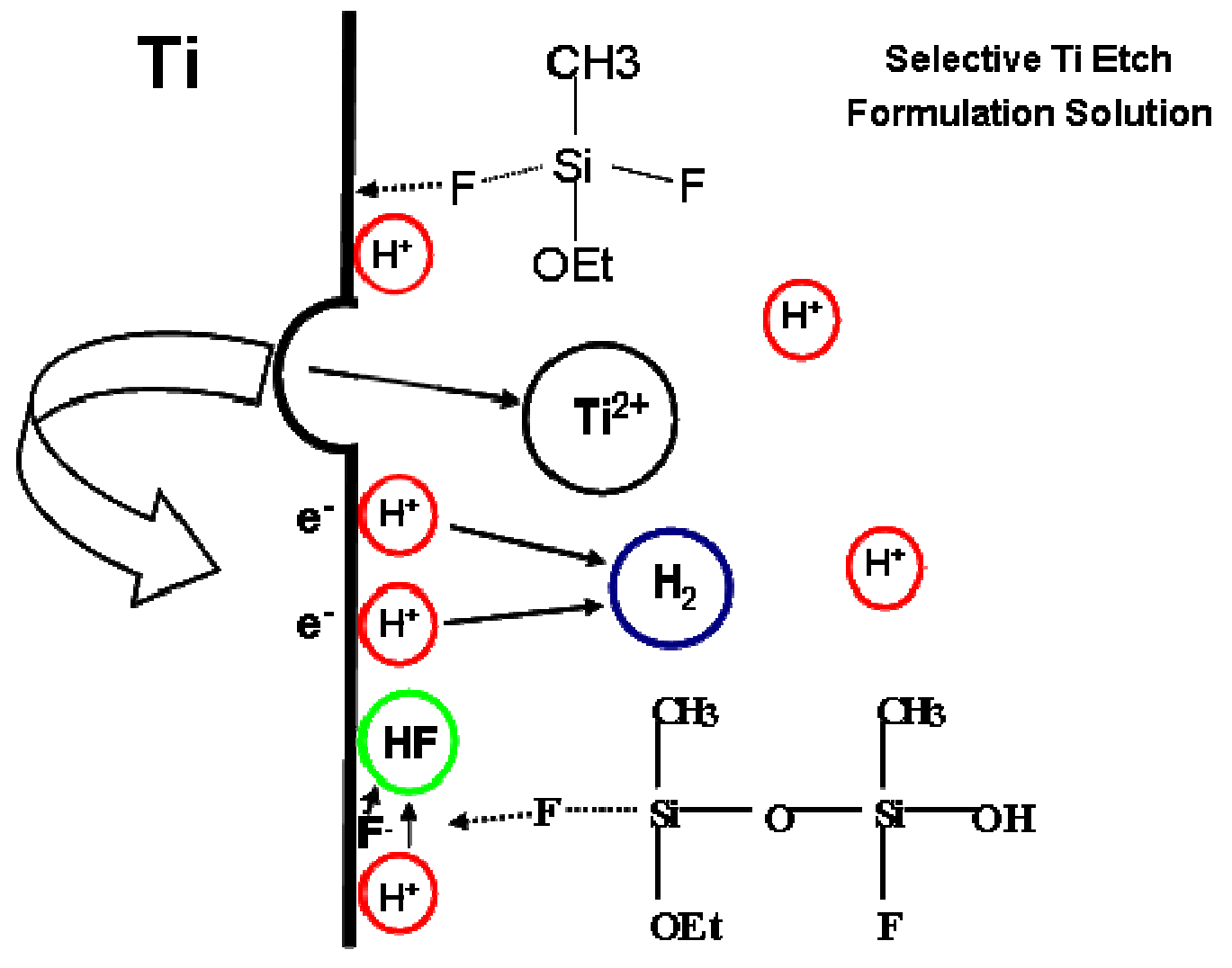

Figure 6.17 Schematic diagram of Ti dissolution in the wet etch/clean formulation.

\subsection{Summary}

NMR showed multiple peaks by ${ }^{1} \mathrm{H}$ NMR that corresponded to different methyl groups, while ${ }^{19} \mathrm{~F}$ NMR also showed multiple broad peaks. GC-MS work indicated that there were discrete correlations of peaks in the GC to fragmentation patterns in the MS which further showed fragmentation patterns that were common to different molecules. The MALDI work supports GC-MS findings. IR studies confirmed Si-F covalent bonding. BTA concentration was determined quantitatively and its behaviors were characterized using HPLC-UV/VIS-MS. HRICP-MS method was successfully employed to determine $\mathrm{Si}$ concentration in the formulation for characterization purposes. ELSD-MS technique was used to complement other 
analytical methods in identifying the various oligomer/polymer species. Interpretation of the data suggests that the formulation is composed of a mixture of monomers, dimers, trimers, to hexamers siloxane compounds containing methyl, fluoro, hydroxyl, and ethoxy groups. Discrete monomers of similar composition are also likely. The following is a representation of these compounds.<smiles>CCO[Si](C)(F)P</smiles><smiles>C[Si](F)(F)(F)F</smiles><smiles>CCO[Si](C)(F)OCC</smiles><smiles>CC[Si](C)(F)F</smiles><smiles>CCO[Si](C)(O)(O)(O)c1ccccc1</smiles><smiles>C[Si](O)(O)F</smiles><smiles>CCO[Si](C)(F)O[Si](C)(O)F</smiles><smiles>CC(C)(C)[Si](C)(F)O[Si](C)([O-])O</smiles><smiles>CC(C)(O)[Si](C)(O)O</smiles><smiles>[3H][Si](C)(O)O[Si](C)(O)F</smiles><smiles>[3H][Si](C)(O)O[Si]([3H])(C)O[Si](C)(O)O[Si]([3H])(C)O[Si]([3H])(C)O[Si](C)(C)OCC</smiles> 
The reaction products, in conjunction with the residual HF, provide a selective Ti etching capability. The reaction orders with respect to Ti etching and $\mathrm{SiO}_{2}$ etching rates of HF were determined and were found to be 1 and 1.8 respectively. The $\mathrm{pH}$ of the formulation and the aerobic conditions in which the Ti etching is carried out offers protection with respect to tungsten etching. This is a distinct advantage as $\mathrm{W}$ is a common element present at first layer of interconnects. The Pourbaix diagram for tungsten (Figure 4.5) shows the formation of passivating $\mathrm{WO}_{3}$ film at low OCP and $\mathrm{pH}$ of $\sim 2$. Thus the overall formulation achieves selective etching with respect to $\mathrm{Ti}$ while maintaining negligible etch rates with respect to $\mathrm{SiO}_{2}$ and $\mathrm{W}$. 


\section{CHAPTER 7}

\section{Scalability and Process Control}

\subsection{Introduction}

The objective of this chapter is to discuss the strategy executed at the various stages of scalability development and optimizations of the wet etch/clean formulation synthesis for high volume manufacturing. Two key strategies were exploited in establishing an optimal apparatus design to carry out the synthesis that accommodates the needs of manufacturing Fabs: 1) Employing the 60 letter bath in the automated wet station where wafers are processed as a reaction vessel; 2) Designing a reactor in which the synthesis can be carried out externally and on a much larger scale than the first approach.

The scalability of the formulation synthesis from a laboratory-scale $(200 \mathrm{ml}$ in beaker) to high volume manufacturing batch reactor (3000 gallons) went through a steep learning curve due to difficulties related to the synthesis environment. Besides the factors previously discussed (temperature, time, MTES addition rate, HF/MTES ratio, and stirring rate), additional variables related to the large volume and the enclosed reactor system had to also be addressed and overcome. The most notable challenge was related to the fact that the synthesis in a beaker is an open system, while all other stages of volume scaling dealt with an enclosed system. Some of the key issues that were readily observed as a result of employing an enclosed system were a significant shift in $\mathrm{pH}$ and an increased susceptibility to forming polymer, particles, 
and oily residue (i.e. gel). These shortcomings have considerably impacted the formulation from a reliability and functionality stand point. In this scalability development work at various stages, extensive experimental design was carried out in response to the increased number of factors resulting from the enclosed system. These additional factors included nitrogen sweep rate, pump flow rate, and the longer time required for the synthesis. An optimal nitrogen sweep is required at the head space of the reactor to assist in the escape of volatile species and prevent their condensation. The system differences open (beaker) vs. enclosed (reactor) lead to adopting significant changes with respect to previously optimized reaction conditions in labscale synthesis. Previous and new factors were thoroughly investigated throughout the scalability development in which a successful formulation was demonstrated at each stage of development.

Here, some reaction products were carefully characterized in an attempt to establish a capability that provides real time information with respect to synthesis progress. This ability ensures that proper synthesis conditions are met (i.e. real-time process evaluation and quality control) and necessary adjustments to reaction conditions can be made to ensure that synthesis is following a proper path. Typically, analytical techniques are needed to serve as a predictive indicator of product worthiness which are required for the certification of chemical formulations, particularly, if they are to be implemented for high volume manufacturing. 
Typically, analytical techniques are needed to serve as a predictive indicator of product worthiness which are required for the certification of chemical formulations, particularly, if they are to be implemented for high volume manufacturing.

\subsection{Systems Designs and Assembly}

\subsubsection{Synthesis in the Bath within the Automated Wet Station}

Figure 7.1 illustrates the components of the system that was utilized to synthesize the formulation.

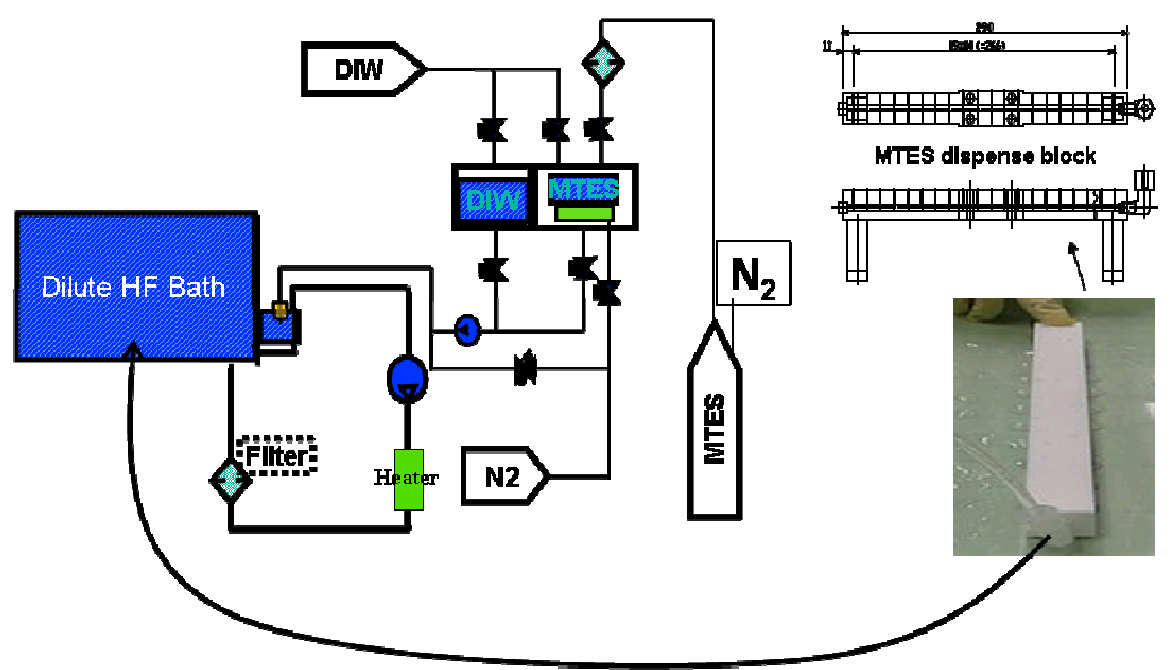

Figure 7.1 Schematic of the formulation synthesis system within the automated wet station. Dilute HF bath and the MTES delivery components are shown.

The 60 liter bath which is used to process wafers on an automated wet station and the various components to deliver the MTES to the bath are shown in Figure 7.1. It shows the dispense block used to inject the MTES into the bottom of the dilute HF bath. This system was used as a temporary solution until the high volume manufacturing (HVM) reactor was constructed.

A Schematic diagram of the $3000 \mathrm{HVM}$ reactor and its components is shown in Figure 7.2. The system features the reactor, MTES container and delivery system, 
recirculation pump, and heat exchanger. Furthermore, a complete on-line monitoring system was put in place to monitor temperature, pump pressure, solution flow rate, and nitrogen mass flow rate.

A number of milestones were achieved throughout the design and scalability development. In the first stage, a 20 gallon reactor referred to as sample system was assembled. In the second stage of development a 120 gallon pilot system was designed and constructed. In the third stage of scalability a 1000 gallon reactor was erected, and finally in the last phase a 3000 gallon reactor was built.

The reaction synthesis in the reactor was carried out in a manner similar to that in the beaker. The dilute HF solution was prepared using a concentrated $49 \% \mathrm{HF}$. This solution is then heated to $80^{\circ} \mathrm{C}$. Pure MTES is added at a slow rate to the dilute HF solution at $80{ }^{\circ} \mathrm{C}$. Throughout the synthesis, the temperature is maintained at 80 ${ }^{\circ} \mathrm{C}$ and vigorous mixing is applied by employing a pump. For the reaction to reach completion (equilibrium) a period much longer than the 2 hours in beaker was required. In addition to altering reaction parameters and conditions, a new $\mathrm{SiO}_{2}$ etch rate target of $0.3 \mathrm{~A}^{\circ} / \mathrm{min}$ was established to alleviate the limitations given by the enclosed reactor system. 


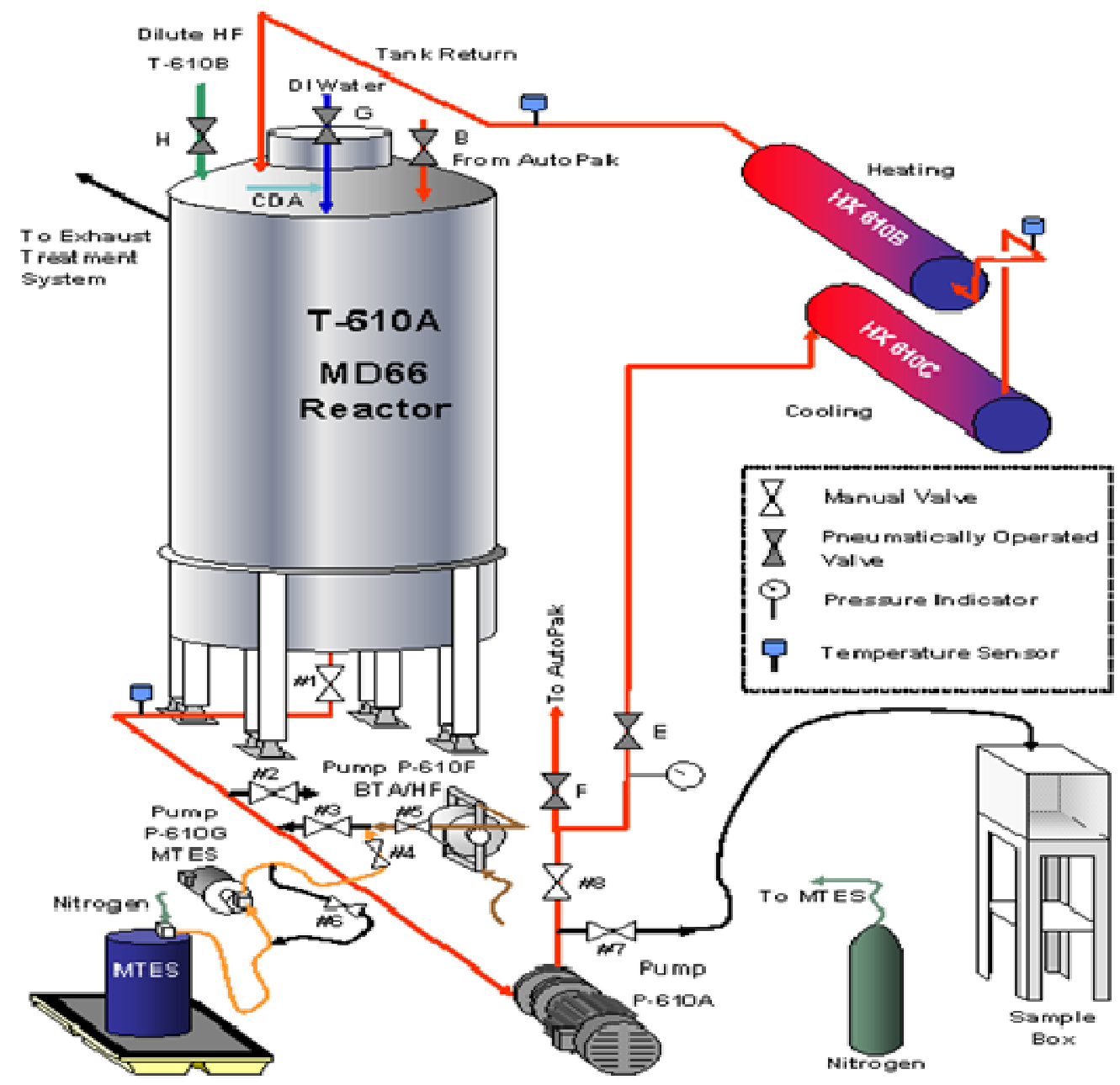

Figure 7.2 Schematic diagram of 3000 gallon reactor and its components.

Functional testing of the final reaction product was done on blanket test wafers. The etch rate experiments were conducted in the same manner as previously described in chapter 5. Additional key parameters of the reaction products were analyzed and their concentrations were determined. $\mathrm{HF}, \mathrm{F}^{-}$, and BTA concentrations were determined at the end of each synthesis as an indication of a successful synthesis and to establish a base line. Note that manipulating any of these process parameters enough to drift $\mathrm{F}^{-}$concentration would push the CVD etch rate out of control limits. 


\subsection{Process Control and Stability Results and Discussion}

The syntheses model with respect to HF concentration and silicon oxide etch rate as a function of time is shown in Figure 7.3. Note the longer time required to reach equilibrium conditions and stabilize the $\mathrm{SiO}_{2}$ etch rate to achieve the required target.

\section{Synthesis Model}

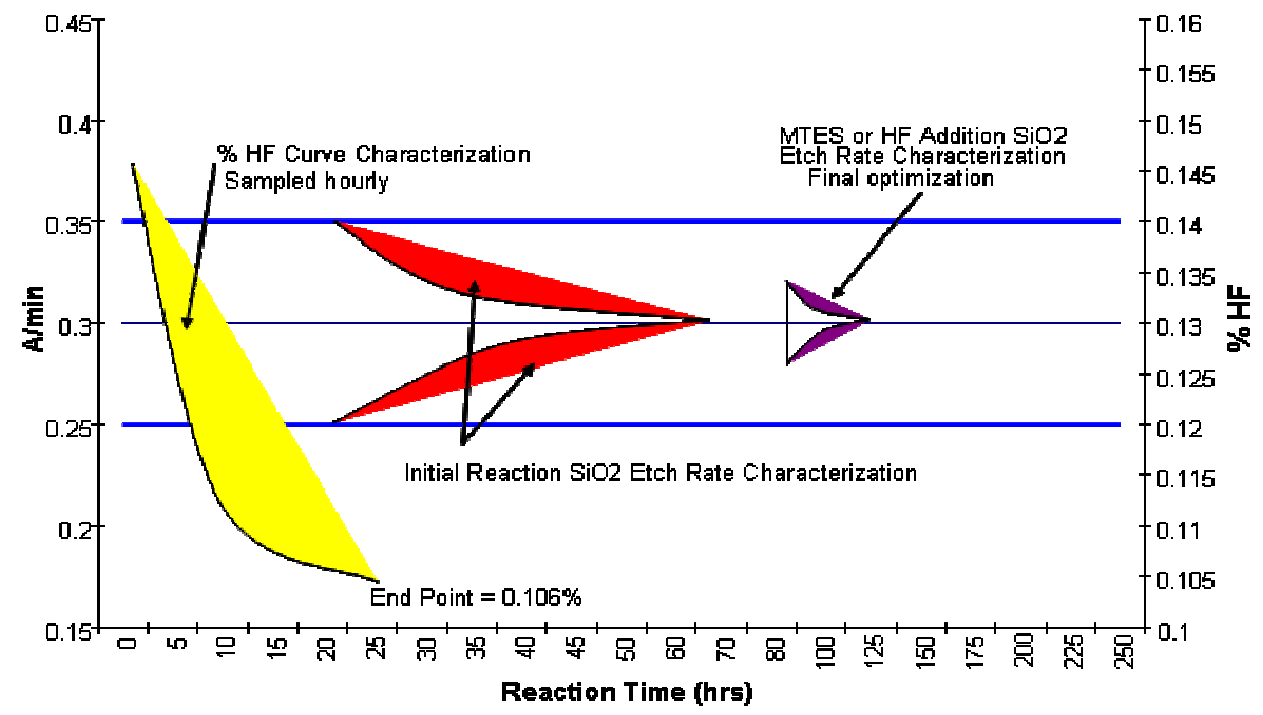

Figure 7.3 The syntheses model with respect to HF concentration and silicon oxide etch rate.

The reaction profile in the reactor at the various phases of scalability is shown in Figure 7.4. The plot clearly shows the ability to match the synthesis with respect to HF concentration trend at each phase of scalability. This is a good indication of progressing in an acceptable path at each stage of scaling and reactor assembly development. For the 1000 and 3000 gallon reactors, synthesis control and batch to batch uniformity is demonstrated as shown in the process control charts illustrated in Figures 7.5, 7.6, and 7.7 for $\mathrm{SiO}_{2}, \mathrm{HF}$ concentration, and $\mathrm{F}^{-}$concentration respectively. 
Additional indicators were investigated as well, these included BTA concentration, and Ti ER in which process control was also demonstrated. Ti stripping was shown to be robust and consistent from batch to batch with etch rate of $\sim 120 \mathrm{~A} / \mathrm{min}$.

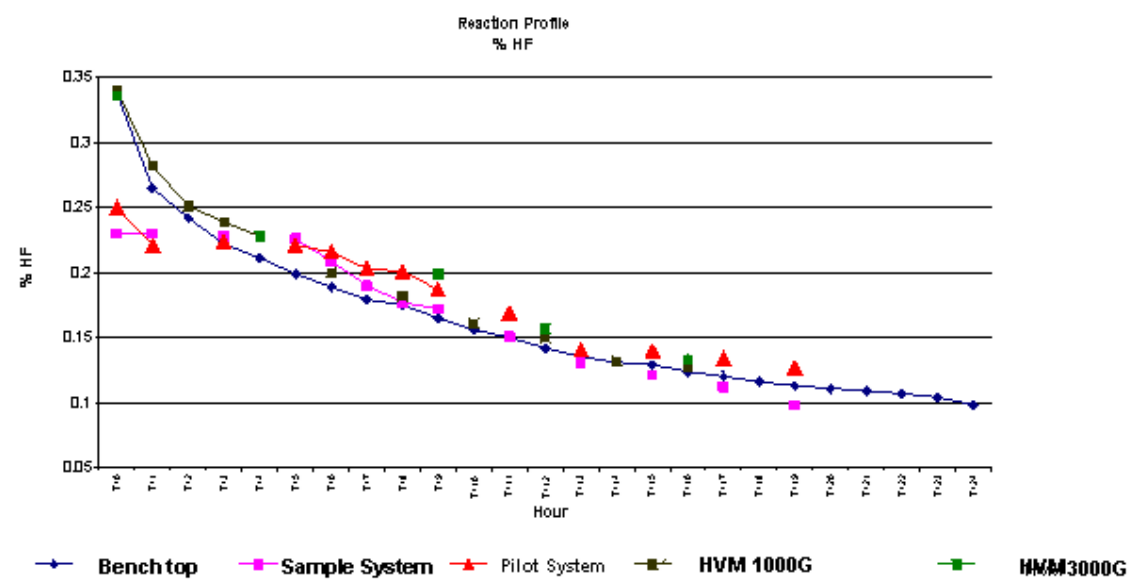

Figure 7.4 Reaction profile with respect to $\mathrm{HF}$ concentration and reaction time at various stages of scalability.

In comparison with the data for the open beaker synthesis, as shown in table 6.1, the $\mathrm{SiO}_{2}$ etch rates are higher at the equivalent concentration of $\mathrm{HF}$ in the closed reactor system this could arise from the evaporative loss of reactive fluorine containing components in the open system. Note significantly different time dependences of HF concentration in open (Figure 6.14) and close system (Figure 7.4). 


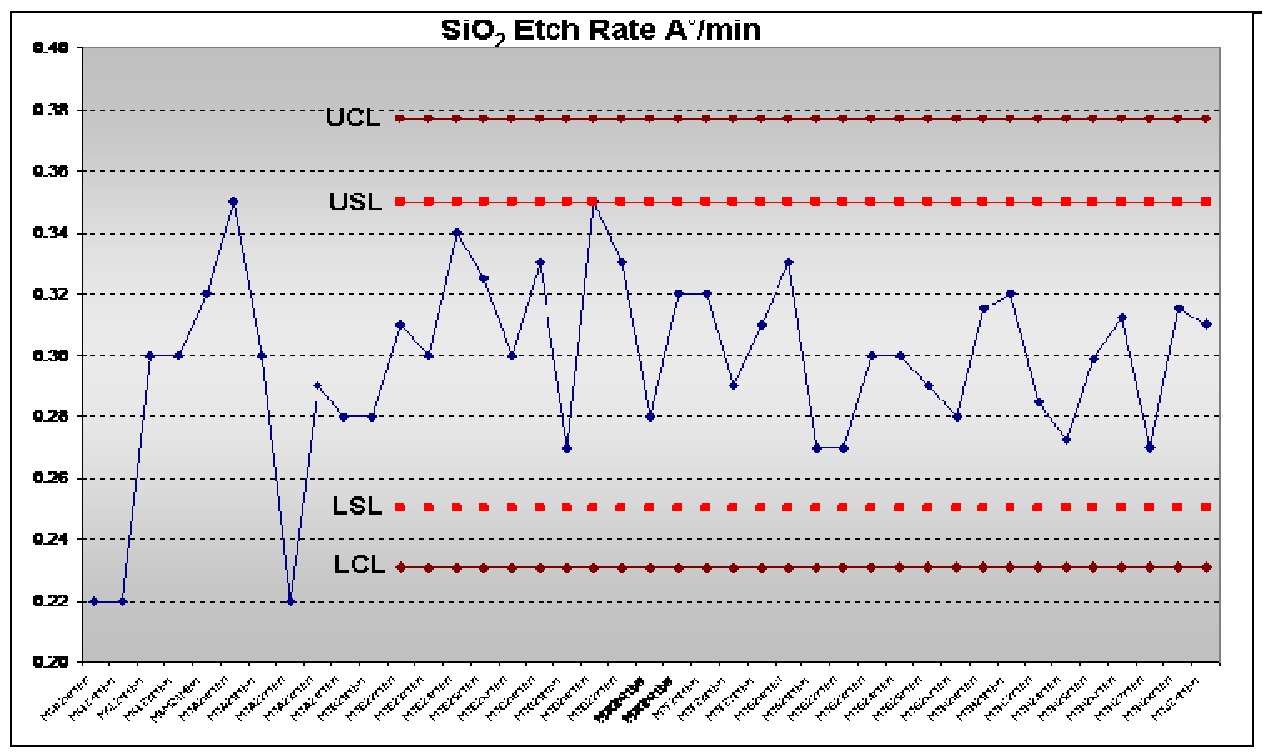

Figure 7.5 $\mathrm{SiO}_{2}$ etch rate process control charts for various batches from the reactor.

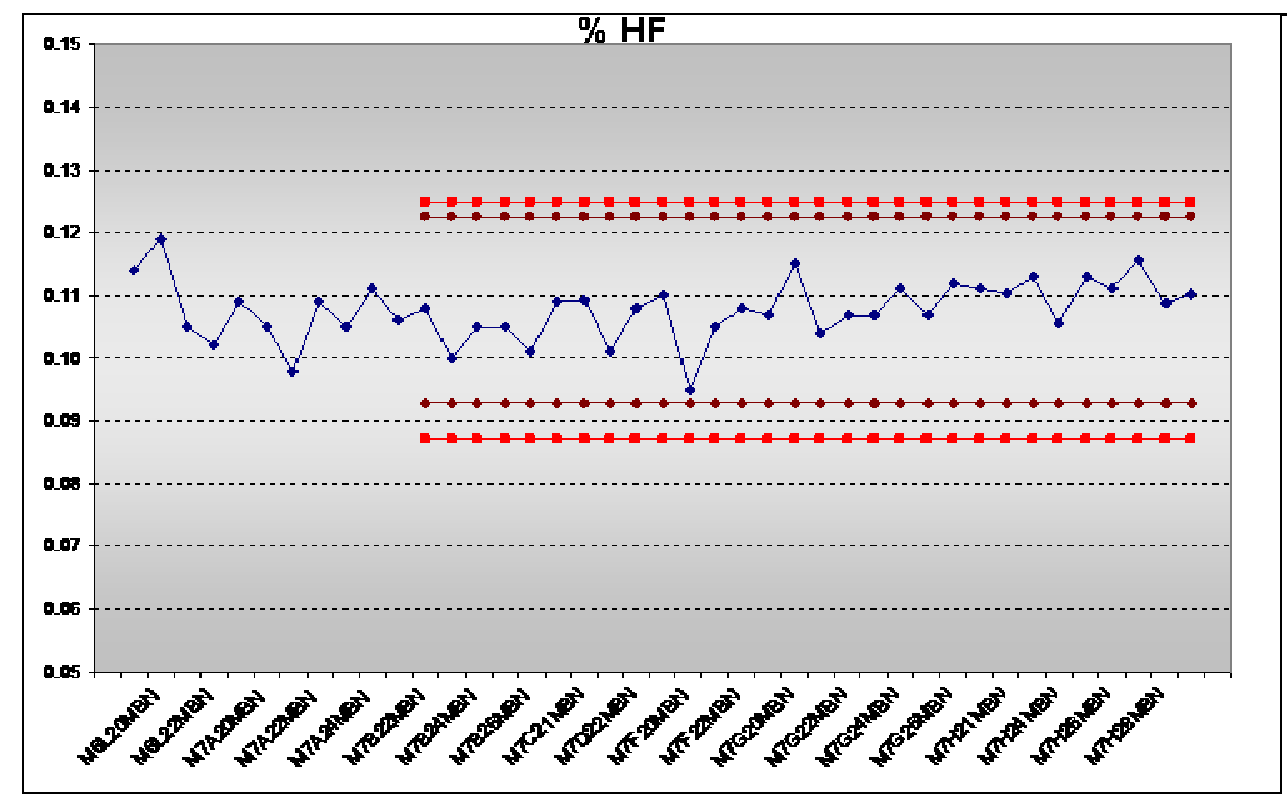

Figure 7.6 HF concentration process control charts for various batches from the reactor. 


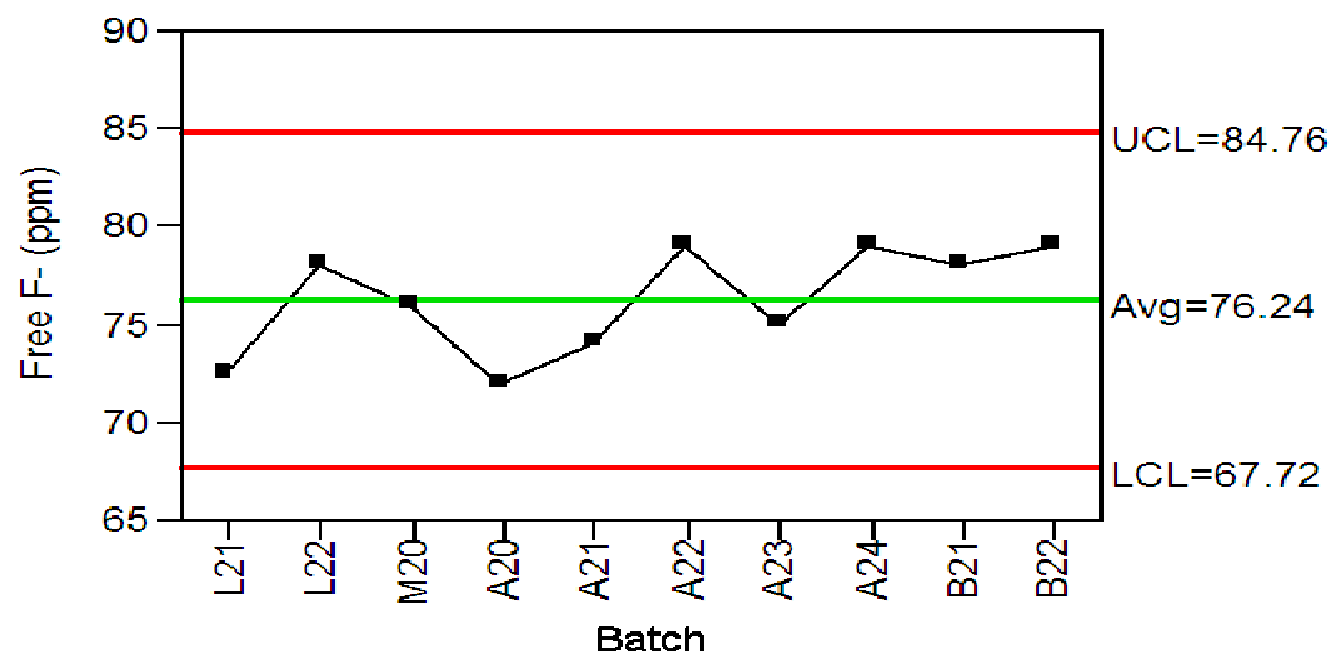

Figure 7.7 $\mathrm{F}^{-}$Concentration process control chart for a number of batches.

\subsection{Summary}

The synthesis of wet etch/clean formulation on a large scale in the reactor was demonstrated as suggested by the summarized results. The formulation developed met necessary success criterion described previously in which a homogenous solution mixture free of particles, polymer, or oily residue is created. Furthermore, etch rate targets of the various substrates were achieved and was confirmed on patterned wafers.

The kinetic work discussed in chapter 6 was also important in developing a successful synthesis. The concentrations of $\mathrm{H}_{2} \mathrm{SiO}_{3}$ and $\mathrm{H}_{2} \mathrm{SiF}_{6}$ were investigated as a function of synthesis time, which assisted in providing additional gauge to characterize and achieve a successful synthesis.

For the scalable reactor design, potential scale-up obstacles were identified and addressed throughout the various phases of development. The scalability development and investigation work revealed that the removal of the by-product volatile species using $\mathrm{N}_{2}$ sweep and the modification of a number of factors from the beaker scale reaction was a key for an optimal and successful synthesis. A shelf life stability study 
was undertaken to determine the extent of the formulation stability. This investigation indicated that the compositional stability of the formulation exceeds a period longer than one year. This stability could be attributed to maintaining low $\mathrm{pH}$ where $\mathrm{Si}$ monomeric species dominate and polymerization is suppressed under these conditions $^{226}$. 


\section{CHAPTER 8}

\section{Conclusions and Future Work}

\subsection{Conclusions}

As multistep, multilayer processing in semiconductor industry becomes more complex, the role of cleaning solutions and etching chemistries are becoming important in enhancing yield and in reducing defects. This thesis demonstrates successful formulations that exhibit copper and tungsten compatibility, and capable of ILD cleaning and selective Ti etching.

The synthesis of wet etch/clean formulation on a large scale in a reactor (3000 gallons) was demonstrated as suggested by the summarized results. The formulation developed met necessary success criterion described previously in which a homogenous solution mixture free of particles, polymer, or oily residue is created. Furthermore, etch rate targets of the various substrates were achieved and was confirmed on patterned wafers.

In the copper corrosion work, Potentiodynamic polarization experiments carried out in the organic, HF containing cleaning solution, show active, activepassive, passive, and transpassive regions. Use of organic corrosion inhibitors, deareated solutions and possible use of $\mathrm{H}_{2} \mathrm{O}_{2}$ provides an effective method to reduce corrosion rates and copper concentration in cleaning solution. $\mathrm{H}_{2} \mathrm{O}_{2}$ addition to the cleaning solution appears to be very beneficial by creating a $\mathrm{CuO}$ film that is resistant to attack by $\mathrm{HF}$. The incorporation of $\mathrm{H}_{2} \mathrm{O}_{2}$ provides an additional advantage by 
enhancing the cleaning solution's ability in removing particles and residue. Furthermore, a simple kinetic model is proposed for copper dissolution during wet chemical etch process. It predicts a first order kinetics with respect to dissolved oxygen and HF, the two key ingredients involved in corrosion of copper. If dissolved oxygen is eliminated or if protective $\mathrm{CuO}$ is formed the rate of copper corrosion can be suppressed.

The developed $\mathrm{Ti}$ selective wet etch/clean formulation exhibits the ability to remove the plasma etch polymer residue, and strip patterned $\mathrm{Ti}$ thin film layer while suppressing the etch rates of $\mathrm{SiO}_{x}, \mathrm{CDO}, \mathrm{Si}_{3} \mathrm{~N}_{4}, \mathrm{SiC}, \mathrm{Cu}, \mathrm{W}$. This capability was demonstrated on blanket and DD patterned wafers with no evidence of any adverse impact to interconnect and transistor structures or underlying materials. The results for patterned wafers are in agreement with the blanket wafers etch rates findings for the various substrates.

The concept of incorporating a silane cross-linking compound into a dilute HF solution is novel. It was hypothesized that silicon precursors may hinder the HF ability to etch silicon oxide and other silicon oxides dielectric materials. This phenomenon can be ascribed to the formation of products such as salicic acid or possibly other species that if present at sufficient amounts in the solution, would establish an equilibrium with $\mathrm{SiO}_{\mathrm{x}}$. Once an equilibrium is reached, silicon oxide and similar materials are no longer etched by HF. Another plausible mechanism is the formation of species that may have passivated the silicon oxide surface. 
The novel feature of this formulation is that although it contains HF, a common agent for etching $\mathrm{SiO}_{\mathrm{x}}$, it does not attack ILD or $\mathrm{SiO}_{2}$ materials. The use of HF was considered high risk. This formulation represents a first of its kind in meeting demanding requirements in selective metal HM etching in the semiconductor industry and enables the sub $50 \mathrm{~nm}$ and beyond process technologies for advanced ULSI. Interpretation of the data suggests that the formulation is composed of a mixture of monomers, dimers, trimers, to hexamers siloxane compounds containing methyl, fluoro, hydroxyl, and ethoxy groups. Discrete monomers of similar composition are also likely.

The reaction products, in conjunction with the residual HF, provide a selective Ti etching capability. The reaction orders with respect to $\mathrm{Ti}$ etching and $\mathrm{SiO}_{2}$ etching rates of $\mathrm{HF}$ were determined and were found to be 1 and 1.8 respectively. The $\mathrm{pH}$ of the formulation and the aerobic conditions in which the Ti etching is carried out offers protection with respect to tungsten etching. This is a distinct advantage as $\mathrm{W}$ is a common element present at first layer of interconnects. The Pourbaix diagram for tungsten (Figure 4.5) shows the formation of passivating $\mathrm{WO}_{3}$ film at low $\mathrm{OCP}$ and $\mathrm{pH}$ of $\sim 2$. Thus the overall formulation achieves selective etching with respect to $\mathrm{Ti}$ while maintaining negligible etch rates with respect to $\mathrm{SiO}_{2}$ and $\mathrm{W}$.

The kinetic work discussed in chapter 6 was important in developing a successful synthesis. The concentrations of $\mathrm{H}_{2} \mathrm{SiO}_{3}$ and $\mathrm{H}_{2} \mathrm{SiF}_{6}$ were investigated as a function of synthesis time, which assisted in providing additional gauge to characterize and achieve a successful synthesis. 
The excellent electrical performance of the circuit further demonstrated the Ti etch/clean formulation's capability. A tight distribution obtained in via resistance chain was demonstrated, suggesting that the formulation is suitable for this application.

For the large volume reactor design, potential scale-up obstacles were identified and addressed throughout the various phases of development. The scalability development and investigation work revealed that the removal of the byproduct volatile species using $\mathrm{N}_{2}$ sweep and the modification of a number of factors from the beaker scale reaction was a key for an optimal and successful synthesis. A shelf life stability study was undertaken to determine the extent of the formulation stability. This investigation indicated that the compositional stability of the formulation exceeds a period longer than one year.

\subsection{Future Work}

\subsubsection{Cleanability Improvements}

Even though he developed formulation has been successful for this application, further improvements with respect to cleanability can be pursued. Studies geared towards improvements in wetting the patterned ILD surface should be conducted. This may involve the use of surface tension reducing components.

\subsubsection{Further Compositional and Kinetic Characterization}

Further characterization to determine the exact composition of the formulation needs to be performed. Effect of dissolved oxygen and $\mathrm{pH}$ needs to be explored systematically. 


\section{References}

${ }_{1}^{1}$ J. Li, Y. Shacham-Diamand, J. W. Mayer, Materials Science Reports 9, 1 (1992).

${ }^{2}$ S. P. Murarka, S. W. Hymes, Critical Reviews in Solid State and Materials Sciences 20, 87 (1995).

${ }^{3}$ D. Edelstein, J. Heidenreich, R. Goldblatt, W. Cote, C. Uzoh, N. Lustig, P. Roper, T. McDevitt, W. Motsiff, A. Simon, J. Dukovic, R. Wachnik, H. Rathore, R. Schulz, L. $\mathrm{Su}, \mathrm{S}$. Luce, J. Slattery, International Electron Device Meeting Technical Digest 773, Dec. (1997).

${ }^{4}$ S. Venketesan, A. V. Gelatos, V. Misra, B. Smith, R. Islam, J. Cope, B. Wilson, D. Tuttle, R. Cardwell, S. Anderson, M. Angyal, R. Bajaj, C. Capasso, P. Crabtree, S. Das, J. Farkas, S. Filipiak, B. Fiordalice, M. Freeman, P. V. Gilbert, M. Herrick, A. Jain, H. Kawasaki, C. King, J. Klein, T. Lii, K. Reid, T. Saaranen, C. Simpson, T. Sparks, P. Tsui, R. Venkatraman, D. Watts, E. J. Weitzman, R. Woodruff, I. Yang, N. Bhat, G. Hamilton, Y. Yu, International Electron Device Meeting Technical Digest 769, Dec. (1997).

${ }^{5}$ N. Rohrer, C. Akrout, M. Canada, D. Cawthron, B. Davari, R. Floyd, S. Geissler, R. Goldblatt, R. Houle, P. Kartschoke, D. Kramer, P. McCormick, G. Salem, R. Schulz, L. Su, L. Whitney, International Solid-State Circuits Conference Technical Digest 240, Feb. (1998).

${ }^{6}$ D. Edelstein, J. Heidenreich, R. Goldblatt, W. Cote, C. Uzoh, N. Lustig, P. Roper, T. McDevitt, W. Motsiff, A. Simon, J. Dukovic, R. Wachnik, H. Rathore, R. Schulz, L. Su, S. Luce, J. Slattery, International Electron Device Meeting Technical Digest 773, Dec. (1997).

${ }^{7}$ T. Licata, E.G. Colgan, J. M. Harper, S. E. Luce, IBM J. Res. Develop. 39, 419 (1995).

${ }^{8}$ G. S. Oehrlein, G. J. Scilla, S. Jeng, Appl. Phys, Lett. 52, 907 (1988).

${ }^{9}$ G. S. Oehrlein, Y.H. Lee, J. Vac. Sci. Technol. A4, 1585 (1987).

${ }^{10}$ D. K. Schroder, Semiconductor Materials and Device Characterization, John Wiley \& Sons, Inc., New York, (1990).

11 A. Somashekhar, H. Ying, P. B. Smith, D. B. Aldrich, R. J. Nemanich, J. Electrochemical Society 146, 2318 (1999).

${ }^{12}$ Q. Han, B. White, I. L. Berry, C. Waldfried, O. Escorcia, Solid State Phenomena. 103, 341 (2005).

${ }^{13}$ U. Kazuyoshi, M. D. Vincent, K. Takamaro, J. Electrochem. Soc. 144, 2565 (1997).

${ }^{14}$ M. Hussein, R. Brain, R. Turkot, J. Leu, V. Singh, S. Sivakumar, “ Dual damascene patterning of polymer interlayer dielectrics", in Proc. of the International Interconnect Technology Conf. (San Francisco, CA.), IEEE Press, Piscataway, N.J., pp. 33 June 2-4, (2003).

${ }^{15}$ T. Furusawa, S. Machida, D. Ryuzki, K. Sameshima, T. Ishida, K. Ishikawa, N. Miura, N. Konishi, T. Saito, H. Yamaguchi; "Novel dissoluble hardmask for damageless Cu/Low-k interconnect fabrication", in Proc. of the International Interconnect 
Technology Conf. (San Francisco, CA), IEEE Press, Piscataway, N. J., pp. 195 June 24, (2003).

${ }^{16}$ P. A. Kohl, D. M. Bhusari, M.Wedlake, C. Case, F. P. Klemens, J. Miner, L. Byung-Chan, R. J. Gutmann, R. Shick, Electron Device Lett. 21, 557 (2000).

${ }^{17}$ J. J. Waeterloos, E. O. Shaffer II, T. Stokich Jr., J. Hetzner, D. Price, L. Booms, R. A. Donaton, G. Beyer, B. Coenegrachts, R. Caluwaerts, H. Struyf, Z. S. Tokei, I. Vervoort, B. Sijmus, I. Vos, K. Maex, T. Komiya, M. Iwashita, "Integration of a low permittivity spin-on embedded hardmask for Cu/SiLK resin dual damascene," in Proc. IEEE Int. Interconnect Technol. Conf., pp. 60-62 Jun. 4-6, (2001).

${ }^{18}$ M. Hussein, R. Brain, R. Turkot, J. Leu, V. Singh, S. Sivakumar, "Dual damascene patterning of polymer interlayer dielectrics," in Proc. Int. Interconnect Technology Conf. Piscataway, NJ, Jun. 2-4, pp. 33-35 (2003).

${ }^{19}$ R. Kammura, VLSI Technology Symposium, 108 (2003).

${ }^{20}$ H. Miyajima, proceedings Of IEDM. 329 (2004).

${ }^{21}$ N. Nakamura, proceedings of IITC. 228 (2004).

${ }^{22}$ E. Braun, Semicond. Int. (2004).

${ }^{23}$ E. Braun, Semiconductor International (2004).

${ }^{24}$ Mei Qi Weng, Miao Chun Lin, Joe Lai, Ren Huang, J.H. Liao, "Metal hard mask employed Cu/low $k$ post etch resist ash/wet clean process optimization and integration into 65nm manufacturing flow" Ultra Clean Process OF Semiconductor Surfaces, UCPSS, pp.75-76, (2006).

${ }^{25}$ Semiconductor Industry Association (SIA), The International Technology Roadmap for

Semiconductors, Interconnect (2001).

${ }^{26}$ W. Kern, D.A. Puotinen, RCA review, 187, (1970).

${ }^{27}$ W. Kern, ed. Handbook of Semiconductor Cleaning Technology. Park Ridge, NJ: Noyes Publications (1993).

${ }^{28}$ Intel website, http://www.intel/research/silicon/mooreslaw.htm, Sept. (2002).

${ }^{29}$ M. Bohr, Proceedings of International Electron Devices Meeting, p. 241 (1995).

${ }^{30}$ IBM Journal of Research and Development, 39(4), 189 (1995).

31 International Technology Roadmap for Semiconductors, 2001 Edition. Semiconductor Industry Association, Interconnects section, p. 4 (2001).

32 J. G. Ryan, J. E. Heidenreich, W. J. Cote, R. M. Geffken, T. N. Theis, Advanced Metallization and Interconnect Systems for ULSI Applications in 1997 Conference Proceedings, pp. 138, Oct. (1997).

${ }^{33}$ T. J. Licata, E. G. Colgan, J. M. E. Harper, S. E. Luce, IBM Journal of Research and Development 39(4), 419 (1995).

${ }^{34}$ C. Ryu, K. W. Kwon, A. L. S. Loke, V. M. Dubin, R. A. Kavari, G. W. Ray, S. S. Wong, Symposium on VLSI Technology Technical Digest, pp. 156, June (1988).

${ }^{35}$ R. W. Mann, L. A. Clavenger, P. D. Agnello, F. R. White, IBM Journal of Research and Development 39(4), 403 (1995).

${ }^{36}$ T. J. Licata, E. G. Colgan, J. M. E. Harper, S. E. Luce, IBM Journal of Research and Development 39(4), 419 (1995). 
37 I. Ames, F. M. d'Heurle, R. E. Horstmann, IBM Journal of Research and Development, 14, 461 (1970).

${ }^{38}$ M. Bohr, S. S. Ahmed, S. U. Ahmed, M. Bost, T. Ghani, J. Greason, R. Hainsey, C. Jan, P. Packan, S. Sivakumar, S. Thompson, J. Tsai, S. Yang, International Electron Device Meeting Technical Digest 847 (1996).

${ }^{39}$ M. J. Hampden-Smith, T. T. Kodas, MRS Bulletin 18(6), 39 (1993).

${ }^{40}$ J. S. H. Cho, "Process integration issues of copper interconnections for ULSI," Ph.D. Thesis, Stanford University, Mar. (1994).

${ }^{41}$ R. S. Muller, T. I. Kamins, Device Electronics for Integrated Circuits, 2nd edition. New York, NY: John Wiley \& Sons, pp. 156, (1986).

42 J. D. McBrayer, R. M. Swanson, T. W. Sigmon, Journal of the Electrochemical Society 133(6), 1242 (1986).

${ }^{43}$ D. Edelstein, J. Heidenreich, R. Goldblatt, W. Cote, C. Uzoh, N. Lustig, P. Roper, T. McDevitt, W. Motsiff, A. Simon, J. Dukovic, R. Wachnik, H. Rathore, R. Schulz, L. Su, S. Luce, J. Slattery, International Electron Device Meeting Technical Digest 773 (1997).

${ }^{44}$ J. G. Ryan, R. M. Geffken, N. R. Poulin, J. R. Paraszczak, IBM Journal of Research and Development 39(4), 371 (1995).

${ }^{45}$ J. G. Ryan, J. E. Heidenreich, W. J. Cote, R. M. Geffken, T. N. Theis, Advanced Metallization and Interconnect Systems for ULSI Applications in 1997 Conference Proceedings, pp. 399, Oct. (1997).

${ }^{46}$ M. M. Chow, J. E. Cronin, W. L. Guthrie, C. W. Kaanta, B. Luther, W. J. Patrick, K. A. Perry, C. L. Standley, U.S. Patent 4,789,648, issued 6 Dec. (1988).

${ }^{47}$ C. W. Kaanta, S. G. Bombardier, W. J. Cote, W. R. Hill, G. Kerszykowski, H. S. Landis, D. J. Poindexter, C. W. Pollard, G. H. Ross, J. G. Ryan, S. Wolff, J. E. Cronin, Proceedings of the VLSI Multilevel Interconnection Conference, pp. 144-152, June (1991).

${ }^{48}$ T. Licata, M. Okazaki, M. Ronay, S. Nguyen, H. Aochi, W. Landers, R. Filippi, D. Restaino, D. Knorr, J. G. Ryan, Proceedings of the VLSI Multilevel Interconnection Conference, pp. 596-602, June (1995).

${ }^{49}$ B. Vollmer, T. Licata, D. Restaino, J. G. Ryan, Thin Solid Films 247, 104 (1994).

${ }^{50}$ S. Lakshminarayanan, "Multilevel dual Damascene copper interconnections," Ph.D. Thesis, Rensselaer Polytechnic Institute, Feb. (1997).

${ }^{51}$ J. G. Ryan, J. E. Heidenreich, W. J. Cote, R. M. Geffken, T. N. Theis, Advanced Metallization and Interconnect Systems for ULSI Applications in 1997 Conference Proceedings, pp. 396, Oct. (1997).

52 The National Technology Roadmap for Semiconductors: Technology Needs. Semiconductor Industry Association, pp. 99, (1997).

${ }^{53}$ R. L. Jackson, E. Broadbent, T. Cacouris, A. Harrus, M. Biberger, E. Patton, T. Walsh, Solid State Technology 41(3), 49 (1998).

${ }^{54}$ C. Ryu, A. L. S. Loke, T. Nogami, S. S. Wong, International Reliability Physics Symposium Proceedings, pp. 201, May (1997). 
${ }^{55}$ S. S. Wong, C. Ryu, H. Lee, A. L. S. Loke, K.-W. Kwon, S. Bhattacharya, R. Eaton, R. Faust, B. Mikkola, J. Mucha, J. Ormando, Proceedings of the International Interconnect Technology Conference, pp. 107, June (1988).

${ }^{56}$ R. Venkatraman, A. Jain, J. Farkas, J. Mendonca, G. Hamilton, C. Capasso, D. Denning, C. Simpson, B. Rogers, L. Frisa, T. P. Ong, M. Herrick, V. Kaushik, R. Gregory, E. Apen, M. Angyal, S. Filipiak, P. Crabtree, T. Sparks, S. Anderson, D. Coronell, R. Islam, B. Smith, R. Fiordalice, H. Kawasaki, J. Klein, S. Venkatesan, E. Weitzman, MRS Symposium Proceedings 514, 41. (1998).

${ }^{57}$ S. Q. Wang, MRS Bulletin 19(8), 30 (1994).

${ }^{58}$ R. Venkatraman, A. Jain, J. Farkas, J. Mendonca, G. Hamilton, C. Capasso, D. Denning, C. Simpson, B. Rogers, L. Frisa, T. P. Ong, M. Herrick, V. Kaushik, R. Gregory, E. Apen, M. Angyal, S. Filipiak, P. Crabtree, T. Sparks, S. Anderson, D. Coronell, R. Islam, B. Smith, R. Fiordalice, H. Kawasaki, J. Klein, S. Venkatesan, E. Weitzman, MRS Symposium Proceedings 514, 41 (1998).

${ }^{59}$ R. L. Jackson, E. Broadbent, T. Cacouris, A. Harrus, M. Biberger, E. Patton, T. Walsh, Solid State Technology 41(3), 49 (1998).

${ }^{60}$ R. Venkatraman, A. Jain, J. Farkas, J. Mendonca, G. Hamilton, C. Capasso, D. Denning, C. Simpson, B. Rogers, L. Frisa, T. P. Ong, M. Herrick, V. Kaushik, R. Gregory, E. Apen, M. Angyal, S. Filipiak, P. Crabtree, T. Sparks, S. Anderson, D. Coronell, R. Islam, B. Smith, R. Fiordalice, H. Kawasaki, J. Klein, S. Venkatesan, E. Weitzman, MRS Symposium Proceedings 514, 41 (1998).

${ }^{61}$ K. Abe, Y. Harada, H. Onoda, Proceedings of the VLSI Multilevel Interconnection Conference, pp. 304, June (1995).

${ }^{62}$ D. S. Gardner, D. B. Fraser, Proceedings of the VLSI Multilevel Interconnection Conference, pp. 287, June (1995).

${ }^{63}$ J. A. T. Norman, B. A. Muratore, P. N. Dyer, D. A. Roberts, A. K. Hochberg, Proceedings of the VLSI Multilevel Interconnection Conference, pp. 123, June (1991).

${ }^{64}$ J. S. H. Cho, H.-K. Kang, S. S. Wong, Y. Shacham-Diamand, MRS Bulletin 18(6), 31 (1993).

${ }^{65}$ P. Singer, Semiconductor International 21(6), 90 (1998).

${ }^{66}$ D. Edelstein, J. Heidenreich, R. Goldblatt, W. Cote, C. Uzoh, N. Lustig, P. Roper, T. McDevitt, W. Motsiff, A. Simon, J. Dukovic, R. Wachnik, H. Rathore, R. Schulz, L. Su, S. Luce, J. Slattery, International Electron Device Meeting Technical Digest, pp. 773, Dec. (1997).

${ }^{67}$ T. Taylor, T. Ritzdorf, F. Lindberg, B. Carpenter, Solid State Technology 41(11), 47 (1998).

${ }^{68}$ V. M. Dubin, S. Lopatin, S. Chen, R. Cheung, C. Ryu, S. S. Wong, MRS Symposium Proceedings 514, 275 (1998).

${ }^{69}$ C. Ryu, K. W. Kwon, A. L. S. Loke, V. M. Dubin, R. A. Kavari, G. W. Ray, S. S. Wong, Symposium on VLSI Technology Technical Digest, pp. 156, June (1988).

${ }^{70}$ K. A. Perry, Symposium on VLSI Technology Technical Digest, pp. 25, June (1998).

${ }^{71}$ P. Singer, Semiconductor International 21(6), 90 (1998). 
${ }^{72}$ R. H. Dennard, F. H. Gaensslen, H.-N. Yu, V. L. Rideout, E. Bassous, A. R. LeBlanc, IEEE Journal of Solid-State Circuits SC-9(5), 256 (1974).

${ }^{73}$ K. C. Saraswat, F. Mohammadi, IEEE Transactions on Electron Devices ED-29(4), 645 (1982).

${ }^{74}$ M. T. Bohr, International Electron Device Meeting Technical Digest, pp. 2414, (1995).

${ }^{75}$ K. A. Monnig, SEMATECH Low Dielectric Constant Materials and interconnects Workshop Proceedings (San Diego, CA), 30 Apr. (1996).

${ }^{76}$ H. B. Bakoglu, J. D. Meindl, IEEE Transactions on Electron Devices ED-32(5), 903 (1985).

${ }^{77}$ H. B. Bakoglu, Circuits, Interconnections, and Packaging for VLSI . Reading, MA: Addison-Wesley, p. 194, (1990).

${ }^{78}$ D. R. Askeland, The Science and Engineering of Materials, 3rd edition. Boston, MA: PWS Publishing, pp. 603̈607, (1994).

${ }^{79}$ J. R. Black, International Reliability Physics Symposium Proceedings, pp. 148-159, 1967.

${ }^{80}$ D. S. Gardner, J. D. Meindl, K. C. Saraswat, IEEE Transactions on Electron Devices ED-34(3), 633 (1987).

${ }^{81}$ C. V. Thompson, J. R. Lloyd, MRS Bulletin 19, (1993).

${ }^{82}$ I. A. Blech, J. Appl. Phys., 47, 1203 (1976).

${ }^{83}$ S. Rhee, Y. Du, and P. S. Ho, "Thermal stress characteristics of Cu/oxide and Cu/low-k submicron interconnect structures," J. Appl. Phys., vol. 93, pp. 3926-33, 2003.

${ }^{84}$ R. S. Sprbello, "Microscopic driving forces for electronmigration," in Proc. Mater. Research Soc. Symp., vol. 427, 1996, pp. 73-81.

${ }^{85}$ M. Bohr, S. S. Ahmed, S. U. Ahmed, M. Bost, T. Ghani, J. Greason, R. Hainsey, C. Jan, P. Packan, S. Sivakumar, S. Thompson, J. Tsai, S. Yang, International Electron Device Meeting Technical Digest 847850 (1996).

${ }^{86}$ S.P. Jeng, R. H. Havemann, M.C. Chang, Advanced Metallization for Devices and Circuits - Science, Technology and Manufacturability Symposium, pp. 25, (1994).

${ }^{87}$ D. C. Thomas, S. S. Wong, IEEE Transactions on Electron Devices 39(4), 901 (1992).

${ }^{88}$ J. Ida, M. Yoshimaru, T. Usami, A. Ohtomo, K. Shimokawa, A. Kita, M. Ino, Symposium on VLSI Technology Technical Digest, pp. 59, June (1994).

${ }^{89}$ S. Y. Oh, K. J. Chang, IEEE Circuits and Devices 11(1), 16 (1995).

${ }^{90}$ S.P. Jeng, R. H. Havemann, M.C. Chang, Advanced Metallization for Devices and Circuits-Science, Technology and Manufacturability Symposium, pp. 46, (1994).

${ }^{91}$ M. Hussein, Intel Corporation, quarterly presentation, Hillsboro, OR (2002)

${ }^{92}$ D. H. Cho, Y. S. Eo, M. H. Seung, N. H. Kim, J. K. Wee, O. K. Kwon, H. S. Park, International Electron Device Meeting Technical Digest 619 (1996).

${ }^{93}$ D. C. Thomas, S. S. Wong, IEEE Transactions on Electron Devices 39(4), 901 (1992).

${ }^{94}$ R. DeJule, Semiconductor International, June (2000). 
${ }^{95}$ D. Dornisch, G. Li, M. Brongo, Solid State Technology, 43(5), (2000).

${ }^{96}$ D. Dornisch, G. Li, M. Brongo, Solid State Technology, 43(5), (2000).

${ }^{97}$ W. M. Lee, U.S. patent 4,824,763 (1989).

${ }^{98}$ B. Kanegsberg, ed. Handbook for Critical Cleaning. CRC Press: New York (2001).

${ }^{99}$ D. Louis, presented at Semicon Europa, Munich, Germany, April (2001).

${ }^{100}$ N. G. Mistkawi, M. A. Hussein, M. Ziomek-Moroz, S. B. Rananavare, $J$. Electrochem. Soc. 157, C24 (2010).

${ }^{101}$ G. S. Oehrlein, G. J. Scilla, S. Jeng, Appl. Phys, Lett. 52, 907 (1988).

${ }^{102}$ G. S. Oehrlein, Y.H. Lee, J. Vac.Sci.Technol. A4, 1585 (1987).

${ }^{103}$ D. K. Schroder, Materials and Device Characterization, p.576, John Wiley \& Sons, Inc., New York (1998).

104 A. Somashekhar, H.Ying, P.B. Smith, D.B. Aldrich, R.J. Nemanich, J. Electrochem. Society 146, 2318 (1999).

${ }^{105}$ Q. Han, B.White, I.L.Berry, C. Waldfried, O.Escorcia, Solid State Phenomena 103104, 341 (2005).

${ }^{106}$ K. Ueno, V. M. Donnelly, T. Kikkawa, J. Electrochem.Soc. 144, 2565, (1997).

${ }^{107}$ T. H. Park, Y. S. Ko, T. E. Shim, J. G. Lee, Y. K. Kim, J. Electrochem. Soc. 142(2), 571 (1995).

${ }^{108}$ W. A. Wojtczak, G. Guan, D.N. Fine, S.A. Fine, U.S. Pat. No. 6224785.

${ }^{109}$ W. Kern, J. Electrochem. Soc. 137(6), 1890 (1990).

${ }^{110}$ M. Hussein, J. He, IEEE Transactions on Semiconductor Manufacturing 18(1), 69 (2005).

${ }_{111}^{11}$ J. S. Kim, H. Morita, J. D. Joo, T. Ohmi, J. Electrochem. Soc. 144, 3275 (1997).

${ }^{112}$ G. J. Norga, M. Platero, K. A. Black, A. J. Reddy, J. Michel, L. C. Kimerling, J. Electrochem. Soc., 144, 2801 (1997).

${ }^{113}$ L. Mouche, F. Tardif, J. Derrien, J. Electrochem. Soc. 142, 2395 (1995).

${ }^{114}$ G. Li, E.A. Kneer, B. Vermeire, H.G. Parks, S. Raghavan, J. Electrochem. Soc. (145), 241 (1998).

${ }^{115}$ N. G. Mistkawi, M. A. Hussein, Intel Corporation research archive, Hillsboro OR. (2002)

${ }^{116}$ T. Ohmi, T. Imaoka, T. Kezuka, J. Takano, M. Kogure. J. Electrochem. Soc. 140(3), 811 (1993).

${ }^{117}$ W. Kern, D. A. Puotien, RCA Rev. 31, 187 (1970).

${ }^{118}$ W. Kern. J. Electrochem. Soc. 137(6), 1887 (1990).

${ }^{119}$ T. H. Park, Y-S. Ko, T.-E. Shim, J-G Lee. J. Electrochem. Soc. 142(2), 3173 (1995).

${ }^{120}$ T. Shimono, M. Tsuji Proc. Second Symp. On Defects in Silicon. Defects in Silicon II W. M. Bullis, U. Gösele Editors, J Electrochem. Soc., 22, 361 (1991).

${ }^{121}$ T. Ohmi, T. Imaoka, I. Sugiyama, T. Kesuka J. Electrochem. Soc. 139, 811 (1992).

${ }^{122}$ V. Bertagnay, F. Rouellez M. Chemlaz., Semicond. Sci. Technol. 13, 444 (1998).

${ }^{123}$ M.B. Beaver, Encyclopedia of Material Science and Engineering, Pergamon Press, 841-88 (1986), 
${ }^{124}$ Kirk-Othmer Encyclopedia of Chemical Technology, 3rd ed., Vol. 6, p. 819, John Wiley \& Sons, Inc., (1979).

${ }^{125}$ M.B. Beaver, Encyclopedia of Material Science and Engineering, p.841, Pergamon Press, (1986).

${ }^{126}$ Pourbaix, M., Atlas of Electrochemical Equilibria in Aqueous Solutions, p. 244, Houston, Tex., NACE International, (1974).

${ }^{127}$ D. A. Jones, "Principles and Prevention of Corrosion", Second Edition, p. 50, Prentice-Hall, Inc. (1996).

${ }^{128}$ U. R. Evans, Corrosion and Corrosion Control, An Introduction to Metallic corrosion, 3rd ed., p. 60, John Willy \& Sons, Inc., (1985).

${ }^{129}$ B. D. Craig, Fundamental Aspects of Corrosion Films in Corrosion Science, P. 9, Plenum Press (1991).

${ }^{130}$ R. T. DeHoff, “Thermodynamics in Materials Science”, P.89, McGraw-Hill, NY (1993).

${ }^{131}$ M. Pourbaix: "Atlas of Electrochemical Equilibria in Aqueous Solutions", Pergamon, New York, (1966).

132 E.D. Verink: "Simplified Procedure for Constructing Pourbaix Diagrams", in Uhlig's Corrosion Handbook 2nd Edition, Ed. R.W. Revie, Jon Wiley, New York, (2000).

${ }^{133}$ D. A. Jones, "Principles and Prevention of Corrosion", p. 55-65, 79-101, 115-139, Macmillan Publishing Co, (1996).

${ }^{134}$ Kirk-Othmer, Encyclopedia of Chemical Technology, 3rd ed., Vol. 6, p. 819, John Wiley \& Sons, Inc., (1979).

${ }^{135}$ P. Singer, Semiconductor International 21(6), 90 (1998).

${ }^{136}$ D. Edelstein, J. Heidenreich, R. Goldblatt, W. Cote, C. Uzoh, N. Lustig, P. Roper, T. McDevitt, W. Motsiff, A. Simon, J. Dukovic, R. Wachnik, H. Rathore, R. Schulz, L. $\mathrm{Su}$, S. Luce, J. Slattery, p. 773, International Electron Device Meeting Technical Digest, ( 1997).

${ }^{137}$ See for example, Denny A. Jones in "Principles and Prevention of Corrosion", figure 4.5 page 119, New York, 1 ed. McMillan Pub, New York 1991.

${ }^{138}$ M. Pourbaix, Atlas of Electrochemical Equilibria in Aqueous Solutions, NACE International, Houston, Tx., pp (1974).

${ }^{139}$ M. V. Vazquez, S.R. Sanchez, E. J. Calvo, and D. J. Schiffrin, J. Electroanal. chem. ., 374, 179 (994).

${ }^{140}$ J. S. Jeon, S. Raghavan, H. G. Parks, J. K. Lowell, and L. Ali, J. Electrochem. Soc., 143, 2870 (1996).

141 K.K. Yoneshige, H.G. Parks, S. Raghaven, J.B. Hiskey, P.J. Resnick, J. Electrochem. Soc. 142, 671 (1995).

${ }^{142}$ W. Kern, RCA Rev., Part I, 31, 207 (1970); Part II, 31, 234 (1970); Part III, 32, 64 (1971).

${ }^{143}$ K. K. Yoneshige, H. G. Parks, S. Ragaavan, J. B. Hiskey, P. J. Resnick, J. Electrochem. Soc. 142, 671 (1995). 
144 J. S. Jeon, S. Raghavan, H. G. Parks, J. K. Lowell, L. Ali, J. Electrochem. Soc. 143, 2870 (1996).

${ }^{145}$ H. Morinaga, M. Suyama, M. Nose, S. Verhaverbeke, T. Ohmi, IEICE Trans. Electron E79-C, 343 (1996).

${ }^{146}$ T. Homma, C. P. Wade, C. E. D. Chidsey, J. Phys. Chem. B 102, 7919 (1998).

${ }^{147}$ G. Li, E. A. Kneer, B. Vermerire, H. G. Parks, S. Raghavan, J. S. Jeon, J. Electrochem. Soc. 145, 241 (1998).

${ }^{148}$ X. Cheng, G. Li, E. A. Kneer, B. Vermeire, H. G. Parks, S. Raghavan, J. S. Jeon, J. Electrochem. Soc. 145, 247 (1998).

${ }^{149}$ E. Hsu, H. G. Parks, R. Craigin, J. Electrochem. Soc. 139(12), 3659 (1992).

${ }^{150}$ G. Li, E. A. Kneer, B. Vermeire, H. Parks, S. Raghavan, J. Electrochem. Soc. 145 (1), 352 (1998).

${ }^{151}$ O. J. Anttila 1, M. V. Tilli, M. Schaekers, C. L. Claeys. J. Electrochem. Soc. 139(4), 1180 (1992).

${ }^{152}$ X. Cheng, C. Gu, Z-D Feng, Journal of The Electrochemical Society 150(2), 112 (2003).

153 J. Norga, M. Platero, K. A. Black, A. J. Reddy, J. Michel, L C. Kimerling, J. Electrochem. Soc. 144(8), 2801 (1997)

${ }^{154}$ E. D'Elia, O. E. Barcia, O. R. Mattos, N. Pébere, B. Tribollet, J. Electrochem. Soc. 143, 961 (1996)

${ }^{155}$ V. Bertagna, F. Rouelle, G. Revel, M. Chemla, J. Electrochem. Soc. 143(1), 3532 (1996).

${ }^{156}$ O. M. R. Chyan, J. J. Chen, H. Y. Chien, J. Sees, L. Hall. J. Electrochem. Soc. 143, 92 (1996).

${ }^{157}$ D. Ernur, V. Terzieva, W. Wu, S. H. Brongersma, K. Maex, J. Electrochem. Soc. 151(12), B636, (2004).

${ }^{158}$ D. Ernur, L. Carbonell, K. Maex, Materials Research Society Spring Meeting, Symposium Z, Online Proceedings (2003).

${ }^{159}$ P. R. Besser, E. Zschech, W. Blum, D. Winter, R. Ortega, S. Rose, M. Herrick, M. Gall, S. Thrasher, M. Tiner, B. Baker, G. Braeckelmann, L. Zhao, C. Simpson, C. Capasso, H. Kawasaki, E. Weitzman. J. Electron. Mater. 30, 320 (2001).

${ }^{160}$ H. Y. H. Chan, C. G. Takoudis and M. J. Weaver, The Journal of Physical Chemistry B, 103, 357 (1998).

${ }^{161}$ D. B. Hibbert, S. Richards and V. Gonzalves, Corrosion Science, 30, 367 (1990).

${ }^{162}$ E. D'Elia, O. E. Barcia, O. R. Mattos, N. Pebere and B. Tribollet, Journal of The Electrochemical Society, 143, 961 (1996).

163 A. G. Zelinsky, B. Y. Pirogov and O. A. Yurjev, Corrosion Science, 46, 1083 (2004).

${ }^{164}$ Z. Zembura and J. Bugajski, Corrosion Science, 21, 69 (1981).

${ }^{165}$ M. A. Hussein, J. He. IEEE Transactions on Semiconductor Manufacturing. 1(18), 69 (2005).

${ }^{166}$ M. Hiroi, Proc. IITC 2001 Piscataway, NJ, 10295 (2001). 
${ }^{167}$ P. A. Kohl, D. M. Bhusari, M.Wedlake, C. Case, F. P. Klemens, J. Miner, L. Byung-Chan, R. J. Gutmann, R. Shick, Electron Device Lett. 21(12), 557 (2000).

${ }^{168}$ J. J. Waeterloos, E. O. Shaffer II, T. Stokich Jr., J. Hetzner, D. Price, L. Booms, R. A. Donaton, G. Beyer, B. Coenegrachts, R. Caluwaerts, H. Struyf, Z. S. Tokei, I. Vervoort, B. Sijmus, I. Vos, K. Maex, T. Komiya, M. Iwashita, Proc. IEEE Int. Interconnect Technol. Conf., Jun. 4-6, 60 (2001)

${ }^{169}$ M. Hussein, R. Brain, R. Turkot, J. Leu, V. Singh, S. Sivakumar, in Proc. Int. Interconnect Technology Conf. Piscataway, NJ, Jun. 2-4, 33 (2003).

${ }^{170}$ R. Kammura. proceedings of VLSI Technology. 107 (2003).

${ }^{171}$ H. Miyajima, Proc. of IEDM. P. 329 (2004).

172 N. Nakamura, Proc. Of IEEE, IITC. p. 228 (2004).

${ }^{173}$ M. A. Hussein, J. He. IEEE Transactions on Semiconductor Manufacturing. 1(18), 69 (2005)

${ }^{174}$ E. Braun, Semicond. Int. p. 158 (2004).

${ }^{175}$ W. Zhang, S. H. Brongersma, T. Clarysse, Proc. Of IEEE, IITC Conf., p. 30 (2005).

${ }^{176}$ A. Kajita, T. Usui, M. Yamada, E. Ogawa, T. Katata, A. Sakata, H. Miyajima, A. Kojima, R. Kanamura, Y. Ohoka, H. Kawashima, K. Tabuchi, K. Nagahata, Y. Kato, T. Hayashi, S. Kadomura, H. Shibata, in Proc. Int. Interconnect Technol. Conf. Piscataway, NJ, Jun. 2-4, 9 (2003).

${ }^{177}$ M. Assous, J. Simon, L. Broussous, C. Bourlot, M. Fayolle, O. Louveau, A. Roman, E. Tabouret, H. Feldis, D. Louis, J. Torres, in Proc. Int. Interconnect Technology Conf. Piscataway, NJ, Jun. 2-4, 97 (2003).

${ }^{178}$ T. Furusawa, S. Machida, D. Ryuzki, K. Sameshima, T. Ishida, K. Ishikawa, N. Miura, N. Konishi, T. Saito, H. Yamaguchi, in Proc. Int. Interconnect Technology Conf. Piscataway, NJ, Jun. 2-4, 195 (2003).

${ }^{179}$ S.Wolf, R. N. Tauber, Silicon Processing for the VLSI Era. Sunset Beach, CA: Lattice, 1 (1986).

180 W. R. Runyan, K. E. Bean, Semiconductor Integrated Circuit Processing Technology. Reading, MA: Addison-Wesley, (1990).

181 J. L. Vossen, W. Kern, Eds., Thin Film Processes. New York, NY: Academic, 1 (1978).

${ }^{182}$ P. Walker, W. H. Tarn, CRC Handbook of Metal Etchants, CRC, Boca Raton, FL (1991).

${ }^{183}$ D. R. Lide, ed., CRC Handbook of Chemistry and Physics, Internet Version 2005, <http://www.hbcpnetbase.com>, CRC Press, Boca Raton, FL, 2005.

${ }^{184}$ P. Lagasse, Columbia Encyclopedia (6th edition). New York: Columbia University 322 (1993).

${ }^{185}$ G. Gleig, Encyclopæedia Britannica, Encyclopædia Britannica (2006).

${ }^{186}$ N. Casillas, S. Charlebois, W. H. Smyrl, H. S. White, J. Electrochem. Soc. 141(3), 636 (1994).

${ }^{187}$ A. A. Stwertka, Guide to the elements. 2nd ed. New York: Oxford University Press, 245 (2002). 
${ }^{188}$ E. Lassner, W. D. Schubert, Tungsten: Properties, Chemistry, Technology of the Element, Alloys, and Chemical Compounds, 179 (1998).

189 J. E. Emsley, The elements. 2nd ed. New York: Oxford University Press, 56 (1991).

190 J. E. Emsley, The elements. 2nd ed. New York: Oxford University Press, 89 (1991).

${ }^{191}$ C. J. Brinker, G. W. Scherer, Sol-Gel Science: The Physics and Chemistry of SolGel Processing, Academic Press, Inc., New York, p. 189 (1990).

${ }^{192}$ T. W. Zerda, I. Artaki, J. Jonas, J. Non-Crystalline Solids 81, 377 (1986).

${ }^{193}$ E. Colvin, Silicon in Organic Synthesis Butterworth: London, 194 (1981).

${ }^{194}$ N. Auner, J. Weis, Organosilicon Chemistry VI: From Molecules to Materials, John Wiley \& Sons, Inc., New York, 6, 287 (2005).

${ }^{195}$ R. Aelion, A. Loebel, F. J. Eirich, Am. Chem. Soc. 72, 5705 (1950).

196 T. N. M. Bernards, M. J. Van Bommel, J. A. Jansen, Journal of Sol-Gel Science and Technology 13, 749 (1998).

${ }^{197}$ L. L. Hench, J. K. West, Chem. Rev. 90, 35 (1990)

${ }^{198}$ O. Lev, Analytical Chemistry 67(1), 22A (1995).

${ }^{199}$ C. J. Brinker and G. W. Scherer, Sol-Gel Science: The Physics and Chemistry of Sol-Gel Processing, Academic Press, Inc., New York, p. 338 (1990).

${ }^{200}$ C. J. Brinker, G. W. J. Scherer, Non-Crystalline Solids 70, 301 (1985).

${ }^{201}$ K. D. Keefer, in: Silicon Based Polymer Science: A Comprehensive Resource; eds. J.M. Zeigler and F.W.G. Fearon, ACS Advances in Chemistry Ser. No. 224, (American Chemical Society: Washington, DC, p. 227 (1990).

${ }^{202}$ C. J. Brinker, G. W. Scherer, Sol-Gel Science: The Physics and Chemistry of SolGel Processing, Academic Press, Inc., New York, p. 239 (1990).

${ }^{203}$ L. L.Hench, J. K. West, Chem. Rev. 90, 35 (1990).

${ }^{204}$ M. Prassas, L. L. Hench, Ultrastructure Processing of Ceramics, Glasses, and Composites, John Wiley \& Sons, New York, 125, p. 100, (1984).

${ }^{205}$ W. Ströber, A. Fink, and E. Bohn, J. Colloid Interface. Sci. 26, 62 (1968).

${ }^{206}$ T. W. Zerda, I. Artaki, J. Jonas, J. Non-Crystalline Solids 81, 374 (1986).

${ }^{207}$ R. A. Assink, B. D. Kay, J. Non-Crystalline Solids 107, 35 (1998).

${ }^{208}$ C. J. Brinker, J. Non-Crystalline Solids 48, 47 (1982).

${ }^{209}$ C. J. Brinker, J. Non-Cryst. Solids 100, 30 (1988).

${ }^{210}$ Q. Huo, Nature 368, 317 (1994).

211 J-M. Kim, Chem. Commun. 437 (2000).

212 W. Qafsaoui, Ch. Blanc, N. Pe'be`re, A. Srhiri, G. Mankowski, J. Appl. Electrochem. 30, 959 (2000).

${ }^{213}$ V. Brusic, M. A. Frisch, B. N. Eldridge, F. P. Novak, F. B. Kaufman, B. M. Rush, G. S. Frankel, J. Electrochem. Soc. 138, 2253 (1991).

214 J. B. Cotton and I. R. Scholes, Br. Corros. J., London, 2,1 (1967).

${ }^{215}$ G. W. Poling, Corros. Sci., 10, 359 (1970).

216 T. Notoya, G. W. Poling, Corrosion (Houston), 32, 216 (1976).

${ }^{217}$ M. Metikoš-Huković, R. Babić, I. Paić, J. Appl. Electrochem., 30, 617 (2000).

${ }^{218}$ D. Tromans, J. Electrochem. Soc., 145, L42 (1998).

${ }^{219}$ Y, Jiang, J. B. Adams, J. of Surface Science 529, 428 (2003) 
${ }^{220}$ J. Marsh, R. Pearson, B. Strickland, J. Moore, S. Raghavan, Semiconductor Pure Water and Chemicals Conference., (2005).

${ }^{221}$ R. S. Lillard, J. R. Scully, J. Electrochem. Soc. 145, 2718 (1998).

${ }^{222}$ D. V. Tzaneva, V. I. Dimitrova, P. E. Hovsepyan, Thin Solid Films 295, 178 (1997).

${ }^{223}$ D. J. Monk, D. S. Soane, R. T. Howe, J. Electrochem. Soc., 140 (8), 2339 (1993) and the references cited there in.

${ }^{224}$ W. F. Finney, E. Wilson, A. Callender, M. D. Morris, L. W. Beck, Environ. Sci. Technol., , 40 (8), 2572 (2006).

${ }^{225}$ D. J. Monk, D. S. Soane, R. T. Howe, J. Electrochem. Soc., 140 (8), 2339 (1993) and the references cited there in.

${ }^{226}$ W. F. Finney, E. Wilson, A. Callender, M. D. Morris, L. W. Beck, Environ. Sci. Technol., , 40 (8), 2572 (2006). 


\section{Appendix A}

\section{Kinetic Analysis for Copper Dissolution}

$$
\begin{aligned}
& O_{2}^{s \ln } € \quad O_{2}^{a d s} k_{0}^{e q}=\frac{\left[O_{2}^{a d s}\right]}{\left[O_{2}^{s \ln }\right]} \\
& O_{2}^{a d s} \stackrel{k_{1}}{\longrightarrow} 2 O^{a d s} \frac{d\left[O^{a d s}\right]}{d t}=2 k_{1}\left[O_{2}^{a d s}\right]+k_{-2}\left[C u_{2}(O H)_{2}\right]-k_{2}\left[O^{a d s}\right]=0 \\
& 2 \mathrm{Cu}+\mathrm{O}^{a d s}+\underset{k_{-2}}{\mathrm{H}_{2} \mathrm{O} €^{k_{2}}} \mathrm{Cu}_{2}(\mathrm{OH})_{2} \quad k_{2}^{e q}=\frac{\left[\mathrm{Cu}_{2}(\mathrm{OH})_{2}\right]}{\left[\mathrm{O}^{a d s}\right]}=\frac{k_{2}\left[\mathrm{Cu}_{2}(\mathrm{OH})_{2}\right]}{2 k_{1}\left[\mathrm{O}_{2}^{a d s}\right]+k_{-2}\left[\mathrm{Cu}_{2}(\mathrm{OH})_{2}\right]} \\
& =\frac{k_{2}\left[\mathrm{Cu}_{2}(\mathrm{OH})_{2}\right]}{2 k_{1} k_{0}^{e q}\left[\mathrm{O}_{2}^{s \ln }\right]+k_{-2}\left[\mathrm{Cu}_{2}(\mathrm{OH})_{2}\right]} \\
& \therefore\left[\mathrm{Cu}_{2}(\mathrm{OH})_{2}\right]=\frac{2 k_{1} k_{0}^{e q}\left[\mathrm{O}_{2}^{s \ln }\right]}{\left(k_{2} / k_{2}^{e q}-k_{-2}\right)}=k^{\prime}\left[\mathrm{O}_{2}^{s \ln }\right] \\
& \mathrm{Cu}_{2}(\mathrm{OH})_{2}+H F \stackrel{k_{3}}{\longrightarrow} \mathrm{Cu}(\mathrm{OH}) \mathrm{CuF}+\mathrm{H}_{2} \mathrm{O} \quad \frac{d[\mathrm{Cu}(\mathrm{OH}) \mathrm{CuF}]}{d t}=k_{3}\left[\mathrm{Cu}_{2}(\mathrm{OH})_{2}\right][H F]-k_{4}[\mathrm{Cu}(\mathrm{OH}) \mathrm{CuF}][\mathrm{HF}]=0 \\
& \mathrm{Cu}(\mathrm{OH}) \mathrm{CuF}+\mathrm{HF} \stackrel{k_{4}}{\longrightarrow} \mathrm{Cu}_{2} F_{2}+\mathrm{H}_{2} \mathrm{O} \frac{d\left[\mathrm{Cu}_{2} F_{2}\right]}{d t}=k_{4}[\mathrm{Gu}(\mathrm{OH}) \mathrm{CuF}][H F]-k_{5}\left[\mathrm{Cu}_{2} F_{2}\right]=0 \\
& \mathrm{Cu}_{2} F_{2} \stackrel{k_{5}}{\longrightarrow} \mathrm{Cu}^{0}+\mathrm{CuF}_{2} \quad \frac{d\left[\mathrm{Cu}^{+2}\right]}{d t}=k_{5}\left[\mathrm{Cu}_{2} F_{2}\right]=k_{4}[\mathrm{Cu}(\mathrm{OH}) \mathrm{CuF}][\mathrm{HF}]=k_{3}\left[\mathrm{Cu}_{2}(\mathrm{OH})_{2}\right][H F]
\end{aligned}
$$

Substituting for $\mathrm{Cu}_{2}(\mathrm{OH})_{2}$ concentration from the reactions:

$$
\frac{d\left[C u^{+2}\right]}{d t}=k_{3}\left[C u_{2}(O H)_{2}\right][H F]=\frac{2 k_{1} k_{3} k_{0}^{e q}\left[O_{2}^{s \ln }\right][H F]}{\left(k_{2} / k_{2}^{e q}-k_{-2}\right)}=\frac{2 k_{0}^{e q} k_{1} k_{2}^{e q} k_{3}\left[O_{2}^{s \ln }\right][H F]}{\left(k_{2}-k_{-2} k_{2}^{e q}\right)}
$$

The rate law for copper dissolution predicts a first order kinetics with respect to $\mathrm{HF}$ and dissolved oxygen, consistent with experimental data. 


\section{Appendix B}

\section{DOE Fitted Model Analysis Outcome for $\mathrm{Ti}, \mathrm{SiO}_{\mathrm{x}}$, and $\mathrm{Si}_{3} \mathrm{~N}_{4}$ Etch Rates}

Figures aB.1, aB.3, and aB.5 show plots of actual (observed) vs. predicted values for each response variable, $\mathrm{Ti}, \mathrm{SiO}_{\mathrm{x}}$, and $\mathrm{Si}_{3} \mathrm{~N}_{4}$ etch rates respectively, which gives a visual assessment of how well the fitted models predict the response variables.

The etch rate summary of fit and analysis of variance for the fitted model are shown in Tables aB.1, aB.2, and aB.3 for Ti, $\mathrm{SiO}_{x}$, and $\mathrm{Si}_{3} \mathrm{~N}_{4}$ etch rates respectively. The Ti etch rate parameter estimates, effect tests, and sorted parameters for the fitted model are illustrated in Tables aB.2, aB.4, and aB.6 for $\mathrm{Ti}, \mathrm{SiO}_{\mathrm{x}}$, and $\mathrm{Si}_{3} \mathrm{~N}_{4}$ etch rates respectively. The effects estimates are herein compared and a Pareto plot is constructed. Pareto plots are useful graphical method for comparing effects estimates of the absolute values of all effects. It is clear that HF, MTES, and the HF/MTES ratio have significant effect on etch rate.

\section{aB-1 Ti Etch Rate Analysis}

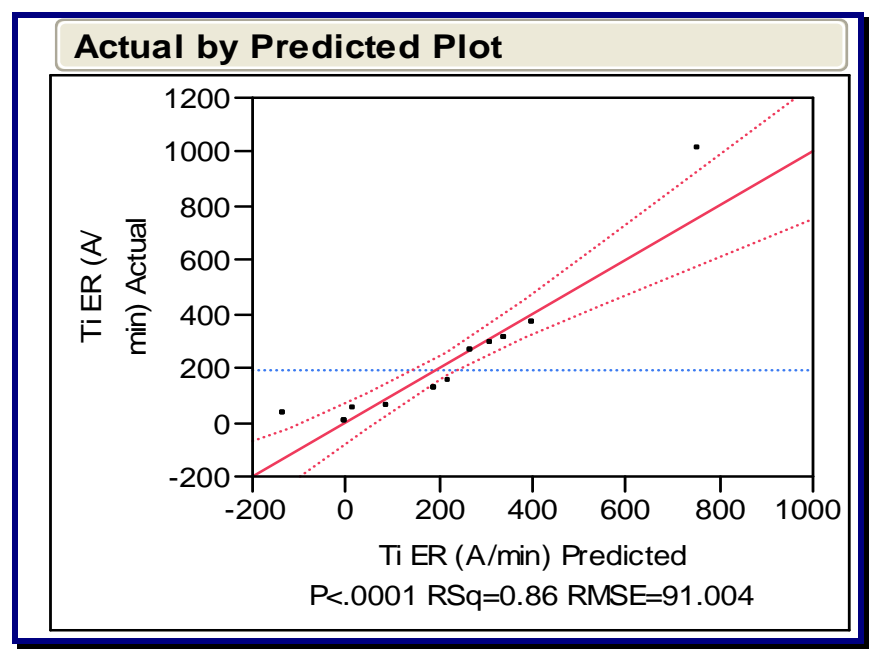

Figure aB.1 Ti etch rate of observed vs. predicted from the fitted model. 
Table aB.1 Ti etch rate summary of fit and analysis of variance for the fitted model.

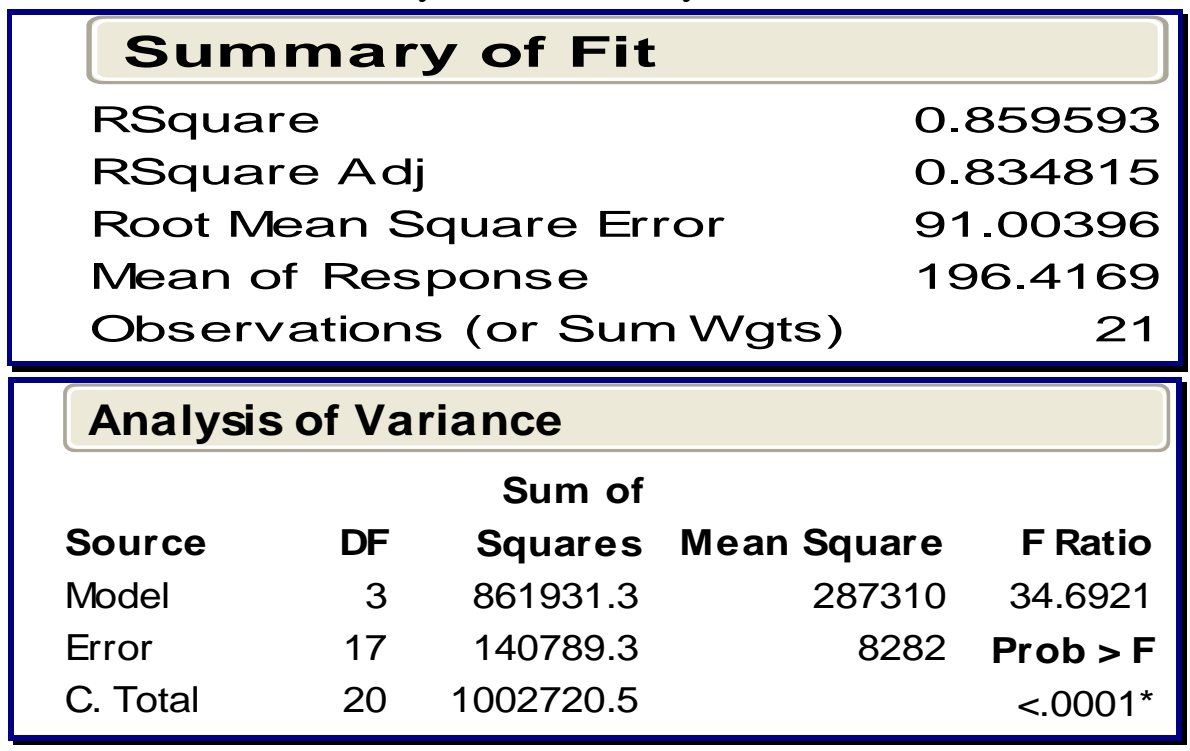

Table aB.2 Ti etch rate parameter estimates, effect tests, and sorted parameters for the fitted model.

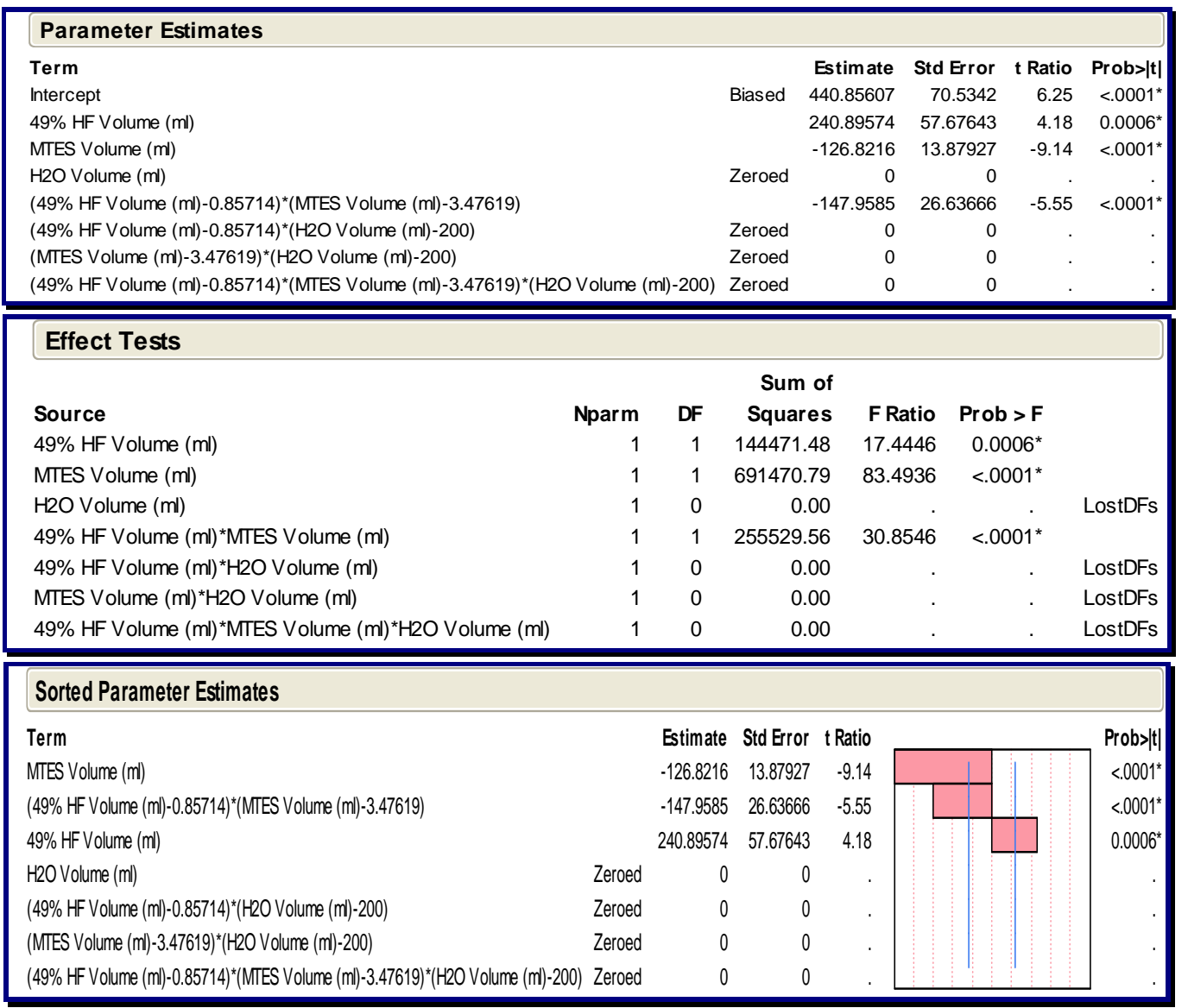


aB-2 Silicon Oxide Etch Rate Analysis

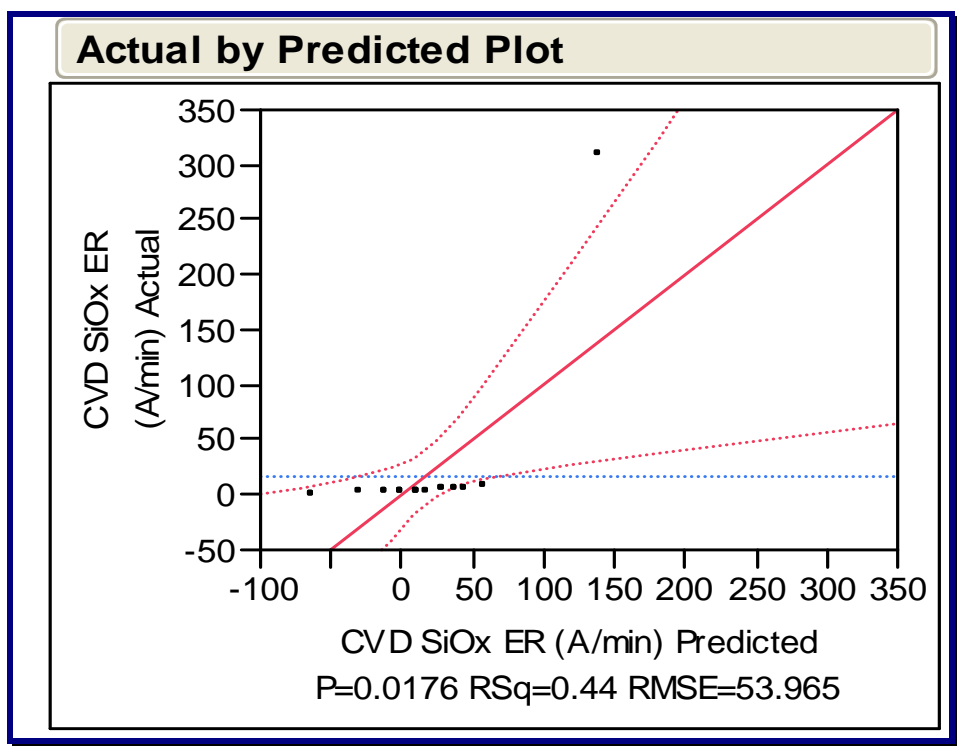

Figure aB.2 $\mathrm{SiO}_{\mathrm{x}}$ etch rates of observed vs. predicted from the fitted model.

Table aB.3 $\mathrm{SiO}_{\mathrm{x}}$ etch rate summary of fit and analysis of variance for the fitted model.

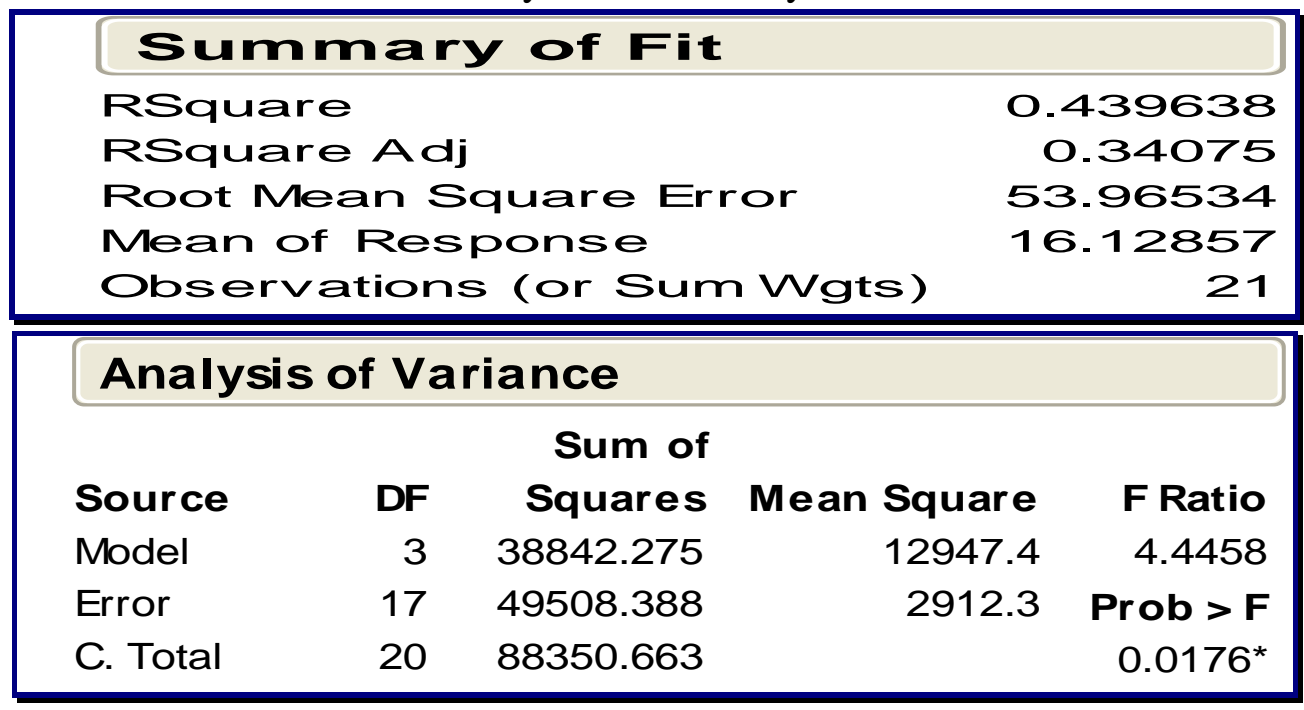


Table aB.4 $\mathrm{SiO}_{x}$ etch rate parameter estimates, effect tests, and sorted parameters for the fitted model.

\begin{tabular}{|c|c|c|c|c|c|c|c|c|}
\hline \multicolumn{9}{|l|}{ Parameter Estimates } \\
\hline \multicolumn{3}{|l|}{ Term } & \multicolumn{3}{|c|}{ Estimate } & Std Error & \multirow{2}{*}{$\begin{array}{r}\text { t Ratio } \\
2.41\end{array}$} & Prob $>\mid t$ \\
\hline Intercept & & & Biased & \multicolumn{2}{|c|}{100.70719} & 41.82677 & & $0.0277^{\star}$ \\
\hline $49 \%$ HF Volume (ml) & & & & \multicolumn{2}{|c|}{21.499126} & 34.20212 & \multirow{2}{*}{$\begin{array}{r}0.63 \\
-3.52\end{array}$} & \multirow{2}{*}{$\begin{array}{l}0.5380 \\
0.0026\end{array}$} \\
\hline MTES Volume (ml) & & & & \multicolumn{2}{|c|}{-28.97056} & 8.230406 & & \\
\hline H2O Volume (ml) & & & Zeroed & & 0 & 0 & . & . \\
\hline (49\% HF Volume $(\mathrm{mll})-0.85714)^{*}($ MTES Volume $(\mathrm{mll})-3.47619)$ & & & & \multicolumn{2}{|c|}{-33.79899} & 15.79554 & -2.14 & $0.0472^{*}$ \\
\hline$(49 \% \text { HF Volume }(\mathrm{ml})-0.85714)^{*}(\mathrm{H} 2 \mathrm{O}$ Volume $(\mathrm{mll})-200)$ & & & Zeroed & \multicolumn{2}{|c|}{0} & 0 & & . \\
\hline (MTES Volume (ml)-3.47619)*(H2O Volume (ml)-200) & & & Zeroed & \multicolumn{2}{|r|}{0} & 0 & & . \\
\hline$(49 \% \text { HF Volume }(\mathrm{ml})-0.85714)^{\star}(\text { MTES Volume }(\mathrm{ml})-3.47619)^{\star}(\mathrm{H} 2 \mathrm{O}$ & O Volume & nl)-200) & Zeroed & \multicolumn{2}{|r|}{0} & 0 & . & . \\
\hline \multicolumn{9}{|l|}{ Effect Tests } \\
\hline \multicolumn{9}{|c|}{ Sum of } \\
\hline $49 \%$ HF Volume (ml) & 1 & 1 & 1150.708 & & 0.3951 & & 380 & \multirow{4}{*}{ LostDFs } \\
\hline MTES Volume (ml) & 1 & 1 & 36082.879 & & 12.3900 & & $026^{*}$ & \\
\hline H2O Volume (ml) & 1 & 0 & \multicolumn{2}{|c|}{0.000} & & & & \\
\hline 49\% HF Volume (ml)*MTES Volume (ml) & 1 & 1 & 13334.247 & & 4.5787 & & $472^{*}$ & \\
\hline 49\% HF Volume (ml)*H2O Volume (ml) & 1 & 0 & 0.000 & & & . & . & LostDFs \\
\hline MTES Volume (ml)*H2O Volume (ml) & 1 & 0 & 0.000 & & & . & . & LostDFs \\
\hline 49\% HF Volume $(\mathrm{ml})^{*}$ MTES Volume $(\mathrm{ml})^{*} \mathrm{H} 2 \mathrm{O}$ Volume $(\mathrm{ml})$ & 1 & 0 & 0.000 & & & . & . & LostDFs \\
\hline Sorted Parameter Estimates & & & & & & & & \\
\hline Term & & Estimate & Std Error & t Ratio & & & & Prob $>|t|$ \\
\hline MTES Volume (m) & & -28.97056 & 68.230406 & -3.52 & & & & $0.0026^{*}$ \\
\hline (49\% HF Volume (m)-0.85714)*(MTES Volume (m))-3.47619) & & -33.79899 & 915.79554 & -2.14 & & & & $0.0472^{*}$ \\
\hline $49 \%$ HF Volume (m) & & 21.499126 & $6 \quad 34.20212$ & 0.63 & & & & 0.5380 \\
\hline H2O Volume (ml) & Zeroed & 0 & $0 \quad 0$ & . & & & & \\
\hline (49\% HFV Volume (m))-0.85714) $)^{*}($ H2O Volume (m))-200) & Zeroed & 0 & 0 & . & & & & \\
\hline (MTES Volume (m)-3.47619)*(H2O Volume (m)-200) & Zeroed & 0 & 0 & . & & & & \\
\hline$(49 \%$ HF Volume $(\mathrm{m}))-0.85714)^{*}(\text { MTES Volume }(\mathrm{m})-3.47619)^{*}(\mathrm{H} 20$ Volume $(\mathrm{m})-20$ & 00) Zeroed & 0 & 0 & . & & & & \\
\hline
\end{tabular}

\section{aB-3 Silicon Nitride Etch Rate Analysis}

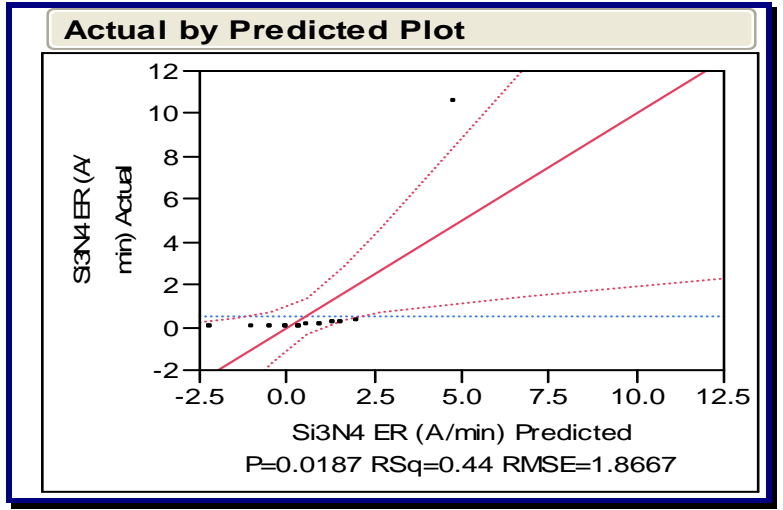

Figure aB.3 $\mathrm{Si}_{3} \mathbf{N}_{4}$ etch rate of observed vs. predicted from the fitted model. 
Table aB.5 $\mathbf{S i}_{3} \mathbf{N}_{4}$ etch rate summary of fit and analysis of variance for the fitted model.

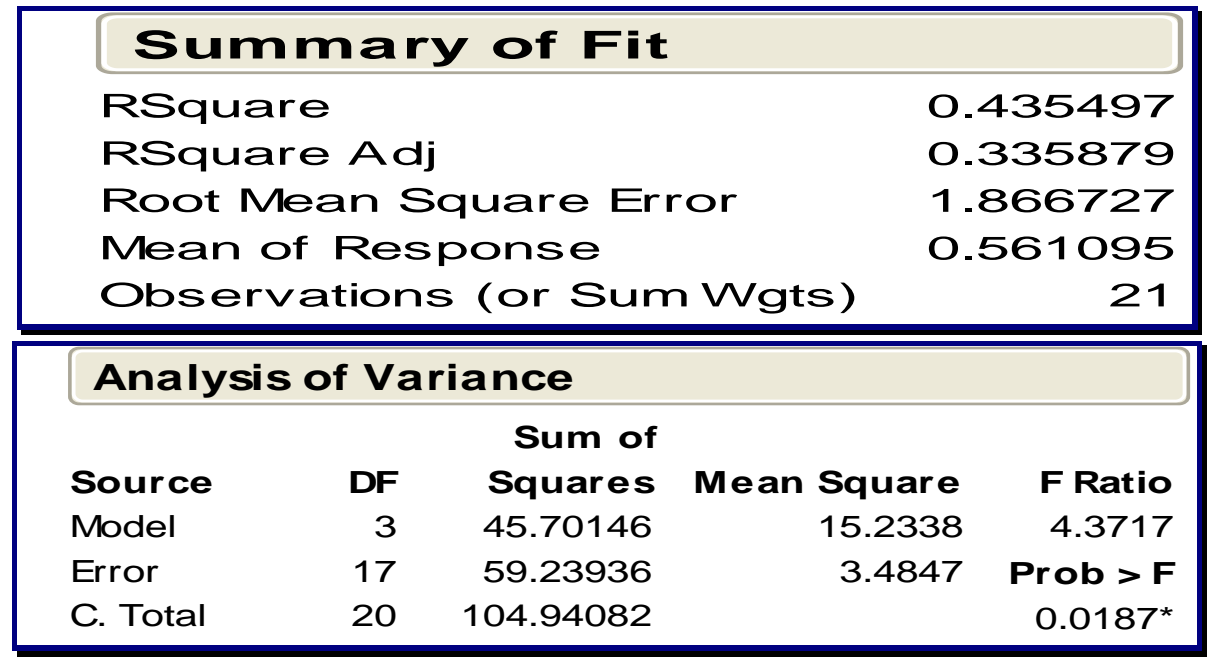

Table aB.6 $\mathrm{Si}_{3} \mathbf{N}_{4}$ etch rate parameter estimates, effect tests, and sorted parameters for the fitted model.

\begin{tabular}{|c|c|c|c|c|c|}
\hline \multicolumn{6}{|l|}{ Parameter Estimates } \\
\hline Term & & Estimate & Std Error & t Ratio & Prob $>|t|$ \\
\hline Intercept & Biased & 3.4532177 & 1.446839 & 2.39 & $0.0289^{*}$ \\
\hline $49 \%$ HF Volume (ml) & & 0.7465918 & 1.183093 & 0.63 & 0.5364 \\
\hline MTES Volume (ml) & & -0.993391 & 0.2847 & -3.49 & $0.0028^{*}$ \\
\hline $\mathrm{H} 2 \mathrm{O}$ Volume (ml) & Zeroed & 0 & 0 & & \\
\hline (49\% HF Volume (ml)-0.85714)*(MTES Volume (ml)-3.47619) & & -1.158957 & 0.546387 & -2.12 & $0.0489^{*}$ \\
\hline$(49 \% \text { HF Volume }(\mathrm{ml})-0.85714)^{*}(\mathrm{H} 2 \mathrm{O}$ Volume $(\mathrm{ml})-200)$ & Zeroed & 0 & 0 & . & . \\
\hline (MTES Volume $(\mathrm{ml})-3.47619)^{*}(\mathrm{H} 2 \mathrm{O}$ Volume $(\mathrm{ml})-200)$ & Zeroed & 0 & 0 & . & . \\
\hline$(49 \% \text { HF Volume }(\mathrm{ml})-0.85714)^{*}(\text { MTES Volume }(\mathrm{ml})-3.47619)^{*}(\mathrm{H} 2 \mathrm{O}$ Volume $(\mathrm{ml})-200)$ & Zeroed & 0 & 0 & & \\
\hline
\end{tabular}

\begin{tabular}{|c|c|c|c|c|c|c|}
\hline Effect Tests & & & & & & \\
\hline Source & Nparm & DF & $\begin{array}{l}\text { Sum of } \\
\text { Squares }\end{array}$ & F Ratio & Prob $>$ F & \\
\hline $49 \%$ HF Volume (ml) & 1 & 1 & 1.387683 & 0.3982 & 0.5364 & \\
\hline MTES Volume (ml) & 1 & 1 & 42.425613 & 12.1749 & $0.0028^{*}$ & \\
\hline $\mathrm{H} 2 \mathrm{O}$ Volume $(\mathrm{ml})$ & 1 & 0 & 0.000000 & . & . & LostDFs \\
\hline $49 \%$ HF Volume $(\mathrm{ml})^{*}$ MTES Volume $(\mathrm{ml})$ & 1 & 1 & 15.678172 & 4.4992 & $0.0489^{*}$ & \\
\hline $49 \% \mathrm{HF}$ Volume $(\mathrm{ml})^{*} \mathrm{H} 2 \mathrm{O}$ Volume $(\mathrm{ml})$ & 1 & 0 & 0.000000 & . & . & LostDFs \\
\hline MTES Volume $(\mathrm{ml})^{*} \mathrm{H} 2 \mathrm{O}$ Volume $(\mathrm{ml})$ & 1 & 0 & 0.000000 & . & . & LostDFs \\
\hline $49 \%$ HF Volume $(\mathrm{ml})^{*} \mathrm{MTES}$ Volume $(\mathrm{ml})^{*} \mathrm{H} 2 \mathrm{O}$ Volume $(\mathrm{ml})$ & 1 & 0 & 0.000000 & . & . & LostDFs \\
\hline
\end{tabular}

\begin{tabular}{|c|c|c|c|c|}
\hline \multicolumn{5}{|l|}{ Sorted Parameter Estimates } \\
\hline Term & & Estimate & Std Error t Ratio & Prob $>|t|$ \\
\hline MTES Volume (ml) & & -0.993391 & $0.2847 \quad-3.49$ & $0.0028^{*}$ \\
\hline (49\% HF Volume (ml)-0.85714)*(MTES Volume (m) -3.47619$)$ & & -1.158957 & 0.546387 & $0.0489^{*}$ \\
\hline $49 \%$ HF Volume (ml) & & 0.7465918 & 1.183093 & 0.5364 \\
\hline H2O Volume (ml) & Zeroed & 0 & 0 & \\
\hline (49\% HF Volume (ml)-0.85714)*(H2O Volume (ml)-200) & Zeroed & 0 & 0 & \\
\hline (MTES Volume (ml)-3.47619)*(H2O Volume (ml)-200) & Zeroed & 0 & 0 & . \\
\hline$(49 \% \text { HF Volume }(\mathrm{ml})-0.85714)^{*}(\mathrm{MTES} \text { Volume }(\mathrm{ml})-3.47619)^{*}(\mathrm{H} 2 \mathrm{O}$ Volume $(\mathrm{mll})-200)$ & Zeroed & 0 & 0 & \\
\hline
\end{tabular}




\section{Appendix C}

\section{List of Publications, Awards, and Patents/Disclosures}

1. Nabil G. Mistkawi ${ }^{1,2}$, Makarem A. Hussein ${ }^{2}$, Malgorzata Ziomek-Moroz ${ }^{3}$, Shankar

B. Rananavare "A Study of Copper Thin Film Dissolution Kinetics in Organic HF Containing Cleaning Solution” J. Electrochem. Soc. Submitted (2010)

2. Nabil G. Mistkawi ${ }^{1,2}$, Makarem A. Hussein ${ }^{2}$, Malgorzata Ziomek-Moroz ${ }^{3}$, Shankar

B. Rananavare " Corrosion Behavior of Copper Thin Films in Organic HF Containing Cleaning Solution for Semiconductor Applications" J. Electrochem. Soc. 157, C24 (2010).

3. The Secret to Developing Wet Etch Chemistry for enabling sub $50 \mathrm{~nm}$ Process Technology Nodes. (Manuscript in preparation)

4. A statistical approach to the Design of Wet Etch Clean Chemical Formulations. (Manuscript in preparation).

\section{Awards}

1. Chemistry Department (Portland State University) Achievement Award for Academic Excellence (Paul Emmett award). (2009)

2. Nickel/Platinum Alloy selective wet etch/clean formulation development and module design/set-up award (Logic Technology Development (LTD), Intel Corporation). (2009)

3. Q1 Materials DRA, for efforts in backend cleans (1266) development partnership with GFM for Backend cleans cost savings of $\$ 30 \mathrm{M}$; enabled an OGA technical solution for P1266.

4. Selective Titanium Wet Etch Chemical Formulation development award (LTD, Intel Corporation). (2006)

5. Golden Global Environmental award for the invention of a green chemical formulation used at Intel (Intel Corporation) (2006). 
6. 1997 PTD Design of Experiment award, for the development of Ni selective etch process (1997).

\section{Patents/Disclosures}

1. A Method for Stabilizing $\mathrm{pH}$ and Improving Clean Ability during Selective Removal of Un-reacted Ni-Pt/Ni Post Salicide Formation while Maintaining High Compatibility to Metal Gate Components. (Intel Disclosure \# 72416) (2009).

2. Selective Nickel Etch Process with High Compatibility to Metal Gate Components (Intel Disclosure \# 61295). (2008).

3. Mega Sonic Assisted Cleans to Selectively Remove Un-Reacted Ni-Pt/Ni Post Salicide Formation with High Compatibility to Metal Gate Components (Intel Disclosure \# 61294). (2008).

4. Composition for etching a metal hard mask material in semiconductor processing. Patent Pending, (2006).

5. Si containing precursors as a source of silicic acid to suppress silicon oxide etch rate in phosphoric acid (Silicon Nitride selective etch). Patent pending (2005).

6. Process to remove carbon doped oxide (CDO) thin films from silicon wafers. (Intel Disclosure 43765). (2005).

7. Process to remove silicon carbide ( $\mathrm{SiC}$ ) thin films from Si wafers. (Intel Disclosure 40696). (2004).

8. Addition of metal corrosion inhibitor Benzotriazole (BTA) to a semi-aqueous corrosive solvent, such as AP-761, as a method to improve its selectivity to metals and alloys. (Intel Disclosure 40193). (2004). 